\title{
Mechanisms underlying the functions of sleep in aging during starvation in \\ Caenorhabditis elegans
}

\author{
Dissertation \\ for the award of the degree \\ "Doctor rerum naturalium" \\ (Dr. rer. nat.) \\ of the Georg-August-Universität Göttingen \\ within the doctoral program Systems Neuroscience \\ of the Göttingen Graduate School for Neurosciences, Biophysics, and \\ Molecular Biosciences (GGNB) \\ submitted by \\ Yin $\mathrm{Wu}$ \\ from Shaanxi, China
}

Göttingen 2019 



\title{
Thesis Committee Members
}

Prof. Dr. Henrik Bringmann

( $1^{\text {st }}$ Reviewer)

Prof. Dr. Ralf Heinrich

( $2^{\text {nd }}$ Reviewer)

Prof. Dr. Nils Brose
Max Planck Research Group "Sleep and Waking"

Max-Planck-Institute for Biophysical Chemistry, Göttingen

Department of Cellular Neurobiology

Schwann-Schleiden Research Center, Göttingen

Department of Molecular Neurobiology

Max-Planck-Institute for Experimental Medicine, Göttingen

\section{Examination Board Members}

\author{
Arezoo Pooresmaeili, M.D.,Ph.D Research Group Perception and Cognition \\ European Neuroscience Institute, Göttingen
}

Prof. Dr. Andreas Stumpner

Prof. Dr. Reinhard Schuh

Date of oral examination: $28^{\text {th }}$ June 2019

\author{
Department of Cellular Neurobiology \\ Schwann-Schleiden Research Center, Göttingen
}

Research Group "Molecular Organogenesis"

Max-Planck-Institute for Biophysical Chemistry, Göttingen 



\section{Abstract}

Sleep is vital for living organisms. Disruption of sleep is a hallmark of natural aging. A growing body of evidence suggests that sleep serves roles in nervous system-specific functions as well as general cell physiological functions related to aging. However, the molecular mechanisms underlying the connection between sleep and aging are still poorly understood.

In this study, I have focused on starvation-induced sleep in larval C. elegans, and aimed to figure out the sleep functions and the mechanism that connect sleep, starvation, survival, and aging. First, I found that the sleep-promoting neuron RIS is activated by starvation through sirtuin signaling and PTEN by further activating the longevity regulators AMP kinase and FoxO, respectively. Next, I discovered that the potential functions of sleep are involved in several fundamental physiological mechanisms such as proteostasis, apoptotic cell death, and autophagy as well as the aging process in starved larval C. elegans. Lastly, I identified a novel protective anti-aging mechanism of sleep in arrested L1 larval worms, which interacts with the mitochondrial respiratory chain, TOR signaling and the unfolded protein response in regulating survival and aging during food deprivation.

The discoveries in this study suggest that sleep is required to ensure the survival by counteracting aging processes of larval $C$. elegans during prolonged starvation, and sleep acts as a predictor for the longevity of the starvationinduced developmental arrest. As a fundamental process, sleep presents a beneficial strategy to protect living organisms against aging and starvation in the larval period. These findings indicate that the functions of sleep have been selected for early in evolution and might be conserved in humans. 



\section{Table of contents}

1. Introduction....................................................................................................................................

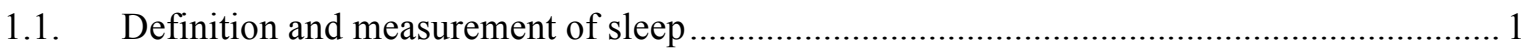

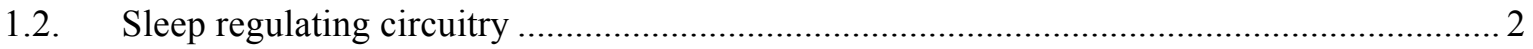

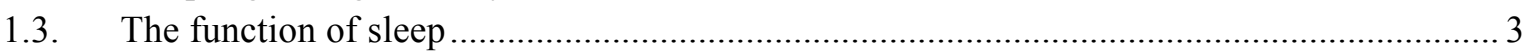

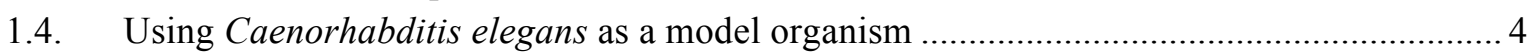

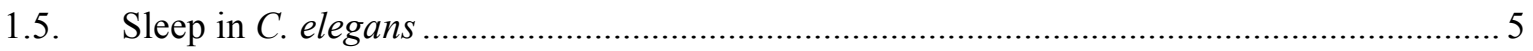

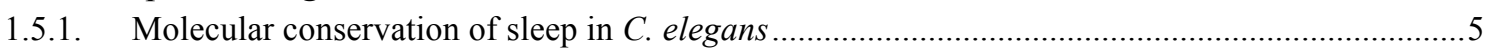

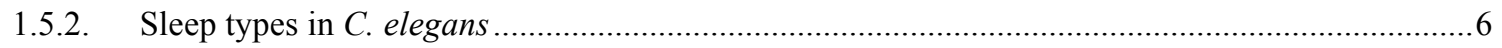

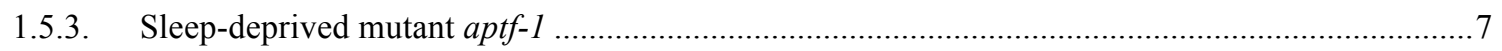

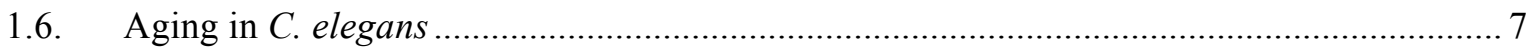

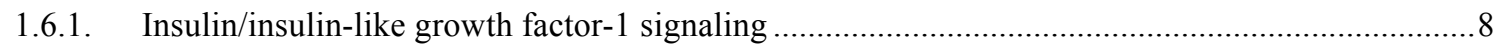

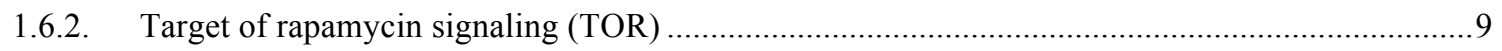

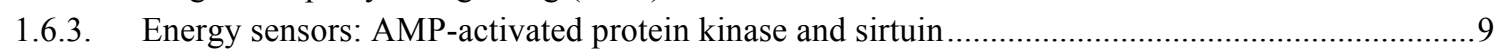

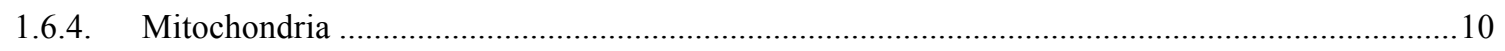

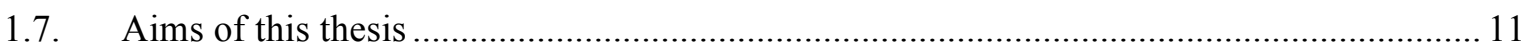

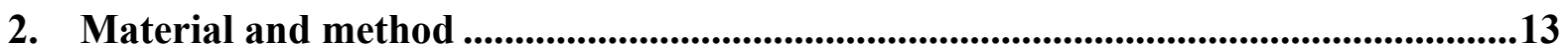

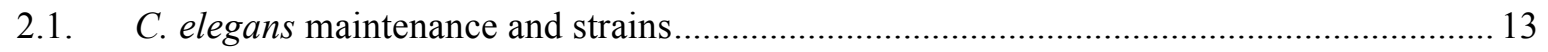

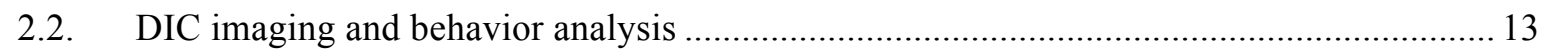

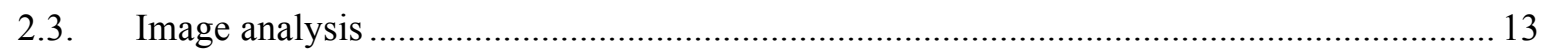

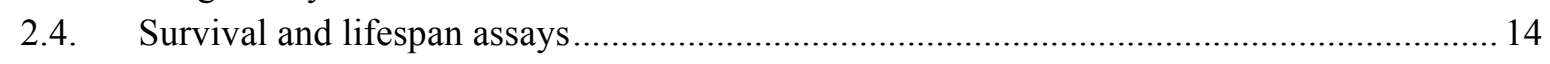

2.4.1. Survival span of arrested L1 larvae in M9 buffer and on NGM plate........................................14

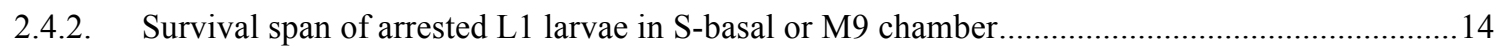

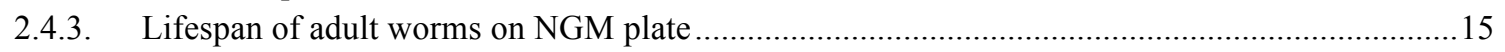

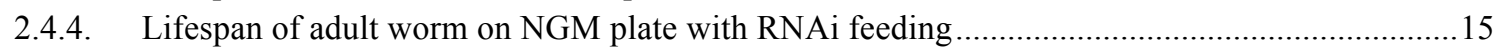

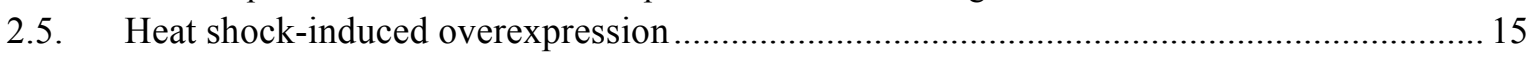

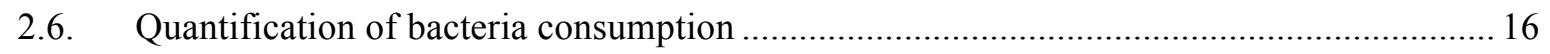

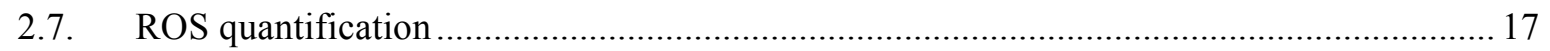

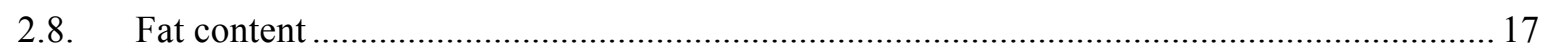

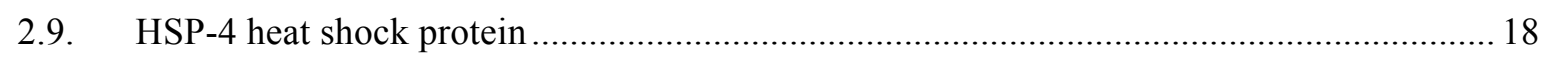

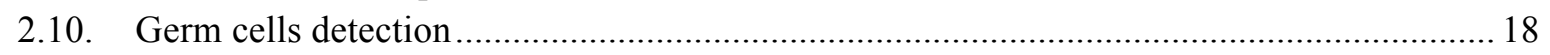

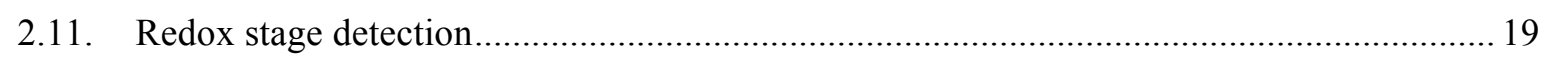

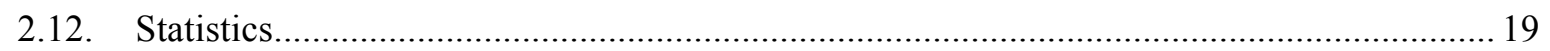

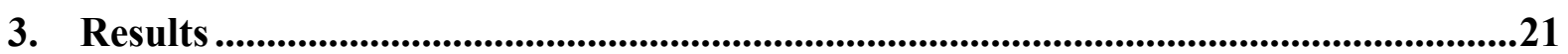

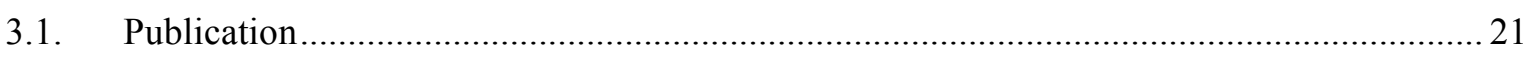

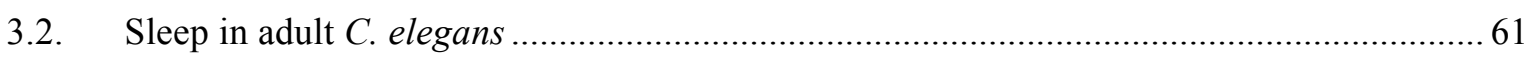

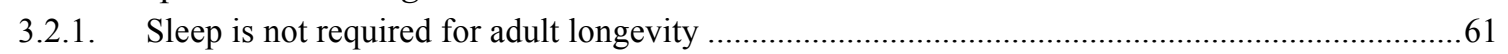

3.2.2. Sleep does not affect adult lifespan expectancy caused by a metabolic alteration..........................61

3.2.3. Sleep is dispensable for TOR and FoxO signaling pathway for regulating lifespan of adults......62

3.3. Sleep plays a central role in starvation-survival of arrested L1 C. elegans ............................... 64

3.4. Metabolic alteration is involved in the sleep-protective program during starvation............. 64

3.5. The decrease of survival in the sleepless mutant is recovered by ethanol .............................66

3.6. Over-expression of FLP-11 does not benefit survival in arrested L1 larvae.......................... 69

3.7. AMPK and PTEN are further involved in the protective program of sleep......................... 70

3.8. Sleep is related to RNA metabolic process in L1 larvae during starvation.......................... 72 
3.9. TOR signaling is involved in the protective sleep program during starvation.....................73

3.10. Apoptotic cell death and autophagy are involved in the protective program of sleep ......... 75

3.11. Mitochondrial respiratory chain is involved in the protective program of sleep ................. 76

3.12. Sleep might contain two independent mechanisms for adult worms and L1 larvae ............78

3.13. Sleep deprivation reduces the ROS generation in starved L1 larvae ................................. 79

3.14. Sleep increases the redox stage throughout of survival in arrested L1 larvae...............81

3.15. Sleep did not affect the cell-cycle quiescence in L1 larvae during starvation ....................... 83

3.16. Sleep improves the unfolded protein response in resisting ER stress in L1 larvae............... 85

3.17. Sleep promotes the $h s p-16.2$ expression after heat shock in early-starved L1 larvae.........86

3.18. Energy exhaustion is not the main reason for the premature death of L1 larvae ............... 89

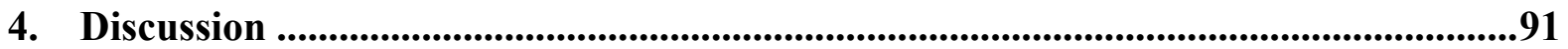

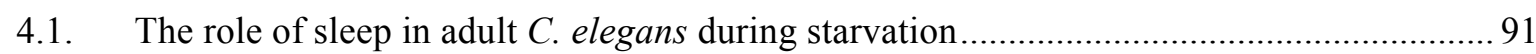

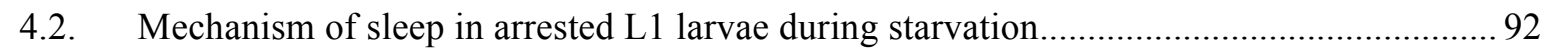

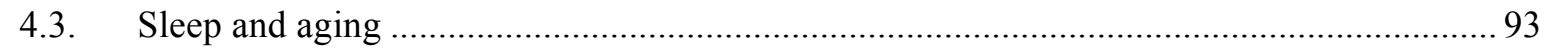

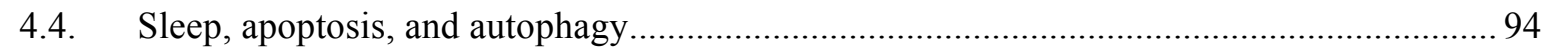

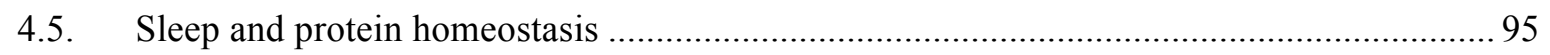

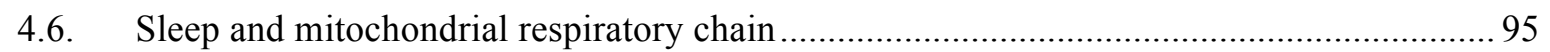

4.7. Sleep might contain two partly distinct mechanisms ......................................................... 97

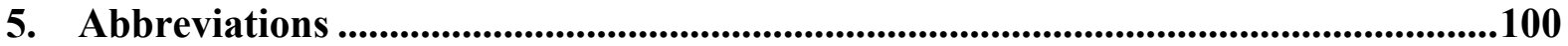

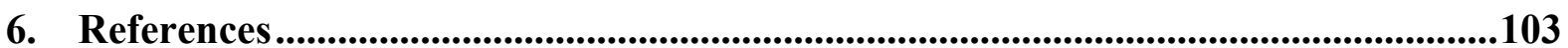

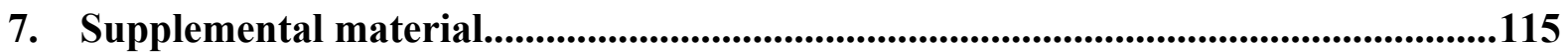

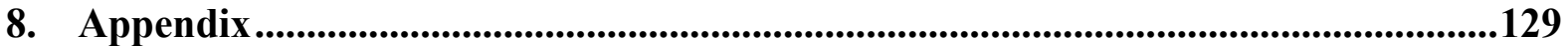

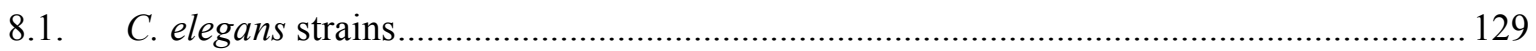

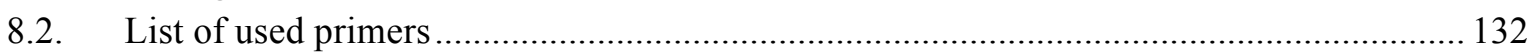

9. Acknowledgments .............................................................................................................135 




\section{Introduction}

\subsection{Definition and measurement of sleep}

Sleep is a fundamental physiological behavior and occurs across the animal world, including mammals, birds, fish, insects, and nematodes Siegel [1]. However, sleep patterns vary widely across species. In mammals, several factors influence the total sleep time like brain size, body mass index, diet as well as the social hierarchy [2, 3]. Certain animals such as little brown bats Myotis lucifugus spend 19.9 hours a day in sleep while African elephants Loxodonta Africana sleep only for 2 to 3 hours a day [4, 5].

The definition of sleep is based on five key criteria: 1) Sleeping animals show behavioral quiescence with species-specific posture. 2) During sleep, animals have decreased brain arousal and reduced responsiveness to stimuli. 3) Sleep is rapidly reversible. 4) Sleep contains a mechanism of homeostatic regulation; i.e., prolonged or deeper rebound sleep occurs as a consequence of sleep deprivation. 5) Sleeping animals show a different electrical brain activity pattern in comparison to the brain pattern of awake animals $[2,6]$.

Sleep in mammals can be measured by electroencephalography (EEG), whereby the synchronous electrical activity of the brain surface is measured by placing electrodes on the scalp [7]. Based on the occurrence of brain waves of different amplitudes (power spectral density of EEG), human sleep can be divided into two main distinct stages: rapid eye movement (REM) and non-REM (NREM) sleep.

During REM sleep, desynchronized brain activity is shown in a low amplitude signal with a high power spectral density in the EEG pattern similar to the waking stage. People show rapid eye movement, physiological muscle atonia and partial loss of homeothermy. The NREM sleep is unlike REM sleep as only a little or no eye movement is observed in this stage. The EEG pattern in NREM shows a highly synchronous brain activity without muscles paralysis [8] and may lead to sleepwalking. Moreover, the deepest sleep stage — slow-wave sleep (SWS) - also occurs in this stage. The time of one complete NREM-REM cycle takes around $1.5 \mathrm{~h}$ on average [9]. 
The polygraphic recording procedure is a gold standard to assess sleep in both mammals and birds in classic sleep research. Despite this, EEG signatures cannot be recognized in invertebrates and are inadequate for determining sleep in all animal species. Hence, only the first four criteria without the detection of electrical brain activity have been applied to identify sleep in non-mammals species.

Based on the four key behavioral criteria of sleep, sleep has been distinguished in the nematode Caenorhabditis elegans [10-12], the common fruit fly Drosophila melanogaster [13, 14], and Zebrafish Danio rerio [15]. Over the past few decades, the studies of sleep in simple model organisms such as these have been greatly contributed to the understanding of the regulatory mechanisms and molecular components underlying sleep and of course, have become a cornerstone of sleep research.

\subsection{Sleep regulating circuitry}

In the mammalian brain, sleep and wakefulness are regulated and balanced by a socalled flip-flop switch mechanism. This mechanism consists of two major elements: the ascending arousal system and the ventrolateral preoptic nucleus (VLPO). They are mutual inhibitors responsible for wakefulness and sleep, respectively. During wakefulness, the components of the ascending arousal system such as the locus coeruleus (LC), the tuberomammillary nucleus (TMN) and the raphe nuclei inhibit the sleep-activating region in the brain and keep the animal awake via several monoaminergic neurotransmitters, including dopamine, noradrenaline and histamine as well as serotonin $[16,17]$. In reverse, during the sleeping time, this "keep awake" ascending arousal system is inhibited by the VLPO directly through the inhibitory neurotransmitters such as $\gamma$-aminobutyric acid (GABA) and galanin (GAL) [18-20]. At the same time, VLPO is also able to inhibit the orexinergic neurons that play a role in the regulation of wakefulness by releasing the neuropeptide orexin [16, 21].

Moreover, previous studies of sleep have reported that the circadian rhythms and homeostatic processes also play interactional roles in sleep regulation [22, 23]. Sleepactive neurons in preoptic area of the brain are further activated by sleep deprivation and an increase in sleep time was observed after the extension of the anterior deprivation 
time during sleep $[17,24]$. However, the complete sleep-regulatory mechanisms are still not understood fully and need further exploration.

\subsection{The function of sleep}

Although sleep is an ancient mechanism, little is known about its origin and evolution [25]. In the wild environment, most animals have to be constantly on the alert for surrounding to avoid predators and forage for food. Sleeping does not seem to be an advantage for survival in the natural selection process. However, sleep was still included in the early stages of evolution across animals and has been conserved ever since. This suggests that at least one even greater selection pressure is involved in maintaining sleep across species.

It has been proposed through several hypothetical theories that sleep might be involved in various physiological mechanisms and be required for diverse life aspects. One of the hypotheses claims that sleep is supposed to play a role in nervous-system-specific functions. Although sleep benefits the entire body of animals, it has more direct and unavoidable impact on the brain functions [26]. Analyses of the sleeping brain have suggested that sleep contributes to the synaptic plasticity/downscaling, thereby supporting the memory consolidation after learning [27-29]. NREM sleep in mammals has been reported to involve in this functional aspect of memory-formation and REM sleep may further help as an assistant through the rhythmic regulation of the hippocampus [30-32].

Another hypothesis proposes that sleep can contribute to different core molecular and cellular functions, such as energy conservation, body healing, removal of reactive oxidative molecules and macromolecule biosynthesis [33]. Previous studies reported that sleep benefits growth, stress resistance, and immune defense by improving the anabolic metabolism [11, 34-36].

On the whole, most of the theories of sleep's function are established by observing sleep processes and correlating with the consequences of various sleep deprivation experiments. Sleep seems to serve multiple functions in many aspects depending on the 
species. Still, we have been able neither to identify the core universal function of sleep, nor to clearly explain why animals need this state of quiescence in their life.

\subsection{Using Caenorhabditis elegans as a model organism}

Since Sydney Brenner introduced Caenorhabditis elegans into biological research 50 years ago, this $1 \mathrm{~mm}$ long nematode became one of the most excellent model organisms for investigating molecular and neurobiology [37]. In the laboratory, most of the $C$. elegans are self-fertilizing hermaphrodites, only a few are males with a frequency of $<0.2 \%$ [38]. At $20^{\circ} \mathrm{C}$, a wild-type hermaphrodite (N2) can live around $2-3$ weeks in the presence of food and lay approximately 300 eggs.

C. elegans has a very short life cycle. The embryogenesis occurs in the egg and is retained in utero within the hermaphrodite until 24-cell stage. After egg-laying, the embryo hatches and becomes the L1 larva and starts to eat. The whole larval development process takes around 2.5 days depending on the temperature of the undergoing four stages form L1 to L4. At the end of each stage, a sleep-like period (namely lethargus) occurs for 1.5 to 2 hours [10]. After sleep, the worm starts to molt and enters the next stage.

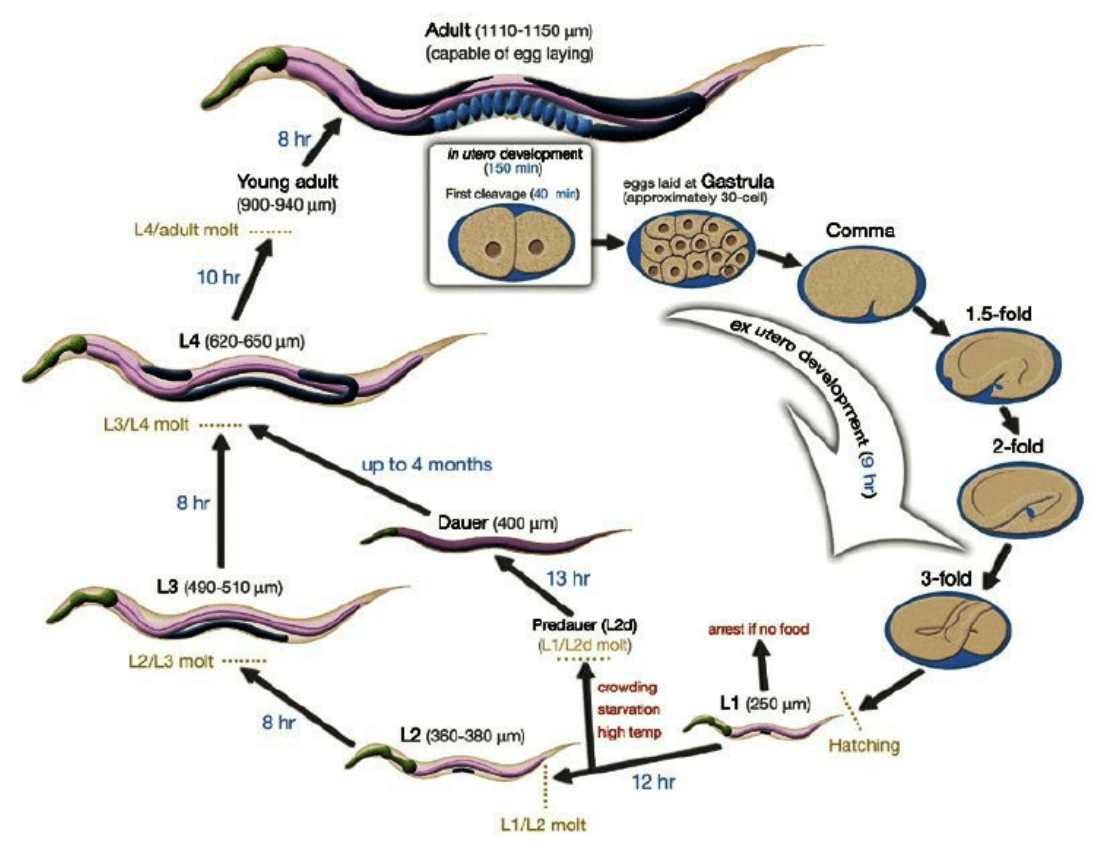

Figure Introduction 1 C. elegans developmental cycle. Figure shows the development process with the time required of $C$. elegans from egg to adult hermaphrodite at $22^{\circ} \mathrm{C}$. The figure is taken from WormAtlas.org (Introduction to C. elegans anatomy) 
Under some unpleasant environmental conditions, such as high population density, food insufficiency or stressful temperature, the larva evolves into an alternative "dauer" stage after L1 [39]. The dauer larva has a special cuticle with high stress resistance and can survive for several months in starvation. However, even when the food is not enough to support the larva to reach the dauer stage, the larva is able to arrest in the L1 stage after hatching, which is called as "arrested L1". Unlike the dauer stage, arrested L1 does not change the morphology of the cuticle and can survive for only several weeks in starvation [40]. After feeding, both dauer and L1-arrested larva can re-enter the developmental cycle.

C. elegans is the first multicellular organism that has a fully-sequenced genome. More than 7000 protein-coding genes of $C$. elegans were identified as homologous to human genes [41]. Furthermore, the whole cell lineage and the complete connectome of $C$. elegans have been mapped in previous researches. An adult hermaphrodite contains 956 somatic cells and 302 neurons, and an adult male carries 1031 somatic cells and 383 neurons in the body $[42,43]$. Therefore, C. elegans serves as a fundamental model system for studying the combination of behavior, molecular genetics and neuronal circuits.

\subsection{Sleep in C. elegans}

\subsubsection{Molecular conservation of sleep in C. elegans}

Quiescence states that satisfy all the behavioral criteria of sleep have been identified in $C$. elegans. Although the worm sleep does not follow the well-known $24 \mathrm{~h}$ pattern, it is still regulated by the transcription factor LIN-42 that is a homolog of the protein PERIOD and expresses circularly with the circadian timer in mammals and Drosophila [44-46]. Furthermore, five conserved regulatory signaling pathways for sleep have been found between Drosophila, mammals and C. elegans: pigment dispersing factor (PDF), proteinkinase A/Cyclic adenosine 3', 5'-monophosphate (PKA/cAMP), epidermal growth factor receptor (EGFR), dopamine and protein kinase $G$ (PKG) signaling pathways [10, 11, 47-51]. 


\subsubsection{Sleep types in C. elegans}

In C. elegans, two major states of sleep were well characterized. One is called stressinduced sleep (SIS). This state occurs when the worm suffers cellular stresses under different stimulus conditions. The duration is different, depending on the stressor [11, 52]. SIS is not only shown in the adult worm but also occurs in larva all through the developmental state. The single sensor neuron ALA is required for stress-induced sleep. This interneuron can be activated via epidermal growth factor (EGF) signaling and induces sleep through the endocrine mechanism by releasing neuropeptides such as FLP$13[11,48,53]$. However, we still do not know about the homeostatic response of the SIS deprivation. Whether the depolarization of ALA is specifically responsible for sleep onset has not been reported yet. Impairment of stress-induced sleep was observed in the ALA ablated worms [48].

The other well-known sleep state is called lethargus, also known as developmentally timed sleep (DTS) in some studies. This sleep occurs for approximately 2 hours before each of the four molts during the larval development. Neuronal activity is reduced widely during lethargus, and it is homeostatic regulated [10, 54-56].

Two interneurons have been identified as regulatory neurons in lethargus: the paired glutamatergic neuron RIA and the GABAergic neuron RIS [12, 53]. RIA can inhibit the locomotion and feeding activity during lethargus by releasing the neuropeptide NPL-22. The expression of NPL-22 is regulated by the gene lin-42. However, the activation of RIA seems only to play a role for sleep in larva, but not in adult worms [53, 57]. RIS is the second regulatory neuron that depolarizes at the onset of sleep as sleep-promoting and sleep-active neuron. It plays a crucial role in lethargus. The ablation of RIS shows a complete elimination of quiescence during lethargus.

Interestingly, RIS activation can be suppressed through a waking stimulus. It indicated that $C$. elegans has a similar mechanism to the flip-flop switch of mammals in regulating sleep $[12,58]$. Moreover, John White carried out a number of synaptic associations in both upstream and downstream of RIS. Some neurons were reported to be interrelated with RIS via chemical synapse [43]. However, we still do not have sufficient data for 
revealing the complete innervated network and regulatory circuits of ALA and RIS for sleep. Both neurons are essential to sleep but appear to work in parallel systems.

Beside the two major sleep states, satiety quiescence was also observed following a high-quality food feeding after starvation in C. elegans. The ASI neuron plays a role in regulating satiety quiescence via the transforming growth factor $\beta$ (TGF $\beta)[59,60]$.

\subsubsection{Sleep-deprived mutant aptf-1}

The AP-2 transcription factor family contains five different proteins. In human, mutations of TFAP2 $\beta$ lead to the Char syndrome, which is related to insomnia [61]. Moreover, the AP-2 homolog TfAP-2 in Drosophila is necessary for sleep in the night [62]. In C. elegans, the homolog of AP2 transcription factor is APTF-1, which is expressed in five head neurons including the paired interneurons of AIB and RIB as well as the sleep-active and sleep-promoting neuron RIS. The previous study of Turek demonstrated that APTF-1 is required in RIS for inducing sleep through the release of the inhibitory RMRFamide-like neuropeptide FLP-11 [58]. Deletion of aptf-1 leads to a complete deficiency of quiescence during lethargus in C. elegans [12]. However, beyond that, no apparent phenotypes such as lifespan deficiency, locomotion variant or development abnormality have been observed in the adult aptf-1 mutant in comparison to the wild-type worms. Interestingly, the consequence of a lack of sleep seems not to occur in adults. Thus the primary function of C. elegans's sleep in the early stage of evolution becomes very interesting and worthy of the research of sleep.

\subsection{Aging in C. elegans}

In recent decades, aging has become one of the most major subjects in the fundamental research in biology. C. elegans serves as a well-established model tool and has contributed several key discoveries for our understanding of the aging process. Since the identification of the first lifespan-regulatory pathway (the insulin/insulin-like growth factor-1 signaling (IIS) [63]), an increasing number of genetic factors have been found to correlate the aging process. Environmental pressures have been identified as major factors in the aging progress and are associated with stress-resistance and longevity. 
What we recently know about the aging-regulatory signaling in C. elegans is majorly focusing on the nutrient-sensing pathways including the IIS signaling, the target of rapamycin signaling (TOR), the sirtuin signaling and the AMP-activated protein kinase signaling (AMPK). Besides, other factors such as mitochondria, proteostasis and hypoxia-inducible factor 1 as well as the epigenetic mechanisms have also been shown to interact with the aging process significantly.

\subsubsection{Insulin/insulin-like growth factor-1 signaling}

As the major nutrient-sensing pathway in aging, IIS signaling requires three key components: DAF-2 (homolog of the insulin/insulin-like growth factor-1 (IGF-1) receptor) [64, 65], AGE-1 (homolog of phosphatidylinositol 3-kinase) [66, 67] and DAF-16 (homolog of the forkhead box (FoxO) transcription factor) [68, 69]. The nutritional restriction decreases the activity of DAF-2 and subsequently decreases the phosphoinositide (PI) 3-kinase signaling via AGE-1/PI3K. Contiguously, it activates and promotes the nuclear translocation of the transcription factor DAF-16 and thus further activates/represses numerous DAF-16 target genes that relate to metabolism, autophagy, and proteostasis in the cellular response as well as the stress response including heatshock, oxidative stress and detoxification [70-73]. Ultimately, the prolonged lifespan with the increased adaptability to stresses allows the worm to survival in the unfavorable environments and obtain more chances to maintain the next generation.

Furthermore, several additional factors have been reported to participate in the IIS pathway and assist DAF-16 to active the appropriate genes according to the context. For example, the tumor suppressor DAF-18 down-regulates the IIS signaling by hydrolyzing PIP3, which is produced in the downstream of AGE-1/PI3K [74]. The JNK-1 (c-Jun Nterminal kinase subgroup) and the CST-1 (ortholog of the mammalian Ste20-like kinases) promote the DAF-16 activity through the post-translational modification $[75,76]$. The type I protein arginine methyltransferase PRMT-1 and the E3 ubiquitin ligase RLE-1 are also able to influence DAF-16 in nuclear maintenance and molecular stability respectively $[77,78]$. 


\subsubsection{Target of rapamycin signaling (TOR)}

TOR signaling act together with the IIS signaling in the regulatory mechanisms of longevity and it is majorly regulated by the nutrient consumption in C. elegans.

In mammalian cells, TOR kinases consist of two functionally distinct complexes: TORC1 and TORC2 [79]. This signaling pathway impacts many important physiological mechanisms such as development, lipid storage, protein synthesis, transcription/translation and autophagy as well as the longevity in C. elegans [80-82]. The extension of lifespan by down-regulating the TOR signaling requires DAF-16 and is mediated by several additional factors including HSF-1 (ortholog of heat-shock transcription factor), HIF-1 (ortholog of hypoxia-induced factor), PHA-4 (FoxA transcription factor) and SKN-1 (ortholog of nuclear factor-erythroid-related factor) [8390].

\subsubsection{Energy sensors: AMP-activated protein kinase and sirtuin}

As an energy sensor, AMPK is activated by the rise of ADP: ATP and/or AMP: ATP ratios. AMPK can stimulate ATP production and decrease energy consumption, thus promoting cellular survival in the low energy condition [91].

In C. elegans, AMPK and DAF-16 interact together by a signaling feedback loop in response to calorie restriction. Lack of AAK-2, which is an AMPK $\alpha$ subunit, shortens lifespan. Overexpressing the AAK-2 prolongs the worm longevity. The underlying mechanism is mediated by DAF-16. AAK-2 is also required for the IIS-related longevity for the DAF-16 activation as well [92-94]. Another energy sensor is a group of proteins called sirtuins. They are part of the family nicotinamide adenine dinucleotide (NAD)dependent protein deacetylases. Sirtuins react to a high $\mathrm{NAD}^{+}$level caused by low energy state in cell, and also affect the processes associated with aging, apoptosis and stress-responses [95].

In C. elegans, the homologous protein of sirtuin is SIR-2.1. Increasing the SIR-2.1 expression level leads to an extension of lifespan in adults. The longevity effect shows a dependency of DAF-16 and AAK-2 [96-98]. Knocking out the gene sir-2.1 leads to an 
elimination of longevity effects caused by the other factors. This study further supposes the positive role of SIR-2.1 in the lifespan prolongation from another aspect.

Both AAK-2 and SIR-2.1 induced longevity effects are similar to the effect caused by the eat-2 gene knockout that mimics the chronic energy restriction [81, 99]. However, the longevity effect of AAK-2 is not present in the middle-aged animals. The AMPK signaling seems unnecessary for the lifespan prolongation caused by energy restriction $[81,100,101]$. It suggested that the effect of sirtuin and AMPK that regulate longevity in worms is under some certain conditional restrictions.

\subsubsection{Mitochondria}

Since the reactive oxygen species (ROS) has been identified as a key role in the aging processes [102], an increasing number of aging studies focused on the mitochondrial respiratory chain (MRC) and energy generation. The results of the large-scale RNAi screen have demonstrated that most of the longevity-related genes are associated with metabolic mechanisms and half of those genes code for the components of the mitochondrial electron transport chain (ETC) [103-106].

Moreover, some mitochondria-related factors not involved in the MRC have been found to relate longevity in mitochondrial unfolded protein response, translation and autophagy processes [107, 108]. In an aged worm, mitochondrial efficiency is relevant to the mitochondrial antioxidant capacity [109]. However, many studies have argued that oxidative stress cannot be simply represented by ROS production and clearance [110, 111]. For instance, the sod-2 loss-of-function mutant that is lacking in the mitochondrial superoxide dismutase SOD-2 shows not only a rise of oxidative damage with high level of ROS, but also an increased lifespan [112]. Furthermore, the extension of lifespan and a high ROS level both occur after the respiration inhibition. It has been reported that the AAK-2, CEP-1 and SKN-1 play roles in this effect [113-115]. Moreover, loss of movement caused by the age-related decline in muscle has been shown to be associated with the destruction of functional mechanisms in mitochondria [116]. 


\subsection{Aims of this thesis}

C. elegans serves as an ideal tool for studying sleep mechanisms in biology. The worm sleeps not only during larval stages before molting but also during the arrested diapause as well as under different stress conditions $[10,11,117]$. Recent research has step-bystep identified the sleep-related neurons and their neural networks in C. elegans. However, the core function of sleep and its underlying molecular mechanisms are still wrapped in mystery.

The aptf-1 mutant, which lacks the APTF-1 transcription factor in the sleep-active neuron RIS, shows a complete quiescence deficiency during sleep [12]. Therefore, it is an excellent model for studying sleep. Interestingly, a significant decrease in survival was found in arrested aptf-1 larvae during starvation. These observations suggest that quiescence may play an essential role in survival in the case of food deprivation. The underlying mechanisms are assumed to be connected with reversible aging in the arrested L1 larvae, which displays several age-related phenotypes similar to those found in the aged adult $C$. elegans [117].

In this study, I aimed to figure out the molecular connections between sleep, aging and starvation and the underlying mechanisms in C. elegans. To achieve this goal, I first measured the survival rate and connected it with the total sleep time of single arrested L1 larva and thus determined the first link between sleep and survival of the L1 larvae during starvation. I then tested several age-related biomarkers throughout the survival span such as morphology of mitochondria; polyglutamine aggregation; ROS/Redox stage, and the proteostasis in response to stress in the sleepless aptf-1 mutant L1 larvae during starvation. The results have strongly supported that sleep has a very tight connection with aging in larval C. elegans.

Next, I aimed to identify the molecular mechanisms of: How starvation actives sleep behavior (upstream signaling pathway of RIS)? And how the activated sleep behavior further influences aging (downstream signaling pathway of RIS)? To pursue this aim, I did a mutation screen of sleep bouts and as well as of survival time during L1 starvation. Several related biological mechanisms that relate to the sleep-active/sleep-promoting 
pathway and the protective program of sleep were identified in this study. A part of my results has been published in Current Biology [118].

My research gives an initial insight into the connection of sleep and aging in C. elegans. Furthermore, an aging-related modulatory mechanism of sleep was determined in the arrested L1 larval worms under starvation. This study will therefore help us further understand the vital role of sleep during evolution and its biological functions in living organisms. 


\section{Material and method}

\subsection{C. elegans maintenance and strains}

C. elegans was maintained on nematode growth medium (NGM) plates seeded with bacteria E. coli OP50 as described above [37] at 15,20 or $25^{\circ} \mathrm{C}$. The worm strains used for this study and the sequencing primers that used for the PCR verification of mutations are shown in the appendix.

\subsection{DIC imaging and behavior analysis}

The agarose microchamber imaging (AMI) was used for imaging the sleep/wake behavior of worms $[119,120]$. To obtain the sleep behavior of arrested L1 larvae, I picked eggs were picked to an unseeded NGM plate and then transferred to the $190 \mu \mathrm{m} \mathrm{x}$ $190 \mu \mathrm{m} \times 15 \mu \mathrm{m}$ (X length $\times 1 \mathrm{Y}$ length $\times \mathrm{Z}$ depth) agarose microchamber without bacteria. Starved L1 larvae were placed at $20^{\circ} \mathrm{C}$ incubator for around $24 \mathrm{~h}$ after fresh hatching. Images (contains up to 4 worms/image field) were obtained with the $20 \mathrm{x}$ objective and an additional 0.75 lens. Frame rate was set to 0.1 frames/second unless otherwise noted.

\subsection{Image analysis}

To analyze the velocity of the worm locomotion, two different methods were used with a home-made MATLAB routine according to the requirement of the experiment. For the sleep bout screen assay, the centroid of the entire body of the arrested L1 worms was determined automatically by MATLAB and calculated with a conversion factor into velocity in $\mu \mathrm{m} / \mathrm{s}$. The velocity or frame-subtraction data were subsequently calculated for scoring sleep bouts. The data was firstly smoothed by an in-build smooth function of MATLAB with a first-degree polynomial local regression model for over 20 time points. Parameters were set to a velocity below $0.5 \mu \mathrm{m} / \mathrm{s}$ for a minimum of 3 minutes to be detected as sleep in L1 arrest worms. For the rescue assay of daf-18 arrested L1 mutant, worm locomotion was detected by using a frame-subtraction function of MATLAB. The data was smoothed for 5 time points. The intensity of locomotion that was below $50 \%$ of 
average intensity for a minimum of 3 minutes was scored as sleep. All the cutoff parameters for sleep scoring were determined empirically [121].

\subsection{Survival and lifespan assays}

\subsubsection{Survival span of arrested L1 larvae in M9 buffer and on NGM plate}

To synchronize L1 larvae, eggs were obtained by bleaching of a mixed population of adults and then incubated in the standard M9 buffer in a $2 \mathrm{ml}$ tube on a slowly spinning rotator overnight [122]. Samples with at least 50 to 80 arrested worms were taken out of the tube and transferred to a fresh NGM plate with food for every 1 to 3 days. The number of the living worms was scored 5 to $20 \mathrm{~min}$ after the transference. The mobility of worms was used to distinguish between survival and death. To measure the ability of worm re-entering the development, we kept the seeded NGM plates with the surviving worms until they were able to develop to the late-L4 stage. The 1-day of late-L4 stage was scored for successful development. For the survival span on NGM plate, I transferred the 300 synchronized L1larvae from M9 buffer to fresh unseeded NGM plate. The percentage of death was counted every 2 to 3 days until all the L1 larvae died. All the survival span assays were done at $20^{\circ} \mathrm{C}$ incubator in the dark except the heat shock induced FLP-11 overexpression lifespan, that was done at $25^{\circ} \mathrm{C}$ incubator. The detail of the survival spans that were treated with different chemical substances is shown in the survival span tables individually.

\subsubsection{Survival span of arrested L1 larvae in S-basal or M9 chamber}

For comparing the sleep fraction and the survival rate in the starved L1 larvae, fresh eggs were picked and enclosed in S-basal or M9 agarose microchambers without bacteria. The day after picking the eggs into the chamber was considered as the first day of L1 arrest. The worms were scored every 2 to 3 days. Chambers were re-moisturized with $20 \mu \mathrm{L}$ S-basal containing $100 \mu / \mathrm{ML}$ Nystatin (Sigma Aldrich) in order to prevent the dehydration and contamination of fungi. For the normal survival span assays, chambers were placed in the $20^{\circ} \mathrm{C}$ incubator without light. The heat shock induced FLP11 overexpression survival span was done in the $25^{\circ} \mathrm{C}$ incubator. 


\subsubsection{Lifespan of adult worms on NGM plate}

We used a standard lifespan protocol for feeding adults [123]. The plates were seeded with E.coli as food source. For the assays with different chemical compound, $10 \mathrm{mM} 2-$ deoxy-D-glucose, $100 \mathrm{mM}$ D-glucose or $0.1 \mathrm{mM}$ sodium azide were added in NGM plates respectively before seeding. In the starvation assays, a similar protocol was used as the feeding assay. In order to avoid the formation of "bag of worm" in starved adults, L4 worms were first transferred to seeded NGM plates, which contain $50 \mu \mathrm{M} 5$-fluoro-2deoxyuridine (FUdR, Sigma-Aldrich) for two days and then transferred to unseeded NGM plates with the same concentration of FUdR for lifespan assay [123]. The assays intermittent fasting was followed a modified protocol from Uno [124]. In this assay, worms were fed with the living OP50 instead of the kanamycin-killed OP50 that used in Uno's study. All the lifespan assays were done at $20^{\circ} \mathrm{C}$ in the dark. Worms were transferred to fresh NGM plates if any contamination appeared. For data analysis, we excluded the worms that died due to drying out after crawling off the agarose, being killed by picking or having the phenotype "bag of worms".

\subsubsection{Lifespan of adult worm on NGM plate with RNAi feeding}

For RNAi feeding assay, a standard RNAi feeding protocol was used [125]. RNAi sequences were cloned respectively into the LH440 vector that contains the T7 promoter. HT115 E. coli strain was then used to express the LH440 vector because this strain is able to express T7 RNA polymerase after induction by isopropyl- $\beta$-dthiogalactopyranoside (IPTG) [126]. HT115 was incubated at $37^{\circ} \mathrm{C}$ overnight and then seeded to fresh NMG plates containing $1 \mathrm{mM}$ IPTG (Sigma Aldrich) and $25 \mu \mathrm{g} / \mathrm{ml}$ carbenicillin (carbenicillin disodium salt, Biomol). Afterwards, I transferred the L4 worms to the plates and followed the standard lifespan protocol for feeding adults as described above. The whole lifespan assays were done at $20^{\circ} \mathrm{C}$ in the dark. Worms were transferred to fresh NGM plates if any contamination appeared.

\subsection{Heat shock-induced overexpression}

For overexpression of HSP-16.2, a transgenic line that drives GFP expression under the heat shock promoter hsp-16.2 was used. The microchamber $190 \mu \mathrm{m}$ x $190 \mu \mathrm{m}$ x $15 \mu \mathrm{m}$ 
with arrested L1 larvae were prepared as described above. In the first day of L1 arrest, worms in the S-basal chamber were transferred at $20^{\circ} \mathrm{C}$ to $37^{\circ} \mathrm{C}$ or $35^{\circ} \mathrm{C}$ for 1 hour or at $30^{\circ} \mathrm{C}$ for 3 hours. For the re-heat shock experiment, I heat shocked the 1 day arrested worm in chamber at $37^{\circ} \mathrm{C}$ for $1 \mathrm{~h}$ and keep the chamber at $20^{\circ} \mathrm{C}$ for 8 days. At the ninth day, the L1 larvae were re-heat shocked at $37^{\circ} \mathrm{C}$ for 1 hour and the GFP expression was re-measured. For measuring the GFP-expression of HSP-16.2, the worm was imaged directly in the chamber 15 min after completing the heat shock (preparation time for the correct microscope setting). I used Andor iXon (512x512 pixels) EMCCD camera combing with LED illumination (coolLed) and standard GFP filter sets. LED intensities were set to $20 \%$. The exposure time and the EM gain were set to $20 \mathrm{~ms}$ and 150 respectively. The $20 \mathrm{x}$ objective and a 0.7 lens were used in this experiment. Magnification was set to $1.5 \mathrm{x}$. The frame rate of the GFP-intensity changing was set to 4 frames/1h. Data were analyzed by Andor software. Threshold was set to 1000 and the integrated intensity per area unit $\left(\mu \mathrm{m}^{*} \mu \mathrm{m}\right)$ is represented in the figure. For calculating the difference of GFP-expression of HSP-16.2 between wild type and mutant, the mean expression of mutants at each time point was subtracted from the mean expression of wild type at the same time point. SE of the difference at each time point was computed using the method of error propagation [127]. Mann-Whitney U test was used for testing the significant difference of the mean expression.

\subsection{Quantification of bacteria consumption}

To measure the bacteria consumption of L1 worms for reaching the L2 stage, $10 \mathrm{ml}$ fresh OP50 culture was incubated at $37^{\circ} \mathrm{C}$ overnight. Afterward, the culture was centrifuged at 13,000×g for $60 \mathrm{~s}$. The pellet was washed with M9 buffer for 3 times and re-suspended in $1 \mathrm{ml} \mathrm{M} 9$ buffer. At OD 600 of 1.0, bacteria were calculated as $8 * 10^{8}$ cells $/ \mathrm{ml}$. Bacteria were further diluted to a concentration gradientand and pipetted on five $700 \mu \mathrm{m}$ x $700 \mu \mathrm{m}$ x $25 \mu \mathrm{m}$ (X length $\times$ Y length $\mathrm{x} Z$ depth) agarose microchambers respectively. The final bacteria cell number was calculated with the volume of each caption. I placed one synchronized L1 larva (1-day old) per caption and detected its developed stage by counting the molted skin in the closed caption of the chamber after 1 to 3 days. Dead worms were excluded from the final results. 


\subsection{ROS quantification}

To quantify the ROS level in the head region during starvation, arrested L1 were incubated in M9 buffer at $20^{\circ} \mathrm{C}$. Every 2 or 3 days, untreated worms were transferred to M9 containing $10 \mu \mathrm{M}$ DHE (Sigma, D7008) on a rotator for $30 \mathrm{~min}$ at $20^{\circ} \mathrm{C}$. and then immobilized with $25 \mathrm{mM}$ Levamisole on a $1 \mathrm{~mm}$ thick M9 4\% agarose pad [128]. Different $\mathrm{z}$ stacks of 10 to 15 worms were imaged respectively using a fluorescence microscope equipped with a spinning disc and an Andor iXon camera. The $488 \mathrm{~nm}$ laser (Andor Revolution on Nikon TiE) with 100\% intensity and 100x objective were used. EM gain of 100 and exposure time of $10 \mathrm{~ms}$ were set.

The obtained images were analyzed with Andor software. Therefore, we drew the head of the worm from pharynx to nose manually and used the maximum intensity Zprojection function to obtain the ROS intensity. The background was detected with a small square figure on the empty area of each image. The mean intensity of backgrounds was subtracted from the mean head intensity. To prevent the disturbing signal of autofluorescence and the mKate marker in RIS of the RIS ablated worms, we also imaged the untreated L1 larvae of each experiment, and used it as control. The mean intensity of the head area of the control was measured and subtracted from the mean head intensity of treated group.

\subsection{Fat content}

A fluorescent fatty acid analogue, C1-BODIPY 500/510 C12 (Invitrogen, D3823) was used to stain the intracellular fat bodies in L1 larvae [129]. Arrested L1 larvae were incubated in $49 \mathrm{nM}$ C1-BODIPY 500/510 C12 (1 mg/ml stock solution in DMSO, 1:50.000) in M9 buffer on the rotator for two days. Afterward, worms were immobilized with $25 \mathrm{mM}$ Levamisole on $4 \%$ agarose pads. A z-stack of $12 \mu \mathrm{m}$ in 41 planes was imaged with a spinning disc microcope (Andor Revolution on Nikon TiE) with a 488nm laser (with 25\% intensity and 60x oil objective. EM gain was set at 200 with the $100 \mathrm{~ms}$ exposure time. Untreated L1 larvae were imaged and served as control group.

The obtained images were processed and analyzed by the Andor iQ software. We drew the whole worm in the software and processed the images with an intensity Z-projection function to obtain the sum intensity of the BODIPY-fluorescence. The mean intensity of 
the background was determined with a small square figure on the empty area in each image and subtracted from the BODIPY-intensities. The auto-fluoresce intensity of the control worms was averaged and subtracted from the BODIPY-intensities.

\subsection{HSP-4 heat shock protein}

To quantify the expression of HSP-4 heat shock protein in starved L1 larvae, I used the strain SJ4005 phsp-4::GFP and crossed it with HBR227 aptf-1(gk794). L1 larvae were synchronized by bleaching and incubated in M9 at $20^{\circ} \mathrm{C}$. Arrested L1 larvae were dropped with M9 on the 4\% agarose pads and immobilized with $25 \mathrm{mM}$ Levamisol. The different $\mathrm{z}$ stacks $(27 \mu \mathrm{m}$ in 91 planes) of more than 10 worms were imaged using a fluorescence microscope equipped with a spinning disc and an Andor iXon camera. The $488 \mathrm{~nm}$ laser (Andor Revolution on Nikon TiE) was set to an intensity of $30 \%$ and the 40x objective was used. EM gain of 200 and exposure time of $30 \mathrm{~ms}$ were set. The obtained images were analyzed with Andor software. The whole worm was drawn manually in the software. I used the maximum intensity Z-projection function to obtain the whole worm phsp-4::GFP intensity. The background intensity was detected with a small square figure on the empty area of each image and subtracted in the software.

\subsection{Germ cells detection}

Signal of ppie-1::GFP was used to observe the division of germ cells (Z2 and Z3) in arrested worms [130, 131]. L1 larvae were synchronized by bleaching and incubated in M9 at $20^{\circ} \mathrm{C}$. Worms were dropped with M9 on the $4 \%$ agarose pads and immobilized with $25 \mathrm{mM}$ Levamisol. Z-stacks ( $15 \mu \mathrm{m}$ in 86 planes) of one worm were imaged using a fluorescence microscope equipped with a spinning disc and an Andor iXon camera. A $488 \mathrm{~nm}$ laser (Andor Revolution on Nikon TiE) with 100\% laser intensity and 100x objectives were used. EM gain and exposure time were set to 200 and $200 \mathrm{~ms}$ respectively. The number of germ cells at different times of the arrest was scored manually. To obtain the worm images after feeding, a Z-stack of $17 \mu \mathrm{m}$ in 86 planes was used. EM gain and exposure time were set to 200-300 and 200-250 ms respectively, depending on the GFP intensities. 


\subsection{Redox stage detection}

Two strains provided by Janine Kirstein [132] were used to test the redox levels of arrested L1: the F53B3.3roGFP is expressed in pan-neuronal cytosol and the unc54roGFP is expressed in muscular cytosol. L1 larvae were synchronized by bleaching and incubated in M9 at $20^{\circ} \mathrm{C}$. The L1 larvae were immobilized with $25 \mathrm{mM}$ Levamisol on $4 \%$ agarose pads. Worms were imaged with a multi-color-z-stack in 30 slices by using a fluorescence confocal microscope equipped with 40 x 0.95 objective (UPLSPO40x2). RoGFP levels in both neuronal and muscular cytosol were detected with two laser channels: $405 \mathrm{~nm}$ laser and $488 \mathrm{~nm}$ laser. Both channels were set to $50 \mathrm{~ms}$ exposure time. Excitation filter SEM-FF01-540/50, dichroic filter d1 quad 405/488/561/638 and emission filter FF01-534/43 A7 semrock were used in the microscope. For the image analysis we developed a new macro in Fiji to automated processing. The maximum intensity of the selected background area was subtracted individually from each slide in Fiji. Furthermore, a maximum intensity Z-projection function was used for the z-stack. The full body size of worm was drawn manually in the image and only the GFP intensity of the worm body was calculated to the mean intensity. The ratio of roGFP 405/488 emission was calculated by the division of the two mean intensities from each laser channel. More than 5 larvae were imaged at different arresting time points. Significance was tested using a two-sample t-test in OriginPro 2017.

\subsection{Statistics}

For the statistical tests in this thesis, we used Mann-Whitney U test, log-rank tests, Fisher's exact test and two-sample t-test using the softwares OriginPro 2017 (SR1 b9.4.1.354) or MATLAB R2017a (9.2.0.538062). We used the Benjamini-Hochberg Procedure test by $5 \%$ false discovery rate for multiple genotypes comparisons and multiple tissue-rescue comparison. 


\section{Results}

\subsection{Publication}

Wu Y*, Masurat F*, Preis J, Bringmann H. (2018) Sleep counteracts aging phenotypes to survive starvation-induced developmental arrest in C. elegans. Curr Biol. 28, 36103624. (* equal contribution). DOI: https://doi.org/10.1016/j.cub.2018.10.009

Together with Florentin Masurat, Jasmin Preis, and Henrik Bringmann, I published the paper in the journal Current Biology in 2018. I designed and performed the experiments of figure 4F, figure 5-6 and the supplemental figure S4-S6 as well as all the tables. I analyzed the data and created the figures for the experiments as mentioned above and further contributed to the manuscript writing the method part and the captions of figures. 


\section{Current Biology}

\section{Sleep Counteracts Aging Phenotypes to Survive Starvation-Induced Developmental Arrest in C. elegans}

Graphical Abstract

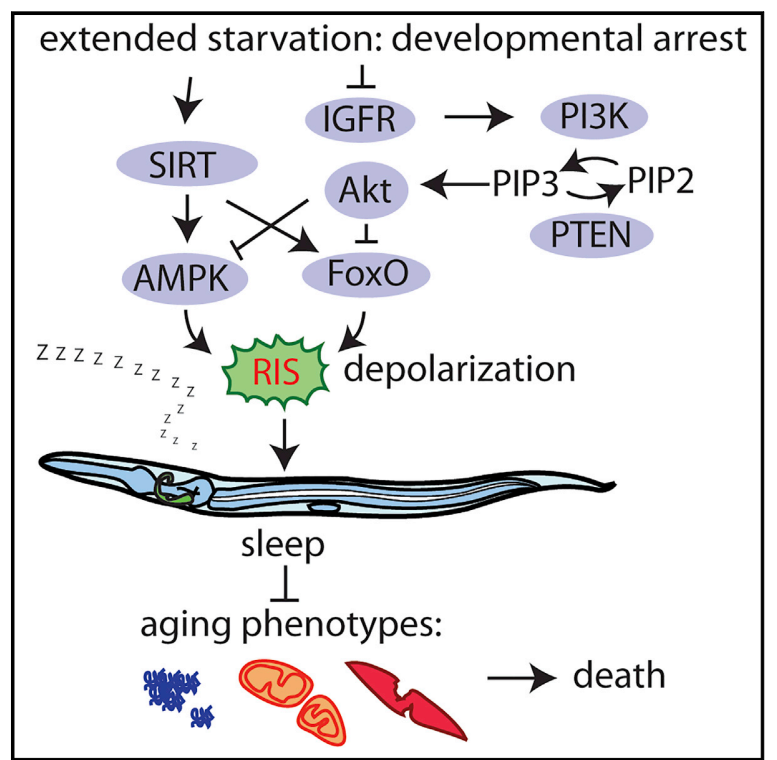

Highlights

- C. elegans sleep across most physiological conditions, including developmental arrest

- The sleep-active RIS neuron generally induces physiological sleep

- Insulin and sirtuin signaling control AMPK and FoxO to induce sleep during starvation

- Sleep is required to survive developmental arrest and counteracts aging phenotypes
Authors

Yin Wu, Florentin Masurat, Jasmin Preis, Henrik Bringmann

Correspondence

henrik.bringmann@mpibpc.mpg.de

In Brief

$\mathrm{Wu}$, Masurat, et al. show that C. elegans can sleep during most conditions and stages of their life. Worms sleep prominently during developmental arrest induced by starvation. A conserved aging gene network controls sleep through a sleep-active neuron, whose activity is required to slow the progression of aging phenotypes, thus allowing survival. 


\section{Sleep Counteracts Aging Phenotypes to Survive Starvation-Induced Developmental Arrest in C. elegans}

Yin Wu, ${ }^{1,2}$ Florentin Masurat, ${ }^{1,2}$ Jasmin Preis, ${ }^{1}$ and Henrik Bringmann ${ }^{1,3, *}$

${ }^{1}$ Max Planck Institute for Biophysical Chemistry, Am Fassberg 11, 37077 Göttingen, Germany

${ }^{2}$ These authors contributed equally

${ }^{3}$ Lead Contact

*Correspondence: henrik.bringmann@mpibpc.mpg.de

https://doi.org/10.1016/j.cub.2018.10.009

SUMMARY

Sleep is ancient and fulfills higher brain functions as well as basic vital processes. Little is known about how sleep emerged in evolution and what essential functions it was selected for. Here, we investigated sleep in Caenorhabditis elegans across developmental stages and physiological conditions to find out when and how sleep in a simple animal becomes essential for survival. We found that sleep in worms occurs during most stages and physiological conditions and is typically induced by the sleep-active RIS neuron. Food quality and availability determine sleep amount. Extended starvation, which induces developmental arrest in larvae, presents a major sleep trigger. Conserved nutrient-sensing regulators of longevity and developmental arrest, AMP-activated kinase and FoxO, act in parallel to induce sleep during extended food deprivation. These metabolic factors can act in multiple tissues to signal starvation to RIS. Although sleep does not appear to be essential for a normal adult lifespan, it is crucial for survival of starvation-induced developmental arrest in larvae. Rather than merely saving energy for later use, sleep counteracts the progression of aging phenotypes, perhaps by allocating resources. Thus, sleep presents a protective anti-aging program that is induced by nutrient-sensing longevity pathways to survive starvation-induced developmental arrest. All organisms are threatened with the possibility of experienced famine in their life, which suggests that the molecular coupling of starvation, development, aging, and sleep was selected for early in the evolution of nervous systems and may be conserved in other species, including humans.

INTRODUCTION

Sleep supports higher brain functions such as memory consolidation and synaptic plasticity [1]. Sleep disorders are linked to poor health, including the progression of neurodegenerative dis- eases and reduced lifespan in humans. Therefore, the wide prevalence of sleep disorders in modern societies poses a major health problem [2]. Sleep is ancient in origin and most likely evolved together with the emergence of a nervous system. However, little is known about the conditions that led to the evolution of sleep and about how sleep controls basic vital functions. Studying sleep in simple animals can shed light on the essential needs fulfilled by sleep [3].

Sleep is found in all organisms that have a nervous system, ranging from jellyfish to humans [4]. Its widespread occurrence implies that sleep is important, a view supported by the finding that sleep deprivation has detrimental effects [5]. Environmental conditions can impact sleep [6, 7]. Nutrient availability often fluctuates, and all organisms have thus established strategies to sense and respond to a lack of food. Across species, starvation triggers developmental arrest and a biphasic behavioral response consisting of a first phase of increased activity and suppressed sleep, followed by decreased physical activity [8-16]. Although increased physical activity is understood as a strategy to increase foraging, less is known about the regulation and function of decreased behavioral activity following long-term starvation [17]. Modest food deprivation can exert beneficial effects, suggesting that, in order to survive food deprivation, animals adapt their physiology and activate health- and longevity-promoting pathways $[16,18]$

Caenorhabditis elegans is a model animal with low ethical hurdles for harsh survival assays. It lives a boom-and-bust lifestyle with periods of rapid proliferation when food is present alternating with long periods of starvation. As an adaptation to food scarcity, C. elegans has evolved survival strategies, including larval developmental arrest, an alternative larval life stage called "dauer," and an increased lifespan in adults [19-21]. The study of dietary restriction and starvation and their role on lifespan in $C$. elegans has led to the identification of major conserved signaling pathways controlling development and aging. Important nutrient-sensing and lifespan-promoting systems act through AMP-activated kinase and the FoxO transcription factor [21].

Like most animals, C. elegans sleeps, a phenomenon that has been studied most in the developing larva and after cellular stress in the adult $[22,23]$. From worms to humans, sleep is induced by conserved sleep-active neurons that depolarize at the onset of sleep. They actively induce this behavior by directly inhibiting arousal circuits through GABA and neuropeptides. 
A
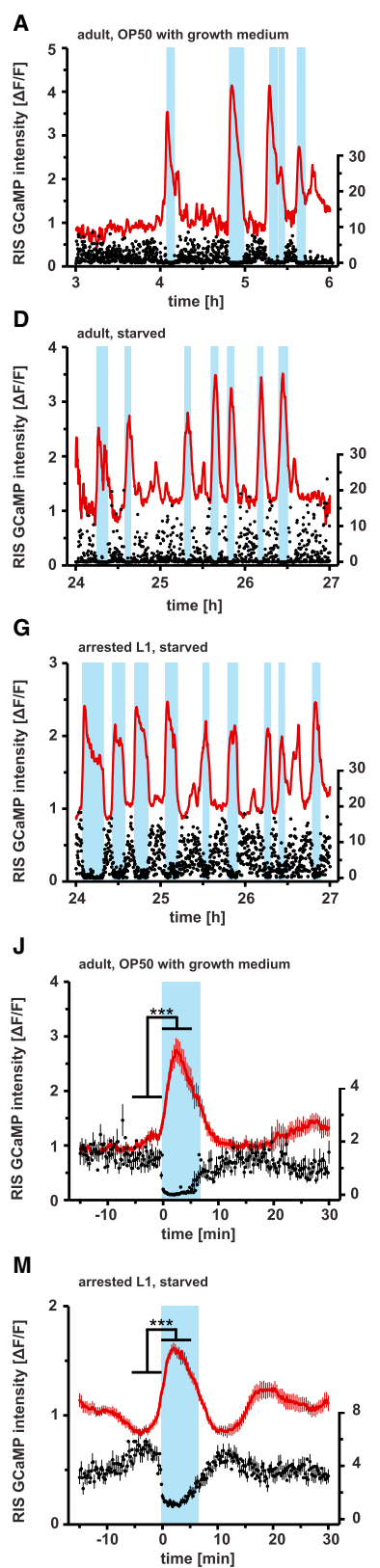
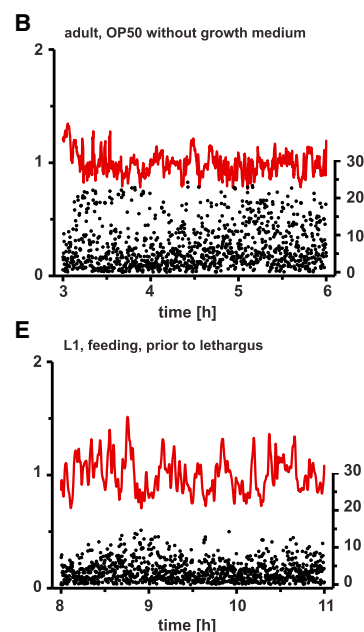

H

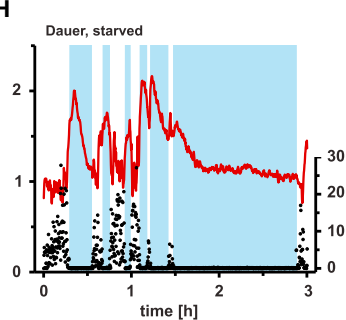

k

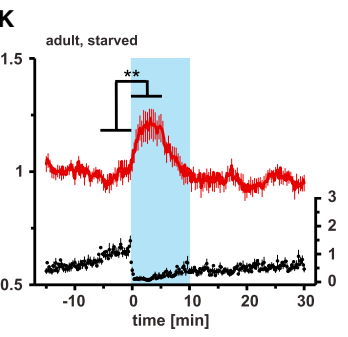

N

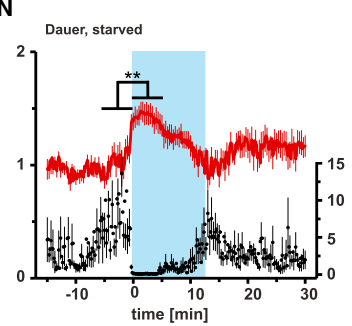

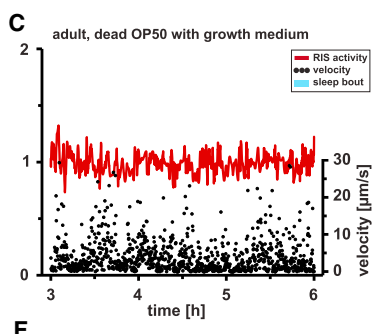
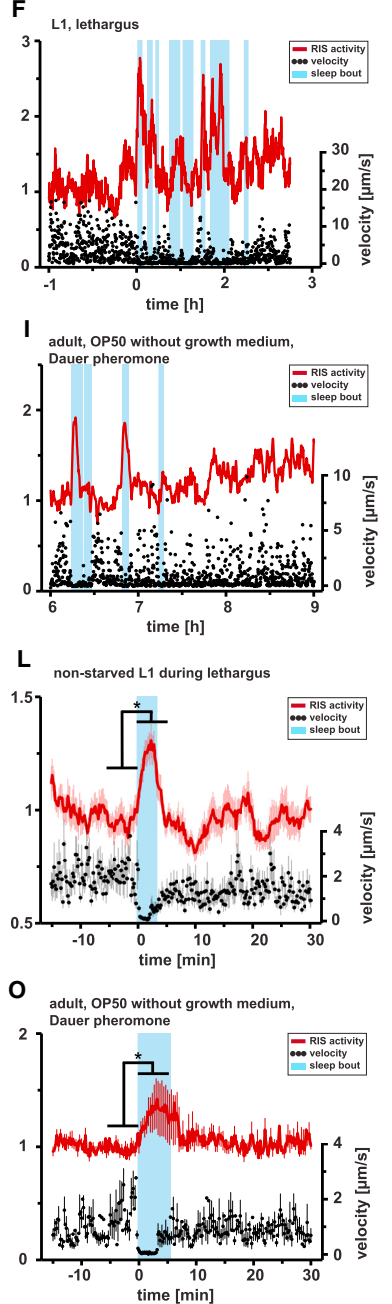

(legend on next page)

Current Biology 28, 3610-3624, November 19, 20183611 
Upstream pathways control the depolarization of sleep-active neurons and thereby control the timing and amount of sleep $[24,25]$. In mammals, several populations of sleep-active neurons exist, which are thought to induce sleep in a concerted action. The best-studied population of sleep-active neurons is found in the preoptic area (POA). These neurons form part of the so-called descending system that inhibits arousal during sleep. Sleep-active neurons also confer an increased sleep pressure after sleep deprivation, as their depolarization is increased at the onset of rebound sleep [26, 27]. Similarly, several populations of sleep-promoting neurons exist in Drosophila. A cluster of nerve cells innervating the dorsal-fanshaped body (dFB) of the central complex presents a well-studied population of sleep-promoting neurons, whose excitability can be switched dependent on sleep need [28]. In C. elegans, sleep during lethargus, a developmentally controlled phase of molting during which worms synthesize a new cuticle, requires a single sleep-active sleep-inducing neuron called RIS, which expresses GABA and inhibitory RFamide peptides. Like its mammalian counterparts, this neuron induces sleep when depolarized optogenetically, shows calcium transients at sleep onset, most likely indicating depolarization, shows over-activation after sleep deprivation, and is inhibited by waking stimul [29-31].

To find out why a simple animal sleeps, we searched for conditions in which RIS-induced sleep is essential for survival in C. elegans. Although worms show sleep during most stages and conditions, this behavior is most prominent during various forms of starvation and developmental arrest, where conserved nutrient-sensing lifespan regulators control it. Although sleepless adult worms have a normal lifespan under both ample food condition as well as starvation, sleeplessness impairs survival of larvae during developmental arrest. Interestingly, the role of sleep in starvation-induced arrest survival appears not to only be conservation of energy but to prevent the progression of aging phenotypes. Thus, sleep presents an adaptive anti-aging strategy to survive starvation-induced arrest. Ou work provides a molecular link between sleep, longevity, starvation, and developmental arrest with high potential implications for the evolutionary origin of sleep as well as for human health.

\section{RESULTS}

Sleep Is Widespread across Stages and Conditions, with Extended Starvation Presenting a Major Sleep Trigger To find out how sleep becomes vital in a simple animal, we quantified sleep during several life stages and conditions to find out what is the strongest trigger for this state. We focused on food availability and quality, which affects behavioral activity and quiescence [10, 11, 19, 32, 33]. We used RIS calcium imaging as a proxy for depolarization of this neuron and locomotion quiescence as an assay to identify and quantify sleep. Worms were cultured in microfluidic devices made from hydrogel $[34,35]$ and behavior, and calcium transients in RIS were imaged and quantified during different life stages and food conditions (Figures 1 and S1). We first looked for sleep in adult worms and tested the effects of three types of bacterial food: first, to mimic standard worm culture conditions, we fed worms with bacteria in the presence of bacterial growth medium [32] (Figure $1 \mathrm{~A}$ ). Second, we tested bacteria that were depleted of growth medium (Figure 1B). And third, we tested dead bacteria in the presence of growth medium (Figure 1C). Worms living on fed bacteria showed extended sleep bouts (Figures 2A, S2A, and S2B). A reduction of locomotion always coincided with increased RIS depolarization (Figure 1J). Worms feeding on dead or starved bacteria, however, showed virtually no sleep behavior (Figures 1B, 1C, and 2A).

Short-term (few hours) fasting results in increased arousal and foraging in C. elegans [15], whereas prolonged (more than $12 \mathrm{hr}$ ) starvation results in behavioral quiescence and developmental arrest $[10,11,19,20,33,36]$. To analyze sleep during starvation and arrest, we looked at one day starved adults, L1 larvae during lethargus (when worms do not feed during cuticle remodeling), one day starved developmentally arrested L1 larvae, and three days starved dauer larvae. We observed prominent sleep bouts during starvation and arrest in all stages that were tested (Figures 1D-1H, 2B-2E, and S2C-S2J). RIS again depolarized strongly at the onset of all sleep bouts (Figures $1 \mathrm{~K}-1 \mathrm{~N}$ ).

As pheromones play a strong role in anticipation of adverse life conditions and the development of the developmentally arrested dauer larva [37], we also tested the effect of pheromones on sleep in adults feeding on starved bacteria. Dauer pheromone

Figure 1. Food Conditions Control Sleep Amount in C. elegans across Stages

(A-l) Individual RIS calcium imaging and sleep measurements. RIS activity is shown in red and locomotion speed in black; blue shading shows sleep bouts as defined by a locomotion cessation threshold. At the onset of a sleep bout, RIS activated and locomotion ceased.

(A) An adult worm feeding on OP50 bacteria with bacterial growth medium.

(B) An adult worm feeding on OP50 without bacterial growth medium.

(C) An adult worm feeding on dead OP50 with growth medium.

(D) An adult that was starved for $24 \mathrm{hr}$

(E) An L1 larva in the presence of food, $8 \mathrm{hr}$ after hatching and prior to lethargus.

(F) An L1 larva before and during lethargus as defined as the non-feeding period (starting at $0 \mathrm{hr}$ ) prior to the molt in the presence of food.

(F) An L1 larva before and during lethargus as defined

(G) An arrested L1 larva that was starved for $24 \mathrm{hr}$.

(H) A 3-day-old dauer larva in the absence of food.
(I) An adult feeding on OP50 without growth medium in the presence of dauer pheromone.

$(\mathrm{J}-\mathrm{O}) \mathrm{RIS}$ activity increased significantly with locomotion cessation at the onset of sleep bouts in all conditions. The increase of $\Delta \mathrm{F} / \mathrm{F}$ of the calcium sensor signal was $(J) 126.1 \% \pm 20.2 \%, n=24$ worms, ${ }^{* \star} p<0.001$ for adults on growing OP50; (K) $32.5 \% \pm 5.4 \%, n=20$ worms, ${ }^{\star \star} p<0.01$ for starving adults; and (L) $22.0 \% \pm$ $0.9 \%, n=14$ worms, ${ }^{*} \mathrm{p}=0.014$ for developing $\mathrm{L} 1$ worms during lethargus.

(M-O) $58.7 \% \pm 4.9 \%(M), n=33$ worms, ${ }^{* * *} p<0.001$ for arrested L1 larvae; $36.8 \% \pm 7.9 \%(N), n=13$ worms, ${ }^{* *} p=0.002$ for dauer larvae; $45.7 \% \pm 19.8 \%(O)$, $\mathrm{n}=12$ worms, ${ }^{*} \mathrm{p}=0.02$ for adults on stationary OP50 with dauer pheromone.

For all statistical comparisons, the paired Wilcoxon rank test was used. See also Figures S1 and S2.

3612 Current Biology 28, 3610-3624, November 19, 2018 
A

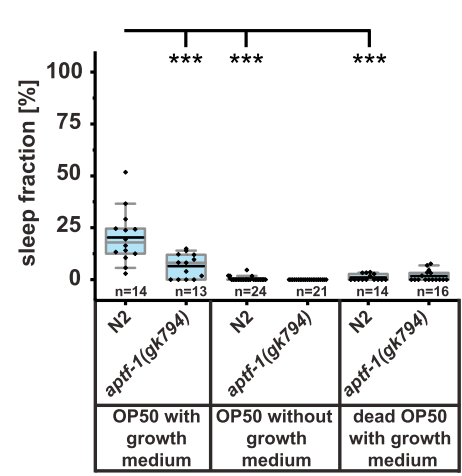

B

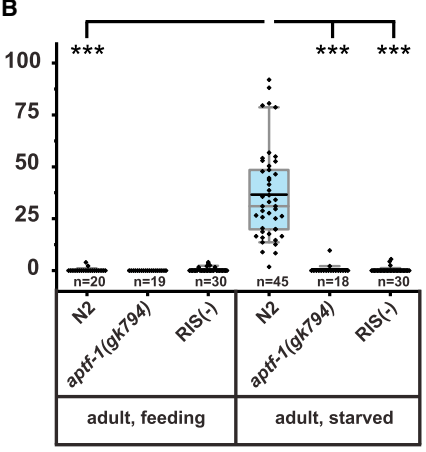

C

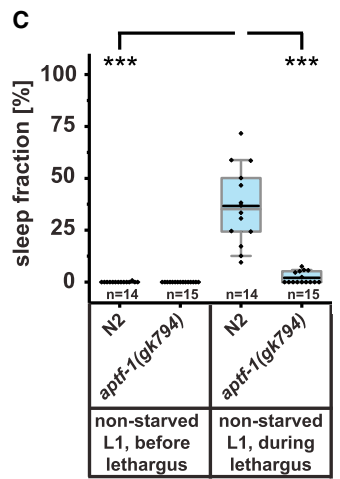

D

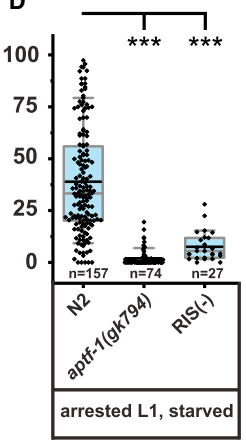

E

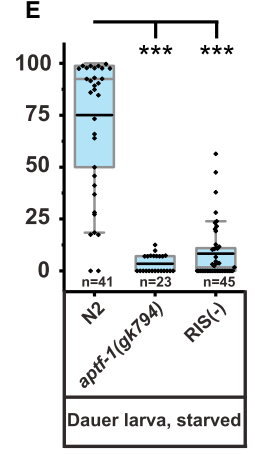

F

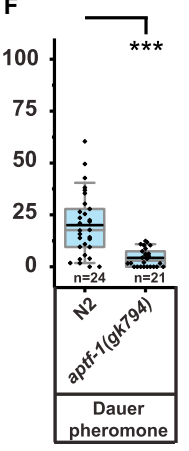

Figure 2. The Sleep-Active RIS Neuron Is Required for All Types of Physiological Sleep Fraction of time spent asleep in wild-type, aptf-1 (gk794), and RIS-ablation (RIS(-)).

(A) Median sleep duration in adult worms in the presence of growing bacteria was $18 \%$ in wild-type and $8 \%$ in aptf-1(gk 794$) ; * \star *<<0.001$. In the absence of growth medium and in the presence of dead bacteria, median sleep duration was $0 \%$ for both wild-type and aptf-1(gk794).

(B) In adult worms, starvation increased sleep compared with worms fed on bacteria in the absence of growth medium. Median sleep duration in starved adults was $31 \%$ in wild-type and $0 \%$ in aptf- 1 (gk794) and RIS(-); *** $\mathrm{p}<0.001$.

adults was $31 \%$, wild-type and $0 \%$ in apt-1(gk79) and both wild-type and aptf-1(-). During lethargus, the median sleep fraction was $35 \%$ for wild-type and $0 \%$ for aptf-1(gk794); ${ }^{* \star} p<0.001$.

(D) Sleep fraction in starved L1 larvae. Wild-type larvae had a median sleep fraction of 33\%, aptf-1(gk794) $0 \%$, and RIS(-) $5 \%$; ***p $<0.001$.

(E) Sleep fraction in dauer larvae. Median sleep fraction in dauer larvae was $92 \%$ wild-type, $0 \%$ in aptf-1(gk794), and $2 \%$ in RIS $(-) ;$; $* * 0<<0.001$.

(F) Sleep fraction in adult worms in the presence of dauer pheromone. Wild-type worms showed a median sleep fraction of $18 \%$ and aptf-1(gk794) of $3 \%$; ${ }_{* \star *} \mathrm{p}<0.001$.

The numbers of assayed worms $(n)$ are displayed below the boxplots. Statistical comparisons were made using the Mann-Whitney $\mathrm{U}$ test.

extract also induced sleep bouts and RIS depolarization, which is consistent with the observation that population density affects sleep (Figures 1I, 10, 2F, S2K, and S2L) [16].

In summary, behavioral quiescence was typically accompanied by RIS activation at the onset of virtually all sleep bouts and across all stages and conditions that we tested. An increase in RIS calcium precisely correlates with reduction of behavioral activity. Thus, rather than reflecting sleep pressure building up during wakefulness prior to sleep onset [11, 38], RIS activity indicates the active induction of sleep. Food conditions appear to be a major determinant for RIS activation and sleep amounts in both larvae and adults. Not only the presence or absence of food determines sleep quantity but also food quality, with metabolizing bacteria appearing as a strong sleep trigger. Extended starvation and arrested development triggered the strongest sleeping behavior. As pheromones signal population density, sleep may already be modulated in anticipation of starvation. RIS calcium imaging combined with locomotion quantification thus presents a straightforward assay to identify sleep, which occurred during all stages and most conditions and thus is much wider spread than previously thought $[22,39]$.

Current Biology 28, 3610-3624, November 19, 20183613 
A

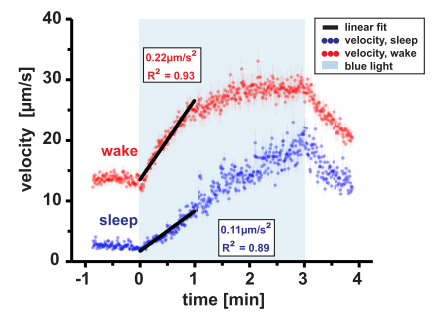

C
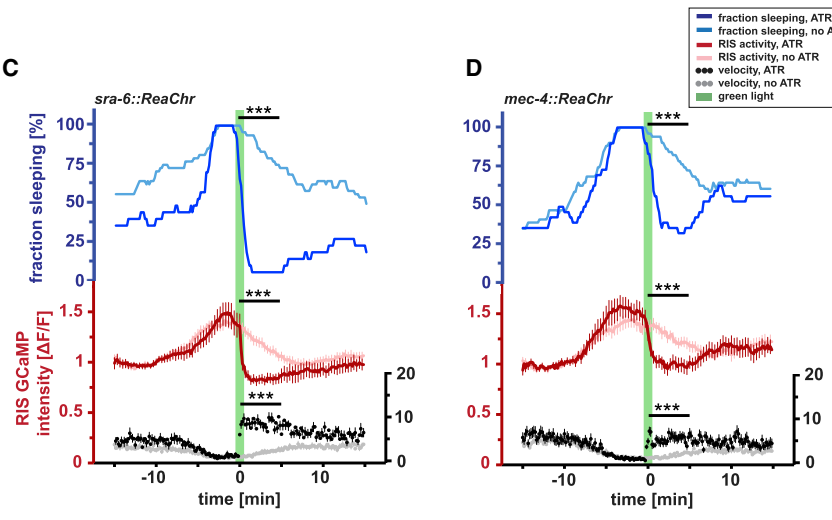

B

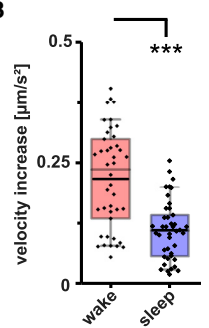

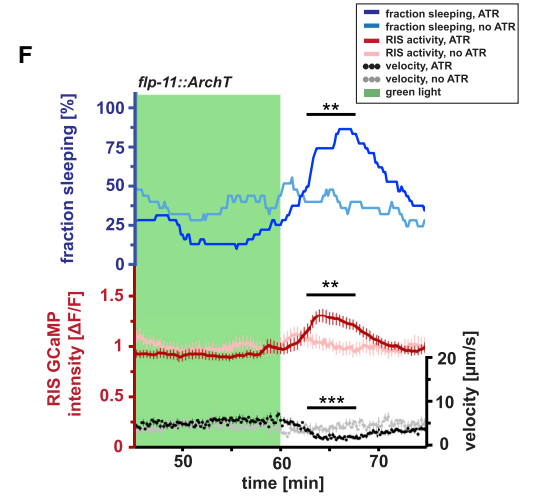

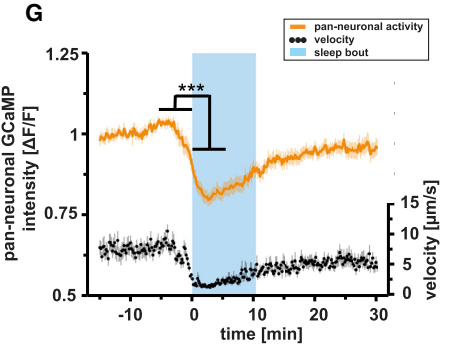

Figure 3. Behavioral Quiescence during L1 Arrest Presents a Sleep State

(A and B) To test for responsiveness to stimulation during quiescence in arrested L1 larvae (A), a blue light stimulus was given and locomotion velocity was measured. (B) Linear regression of locomotion speeds during the first minute of blue light irradiation was used to measure locomotion acceleration. Waking worms (red dotted line, red box) accelerated with $0.22 \pm 0.02 \mu \mathrm{m} / \mathrm{s}^{2}$, whereas sleeping worms (blue dotted line, blue box) accelerated only with $0.11 \pm 0.01 \mu \mathrm{m} / \mathrm{s}^{2}$ $\left(\mathrm{n}=42\right.$ worms; ${ }^{* * *} \mathrm{p}<0.001$; paired Wilcoxon rank test).

(C-E) To test for sleep reversibility, nociceptive ASH neurons and mec-4 expressing mechano-sensory neurons were stimulated optogenetically with ReaChr and green light in sleeping arrested L1 larvae. In addition, arrested L1 larvae were stimulated with noxious blue light. RIS activity is shown in red (control without all trans-Retinal [ATR] in light red), speed in black (control without ATR in gray), and the fraction of sleeping animals in blue (control without ATR in light blue). 
Across Life Stages and Physiological Conditions, Sleep Is Induced by the Sleep-Active RIS Neuron

RIS is crucially required for sleep during lethargus [29, 30]. During un-physiological conditions, such as after severe cellular stress, a second neuron, called ALA, has been suggested to induce sleep independently of RIS [39-41]. Our imaging results suggest that sleep, at least under more physiological conditions, generally involves the RIS neuron. We hence tested whether the types of sleep that we observed depend on RIS. To ablate RIS function, we used aptf-1 mutant worms that lack neuropeptide expression in RIS, which is required for lethargus sleep induction. In addition, we genetically ablated RIS by expression of the apoptosis inducer EGL-1 from a RIS-specific promoter [30]. In RIS-deficient worms, sleep was strongly reduced or virtually abolished (median sleep fraction in fed adult worms with growth medium was reduced by $56 \%$ in aptf-1(gk794); in starved adults by $100 \%$ in aptf-1(gk794) and RIS(-); during lethargus by $100 \%$ in aptf-1 (gk794); in arrested L1 larvae by $100 \%$ and $84 \%$ in aptf-1(gk794) and RIS(-), respectively; in dauer larvae by $100 \%$ and $98 \%$ in aptf-1 (gk794) and RIS(-), respectively; and in adults in the presence of dauer pheromone by $83 \%$ in aptf-1(gk794); Figure 2). Thus, consistent with our imaging data, RIS is required for sleep under all studied conditions. This suggests a pivotal role of RIS in sleep under physiological conditions.

Starvation-Induced Quiescence during L1 Arrest Is a Sleep State

The dependency of quiescence on RIS indicates that most types of behavioral quiescence in the worm constitute sleep rather than other types of quiescence, such as quiet wakefulness or paralysis. However, quiescence during L1 arrest has not yet been characterized as sleep using behavioral criteria $[10,11,19,32$ $33,41-43]$. Sleep is defined and can be distinguished from other types of quiescence by a reduced response threshold, rapid reversibility, and homeostatic regulation [44]. We hence assayed whether quiescence during L1 arrest fulfills the behavioral definition of sleep.

To test for responsiveness, we used blue light to trigger an escape response [45] in arrested L1 larvae inside micro- fluidic compartments and measured the resulting increase in locomotion. Although waking worms responded with a strong locomotion increase, during sleep, the acceleration was reduced (Figures $3 \mathrm{~A}$ and $3 \mathrm{~B}$ ). We next tested for reversibility of sleep by providing a strong optogenetic sensory stimulus. For this, we expressed the channelrhodopsin variant ReaChR in nociceptive ASH neurons or mechano-sensory neurons, depolarized them with green light [46], and followed locomotion and RIS polarization. Optogenetic stimulation of $\mathrm{ASH}$ neurons or $\mathrm{mec}-4$-expressing neurons quickly reversed quiescence (Figures 3C, 3D, S3A and S3B). The waking stimulus also acutely inhibited RIS activity, leading to a premature termination of the RIS depolarization transient (Figures 3C, 3D, S3A, and S3B). To provide a non-optogenetic sensory stimulus, we applied noxious blue light, which triggers an avoidance response [45, 47]. Similar to the optogenetic stimulus, blue light reversed quiescence and inhibited RIS (Figures 3E and S3C).

To test for homeostatic regulation, we deprived sleep through temporary optogenetic inhibition of RIS using the light-driven proton pump ArchT [48]. We inhibited RIS for $1 \mathrm{hr}$ using green light and measured the behavioral response and the depolarization of the RIS neuron before, during, and after the inhibition. Optogenetic silencing efficiently prevented RIS depolarization and sleep induction during illumination (Figures S3D and S3E) Following the end of RIS inhibition, sleeping behavior and RIS depolarization increased compared to control levels, suggesting that quiescence is under homeostatic control (Figures 3F, S3D and S3E).

During sleep, including $C$. elegans lethargus, the majority of neurons show dampened activity [38, 49]. To test whether global neural activity is reduced during sleep, we imaged pan-neurona calcium activity. Global brain activity was indeed dampened during quiescence bouts (Figure $3 G$ ).

Thus, starvation-induced quiescence during larval arrest presents a sleep state, with its key behavioral and neurophysiological hallmarks. Regulatory principles typical for mammalian sleep are present also in starvation-induced sleep: the fast inhibition of RIS through a waking stimulus is similar to the flip flop switch that ensures discrete behavioral states and the increased RIS

(C) Sleeping worms responded immediately to ASH activation and the sleep bout ceased. The fraction of sleeping animals decreased by $91.6 \% \pm 1.7 \%$ (contro worms without ATR by $12.3 \% \pm 2.9 \%$; ${ }^{* \star *} \mathrm{p}<0.001$; two-sample $\mathrm{t}$ test). RIS activity $(\Delta \mathrm{F} / \mathrm{F}$ ) dropped by $35.5 \% \pm 4.2 \%$ (control worms without ATR showed only a drop of $\Delta F / F$ of $11.9 \% \pm 0.3 \% ; * * x<0.001$ two-sample $t$ test). Locomotion velocity increased by $313.7 \% \pm 26.0 \%$ (control worms without ATR by $65.7 \%=$ $14.0 \% ;{ }^{* * *} p<0.001 ;$ two-sample $t$ test). $n_{(\text {ATR })}=23$ worms; $n_{\text {(without ATR) }}=47$ worms.

(D) Sleeping worms immediately responded to mec-4-expressing mechano-sensory neuron activation, and the sleep bout ceased. The fraction of sleeping animals decreased by $52.45 \% \pm 6.0 \%$ (control worms without ATR by $10.0 \% \pm 2.6 \%$; *** $\mathrm{p}<0.001$; two-sample t test). RIS activity ( $\Delta \mathrm{F} / \mathrm{F}$ ) dropped by $32.8 \% \pm$ $3.2 \%$ (control worms without ATR showed only a drop of $\Delta \mathrm{F} / \mathrm{F}$ of $7.0 \% \pm 2.1 \%$; ${ }^{* * *} \mathrm{p}<0.001$; two-sample $\mathrm{t}$ test). Locomotion velocity increased by $351.7 \% \pm$ $44.0 \%$ (control worms without ATR by $14.6 \% \pm 4.0 \%$; ${ }^{* *} \mathrm{p}<0.001$; two-sample $t$ test). $n_{(\text {ATR })}=29$ worms; $n_{\text {(without ATR) }}=50$ worms.

(E) Sleeping worms immediately responded to noxious blue light illumination, leading to sleep bout termination. The fraction of sleeping animals decreased by $75.5 \%+3.5 \%$ (control worms without blue light stimulation by $4.1 \%+0.6 \%$; *** $<0.001$; two-sample $t$ test). RIS activity ( $\Delta F / F$ ) dropped by $43.2 \% \pm 7.9 \%$ $7.5 \% \pm 3.5 \%$ (cth (control worms without blue light stimulation showed only a drop of $\Delta F / F$ of $4.3 \% \pm 7.7 \%$; " $p<0.001$; two-sample $t$ test). Locomotion velocity increased by $367.9 \% \pm 5.8 \%$ (control worms without ATR by $80.3 \% \pm 28.2 \%$; ${ }^{* *} \mathrm{p}<0.001$; two-sample $\mathrm{t}$ test). $\mathrm{n}_{\text {(stimulation) }}=35$ worms; $\mathrm{n}_{\text {(without stimulation) }}=37$ worms. (F) To test for homeostasis, RIS was inhibited for $1 \mathrm{hr}$ with ArchT and green light in the arrested L1 larvae. RIS activity is shown in red (control without ATR in light red), speed in black (control without ATR in gray), and the fraction of sleeping animals in blue (control without ATR in light blue). After the end of green ligh exposure, the fraction of sleeping worms increased by $265.6 \% \pm 13.1 \%$ (control worms without ATR decreased by $19.3 \% \pm 6.7 \%$; ** $\mathrm{p}=0.007$; two-sample $t$ test). RIS activity ( $(\mathrm{F} / \mathrm{F}$ ) increased by $24.3 \% \pm 2.2 \%$ (in control worms without ATR, it increased by $5.6 \% \pm 1.0 \%$; ** $\mathrm{p}=0.005$; two-sample $t$ test). Locomotion velocity decreased by $63.3 \% \pm 16.0 \%$ (control worms without ATR increased by $5.5 \% \pm 4.0 \% ; * * * 0<0.001$; two-sample t test). $n_{(\text {ATR })}=32$ worms; $n_{(\text {(without ATR) }}=25$ worms. (G) To test for overall neuronal activity during a sleep bout, calcium activity in the head neurons was measured using pan-neuronal GCaMP6s. Head neuron activity is shown in orange and locomotion speed in black; blue shading shows sleep bouts as defined by a locomotion cessation threshold. At the onset of a sleep bout, head neuron activity decreased and locomotion ceased. $\Delta F / F$ was decreased to $82.3 \% \pm 0.2 \%$ ( $n=21$ worms; ${ }^{* * *} p<0.001$; paired Wilcoxon rank test). Error bars in (C)-(G) indicate the SEM. See also Figure S3. 
A

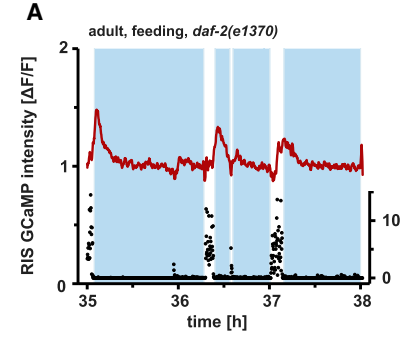

D

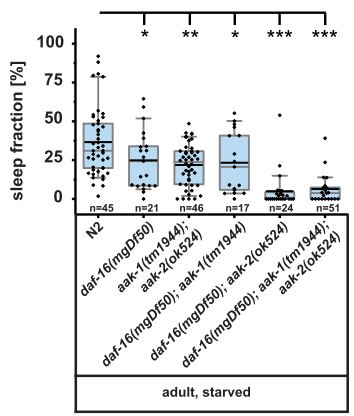

B

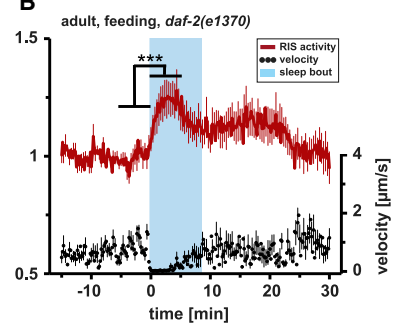

E

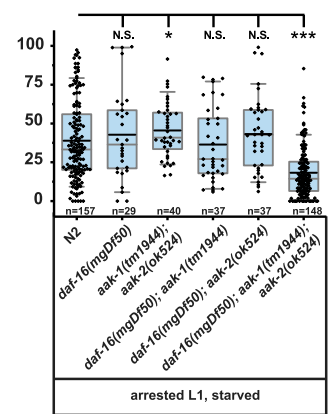

c

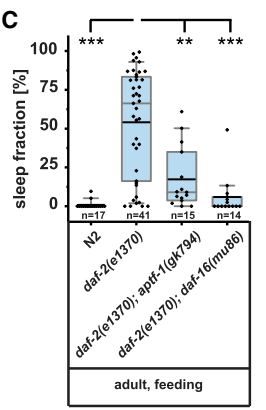

F

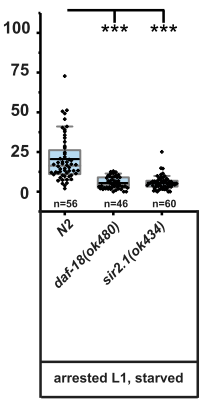

G

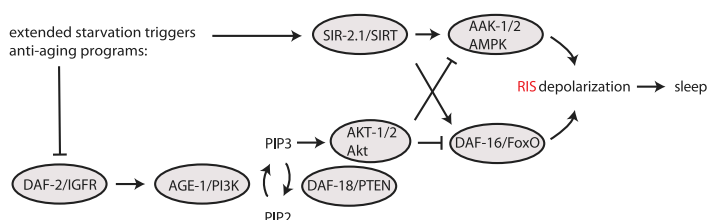

Figure 4. The Nutrient-Sensing Longevity Regulators IIS and AMP Kinase Regulate Starvation Sleep through RIS Activation

(A) RIS GCaMP3 signal intensities and corresponding velocity traces of a representative daf-2(e1370) mutant worm. RIS activity is shown in red and locomotion speed in black; blue shading shows sleep bouts as defined by a locomotion cessation threshold. At the onset of a sleep bout, RIS activated and locomotion ceased. Note that RIS depolarizes at sleep onset and is not depolarized during the entire quiescence bout, which is similarly observed in the dauer arva.

(B) Averaged RIS activity and velocity aligned to sleep bout onset. RIS activity ( $\Delta F / F)$ increased by $17.9 \% \pm 4.7 \%$ during sleep $\left(n=15\right.$ worms; ${ }^{* * *} p<0.001$; paired Wilcoxon rank test).

(C) DAF-2 inhibition induces sleep through RIS and DAF-16. Median sleep time was 66\% in daf-2(e1370); $9 \%$ in daf-2(e1370); aptf-1(gk794), ${ }^{* *} p<0.01$; and $0 \%$ in daf-2(e1370); daf-16(mu86), ${ }^{* * *} \mathrm{p}<0.001$.

(D) Longevity genes control starvation-induced sleep in the adult: median time spent in sleep was $31 \%$ in wild-type; $24 \%$ in daf-16(mgDf50) mutation, ${ }^{*} p<0.05$; 24\% in aak-1(tm1944);aak-2(ok524) double mutants, " ${ }^{*} \mathrm{p}<0.01 ; 21 \%$ in daf-16(mgDf50)/aak-1(tm1944) double mutants, " $\mathrm{p}<0.05 ; 0 \%$ in daf-16(mgDf50)/ aak-2(ok524) double mutants, ${ }^{* \star *} \mathrm{p}<0.001$; and $4 \%$ in daf-16(mgDf50)/aak-1(tm 1944)/aak-2(ok524) triple mutants, ${ }^{* \star *} \mathrm{p}<0.001$.

(E) Longevity genes control starvation-induced larval sleep. Single or double mutation combinations did not reduce sleep significantly, but, compared with wild-type (median sleep fraction 33\%), the daf-16(mgDf50)/aak-1(tm1944)/aak-2(ok524) triple mutant reduced sleep (median sleep fraction $14 \%$ ); ${ }^{*} p<0.05$; wild-type (me

(F) Insulin signaling and sirtuin, which control the activity of FoxO and AMPK, control sleep. Median sleep fraction was $16 \%$ in wild-type; $4 \%$ in daf-18(ok480), ${ }^{* \star *} \mathrm{p}<0.001$; and $5 \%$ in sir-2.1(ok434), ${ }^{* * *} \mathrm{p}<0.001$. 
depolarization during rebound sleep also occurs similarly in sleep-active neurons of the vertebrate POA [26].

AMP-Activated Kinase and FoxO Act in Parallel to Induce Sleep during Starvation

Starvation is sensed by conserved pathways that trigger protective strategies. AMP-activated kinase (AAK-1/2) is an energy sensor that is activated by a high AMP/ATP ratio and that triggers the switch from anabolic to catabolic processes. Reduced activity of the insulin/insulin-like signaling (IIS) receptor (DAF-2) leads to the activation of FoxO (DAF-16), a transcription factor that expresses genes that promote stress resistance and longevity [21]. DAF-16 also plays a role in coping with the consequences of stressful sleep deprivation and modulates quiescence [11, 50-52]. Both FoxO and AMPK are required for starvationinduced developmental arrest [20,53]. To test whether longevity pathways are responsible for the majority of starvation sleep, we measured RIS activation and sleep in mutants that are defective in AMP-activated kinase and IIS.

We first tested a loss-of-function mutation in daf-2 in the presence of food conditions that normally do not lead to sleep. daf-2(-) caused sleep and RIS depolarization in the presence of starved bacteria as a food source (Figures 4A-4C, S2M, and $\mathrm{S} 2 \mathrm{~N}$ ), and this effect was dependent on daf-16 (Figure 4C). We next tested sleep in daf-16(-) during larval and adult starvation. daf-16(-) showed only a moderate reduction of sleep in adults, consistent with previous results (Figure 4D) [11]. Similarly, partially reduced sleeping behavior was found in aak-1/aak-2 double-mutant animals during starvation (Figure 4D), prompting us to knock out both pathways simultaneously. The aak-2/daf-16 double mutant showed a near-complete absence of sleep in the adult (Figure 4D), but not in the larva (Figure 4E). aak-1/aak-2/daf16 triple mutant worms showed a strong loss of sleep in the starved adult (Figure 4D) and also a strong sleep reduction in the arrested L1 larva (Figure 4E). Thus, AAK-1/2 and DAF-16 appear to act in parallel to induce sleep during extended starvation.

We next wished to link starvation-induced sleep to the genes that are upstream of AMPK and FoxO. We hence quantified sleep in mutants of genes that are known to control AMPK and FoxO. In the presence of food, IIS activates the phosphatidylinositol 3-kinase (PI3K) AGE-1 to produce PIP3, which activates Akt kinase (AKT-1/2) [21]. A major role of Akt in C. elegans is to inhibit FoxO [54]. In other systems, Akt also inhibits AMPK by adding an inhibitory phosphorylation on Ser485, which then inhibits the activation of AMPK by LKB1 [55], and there is evidence that Akt also inhibits AMPK in C. elegans [56]. Thus, both FoxO and AMPK have in common that they can be suppressed through Akt [55-57]. The tumor suppressor DAF-18/PTEN inhibits IIS signaling by hydrolyzing PIP3 produced by AGE-1/PI3K. daf-18 deletion thus leads to constitutive activation of Akt [21]. We tested whether daf-18 deletion leads to a suppression of starvation-induced sleep and found that sleep was strongly reduced (Figure 4F). Starvation also leads to the activation of SIRT/
SIR-2.1, which can act through activation of both FoxO [57] and AMPK [58]. We thus tested sleep after deletion of sir-2.1. We observed again a reduction of sleep in sir-2.1(-) mutants (Figure 4F). These results are consistent with the known network of longevity genes and a model in which, in the presence of food, canonical IIS is wake promoting through the inhibition of AMPK and FOXO and in which, during starvation, sirtuin signaling is sleep-promoting by activating AMPK and FOXO (Figure 4G). Such dual control mechanism of two parallel pathways could account for the robustness of sleep induction during starvation. Our data thus suggest that the known network of longevity genes controls sleep induction during starvation.

To find out in which tissues AMPK and FoxO act during starvation to induce sleep, we performed rescue experiments. We used daf-2(-)/daf-16(-) worms and expressed daf-16 specifically in either neurons, muscle, or intestine and quantified sleep in adults in the presence of food $[59,60]$. Sleep was reinstated partially but significantly by expression in muscle and inconsistently by expression in intestine, but not by expression in neurons (Figure S4A). The role of daf-16 in muscle during starvation is consistent with previous data suggesting a role of daf- 16 in muscle to control increased sleep after forced locomotion during lethargus and supports a conserved role of muscle in signaling sleep need $[51,61]$. During physical exercise, musculature is the major consumer of energy, and this tissue may thus be well suited to signal energy shortage during both forced movement and starvation [62]. The weak rescue from non-neuronal tissues together with the lack of neuronal rescue suggests that daf-16 acts in signaling centers outside of RIS.

To identify the tissues in which AMPK acts, we expressed aak-2 in an aak-2(-)/daf-16(-) background. We quantified sleep in adult worms in the absence of food and looked for rescue in neurons, muscle, intestine, hypodermis, and the excretory cell $[15,56,63]$. AMPK rescue effects were partial but significant in all tissues that were tested. Because AMPK could be rescued from all tissues, including neurons, we specifically tested whether AMPK also can act in RIS and found that it partially could (Figures S4B and S4C). Thus, AMPK appears to be able to induce sleep by acting in many tissues, including in RIS. Consistent with previous reports, which found that AMPK can act across various tissues, this may indicate that many or perhaps even all tissues that experience energy stress can signal to RIS for sleep induction [15, 56, 63].

\section{Sleep Is Required to Survive Larval Starvation-Induced} Arrest

The strong induction of sleep during starvation and arrest through aging pathways suggested that sleep might play a role in surviving food deprivation and development cessation $[18,64]$. Thus, we tested adult lifespan along with survival and recovery from L1 arrest in the presence or absence of food in sleepless mutants. We first measured the lifespan of adult worms in the presence of food and during starvation. In both conditions, there was no consistent and significant difference

(G) Hypothetical working model that integrates literature data with our observations. The numbers of assayed worms (n) are displayed below the boxplots. Sleep duration comparisons with N2 were made using the Mann-Whitney U test and were confirmed with Benjamini-Hochberg procedure for multiple comparisons. Error bars indicate the SEM.

See also Figure $\$ 4$ for tissue-specific rescue experiments. 


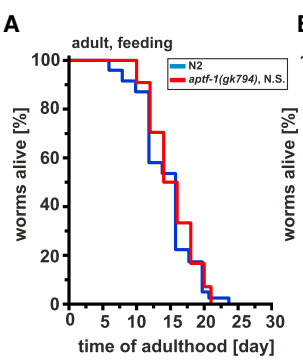

E

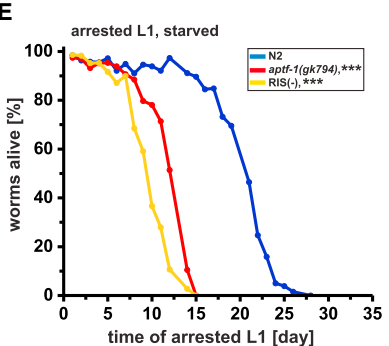

B C

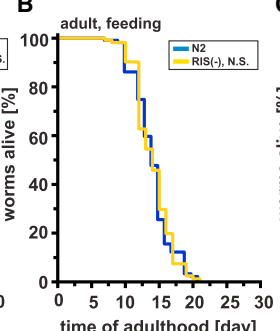

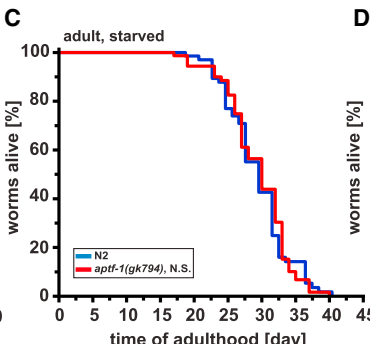

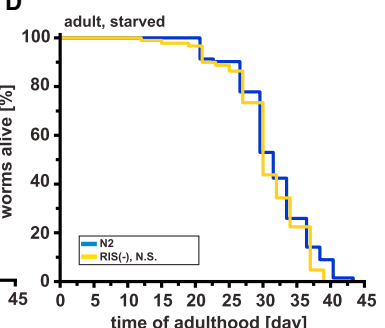

F

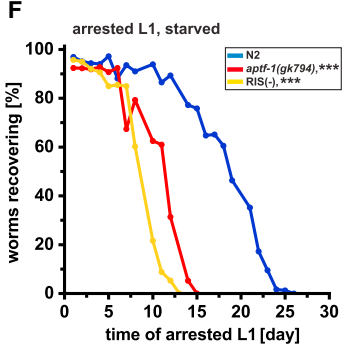

G

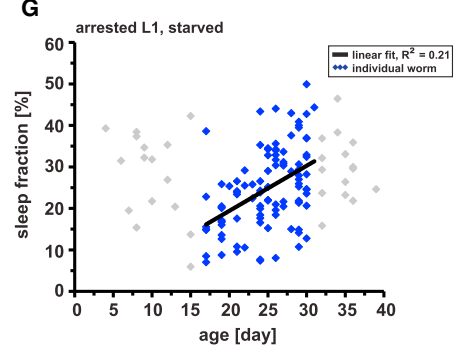

H
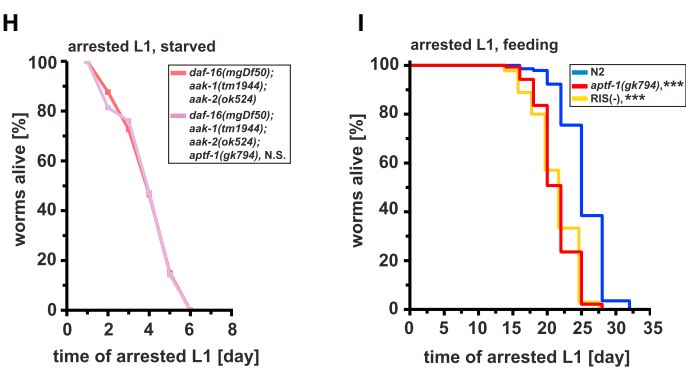

Figure 5. Sleep Is Required for Surviving Larval Starvation-Induced Arrest

Shown are representative experiments; additional replicates can be found in Figure S5.

(A) Adult lifespans of feeding wild-type (blue) and aptf-1(gk794) (red) worms were not significantly different (mean lifespan, N2: 14.6 days, $n=44$ worms; aptf-1(gk794): 15.3 days, $n=43$ worms; $p=0.71$; log rank test).

(B) Lifespan of feeding wild-type (blue) and RIS(-) (yellow) adult worms shows no significant difference (mean lifespan, N2: 14.2 days, $n=94$ worms; RIS(-): 14.1 days, $n=86$ worms; $p=0.71 ; \log$ rank test).

(C and D) No significant difference of lifespan was detected in (C) starved adult aptf-1(gk794) compared to starved N2 (mean lifespan, N2: 29.8 days, $n=60$ worms; aptf-1(gk794): 29.5 days, $n=63$ worms; $p=0.65$; log rank test) and (D) also not in starved RIS(-) (mean lifespan N2: 32.0 days, $n=85$ worms; RIS(-): 30.6 days, $\mathrm{n}=84$ worms; $\mathrm{p}=0.06$; $\log$ rank test).

(E) L1-arrested aptf-1(gk794) mutants and RIS ablated worms showed substantially reduced survival in the absence of food (time at which $50 \%$ of worms were still alive, N2: 20.7 days, $n>60$ worms for each time point; aptf-1(gk794): 12.1 days, $n>60$ worms; ${ }^{* \star *} p<0.001$; RIS(-): 9.4 days, $n>60$ worms; ${ }^{* \star *} p<0.001$; significance shown from day 8; Fischer's exact test).

(F) Arrested L1 aptf-1(gk794) and RIS-ablated worms showed a significant decline in the ability to re-enter development when fed (time at which $50 \%$ of worms were still able to recover, N2: 18.7 days, $n>60$ worms; aptf-1(gk794): 11.4 days, $n>60$ worms; ${ }^{* * *} p<0.001$; RIS(-): 8.5 days, $n>60$ worms; *** $p<0.001$; significance shown from day 8 for RIS(-) and day 10 for aptf-1 mutant; Fischer's exact test).

(G) Sleep amount predicts starvation survival. Arrested starved L1 larvae were cultured in microchambers, and their sleep and survival were quantified. Data from individual worms within the range used for linear fitting (black line) are plotted as blue diamonds (data outside the range of the SD of the mean, including earlydying immobile individuals, were excluded; gray); $R^{2}=0.21$. 
in lifespan between wild-type, aptf-1(-), and RIS-ablated worms (Figures 5A-5D and S5A-S5D). We then tested L1 arrest survival and recoverability. For this, we measured both the time that L1 larvae stayed alive in the absence of food as well as the time until larvae were still able to recover from the arrest and to resume their development when fed [65]. Both survival time and the ability to recover were reduced consistently and significantly by about half in sleepless mutants (Figures $5 \mathrm{E}$. 5F, and $\mathrm{S} 5 \mathrm{E}-\mathrm{S} 5 \mathrm{H})$. Thus, although there does not seem to be a strict requirement of sleep for survival in adult $C$. elegans, it becomes essential in the arrested larva.

To test whether sleep is a predictor of survival, we quantified quiescence for individual starved L1 larvae inside microfluidic devices and also measured their survival span. A correlation was seen between the amount of sleep during the first 2-4 days of starvation and survival (an increase of $1 \%$ sleeping time corresponded to an increase in survival extension by roughly $5 \%$; Figure $5 \mathrm{G}$ ), suggesting that sleep is a predictor for surviving starvation.

If sleep is part of the protective program induced by AAK-1/2 and IIS, then ablating sleep in AAK-1/2 and IIS mutants should not further decrease survival during larval starvation. To test this idea, we looked at survival of aptf-1(-) mutant worms in a FoxO/AAK-1/2 triple mutant background. There was no difference in survival due to sleep loss when we compared the daf-16(mgDf50)/aak-1(tm1944)/aak-2(ok524) triple mutant with daf-16(mgDf50)/aak-1(tm1944)/aak-2(ok524)/aptf-1(gk794) quadruple mutant (Figures $5 \mathrm{H}$ and $\mathrm{S} 5 \mathrm{I}$ ). This is consistent with the view that sleep and AAK/FoxO act in the same pathway.

If the role of sleep were to conserve energy or nutrients, then feeding would suppress the requirement of sleep for survival. To test this idea, we arrested L1 larvae using 5-fluoro-2'-deoxyuridine (FUdR), kept them in the presence of ad libitum E. coli as food source, and measured survival. The arrested larvae consumed the bacteria (Figures S6A and S6B) and showed sleeping behavior (Figure S6C). However, sleepless worms still had a significantly shorter lifetime than the sleeping control (Figures $5 \mathrm{I}$ and $\mathrm{S} 5 \mathrm{~J}$ ). This suggests that, although resource preservation is important, sleep acts beyond energy or nutrient conservation to achieve survival.

Sleep Counteracts the Progression of Aging Phenotypes during Starvation-Induced Arrest

Recent work demonstrated that L1 arrested larvae show a progression of aging phenotypes leading to death. Thus, the cause of death of arrested larvae is similar to the one in the adult [65]. We wondered whether sleep is required for survival by slowing down the progression of aging phenotypes during starvation. To test this idea, we looked at established markers of aging, such as the deterioration of body wall muscle fibers, mitochondrial fragmentation, and protein aggregation [65]. We crossed aptf-1(-) into transgenic strains in which either muscle myosin, muscle mitochondria, or an aggregation-prone polyglutamine containing protein, poly-Q35::YFP, were fluorescently labeled [65-69]. L1 larvae were starved, and the progression of aging was monitored by fluorescence microscopy until the animals had died. The morphology of the aging markers looked normal in freshly starved L1 aptf-1(-). However, as time passed, aging markers progressed more quickly in the sleepless mutant compared with the wild-type. Muscle fibers degraded more quickly, mitochondria underwent fission earlier, and protein ag gregation occurred faster. Sleepless worms reached the same maximal level of aging marker progression approximately ten days earlier compared with the wild-type (Figure 6). Together, these results suggest that, rather than merely saving energy, sleep is required for survival of starvation by counteracting the progression of aging processes.

\section{DISCUSSION}

To understand why sleep is important for $C$. elegans, we monitored behavioral activity and RIS calcium transients to survey the prevalence of sleep across life stages and physiological conditions. We tested for the effects of food quality and availability, as these define the major physiological conditions in the life of $C$. elegans, which proliferates in ephemeral microbe-rich habitats and is able to survive long periods of starvation [19]. Sleep occurred during most stages and conditions that were analyzed. RIS depolarized at the onset of sleep bouts and typically showed activity until the end of the sleep bout, inversely mirroring the pattern of behavioral activity. This pattern of RIS depolarization is consistent with the view that RIS is the inducer of sleep, and the maintenance of high RIS activity throughout most of the sleep bout suggests that sleep is under constant control of RIS. During longer sleep bouts, which were, for example, observed in dauer larvae and daf-2(-), calcium transients still correlated with sleep onset but decreased already before the end of the bout. This may speculatively be caused by an additional mechanism that may dampen wakefulness independently of RIS, thus potentially explaining a reduced requirement for RIS activity for maintaining sleep. Consistent with this speculation, spontaneous motion is reduced in daf-2(-) and dauer larvae [70]. daf-2(-) mutants showed a residual fraction of immobility that could not be suppressed by aptf-1(-). Similarly, a small fraction of residual quiescence was observed in the absence of functional RIS when worms were fed with bacteria in the presence of growth medium. Different scenarios could explain this residual quiescence. First, occasionally, slow locomotion may be falsely scored as quiescence by the analysis algorithm. Second, quiescence may exist that is not sleep or is sleep but is not induced by RIS. Severe cellular stress can also induce behavioral quiescence independently of RIS, involving activation of the ALA neuron $[39,41]$. Thus, hypothetically, some of the conditions that we

(H) Starved arrested L1 aak-1(tm1944)/aak-2(ok524)/daf-16(mgDf50)/aptf-1(gk794) quadruple mutants showed no reduced survival compared to aak-1(tm1944)/ aak-2(ok524)/daf-16(mgDf50) triple mutants (time at which 50\% of worms were still alive, aak-1/(tm1944)/aak-2(ok524)/daf-16(mgDf50): 3.9 days, $n>60$ worms; aak-1(tm1944)/aak-2(ok524)/daf-16(mgDf50)/aptf-1(gk794): 3.9 days, $n>60$ worms; $p>0.05$ for all days; Fischer's exact test).

(I) FUdR-arrested L1s in the presence of food have a decreased survival. Arrested L1 survival is significantly decreased in aptf-1(gk794) and in RIS(-) worms (mean survival, N2: 25.7 days, $n=143$ worms; aptf-1 (gk794): 21.9 days, $n=140$ worms; ;** $\mathrm{p}<0.001 ; \mathrm{RIS}(-)$ : 22.1 days, $\mathrm{n}=135$ worms; ${ }^{* * *} \mathrm{p}<0.001 ;$ log rank test). See also Figures S5 and S6 and Tables S1, S2, S3, S4, and S5 for additional replicates and details on food consumption. 
A

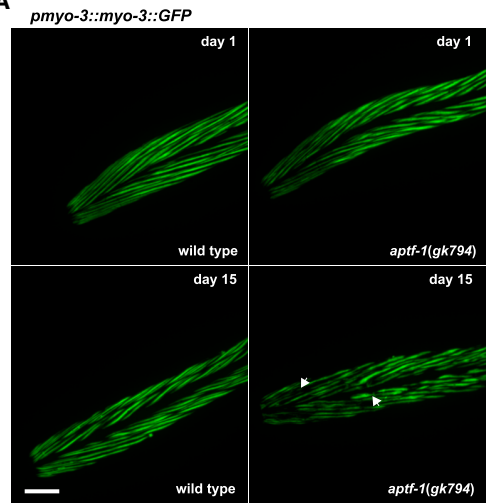

C pmyo-3::GFP(NLS), myo-3::mitoGFP
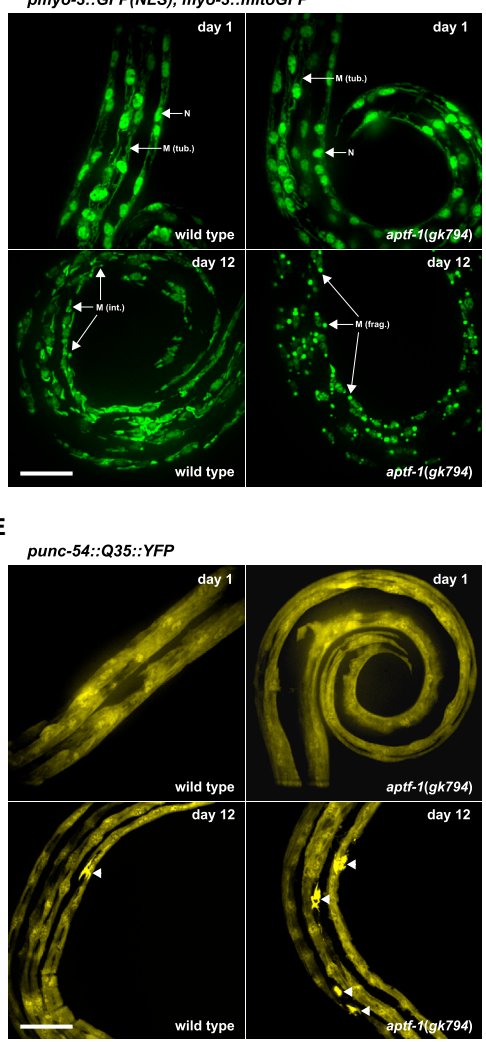

B

Tiber disruption in the head of arrested L1

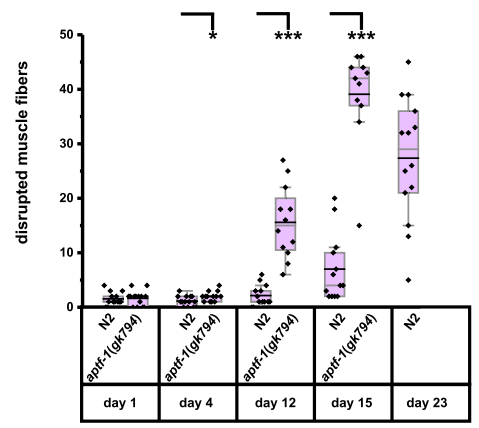

D

fragmentation of muscular mitochondria of arrested L1

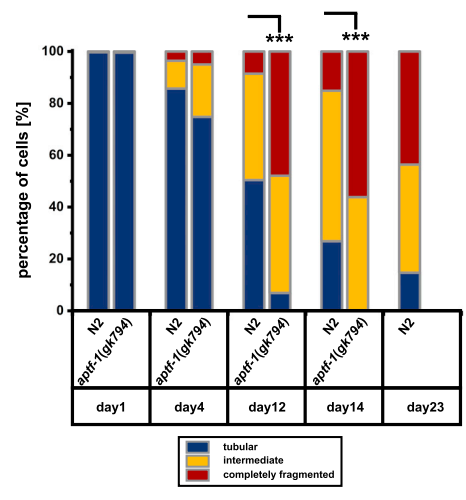

F aggregation of poly-Q35 in muscle cell of arrested L1

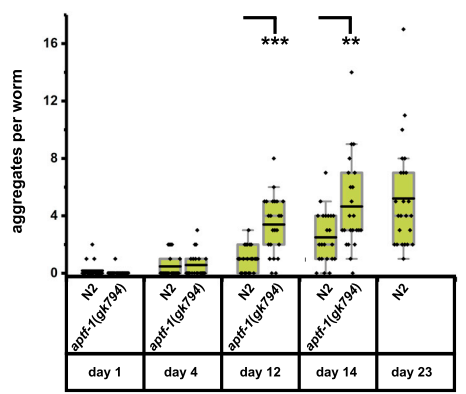

3620 Current Biology 28, 3610-3624, November 19, 2018 
investigated may have also triggered a stress response and RIS-independent quiescence. Third, in some instances, the genetic RIS ablation might not have been fully penetrant due to transgene silencing or mutant suppression. Because, overall, only a minority of quiescent counts did not depend on RIS, we did not further investigate their cause. In summary, it emerges that, under physiological conditions, the majority of behavioral quiescence in $C$. elegans presents sleep caused by RIS.

In C. elegans, sleep amount strongly depends on food quality and availability, with starvation, developmental arrest, and diapause presenting the strongest sleep triggers. We hence focused our analysis on the role of sleep during starvation conditions. Starvation and aging are intimately linked: seminal work from $C$. elegans has led to the identification of genes controlling aging, including FoxO and AMP kinase, and these healthy aging genes are also required for surviving starvation [20, 21]. Genetic analysis in mice and humans showed that these genes are conserved regulators of aging [21]. Here, building on previous work on aging and sleep in C. elegans [11, 36, 51, 52], we linked the network of known longevity genes to sleep induction. This longevity network consists of IIS and sirtuin signaling, which control FoxO and AMPK, which activate the RIS neuron and thus induce sleep. FoxO and AMPK can act in tissues outside of RIS (even though AMPK can also act within RIS), suggesting that multiple tissues can signal starvation to induce sleep.

All organisms are threatened with the possibility of experienced famine in their life. Thus, strategies to survive the depletion of food are highly adaptive and underlying mechanisms are deeply conserved. Across species, animals show a stereotyped biphasic behavioral response to starvation. First, arousal is increased and sleep is reduced [12-16]. This phase is then followed by a phase of reduced physical activity [8-11]. Extreme conditions of food deprivation can lead to a quiescence state known as torpor, which is thought to preserve energy by shutting down physical and metabolic activity. Torpor differs from sleep in that the behavioral quiescence is not readily reversible. By contrast, sleep has been proposed to not only conserve energy but also to allocate resources [17, 71].

Does sleep serve different or similar functions during different conditions? What are the identities of these functions? And how are they carried out? Sleep has been proposed to serve diverse roles, ranging from energy conservation and allocation [6, 71] and restoration and regulation of key cellular and metabolic processes $[72,73]$ to higher brain functions $[74,75]$. Viability assays revealed an important and specific role of sleep in surviving starvation-induced developmental arrest. Although sleep is vital for surviving starvation in larvae, we did not find any evidence that it is essential for lifespan extension in adults. Consisten with this finding, juvenile stages typically sleep more and are more susceptible to sleep deprivation also in other species $[23,76,77]$. A major biological difference between starvation in adult and larval worms is that adult worms have already completed development, with all somatic cells being postmitotic, whereas the larva has to arrest their development, which includes the inhibition of cell division [78]. Thus, the survival experiments suggest that the vulnerability of larvae to sleep loss relates to the arrested development. Because adult worms also spent substantial time asleep, this suggests that sleep may also serve additional functions, which may not be essential for survival under ideal laboratory conditions [50,51]. How does sleep prevent death during starvation-induced arrest? The progression of aging phenotypes, but not residual energy reserves, has been shown to predict the ability of larvae to survive and recover from starvation-induced arrest, suggesting that arrested worms do not die because they run out of energy but because of cellular demise that is mechanistically similar to aging [65] Consistent with this finding, we observed that sleep slows down the aging phenotype progression during starvationinduced arrest. Thus, sleep appears to be required for starvation survival, not only because it saves energy from being burned for motility but rather because it counteracts aging progression. Speculatively, sleep might allocate resources away from behavioral activity toward cell-protecting pathways that are similar to anti-aging processes.

Sleep is ancient and most likely evolved to serve basic, essential needs, such as recuperation from stress and illness [41, 79]. Here, we show that sleep during starvation-induced developmental arrest presents an anti-aging program required for survival. This function could have been an important ancient reason for a sleep response, which was co-opted later during evolution to also serve higher brain functions. Most likely, the molecula link between starvation, development, aging, and sleep also plays a role in human sleep. Figuring out how sleep protects against cellular demise will be instructive to understand its revitalizing functions.

Figure 6. Sleep Counteracts the Progression of Aging Phenotypes during L1 Arrest

(A and B) Body-wall muscle myosin deteriorates faster in aptf-1(-). (A) Shown is the head region of transgenic worms expressing GFP-tagged myosin (representative examples). After two days of starvation, muscle structure appears intact in both wild-type and aptf-1(gk794). After fifteen days of starvation, muscle myosin deterioration has progressed much more in aptf-1(gk794) compared to wild-type. Arrowheads point to gaps in muscle structure. (B) Quantification of muscle deterioration. 4 days: $n>10, \mathrm{N2}: 1.1 \pm 0.3$; aptf-1(gk794): $2 \pm 0.3,{ }^{*} \mathrm{p}<0.05 ; 12$ days: $\mathrm{n}>10, \mathrm{~N} 2: 2.2 \pm 0.5$; aptf-1(gk794): $15.6 \pm 1.9,{ }^{* \star *} \mathrm{p}<0.001 ; 15$ days: $\mathrm{n}>10, \mathrm{~N} 2: 7 \pm 1.7 ;$ aptf-1(gk794): $39.1 \pm 2.7, * * * \mathrm{p}<0.001$, two-sample t test.

( $C$ and $D$ ) Mitochondrial fragmentation is increased in aptf-1(-). (C) Mitochondrial morphology appears normal in one day starved larvae, but the initially tubular mitochondria fragmented much faster in aptf-1(-) compared with the wild-type. Mitochondria in body wall muscles were labeled using GFP and were tubular mitochond classified as tubular ( $\mathrm{M}$ [tub.]), intermediately fragmented (M [int.]), or completely fragmented (M. [frag.]). Shown are the mid-body regions of worms, and arrows point to example mitochondrial structures or nucleus $(\mathrm{N})$. (D) For the quantification of mitochondrial deterioration, the percentage of muscle cells displaying each level of fragmentation was determined (12 days $-\mathrm{M}$ (frag.): $n>20$, N2: 8.5\% $\pm 3.3 \%$; aptf-1(gk794): $47.9 \% \pm 5.9 \%,{ }^{* \star *} p<0.001 ; 14$ days $-M$ (frag.): $n>20, \mathrm{~N} 2: 15 \% \pm 3.4 \%$; aptt-1(gk794): $56.1 \% \pm 6.1 \%,{ }^{* * *} \mathrm{p}<0.001$, two-sample $t$ test).

( $E$ and F) Protein aggregation is increased in aptf-1(-). (E) Poly-Q35 was expressed in muscle, and the number of aggregates formed was scored. In one day starved L1, there were almost no detectable aggregates in both wild-type and aptf-1 (gk794). Shown are the mid-body regions of worms with arrowheads pointing at aggregates. (F) The number of aggregates increased much faster in aptf-1(gk794) compared with wild-type (12 days: $n>20, \mathrm{N2}: 1 \pm 0.2$; aptf-1 (gk794): $3.4 \pm$ $0.4,{ }^{* * *} \mathrm{p}<0.001 ; 14$ days: $\mathrm{n}>20, \mathrm{~N} 2: 2.5 \pm 0.4 ;$ aptf-1 (gk794): $4.7 \pm 0.7,{ }^{* *} \mathrm{p}<0.01$, two-sample t test). 


\section{STAR $\star M E T H O D S$}

Detailed methods are provided in the online version of this paper and include the following:

- KEY RESOURCES TABLE

- CONTACT FOR REAGENT AND RESOURCE SHARING

- EXPERIMENTAL MODEL AND SUBJECT DETAILS

$\bigcirc$ Worm maintenance and strains

- METHOD DETAILS

- Molecular biology and transgenic strain generation

- Dauer pheromone extraction and dauer larvae generation

Long-term imaging

Optogenetics and light stimulation

- Image Analysis

Lifespan and survival assays

Quantification of bacteria consumption

Quantification of aging markers

- QUANTIFICATION AND STATISTICAL ANALYSIS

\section{SUPPLEMENTAL INFORMATION}

Supplemental Information includes six figures and seven tables and can be found with this article online at https://doi.org/10.1016/j.cub.2018.10.009.

\section{ACKNOWLEDGMENTS}

We thank Sabine König for extracting dauer pheromone; Juliane Schwarz for creating the pmec-4::ReaChr and psra-6::ReaChr strains; Michal Turek for creating the pflp-11::GCaMP and pflp-11::ArchT strains; Jan Konietzk for providing the agarose microchamber with N2-arrested L 1 worms; Rict Roy, Shoolin Li, and Masamitsu Fukuyam for providing rescue straichard Roy, Shaoli Li, and Masamilou Fukuyama for providing rescue strains; an Alexander Sibersdort (Centre for Statistics of the University of Göttingen (a) is funded by NHH Offec Research Intrastructure Programs (P40 OD010440). This work was supported by an Excellence Stipend of the Göttingen Graduate School for Neurosciences, Biophysics, and Molecular Biosciences to F.M., the Max Planck Society (Max Planck Research Group) to H.B., a European Research Council Starting Grant under the European Union's Horizon 2020 research and innovation program (ID: 637860; SLEEPCONTROL) to H.B. and an Exploration Grant of the Boehringer Ingelheim Stiftung Germany (Effects of sleep on ageing using $C$. elegans as a model system) to H.B. Additional data can be found in the Supplemental Information.

\section{AUTHOR CONTRIBUTIONS}

Y.W., F.M., and J.P. designed, performed, and analyzed experiments. H.B. conceived the general outline of the project, supervised the work, and wrote the paper.

\section{DECLARATION OF INTERESTS}

The authors declare no competing interests.

Received: February 20, 2018

Revised: September 11, 2018

Revised. September 11,2018

Accepted: October 2, 2018

\section{REFERENCES}

1. Cirelli, C., and Tononi, G. (2008). Is sleep essential? PLoS Biol. 6, e216.

2. Colten, H.R., and Altevogt, B.M. (2006). Functional and economic impac

of sleep loss and sleep-related disorders. In Sleep Disorders and Sleep
Deprivation: An Unmet Public Health Problem, H.R. Colten, and B.M. Altevogt, eds. (National Academies Press), pp. 137-172.

3. Joiner, W.J. (2016). Unraveling the evolutionary determinants of sleep. Curr. Biol. 26, R1073-R1087.

4. Nath, R.D., Bedbrook, C.N., Abrams, M.J., Basinger, T., Bois, J.S., Prober, D.A., Sternberg, P.W., Gradinaru, V., and Goentoro, L. (2017). The jellyfish Cassiopea exhibits a sleep-like state. Curr. Biol. 27, 2984-2990.e3.

5. Rechtschaffen, A., and Bergmann, B.M. (1995). Sleep deprivation in the rat by the disk-over-water method. Behav. Brain Res, 69, 55-63.

6. Siegel, J.M. (2009). Sleep viewed as a state of adaptive inactivity. Nat. Rev. Neurosci. 10, 747-753.

7. Lesku, J.A., Rattenborg, N.C., Valcu, M., Vyssotski, A.L., Kuhn, S., Kuemmeth, F., Heidrich, W., and Kempenaers, B. (2012). Adaptive sleep loss in polygynous pectoral sandpipers. Science 337, 1654-1658.

8. Novak, C.M., Jiang, X., Wang, C., Teske, J.A., Kotz, C.M., and Levine, J.A. (2005). Caloric restriction and physical activity in zebrafish (Danio rerio). Neurosci. Lett. 383, 99-104.

9. Severinsen, T., and Munch, I.C. (1999). Body core temperature during food restriction in rats. Acta Physiol. Scand. 165, 299-305.

10. McCloskey, R.J., Fouad, A.D., Churgin, M.A., and Fang-Yen, C. (2017). Food responsiveness regulates episodic behavioral states in Caenorhabditis elegans. J. Neurophysiol. 117, 1911-1934.

11. Skora, S., Mende, F., and Zimmer, M. (2018). Energy scarcity promotes a brain-wide sleep state modulated by insulin signaling in C. elegans. Cell Rep. 22, 953-966.

12. Danguir, J., and Nicolaidis, S. (1979). Dependence of sleep on nutrients' availability. Physiol. Behav. 22, 735-740.

13. Borbély, A.A. (1977). Sleep in the rat during food deprivation and subsequent restitution of food. Brain Res. 124, 457-471.

14. Wang, T., Hung, C.C., and Randall, D.J. (2006). The comparative physiology of food deprivation: from feast to famine. Annu. Rev. Physiol. 68, 223-251.

15. Ahmadi, M., and Roy, R. (2016). AMPK acts as a molecular trigger to coordinate glutamatergic signals and adaptive behaviours during acute starvation. eLife 5, e16349.

16. Goetting, D.L., Soto, R., and Van Buskirk, C. (2018). Food-dependent plasticity in Caenorhabditis elegans stress-induced sleep is mediated by TOR-FOXA and TGF- $\beta$ signaling. Genetics 209, 1183-1195.

17. Kilduff, T.S., Krilowicz, B., Milsom, W.K. Trachsel, L, and Wang, L.C. (1993). Sleep and mammalian hibernation: homologous adaptations and homologous processes? Sleep 16, 372-386.

18. Kapahi, P., Kaeberlein, M., and Hansen, M. (2017). Dietary restriction and lifespan: lessons from invertebrate models. Ageing Res. Rev. 39, 3-14.

19. Frézal, L., and Félix, M.A. (2015). C. elegans outside the Petri dish. eLife 4, e05849.

20. Baugh, L.R. (2013). To grow or not to grow: nutritional control of development during Caenorhabditis elegans L1 arrest. Genetics 194, 539-555.

21. Kenyon, C.J. (2010). The genetics of ageing. Nature 464, 504-512.

22. Trojanowski, N.F., and Raizen, D.M. (2016). Call it worm sleep. Trends Neurosci. 39, 54-62.

23. Kayser, M.S., and Biron, D. (2016). Sleep and development in genetically tractable model organisms. Genetics 203, 21-33.

24. Saper, C.B., Scammell, T.E., and Lu, J. (2005). Hypothalamic regulation of sleep and circadian rhythms. Nature 437, 1257-1263.

25. Bringmann, H. (2018). Sleep-active neurons: conserved motors of sleep. Genetics 208, 1279-1289.

26. Saper, C.B., Fuller, P.M., Pedersen, N.P., Lu, J., and Scammell, T.E. (2010). Sleep state switching. Neuron 68, 1023-1042.

27. Alam, M.A., Kumar, S., McGinty, D., Alam, M.N., and Szymusiak, R. (2014). Neuronal activity in the preoptic hypothalamus during sleep deprivation and recovery sleep. J. Neurophysiol. 111,287-299.

3622 Current Biology 28, 3610-3624, November 19, 2018 
28. Pimentel, D., Donlea, J.M., Talbot, C.B., Song, S.M., Thurston, A.J.F., and Miesenböck, G. (2016). Operation of a homeostatic sleep switch. Nature 536, 333-337.

29. Turek, M., Lewandrowski, I., and Bringmann, H. (2013). An AP2 transcription factor is required for a sleep-active neuron to induce sleep-like quiescence in C. elegans. Curr. Biol. 23, 2215-2223.

30. Turek, M., Besseling, J., Spies, J.P., König, S., and Bringmann, H. (2016). Sleep-active neuron specification and sleep induction require FLP-11 neuropeptides to systemically induce sleep. eLife 5, e12499.

31. Spies, J., and Bringmann, H. (2018). Automated detection and manipulation of sleep in C. elegans reveals depolarization of a sleep-active neuron tion of sleep in C. elegans reveals depolarization of a sleep-active neuron during
9732.

32. You, Y.J., Kim, J., Raizen, D.M., and Avery, L. (2008). Insulin, cGMP, and TGF-beta signals regulate food intake and quiescence in C. elegans: a model for satiety. Cell Metab. 7, 249-257.

33. Cassada, R.C., and Russell, R.L. (1975). The dauerlarva, a post-embryonic developmental variant of the nematode Caenorhabditis elegans. Dev. Biol. 46, 326-342.

34. Bringmann, H. (2011). Agarose hydrogel microcompartments for imaging sleep- and wake-like behavior and nervous system development in Caenorhabditis elegans larvae. J. Neurosci. Methods 201, 78-88.

35. Turek, M., Besseling, J., and Bringmann, H. (2015). Agarose microchambers for long-term calcium imaging of Caenorhabditis elegans. J. Vis. Exp. e52742.

36. Gaglia, M.M., and Kenyon, C. (2009). Stimulation of movement in a quiescent, hibernation-like form of Caenorhabditis elegans by dopamine signaling. J. Neurosci. 29, 7302-7314.

37. Hu, P.J. (2007). Dauer. WormBook, 1-19.

38. Nichols, A.L.A., Eichler, T., Latham, R., and Zimmer, M. (2017). A global brain state underlies C. elegans sleep behavior. Science 356, eaam6851.

39. Trojanowski, N.F., Nelson, M.D., Flavell, S.W., Fang-Yen, C., and Raizen, D.M. (2015). Distinct mechanisms underlie quiescence during two Caenorhabditis elegans sleep-like states. J. Neurosci. 35, 14571-14584.

40. Van Buskirk, C., and Sternberg, P.W. (2007). Epidermal growth factor signaling induces behavioral quiescence in Caenorhabditis elegans. Nat. Neurosci. 10, 1300-1307.

41. Hill, A.J., Mansfield, R., Lopez, J.M., Raizen, D.M., and Van Buskirk, C. (2014). Cellular stress induces a protective sleep-like state in C. elegans. Curr. Biol. 24, 2399-2405.

42. Raizen, D.M., Zimmerman, J.E., Maycock, M.H., Ta, U.D., You, Y.J., Sundaram, M.V., and Pack, A.I. (2008). Lethargus is a Caenorhabditis elegans sleep-like state. Nature 451, 569-572.

43. Iwanir, S., Tramm, N., Nagy, S., Wright, C., Ish, D., and Biron, D. (2013). The microarchitecture of $\mathrm{C}$. elegans behavior during lethargus: homeostatic bout dynamics, a typical body posture, and regulation by a central neuron. Sleep 36, 385-395.

44. Campbell, S.S., and Tobler, I. (1984). Animal sleep: a review of sleep duration across phylogeny. Neurosci. Biobehav. Rev. 8, 269-300.

45. Edwards, S.L., Charlie, N.K., Milfort, M.C., Brown, B.S., Gravlin, C.N., Knecht, J.E., and Miller, K.G. (2008). A novel molecular solution for ultraviolet light detection in Caenorhabditis elegans. PLoS Biol. 6, e198.

46. Lin, J.Y., Knutsen, P.M., Muller, A., Kleinfeld, D., and Tsien, R.Y. (2013). ReaChR: a red-shifted variant of channelrhodopsin enables deep transcranial optogenetic excitation. Nat. Neurosci. 16, 1499-1508.

47. Ward, A., Liu, J., Feng, Z., and Xu, X.Z. (2008). Light-sensitive neurons and channels mediate phototaxis in C. elegans. Nat. Neurosci. 11, 916-922.

48. Han, X., Chow, B.Y., Zhou, H., Klapoetke, N.C., Chuong, A., Rajimehr, R., Yang, A., Baratta, M.V., Winkle, J., Desimone, R., and Boyden, E.S. (2011). A high-light sensitivity optical neural silencer: development and application to optogenetic control of non-human primate cortex. Front. Syst. Neurosci. 5, 18.
49. Schwarz, J., Lewandrowski, I., and Bringmann, H. (2011). Reduced activity of a sensory neuron during a sleep-like state in Caenorhabditis elegans. Curr. Biol. 21, R983-R984.

50. Sanders, J., Scholz, M., Merutka, I., and Biron, D. (2017). Distinct unfolded protein responses mitigate or mediate effects of nonlethal deprivation of C. elegans sleep in different tissues. BMC Biol. 15, 67.

51. Driver, R.J., Lamb, A.L., Wyner, A.J., and Raizen, D.M. (2013). DAF-16 FOXO regulates homeostasis of essential sleep-like behavior during larva transitions in C. elegans. Curr. Biol. 23, 501-506.

52. Nagy, S., Tramm, N., Sanders, J., Iwanir, S., Shirley, I.A., Levine, E., and Biron, D. (2014). Homeostasis in C. elegans sleep is characterized by two behaviorally and genetically distinct mechanisms. eLife 3, e04380.

53. Baugh, L.R., and Sternberg, P.W. (2006). DAF-16/FOXO regulates transcription of $\mathrm{cki}-1 / \mathrm{Cip} / \mathrm{Kip}$ and repression of lin-4 during C. elegans L1 arrest. Curr. Biol. 16, 780-785.

54. Paradis, S., and Ruvkun, G. (1998). Caenorhabditis elegans Akt/PKB transduces insulin receptor-like signals from AGE-1 PI3 kinase to the DAF-16 transcription factor. Genes Dev. 12, 2488-2498.

55. Hardie, D.G. (2011). AMP-activated protein kinase: an energy sensor that regulates all aspects of cell function. Genes Dev. 25, 1895-1908.

56. Fukuyama, M., Sakuma, K., Park, R., Kasuga, H., Nagaya, R., Atsumi, Y., Shimomura, Y., Takahashi, S., Kajiho, H., Rougvie, A., et al. (2012). C. elegans AMPKs promote survival and arrest germline development during nutrient stress. Biol. Open 1, 929-936.

57. Berdichevsky, A., Viswanathan, M., Horvitz, H.R., and Guarente, L. (2006). C. elegans SIR-2.1 interacts with 14-3-3 proteins to activate DAF-16 and extend life span. Cell 125, 1165-1177.

58. Curtis, R., O'Connor, G., and DiStefano, P.S. (2006). Aging networks in Caenorhabditis elegans: AMP-activated protein kinase (aak-2) links multiple aging and metabolism pathways. Aging Cell 5, 119-126.

59. Lin, K., Hsin, H., Libina, N., and Kenyon, C. (2001). Regulation of the Caenorhabditis elegans longevity protein DAF-16 by insulin/IGF-1 and germline signaling. Nat. Genet. 28, 139-145.

60. Libina, N., Berman, J.R., and Kenyon, C. (2003). Tissue-specific activities of C. elegans DAF-16 in the regulation of lifespan. Cell 115, 489-502.

61. Ehlen, J.C., Brager, A.J., Baggs, J., Pinckney, L., Gray, C.L., DeBruyne, J.P. Esser, K.A., Takahashi, J.S., and Paul, K.N. (2017). Bmal1 function in skeletal muscle regulates sleep. eLife 6 , e26557.

62. Laranjeiro, R., Harinath, G., Burke, D., Braeckman, B.P., and Driscoll, M. (2017). Single swim sessions in C. elegans induce key features of mammalian exercise. BMC Biol. 15, 30.

63. Narbonne, P., and Roy, R. (2009). Caenorhabditis elegans dauers need LKB1/AMPK to ration lipid reserves and ensure long-term survival. Nature 457, 210-214

64. Kaeberlein, T.L., Smith, E.D., Tsuchiya, M., Welton, K.L., Thomas, J.H., Fields, S., Kennedy, B.K., and Kaeberlein, M. (2006). Lifespan extension in Caenorhabditis elegans by complete removal of food. Aging Cell 5 , 487-494.

65. Roux, A.E., Langhans, K., Huynh, W., and Kenyon, C. (2016). Reversible age-related phenotypes induced during larval quiescence in C. elegans. Cell Metab. 23, 1113-1126.

66. Yasuda, K., Ishii, T. Suda, H., Akatsuka, A., Hartman, P.S., Goto, S., Miyazawa, M., and Ishii, N. (2006). Age-related changes of mitochondria structure and function in Caenorhabditis elegans. Mech. Ageing Dev. 127, 763-770.

67. Morley, J.F., Brignull, H.R., Weyers, J.J., and Morimoto, R.I. (2002). The threshold for polyglutamine-expansion protein aggregation and cellular toxicity is dynamic and influenced by aging in Caenorhabditis elegans. Proc. Natl. Acad. Sci. USA 99, 10417-10422.

68. David, D.C., Ollikainen, N., Trinidad, J.C., Cary, M.P., Burlingame, A.L, and Kenyon, C. (2010). Widespread protein aggregation as an inherent part of aging in C. elegans. PLoS Biol. 8, e1000450.

69. Campagnola, P.J., Millard, A.C., Terasaki, M., Hoppe, P.E., Malone, C.J., and Mohler, W.A. (2002). Three-dimensional high-resolution second-

Current Biology 28, 3610-3624, November 19, 20183623 
harmonic generation imaging of endogenous structural proteins in biolog ical tissues. Biophys. J. 82, 493-508.

70. Gems, D., Sutton, A.J., Sundermeyer, M.L., Albert, P.S., King, K.V., Edgley, M. L., Larsen, P.L., and Riddle, D.L. (1998). Two pleiotropic classes of daf-2 mutation affect larval arrest, adult behavior, repro

71. Schmidt, M.H. (2014). The energy allocation function of sleep: a unifying theory of sleep, torpor, and continuous wakefulness. Neurosci. Biobehav. Rev. 47, 122-153.

72. Mackiewicz, M., Shockley, K.R., Romer, M.A., Galante, R.J., Zimmerman, J.E., Naidoo, N., Baldwin, D.A., Jensen, S.T., Churchill, G.A., and Pack, A.I. (2007). Macromolecule biosynthesis: a key function of sleep. Physiol. Genomics 31, 441-457.

73. Tu, B.P., and McKnight, S.L. (2006). Metabolic cycles as an underlying basis of biological oscillations. Nat. Rev. Mol. Cell Biol. 7, 696-701.

74. Diekelmann, S., and Born, J. (2010). The memory function of sleep. Nat. Rev. Neurosci. 11, 114-126.

75. Krause, A.J., Simon, E.B., Mander, B.A., Greer, S.M., Saletin, J.M. Goldstein-Piekarski, A.N., and Walker, M.P. (2017). The sleep-deprived human brain. Nat. Rev. Neurosci. 18, 404-418.

76. Roffwarg, H.P., Muzio, J.N., and Dement, W.C. (1966). Ontogenetic development of the human sleep-dream cycle. Science 152, 604-619.

77. Kayser, M.S., Yue, Z., and Sehgal, A. (2014). A critical period of sleep for development of courtship circuitry and behavior in Drosophila. Science 344, 269-274.

78. Sulston, J.E., and Horvitz, H.R. (1977). Post-embryonic cell lineages of the nematode, Caenorhabditis elegans. Dev. Biol. 56, 110-156.

79. Krueger, J.M., Frank, M.G., Wisor, J.P., and Roy, S. (2016). Sleep function: toward elucidating an enigma. Sleep Med. Rev. 28, 46-54.

80. Labrousse, A., Chauvet, S., Couillault, C., Kurz, C.L., and Ewbank, J.J. (2000). Caenorhabditis elegans is a model host for Salmonella typhimurium. Curr. Biol. 10, 1543-1545.

81. Nguyen, J.P., Linder, A.N., Plummer, G.S., Shaevitz, J.W., and Leifer, A.M (2017). Automatically tracking neurons in a moving and deforming brain. PLoS Comput. Biol. 13, e1005517.

82. Mair, W., Morantte, I., Rodrigues, A.P., Manning, G., Montminy, M., Shaw, R.J., and Dillin, A. (2011). Lifespan extension induced by AMPK and calcineurin is mediated by CRTC-1 and CREB. Nature 470, 404-408.

83. Fire, A., Xu, S., Montgomery, M.K., Kostas, S.A., Driver, S.E., and Mello, C.C. (1998). Potent and specific genetic interference by double-stranded RNA in Caenorhabditis elegans. Nature 391, 806-811.
84. Kramer, J.M., and Johnson, J.J. (1993). Analysis of mutations in the sqt-1 and rol-6 collagen genes of Caenorhabditis elegans. Genetics 135, 10351045.

85. Zhang, P., Judy, M., Lee, S.J., and Kenyon, C. (2013). Direct and indirect gene regulation by a life-extending FOXO protein in $\mathrm{C}$. elegans: roles for GATA factors and lipid gene regulators. Cell Metab. 17, 85-100.

86. Urmersbach, B., Besseling, J., Spies, J.P., and Bringmann, H. (2016). Automated analysis of sleep control via a single neuron active at sleep onset in C. elegans. Genesis 54, 212-219.

87. Brenner, S. (1974). The genetics of Caenorhabditis elegans. Genetics 77, 71-94.

88. Ahringer, A. (2006). Reverse genetics. WormBook 1-43.

89. Merritt, C., and Seydoux, G. (2010). Transgenic solutions for the germline. WormBook, 1-21.

90. Redemann, S., Schloissnig, S., Ernst, S., Pozniakowsky, A., Ayloo, S., Hyman, A.A., and Bringmann, H. (2011). Codon adaptation-based control of protein expression in C. elegans. Nat. Methods 8, 250-252.

91. Wilm, T., Demel, P., Koop, H.U., Schnabel, H., and Schnabel, R. (1999). Ballistic transformation of Caenorhabditis elegans. Gene 229, 31-35.

92. Praitis, V., Casey, E., Collar, D., and Austin, J. (2001). Creation of low-copy integrated transgenic lines in Caenorhabditis elegans. Genetics 157 1217-1226.

93. Golden, J.W., and Riddle, D.L. (1984). The Caenorhabditis elegans dauer larva: developmental effects of pheromone, food, and temperature. Dev. Biol. 102, 368-378.

94. Stiernagle, T. (2006). Maintenance of C. elegans. WormBook, 1-11.

95. Nagy, S., Raizen, D.M., and Biron, D. (2014). Measurements of behavioral quiescence in Caenorhabditis elegans. Methods 68, 500-507.

96. Lewis, J.A., and Fleming, J.T. (1995). Basic culture methods. Methods Cell Biol. 48, 3-29.

97. Jung, J., Nakajima, M., Kojima, M., Ooe, K., and Fukuda, T. (2012). Microchip device for measurement of body volume of $C$. elegans as bioindicator application. J. Micro-Nano Mech. 7, 3-11.

98. Herndon, L.A., Schmeissner, P.J., Dudaronek, J.M., Brown, P.A., Listner, K.M., Sakano, Y., Paupard, M.C., Hall, D.H., and Driscoll, M. (2002). Stochastic and genetic factors influence tissue-specific decline in ageing C. elegans. Nature $419,808-814$. 


\section{STAR $\star$ METHODS}

\section{KEY RESOURCES TABLE}

\begin{tabular}{|c|c|c|}
\hline REAGENT or RESOURCE & SOURCE & IDENTIFIER \\
\hline \multicolumn{3}{|l|}{ Bacterial and Virus Strains } \\
\hline OP50 Escherichia coli B, Uracil auxotroph & Caenorhabditis Genetics Center & OP50 \\
\hline OP50 Escherichia coli that contains a GFP plasmid (pFPV25.1) & Caenorhabditis Genetics Center [80] & OP50-GFP \\
\hline \multicolumn{3}{|l|}{ Chemicals, Peptides, and Recombinant Proteins } \\
\hline FUDR, 5-Fluoro-2'-deoxyuridine & Sigma-Aldrich & F0503 \\
\hline Levamisole hydrochloride & Sigma-Aldrich & PHR1798 \\
\hline \multicolumn{3}{|l|}{ Experimental Models: Organisms/Strains } \\
\hline N2: wild type & Caenorhabditis Genetics Center & N2 \\
\hline HBR227: aptf-1(gk794) II. & [29] & HBR227 \\
\hline $\begin{array}{l}\text { HBR1361: goels304[pflp-11::SL1-GCaMP3.35-SL2::mKate2- } \\
\text { unc-54-3'UTR, unc-119(+)]. }\end{array}$ & This paper & HBR1361 \\
\hline $\begin{array}{l}\text { HBR1374: goels307[pflp-11::ArchT::SL2mKate2-unc-54- } \\
\text { 3'UTR,unc-119(+)]; goe/s304[pflp-11::SL1-GCaMP3.35- } \\
\text { SL2::mKate2-unc-54-3'UTR, unc-119(+)]. }\end{array}$ & This paper & HBR1374 \\
\hline $\begin{array}{l}\text { HBR1709: daf-16(mgDf50) l; goels304[pflp-11::SL1- } \\
\text { GCaMP3.35-SL2::mKate2-unc-54-3'UTR, unc-119(+)]. }\end{array}$ & $\begin{array}{l}\text { This paper and Caenorhabditis } \\
\text { Genetics Center }\end{array}$ & HBR1709 \\
\hline $\begin{array}{l}\text { HBR1753: wtfIs5[prab-3::NLS::GCaMP6s; prab- } \\
\text { 3::NLS::tagRFP] }\end{array}$ & $\begin{array}{l}\text { This paper, created from AML32 } \\
\text { (Andrew Leifer lab) [81] }\end{array}$ & HBR1753 \\
\hline $\begin{array}{l}\text { HBR1777: goels384 [pflp-11::egl-1::SL2-mkate2-flp- } \\
\text { 11-3'UTR, unc-119(+)]. }\end{array}$ & This paper & HBR1777 \\
\hline $\begin{array}{l}\text { HBR1807: goels232[psra-6::ReaChr::mKate2-unc-54- } \\
\text { 3'UTR, unc-119(+)]; goels304[pflp-11::SL1-GCaMP3.35- } \\
\text { SL2::mKate2-unc-54-3'UTR, unc-119(+)]. }\end{array}$ & This paper & HBR1807 \\
\hline $\begin{array}{l}\text { HBR1808: goels251[pmec-4::ReaChr::mKate2-unc-54- } \\
\text { 3'UTR, unc-119(+)]; goels304[pflp-11::SL1-GCaMP3.35- } \\
\text { SL2::mKate2-unc-54-3'UTR, unc-119(+)]. }\end{array}$ & This paper & HBR1808 \\
\hline $\begin{array}{l}\text { HBR1830: daf-2(e1370) III; goels304[pflp-11::SL1- } \\
\text { GCaMP3.35-SL2::mKate2-unc-54-3'UTR, unc-119(+)]. }\end{array}$ & $\begin{array}{l}\text { This paper and Caenorhabditis } \\
\text { Genetics Center }\end{array}$ & HBR1830 \\
\hline $\begin{array}{l}\text { HBR2005: aak-1(tm1944) III; aak-2(ok524) X; goels304[pflp- } \\
\text { 11::SL1-GCaMP3.35-SL2::mKate2-unc-54-3'UTR, unc-119(+)]. }\end{array}$ & $\begin{array}{l}\text { This paper and Caenorhabditis } \\
\text { Genetics Center }\end{array}$ & HBR2005 \\
\hline $\begin{array}{l}\text { HBR2006: daf-16(mgDf50) I; aak-1(tm1944) III; aak-2(ok524) X; } \\
\text { goels304[pflp-11::SL1-GCaMP3.35-SL2::mKate2-unc-54- } \\
\text { 3'UTR, unc-119(+)]. }\end{array}$ & $\begin{array}{l}\text { This paper and Caenorhabditis } \\
\text { Genetics Center }\end{array}$ & HBR2006 \\
\hline $\begin{array}{l}\text { HBR2007: daf-16(mgDf50) I; aak-1(tm1944) III; goels304[pflp- } \\
\text { 11::SL1-GCaMP3.35-SL2::mKate2-unc-54-3'UTR, unc-119(+)]. }\end{array}$ & $\begin{array}{l}\text { This paper and Caenorhabditis } \\
\text { Genetics Center }\end{array}$ & HBR2007 \\
\hline $\begin{array}{l}\text { HBR2009: daf-16(mgDf50) l; aak-2(ok524) X; goels304[pflp- } \\
\text { 11::SL1-GCaMP3.35-SL2::mKate2-unc-54-3'UTR, unc-119(+)]. }\end{array}$ & $\begin{array}{l}\text { This paper and Caenorhabditis } \\
\text { Genetics Center }\end{array}$ & HBR2009 \\
\hline $\begin{array}{l}\text { HBR2020: daf-16(mgDf50) I; aptf-1(gk794) II; aak-1(tm1944) III; } \\
\text { aak-2(ok524) X. }\end{array}$ & $\begin{array}{l}\text { This paper and Caenorhabditis } \\
\text { Genetics Center }\end{array}$ & HBR2020 \\
\hline HBR2030: aptf-1(gk794) II; aak-1(tm1944) III; aak-2(ok524) X. & $\begin{array}{l}\text { This paper and Caenorhabditis } \\
\text { Genetics Center }\end{array}$ & HBR2030 \\
\hline $\begin{array}{l}\text { AGD397: aak-1(tm1944) III; aak-2(ok524) X; uthEx202 } \\
\text { [crtc-1p::crtc-1 cDNA::tdTomato::unc-54 3'UTR + rol-6(su1006)] } \\
\text { (only non-rol-6 animals were used) }\end{array}$ & $\begin{array}{l}\text { Andrew Dillin lab, Caenorhabditis } \\
\text { Genetics Center [82] }\end{array}$ & AGD397 \\
\hline $\begin{array}{l}\text { CF1295: daf-16(mu86) I; daf-2(e1370) III; muEx108[pKL99-2(daf- } \\
\text { 16::GFP/daf16bKO) + pRF4(rol-6)] (only non-rol-6 animals } \\
\text { were used) }\end{array}$ & $\begin{array}{l}\text { Cynthia Kenyon lab, Caenorhabditis } \\
\text { Genetics Center [59] }\end{array}$ & CF1295 \\
\hline AM140: rm/s132 [punc-54::Q35::YFP] I. & $\begin{array}{l}\text { Richard Morimoto lab, Caenorhabditis } \\
\text { Genetics Center [67] }\end{array}$ & AM140 \\
\hline
\end{tabular}




\begin{tabular}{|c|c|c|}
\hline \multicolumn{3}{|l|}{ Continued } \\
\hline REAGENT or RESOURCE & SOURCE & IDENTIFIER \\
\hline $\begin{array}{l}\text { PD4251: ccls4251 [(pSAK2) pmyo-3::GFP::LacZ:: } \\
\text { NLS+(pSAK4)pmyo-3::mitochondrialGFP +dpy-20(+)] I. }\end{array}$ & $\begin{array}{l}\text { Andrew Fire lab, Caenorhabditis } \\
\text { Genetics Center [83] }\end{array}$ & PD4251 \\
\hline RW1596: myo-3(st386) V; stEx30[pmyo-3::GFP + rol-6(su1006)]. & $\begin{array}{l}\text { Robert Waterston lab, } \\
\text { Caenorhabditis Genetics Center [67] }\end{array}$ & RW1596 \\
\hline HBR1617: rm/s132[punc-54::Q35::YFP] l; aptf-1(gk794) II. & This paper and $[29,67]$ & HBR1617 \\
\hline $\begin{array}{l}\text { HBR1618: cc/s4251 [(pSAK2) pmyo-3::GFP::LacZ::NLS + (pSAK4) } \\
\text { pmyo-3::mitochondrial GFP + dpy-20(+)] l; aptf-1(gk794) Il; dpy- } \\
\text { 20(e1282) IV. }\end{array}$ & This paper and $[29,83]$ & HBR1618 \\
\hline $\begin{array}{l}\text { HBR1516: aptf-1(gk794) II; myo-3(st386) V; stEx30[pmyo-3::GFP + } \\
\text { rol-6(su1006)]. }\end{array}$ & This paper and $[29,67]$ & HBR1516 \\
\hline HBR2098: daf-18(ok480) IV. & $\begin{array}{l}\text { This paper and Caenorhabditis } \\
\text { Genetics Center }\end{array}$ & HBR2098 \\
\hline HBR2100: sir-2.1(ok434) IV. & $\begin{array}{l}\text { This paper and Caenorhabditis } \\
\text { Genetics Center }\end{array}$ & HBR210 \\
\hline HE1006: rol-6(su1006) II. & $\begin{array}{l}\text { Henry Epstein lab, Caenorhabditis } \\
\text { Genetics Center [84] }\end{array}$ & HE1006 \\
\hline $\begin{array}{l}\text { CF1724: daf-16(mu86) l; daf-2(e1370) III; muls105 [pdaf-16::GFP:: } \\
\text { daf-16 + rol-6(su1006)]. }\end{array}$ & $\begin{array}{l}\text { Cynthia Kenyon lab, Caenorhabditis } \\
\text { Genetics Center [59] }\end{array}$ & CF1724 \\
\hline $\begin{array}{l}\text { CF2093: daf-16(mu86) I; daf-2(e1370) III; muls131 [punc-119::GFP:: } \\
\text { daf-16 + rol-6(su1006)]. }\end{array}$ & $\begin{array}{l}\text { Cynthia Kenyon lab, Caenorhabditis } \\
\text { Genetics Center [85] }\end{array}$ & CF2093 \\
\hline $\begin{array}{l}\text { CF2093: daf-16(mu86) I; daf-2(e1370) III; muls131 [punc-119::GFP:: } \\
\text { daf-16 + rol-6(su1006)]. }\end{array}$ & $\begin{array}{l}\text { Cynthia Kenyon lab, Caenorhabditis } \\
\text { Genetics Center [85] }\end{array}$ & CF2093 \\
\hline $\begin{array}{l}\text { CF2102: daf-16(mu86) I; daf-2(e1370) III; muls126 [pmyo-3::GFP:: } \\
\text { daf-16 + rol-6(su1006)]. }\end{array}$ & $\begin{array}{l}\text { Cynthia Kenyon lab, Caenorhabditis } \\
\text { Genetics Center [85] }\end{array}$ & CF2102 \\
\hline $\begin{array}{l}\text { CF2005: daf-16(mu86) l; daf-2(e1370) III; muls120 [pges-1::GFP:: } \\
\text { daf-16 + rol-6(su1006)]. }\end{array}$ & $\begin{array}{l}\text { Cynthia Kenyon lab, Caenorhabditis } \\
\text { Genetics Center [85] }\end{array}$ & CF2005 \\
\hline $\begin{array}{l}\text { CF2570: daf-16(mu86) I; daf-2(e1370) III; muls142[pges-1::GFP:: } \\
\text { daf-16 + odr-1p::RFP]. }\end{array}$ & $\begin{array}{l}\text { Cynthia Kenyon lab, Caenorhabditis } \\
\text { Genetics Center [85] }\end{array}$ & CF2570 \\
\hline $\begin{array}{l}\text { HBR2158: daf-16(mgDf50) l; aak-2(ok524) X; tdEx618[ppgp-1:: } \\
\text { RFP+rol-6(su1006)]; goels304[pflp-11::SL1-GCaMP3.35-SL2:: } \\
\text { mKate2-unc-54-3'UTR, unc-119(+)]. }\end{array}$ & $\begin{array}{l}\text { This paper and Masamitsu } \\
\text { Fukuyama lab [56] }\end{array}$ & HBR2158 \\
\hline $\begin{array}{l}\text { HBR2159: daf-16(mgDf50) l; aak-2(ok524) X; tdEx589[ pMF342, } \\
\text { pmyo-3::GFP::aak-2::unc-54 3'UTR]+ppgp-1::RFP +rol-6(su1006)]. }\end{array}$ & $\begin{array}{l}\text { This paper and Masamitsu } \\
\text { Fukuyama lab [56] }\end{array}$ & HBR2159 \\
\hline $\begin{array}{l}\text { HBR2160: daf-16(mgDf50) I; aak-2(ok524) X; tdEx700[pMF380ppgp- } \\
\text { 1::GFP::aak-2::unc-54 3'UTR]+ppgp-1::RFP+rol-6(su1006)]. }\end{array}$ & $\begin{array}{l}\text { This paper and Masamitsu } \\
\text { Fukuyama lab [56] }\end{array}$ & HBR2160 \\
\hline $\begin{array}{l}\text { HBR2161: daf-16(mgDf50) I; aak-2(ok524) X; tdEx632[pMF308prgef- } \\
\text { 1::GFP:::aak-2:::unc-54 3'UTR+ppgp-1::RFP+rol-6(su1006)]. }\end{array}$ & $\begin{array}{l}\text { This paper and Masamitsu } \\
\text { Fukuyama lab [56] }\end{array}$ & HBR2161 \\
\hline $\begin{array}{l}\text { HBR2162: daf-16(mgDf50) I; aak-2(ok524) X; tdEx541[pMF312, } \\
\text { pdpy-7::GFP::aak-2::unc-54 3'UTR+ppgp-1::RFP+rol-6(su1006)]. }\end{array}$ & $\begin{array}{l}\text { This paper and Masamitsu } \\
\text { Fukuyama lab [56] }\end{array}$ & HBR2162 \\
\hline $\begin{array}{l}\text { HBR2163: daf-16(mgDf50) I; aak-2(ok524) X; tdEx679[pKS19, paak- } \\
\text { 2::aak-2::GFP::unc-86 3'UTR+ppgp-1::RFP+rol-6(su1006)]. }\end{array}$ & $\begin{array}{l}\text { This paper and Masamitsu } \\
\text { Fukuyama lab [56] }\end{array}$ & HBR2163 \\
\hline $\begin{array}{l}\text { HBR2173: daf-16(mgDf50), aak-2 (ok524) X; goeEx720 [pflp-11::aak- } \\
\text { 2a::SL2-mKate::flp-11 3'UTR, unc-119(+) + myo-2::mCherry]; } \\
\text { [ppgp-1::RFP+rol-6(su1006)]; goels304[pflp-111::SL1-GCaMP3.35- } \\
\text { SL2::mKate2-unc-54-3'UTR, unc-119(+)]. }\end{array}$ & $\begin{array}{l}\text { This paper and Caenorhabditis } \\
\text { Genetics Center }\end{array}$ & HBR2173 \\
\hline $\begin{array}{l}\text { HBR2151: daf-16(mgDf50), aak-2 (ok524) X; rrEx127[paak-2::aak- } \\
\text { 2+rol-6(su1006)]. }\end{array}$ & This paper and Richard Roy lab [63] & HBR2151 \\
\hline $\begin{array}{l}\text { HBR2152: daf-16(mgDf50), aak-2 (ok524) X; rrEx191[psulp-5::AAK- } \\
\text { 2+rol-6(su1006)] [63]. }\end{array}$ & This paper and Richard Roy lab [63] & HBR2152 \\
\hline $\begin{array}{l}\text { HBR2153: daf-16(mgDf50), aak-2 (ok524) X; rrEx123[pelt-2::aak- } \\
\text { 2+rol-6(su1006)] [63]. }\end{array}$ & This paper and Richard Roy lab [63] & HBR2153 \\
\hline $\begin{array}{l}\text { HBR2154: daf-16(mgDf50), aak-2 (ok524) X; rrEx120[psur-5::aak- } \\
\text { 2+rol-6(su1006)] [63]. }\end{array}$ & This paper and Richard Roy lab [63] & HBR2154 \\
\hline
\end{tabular}




\begin{tabular}{|c|c|c|}
\hline \multicolumn{3}{|l|}{ Continued } \\
\hline REAGENT or RESOURCE & SOURCE & IDENTIFIER \\
\hline $\begin{array}{l}\text { HBR2155: daf-16(mgDf50), aak-2 (ok524) X; rrEx122[punc-54::aak- } \\
\text { 2+rol-6(su1006)] [63]. }\end{array}$ & This paper and Richard Roy lab [63] & HBR2155 \\
\hline $\begin{array}{l}\text { HBR2156: daf-16(mgDf50), aak-2 (ok524) X; rrEx114[punc-119::aak- } \\
\text { 2+rol-6(su1006)] [63]. }\end{array}$ & This paper and Richard Roy lab [63] & HBR2156 \\
\hline $\begin{array}{l}\text { HBR2157: daf-16(mgDf50), aak-2 (ok524) X; rrEx193[pdpy-7p::aak- } \\
\text { 2+psur-5::GFP] [63]. }\end{array}$ & This paper and Richard Roy lab [63] & HBR2157 \\
\hline \multicolumn{3}{|l|}{ Oligonucleotides } \\
\hline For a list of primers see Table S7 & N/A & N/A \\
\hline \multicolumn{3}{|l|}{ Recombinant DNA } \\
\hline Plasmid K171: pmec-4::ReaChr::mKate2-unc-54-3'utr, unc-119(+) & This paper & K171 \\
\hline Plasmid K172: psra-6::ReaChr::mKate2-unc-54-3'utr, unc-119(+) & This paper & K172 \\
\hline Plasmid K214: pflp-11::ArchT::SL2-mKate2-unc-54-3'utr,unc-119(+) & This paper & K214 \\
\hline $\begin{array}{l}\text { Plasmid K216: pflp-11::SL1-GCaMP3.35-SL2::mKate2- } \\
\text { unc-54-3'utr, unc-119(+) }\end{array}$ & This paper & K216 \\
\hline Plasmid K281: pflp-11::egl-1::SL2-mKate2-flp-11-3'utr, unc-119(+) & This paper & K281 \\
\hline Plasmid K358: pflp-11::aak-2a::SL2-mKate2-flp-11 3'utr, unc-119(+) & This paper & K358 \\
\hline \multicolumn{3}{|l|}{ Software and Algorithms } \\
\hline MATLAB R2017a & Mathworks & $9.2 \cdot 0.538062$ \\
\hline OriginPro 2017 & Originlab & SR1 b9.4.1.354 (64-bit) \\
\hline Excel 2016 & Microsoft & MSO 16.0.4738.1000 (32-bit) \\
\hline Wormtracker & Urmersbach et al. [86] & Wormtracker \\
\hline ImageJ-win64 & Fiji - ImageJ & 1.52b, Java 1.6.0_24 (64-bit) \\
\hline Andor iQ2 & Andor & iQ 2.9.1 \\
\hline $\mathrm{R}$ & http://cran.r-project.org & R 3.3.2, GUI 1.68 \\
\hline NIS-Elements & Nikon & AR 5.02 .00 (64-bit) \\
\hline
\end{tabular}

\section{CONTACT FOR REAGENT AND RESOURCE SHARING}

Further information and requests for resources and reagents should be directed to and will be fulfilled by the Lead Contact, Henrik Bringmann (Henrik.Bringmann@mpibpc.mpg.de).

\section{EXPERIMENTAL MODEL AND SUBJECT DETAILS}

Worm maintenance and strains

C. elegans was grown on Nematode Growth Medium (NGM) agarose plates seeded with E. coli OP50 and were kept at 20 $0^{\circ} \mathrm{C}$ [87]. For crossings, the strains were genotyped using Duplex PCR genotyping of single worms [88]. Primer sequences used for Duplex PCR can be found in Table S7. Following C. elegans strains were used:

N2: wild-type

HBR227: aptf-1(gk794) II.

HBR1361: goels304[pflp-11::SL1-GCaMP3.35-SL2::mKate2-unc-54-3'UTR, unc-119(+)].

HBR1374: goels307[pflp-11::ArchT::SL2mKate2-unc-54-3'UTR,unc-119(+)]; goels304[pflp-11::SL1-GCaMP3.35-SL2:::mKate2unc-54-3'UTR, unc-119(+)].

HBR1709: daf-16(mgDf50) l; goe/s304[pflp-11::SL1-GCaMP3.35-SL2::mKate2-unc-54-3'UTR, unc-119(+)].

HBR1753: wtfIs5[prab-3::NLS::GCaMP6s; prab-3::NLS::tagRFP] (created from AML32 (a gift from Andrew Leifer) by backcrossing 2x into N2) [81].

HBR1777: goels384 [pflp-11::egl-1::SL2-mkate2-flp-11-3'UTR, unc-119(+)].

HBR1807: goels232[psra-6::ReaChr::mKate2-unc-54-3'UTR, unc-119(+)]; goels304[pflp-11::SL1-GCaMP3.35-SL2::mKate2unc-54-3'UTR, unc-119(+)].

HBR1808: goels251[pmec-4::ReaChr::mKate2-unc-54-3'UTR,unc-119(+)]; goels304[pflp-11::SL1-GCaMP3.35-SL2::mKate2unc-54-3'UTR, unc-119(+)]. 
HBR1830: daf-2(e1370) III; goels304[pflp-11::SL1-GCaMP3.35-SL2::mKate2-unc-54-3'UTR, unc-119(+)]. HBR2005: aak-1(tm1944) III; aak-2(ok524) X; goels304[pflp-11::SL1-GCaMP3.35-SL2::mKate2-unc-54-3'UTR, unc-119(+)]. HBR2006: daf-16(mgDf50) I; aak-1(tm1944) III; aak-2(ok524) X; goels304[pflp-11::SL1-GCaMP3.35-SL2::mKate2-unc-54-3'UTR, unc-119(+)].

HBR2007: daf-16(mgDf50) I; aak-1(tm1944) III; goels304[pflp-11::SL1-GCaMP3.35-SL2::mKate2-unc-54-3'UTR, unc-119(+)]. HBR2009: daf-16(mgDf50) I; aak-2(ok524) X; goels304[pflp-11::SL1-GCaMP3.35-SL2::mKate2-unc-54-3'UTR, unc-119(+)]. HBR2020: daf-16(mgDf50) I; aptf-1(gk794) II; aak-1(tm1944) III; aak-2(ok524) X. HBR2030: aptf-1(gk794) II; aak-1(tm1944) III; aak-2(ok524)X.

AGD397: aak-1(tm1944) III; aak-2(ok524) X; uthEx202 [crtc-1p::crtc-1 cDNA::tdTomato::unc-54 3'UTR + rol-6(su1006)] (only nonrol-6 animals were used) [82].

CF1295: daf-16(mu86) I; daf-2(e1370) III; muEx108[pKL99-2(daf-16::GFP/daf16bKO) + pRF4(rol-6)] (only non-rol-6 animals were used) [59].

AM140: rm/s132 [punc-54::Q35::YFP] I [67].

PD4251: ccls4251 [(pSAK2)pmyo-3::GFP::LacZ::NLS+(pSAK4)pmyo-3::mitochondrialGFP +dpy-20(+)] I [83].

RW1596: myo-3(st386) V; stEx30[pmyo-3::GFP + rol-6(su1006)] [69].

HBR1617: rmls132[punc-54::Q35::YFP] I; aptf-1(gk794) II.

HBR1618: ccls4251[(pSAK2) pmyo-3::GFP::LacZ::NLS + (pSAK4) pmyo-3::mitochondrial GFP + dpy-20(+)] l; aptf-1(gk794) II; dpy-20(e1282) IV.

HBR1516: aptf-1(gk794) II; myo-3(st386) V; stEx30[pmyo-3::GFP + rol-6(su1006)].

HBR2098: daf-18(ok480) IV created from RB712 by backcrossing $2 x$ into N2

HBR2100: sir-2.1(ok434) IV. created from VC199 by backcrossing 2x into N2

HE1006: rol-6(su1006) II [84].

CF1724: daf-16(mu86) I; daf-2(e1370) III; muls105 [pdaf-16::GFP::daf-16 + rol-6(su1006)] [59].

CF2093: daf-16(mu86) I; daf-2(e1370) III; muls131 [punc-119::GFP::daf-16 + rol-6(su1006)] [85]

CF2102: daf-16(mu86) I; daf-2(e1370) III; muls126 [pmyo-3::GFP::daf-16 + rol-6(su1006)] [85].

CF2005: daf-16(mu86) I; daf-2(e1370) III; muls120 [pges-1::GFP:::daf-16 + rol-6(su1006)] [85].

CF2570: daf-16(mu86) I; daf-2(e1370)III; muls142[pges-1::GFP::daf-16 + odr-1p::RFP] [85] .

HBR2158: daf-16(mgDf50) l; aak-2(ok524) X; tdEx618[ppgp-1::RFP+rol-6(su1006)]; goels304[pflp-11::SL1-GCaMP3.35SL2::mKate2-unc-54-3'UTR, unc-119(+)] [56].

HBR2159: daf-16(mgDf50) l; aak-2(ok524) X; tdEx589[ pMF342, pmyo-3::GFP::aak-2::unc-54 3'UTR]+ppgp-1::RFP+rol6(su1006)] [56].

HBR2160: daf-16(mgDf50) I; aak-2(ok524) X; tdEx700[pMF380ppgp-1::GFP::aak-2::unc-54 $\left.3^{\prime} U T R\right]+p p g p-1:: R F P+r o l-$ 6(su1006)] [56].

HBR2161: daf-16(mgDf50) I; aak-2(ok524) X; tdEx632[pMF308prgef-1::GFP::aak-2::unc-54 $3^{\prime} U T R+p p g p-1:: R F P+$ rol6(su1006)] [56].

HBR2162: daf-16(mgDf50) I; aak-2(ok524) X; tdEx541[pMF312, pdpy-7::GFP::aak-2::unc-54 $3^{\prime} U T R+p p g p-1:: R F P+r o l-$ 6(su1006)] [56].

HBR2163: daf-16(mgDf50) l; aak-2(ok524) X; tdEx679[pKS19, paak-2::aak-2::GFP::unc-86 $3^{\prime} U T R+p p g p-1:: R F P+$ rol6(su1006)] [56].

HBR2173: daf-16(mgDf50), aak-2 (ok524) X; goeEx720 [pflp-11::aak-2a::SL2-mKate::flp-11 $3^{\prime} U T R$, unc-119(+) + myo2::mCherry]; [ppgp-1::RFP+rol-6(su1006)]; goels304[pflp-11::SL1-GCaMP3.35-SL2::mKate2-unc-54-3'UTR, unc-119(+)].

HBR2151: daf-16(mgDf50), aak-2 (ok524) X; rrEx127[paak-2::aak-2+rol-6(su1006)] [63].

HBR2152: daf-16(mgDf50), aak-2 (ok524) X; rrEx191[psulp-5::AAK-2+rol-6(su1006)] [63].

HBR2153: daf-16(mgDf50), aak-2 (ok524) X; rrEx123[pelt-2::aak-2+rol-6(su1006)] [63].

HBR2154: daf-16(mgDf50), aak-2 (ok524) X; rrEx120[psur-5::aak-2+rol-6(su1006)] [63].

HBR2155: daf-16(mgDf50), aak-2 (ok524) X; rrEx122[punc-54::aak-2+rol-6(su1006)] [63].

HBR2156: daf-16(mgDf50), aak-2 (ok524) X; rrEx114[punc-119::aak-2+rol-6(su1006)] [63].

HBR2157: daf-16(mgDf50), aak-2 (ok524) X; rrEx193[pdpy-7p::aak-2+psur-5::GFP] [63].

\section{METHOD DETAILS}

Molecular biology and transgenic strain generation

We cloned all constructs into the pCG150 Vector that contains unc-119(+) [89] by using the Three Fragment Gateway System (Invitrogen, Carlsbad, CA). For verification, the cloned constructs were sequenced. The GCaMP3.35, the ReaChr, the ArchT and the egl-1 genes were expression-optimized for C. elegans [90]. The pflp-11::egl-1 transgene specifically expresses the apoptosis inducing protein EGL-1 in RIS. Thus, strains carrying this transgene have a genetic ablation of RIS. For AMPK rescue a cDNA corresponding to aak-2a was used and was obtained by synthesizing the gene based on the available gene sequence (Wormbase release WS266). We generated the transgenic strains by microparticle bombardment into unc-119(ed3) mutant worms and used phenotypic rescue of

e4 Current Biology 28, 3610-3624.e1-e8, November 19, 2018 
the unc phenotype as a selection marker [91, 92]. The insertions that were obtained were backcrossed two times against N2 to remove the unc-119(ed3) background. The following plasmids were created for this study:

K171: pmec-4::ReaChr::mKate2-unc-54-3'utr, unc-119(+)

K172: psra-6::ReaChr::mKate2-unc-54-3'utr, unc-119(+)

K214: pflp-11::ArchT::SL2-mKate2-unc-54-3'utr,unc-119(+)

K216: pflp-11::SL1-GCaMP3.35-SL2::mKate2-unc-54-3'utr, unc-119(+)

K281: pflp-11::egl-1::SL2-mKate2-flp-11-3'utr, unc-119(+)

K358: pflp-11::aak-2a::SL2-mKate2-flp-11 3'utr, unc-119(+)

Dauer pheromone extraction and dauer larvae generation

The crude dauer pheromone was extracted as described previously [93]. Briefly, worms were cultured in 1L of S-medium with resuspended OP50 at $25^{\circ} \mathrm{C}$. When the culture clarified as a consequence of bacteria depletion, additional OP50 was added. When cleared the second time, the supernatant was filtered and boiled to a solid crust. This crust was extracted three times with ethanol. After evaporation of the ethanol, the dauer pheromone was resuspended in $1 \mathrm{~mL}$ of sterile water and stored at $-20^{\circ} \mathrm{C}$. A $10 \mu \mathrm{L}$ drop of water containing dauer pheromone was placed on the solidified agarose, which contained the microchambers. The drop was placed after sealing the chambers with a coverslip. The drop was pipetted onto the surface that did not contain any microchambers and subsequently diffused into the agar. To obtain dauer larvae, NGM plates were left to starve at $25^{\circ} \mathrm{C}$ and daily controlled for occurrence of dauer larvae. 3 days after the first dauers occurred, the starved NGM plate was chunked to a fresh unseeded NGM plate and the dauers were allowed to crawl off that chunk. From there, individual dauer larvae were isolated and used for behavioral experiments

Long-term imaging

For long-term imaging, agarose microchamber imaging was used [34,35]. Briefly, box-shaped indentations in agarose hydrogel were cast using a PDMS mold. The chambers were then filled with worms and for fed conditions with bacteria, and sealed with a glass coverslip. 3\% agarose dissolved in S-Basal [94] was used as a hydrogel in all experiments to starve worms and bacteria except for the experiments were we investigated behavior in the presence of growth medium. To obtain growth medium conditions we mixed Nematode Growth Medium [94] and S-Basal 1:1, added 3\% agarose, and casted microchambers from this mix. To obtain dead bacteria, the OP50 lawn was pasteurized on seeded NGM plates for $3 \mathrm{hr}$ in an oven set to $70^{\circ} \mathrm{C}$. For pheromone experiments, $10 \mu \mathrm{L}$ of dauer pheromone extract was added on top of the agarose microchamber, which then diffused into the agarose. Behavioral and functional calcium imaging [29, 86] was performed simultaneously. In short, we used an Andor iXon (512x512 pixels) EMCCD camera and LED illumination (CoolLed) using standard GFP filter sets. The exposure time was set to $5 \mathrm{~ms}$. The EMCCD "TTL fire out" signal was used to trigger the LED illumination in order to illuminate only during exposure. The $490 \mathrm{~nm}$ light intensity was $2.00 \mathrm{~mW} / \mathrm{mm}^{2}$ using a $20 \mathrm{x}$ objective and $0.60 \mathrm{~mW} / \mathrm{mm}^{2}$ using a $10 \mathrm{x}$ objective. EM gain was set to 100 . Using these parameters, we obtained image sequences with clearly identifiable worm outlines and measurable neuronal calcium transients.

Typically, 8-12 individual fields in close vicinity were filmed. Adult worms were cultured in $700 \mu \mathrm{m} \times 700 \mu \mathrm{m} \times 45 \mu \mathrm{m}(\mathrm{X}$ length $\mathrm{x}$

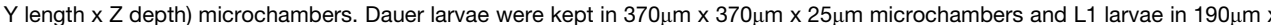
$190 \mu \mathrm{m} \times 15 \mu \mathrm{m}$ unless otherwise mentioned. For adults and dauer larvae, one imaged field included an individual worm in a single microchamber. For L1s, one filmed field can include up to four worms in adjacent microchambers. Adults were imaged using the 10x objective. L1s and dauer were imaged with the 20x objective. An automatic stage (Prior Proscan 2 or 3 ) moved repeatedly to the microchambers using low acceleration speeds. The frame rate we obtained was 0.1 frame/s unless otherwise noted. Worms were filmed either continuously or in series of movies for optogenetic experiments.

In the L1 experiments, pretzel stage eggs were picked with an eyelash or eyebrow hair to an empty NGM plate to remove remaining bacteria. From this empty NGM plate, the eggs were transferred to the microchamber again using a clean hair. Image acquisition was started $24 \mathrm{hr}$ after hatching. Dauers were obtained from plates that were left to starve at $25^{\circ} \mathrm{C}$. To isolate individual dauers, we cut out agarose chunks of the starved plates and transferred them to an empty NGM plate and allowed the worms to crawl off the agarose chunk. Individual dauers were pipetted with $1-5 \mu \mathrm{L}$ of water to the microchamber. With a pick, the dauers were distributed over the microchambers so that each dauer was placed into its own chamber. We assayed dauers that were 3 days old. Adults for starvation, daf-2(e1370) mutants and its controls were treated with 5-fluoro-2-deoxyuridine (FUdR, Sigma-Aldrich) before the experiments. For this, L4s were transferred to seeded NGM plates containing 50uM FUdR and 50mg/L kanamycin (Sigma-Aldrich) [64]. $24 \mathrm{hr}$ after the FUdR treatment, we transferred the adults to the microchamber. The worms were either transferred with a worm pick into a microchamber filled with OP50 for feeding experiments or they were pipetted in a 1-5 $\mu \mathrm{L}$ drop of water to a microchamber for starvation experiments. For starvation experiments, worms were kept without food for $24 \mathrm{hr}$ in the empty microchambers before image acquisition. For daf-2(e1370) mutant experiments, imaging was started $35 \mathrm{hr}$ after shifting the temperature from $15^{\circ} \mathrm{C}$ to $23^{\circ} \mathrm{C}$. Imaging was started $6 \mathrm{hr}$ after the pheromone application. Imaging was started $3 \mathrm{hr}$ after the microchamber was prepared. To allow identification of the non-pumping period, additional DIC images were collected. 
Optogenetics and light stimulation

All optogenetic experiments using ReaChr and ArchT were performed inside microchambers [29]. Two days before the experiments, the strains were grown on NGM plates supplemented with $0.2 \mathrm{mM}$ all-trans retinal (ATR, Sigma-Aldrich). The agarose that was used for microchamber fabrication was also supplemented with ATR of the same concentration. A dual GFP/mCherry excitation/dichroic plus GFP emission filter set (Chroma) was used to allow simultaneous GCaMP imaging and optogenetic manipulation. To activate ReaChr and ArchT, the LED light intensity at $585 \mathrm{~nm}$ was set to $0.47 \mathrm{~mW} / \mathrm{mm}^{2}$ using a $20 x$-objective and $0.14 \mathrm{~mW} / \mathrm{mm}^{2}$ using a 10x-objective. The light intensities were measured using a light voltmeter (PM100A, Thorlabs).

To test for responsiveness to stimulation, we applied blue light irradiation at $490 \mathrm{~nm}$ with an intensity of $3 \mathrm{~mW} / \mathrm{mm}^{2}$ to arrested L1 larvae inside the microfluidic compartments. To evoke a behavioral response we used blue light, which triggers an endogenous lightavoidance response. For these experiments, L1 worms were starved for $24 \mathrm{hr}$ inside microchambers $(110 \mu \mathrm{m} \times 110 \mu \mathrm{m} \times 10 \mu \mathrm{m})$ and imaged with 2 frames/s. After $1 \mathrm{~min}$ of baseline imaging, the animals were illuminated for $3 \mathrm{~min}$ and followed by an additional $1 \mathrm{~min}$ imaging without illumination. Each animal was assayed up to 16 times with at least $1 \mathrm{hr}$ between two blue light irradiations to allow the worm to recover from the stimulus. To extract sleep-wake-differences of responsiveness, the worm's behavioral state was classified post hoc into either "wake" or "sleep." The worm was classified as "wake," when it was never in a sleep bout during the imaging sequence $3.5 \mathrm{~min}$ before the stimulation (see below) throughout the baseline measurement and was classified as "sleep" when it was continuously in a sleep bout during the $3.5 \mathrm{~min}$ before stimulation. Only data from time points that continuously met the criteria for being asleep or awake during the $3.5 \mathrm{~min}$ before stimulation were taken for the sleep and wake analysis, the other data were excluded. These excluded data were those time points during which the worm showed episodes of both sleep and wake during the 3.5 min of stimulation. The specific numbers of experiments and data exclusions are specified as follows: For Figure 3A, a total of 600 measurements were made. 446 (74.3\%) were counted as "wake," 98 (16.3\%) were counted as "sleep" and 56 (9.3\%) could not be assigned to "wake" or "sleep" and were excluded. For Figure 3C with ATR, a total of 125 measurements were made. 70 (56.0\%) were counted as "wake," 19 (15.2\%) were counted as "sleep" and $36(28.8 \%)$ could not be assigned to "wake" or "sleep" and were excluded. For Figure $3 \mathrm{C}$ without ATR, a total of 273 measurements were made. $110(40.3 \%)$ were scored as "wake," $40(14.7 \%)$ were scored as "sleep" and 123 (40.1\%) could not be assigned to "wake" or "sleep" and were excluded. For Figure 3D with ATR, a total of 167 measurements were made. 101 (60.5\%) were counted as "wake," 42 (25.1\%) were counted as "sleep" and $24(14.4 \%)$ could not be assigned to "wake" or "sleep" and were excluded. For Figure 3D without ATR, a total of 297 measurements were made. $69(23.2 \%)$ were counted as "wake," 85 (28.6\%) were counted as "sleep" and $143(48.1 \%)$ could not be assigned to "wake" or "sleep" and were excluded. Overall for Figures 3C and 3D, a total of 862 measurements were made. $350(40.6 \%)$ were counted as "wake," $186(21.6 \%)$ were counted as "sleep" and $326(37.8 \%)$ could not be assigned to "wake" or "sleep" and were excluded. For Figure 3E with stimulus, a total of 180 measurements were made. $80(44.4 \%)$ were counted as "wake," $38(21.1 \%)$ were counted as "sleep" and 62 (34.4\%) could not be assigned to "wake" or "sleep" and were excluded. For Figure 3E without stimulus, a total of 131 measurements were made. 32 (24.4\%) were counted as "wake," 71 (54.2\%) were counted as "sleep" and $28(21.4 \%)$ could not be assigned to "wake" or "sleep" and were excluded.

To measure responsiveness to stimulation, the slope of the linear fit of the movement speed over the first minute of stimulation was used to measure the velocity increase. These velocity increase measurements were averaged for every worm and were subsequently averaged over all worms measured. Data was statistically compared using the Wilcoxon signed rank test for paired samples so as to compare sleep and wake responses.

To test for homeostasis, RIS was optogenetically inhibited in arrested L1 larvae. flp-11::ArchT expressing larvae were starved for $24 \mathrm{hr}$ in the microchambers and imaged for $3 \mathrm{hr}$. During the first hour, baseline data was recorded without illumination. For another hour the data was recorded under green light illumination. The third hour was recorded without green light. For statistical comparison (two-sample t test) between the condition with supplemented ATR and without ATR, we used averaged data ranging from $2.5-7.5 \mathrm{~min}$ after the end of green light stimulation.

To test for reversibility, arrested L1 larvae that express either sra-6::ReaChr or mec-4::ReaChr were used $24 \mathrm{hr}$ after hatching in the absence of food. Each animal was imaged for $30 \mathrm{~min}$ and illuminated after $15 \mathrm{~min}$ for $1500 \mathrm{~ms}$ with green light. This was repeated up to 6 times with a delay of at least $1 \mathrm{hr}$ to allow the worm to recover from the stimulus. The reversibility recordings were classified post hoc in "awake" or "asleep." During each data point, the worm was classified as awake, when it spent less than $25 \%$ in a sleep bout during the $15 \mathrm{~min}$ of baseline measurement and was classified as asleep when it spent at least $95 \%$ of the time in a sleep bout during the $3.5 \mathrm{~min}$ time interval before the optogenetic activation started. Data that could not get clearly classified with the above criteria were excluded from the analysis. Awake and asleep data was averaged for every worm to give one value for wake and one for sleep. Data was then averaged across all worms. For statistical comparisons (two-sample $t$ test), we averaged data from the $5 \mathrm{~min}$ after the end of optogenetic stimulation.

Image Analysis

We extracted two parameters from the acquired images: 1) worm locomotion velocity: Two methods were used: A) The worm's head position was determined either manually or automatically with a home-made MATLAB routine that detects the position of RIS in the head of the animal, or the centroid of the entire body, similar to the one previously described [86] and calculated with a conversion factor to obtain the velocity in $\mu \mathrm{m} / \mathrm{s}$. This method was applied for Figures 2, 3B, 4C-4F, 5G, S3E, and S6C. B) Sleep bouts were extracted using frame subtraction using a MATLAB routine similarly as previously described [95]. This method was applied for Figure S4. 2) RIS depolarization as measured by GCaMP3 intensity: Using the coordinates for RIS position as determined by the MATLAB

e6 Current Biology 28, 3610-3624.e1-e8, November 19, 2018 
routine, RIS GCaMP3 intensity was extracted from the images. The RIS GCaMP3 intensity was calculated as the mean of the 30 highest pixel intensities in an 11 pixel $x 11$ pixel square around the RIS cell body. Pan-neuronal nuclear GCaMP6s intensity was calculated as the mean of the 500 highest pixel intensities in a 69pixel $\times 69$ pixel region of interest around the center of the head neurons. The intensities were subsequently normalized to obtain $\Delta F / F$.

We analyzed sleep bouts in movies with $3 \mathrm{hr}$ recording time. To score for sleep, the velocity data was smoothed using a $1^{\text {st }}$ degree polynomial local regression model over 25 time points using the in-build smooth function in MATLAB. To be scored as a sleep bout, it had to last at least $3 \mathrm{~min}$ with a smoothed velocity below $0.5 \mu \mathrm{m} / \mathrm{s}$ for adults and dauers and below $3 \mu \mathrm{m} / \mathrm{s}$ for arrested L1 larvae. In L1 larvae in the presence of food, a sleep bout was defined as a smoothed locomotion velocity below $1.2 \mu \mathrm{m} / \mathrm{s}$ for at least $2 \mathrm{~min}$. To be scored as sleep using frame subtraction, intensity counts had to be below $20 \%$ of the average intensity. These cutoff parameters were determined empirically [95]. For sleep amount comparisons the time spent in a sleep bout was divided by the total time. For developing L1 larvae in the presence of food, lethargus was defined by the non-pumping period. As a representative L1 wake phase we took $3 \mathrm{hr}$ before lethargus onset.

For averaging RIS activity and velocity, data was aligned to the sleep bout onset. RIS activity and velocity starting $15 \mathrm{~min}$ before bout onset until 30min after sleep bout onset were selected for display. To obtain a flat baseline, selections that have sleeping bouts within the $15 \mathrm{~min}$ before the bout onset were excluded from the alignment. The averaged RIS activities $5 \mathrm{~min}$ before and $5 \mathrm{~min}$ after the bout onset were compared using the Wilcoxon signed rank test for paired samples. The displayed RIS $\Delta F / F$ data of individual animals was smoothed with a 5-point-running-average.

Lifespan and survival assays

To isolate L1 larvae for lifespan and survival assays, eggs were synchronized using bleaching of a mixed population of worms and collected in standard M9 buffer [96]. The worms were allowed to hatch and to arrest overnight. The animals were then kept in the dark, at $20^{\circ} \mathrm{C}$ on a slowly spinning rotator in $2 \mathrm{~mL}$ Eppendorf tubes. At defined time intervals, we took samples and transferred the worms to fresh seeded NGM plates. We scored after 5-20 min the percentage of living worms to measure survival. A worm was scored as alive, when it was able to move on the plate. To analyze recovery, we kept the worms on the plates for $2-4$ days at $20^{\circ} \mathrm{C}$ and then scored the percentage of worms that had re-entered development and had grown to at least larval stage L3 after feeding. At least 50 worms were scored at each time point. For comparisons, the time points " $50 \%$ alive" and " $50 \%$ recovering" were defined. These values represent the days when the survival/recovering curves reach $50 \%$ surviving or recovering worms [65].

For survival measurements of arrested L1s in the presence of food, FUdR was used to cause the arrest. FUdR was added to a final concentration of $50 \mu \mathrm{M}$ to the synchronized worm culture in $\mathrm{M} 9$ at $20^{\circ} \mathrm{C}$ at day 4 . At day 5 , at least 100 worms were pipetted to NGM plates seeded with $E$. coli and containing $50 \mu \mathrm{M}$ of FUdR, with a worm density of roughly 20 worms per plate. We kept the plates at $20^{\circ} \mathrm{C}$ in dark and scored every two or three days for viability. Worms were identified as dead if they didn't shown locomotion after several gentle mechanical stimulations by pick. Dead worms were removed from the plates. Living worms were gently transferred to fresh FUdR NGM plates if any fungi contamination appeared on the plates. Worms that had dried out after crawling off the agar surface were excluded from the analysis.

For adult lifespan assays on food, a standard lifespan protocol was used [64]. In brief, a synchronized worm culture was grown until young adulthood. At least 50 worms were transferred to freshly seeded NGM plates with a worm density of roughly 10 worms per plate. Worms were kept at $20^{\circ} \mathrm{C}$ in dark and scored every two or three days for viability. They were classified as alive if they showed locomotion or, if quiescent, responded to gentle mechanical stimulation exerted by a platinum wire pick. Living worms were transferred to fresh NGM plates every two days in the first two weeks to separate them from their offspring until they had stopped laying eggs. Worms that had dried out after crawling off the agar surface or that died after leaking out through the vulva were excluded from the analysis. The lifespan assays of starving adults were performed similar to the lifespan assay on food. The differences were that

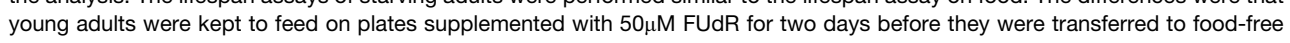
plates containing again $50 \mu \mathrm{M}$ FUdR.

To correlate sleep amount with survival, we kept arrested larvae inside agarose microchambers without food at $20^{\circ} \mathrm{C}$ in dark. The microchambers with $\mathrm{L} 1$ larvae were prepared as described above. When the agar appeared dry after a few days, we re-moisturized the microchamber with $20 \mu \mathrm{L}$ of S-Basal containing $100 \mathrm{u} / \mathrm{mL}$ Nystatin (Sigma Aldrich). Nystatin was added to prevent fungal growth. Worms were imaged after two days of arrest using DIC time-lapse microscopy with a frame rate of 0.1 frame/s to quantify sleep. Using a home-made MATLAB script, the worm's centroid was determined and its velocity measured. The resulting plot was smoothed over 40 time points. To be scored sleeping, the worm's smoothened velocity had to be below $40 \%$ of the average velocity for at least $2 \mathrm{~min}$. The time spent sleeping was divided by the total imaging time, averagely for each worm from more than $35 \mathrm{hr}$ of time lapse footage recorded between day 2 and day 4 after starvation. A linear fit was calculated from worms within the range: mean (lifespan) $\pm \mathrm{SD}_{\text {(ifespan). }}$ The others were excluded as outliers.

Quantification of bacteria consumption

To quantify bacterial consumption in FUdR-arrested larvae, FUdR was added to a final concentration of $50 \mu \mathrm{M}$ to the 3 day synchronized worm culture for $24 \mathrm{hr}$. Worms were transferred in agarose microchambers filled with OP50 that had been transformed with a GFP plasmid (pFPV25.1) [34, 35, 80]. Food consumption was measured by quantifying GFP fluorescence over time. As a control we used bacteria-filled chambers that did not contain worms. With standard GFP filter sets, 20x objective, $490 \mathrm{~nm}$ light (with $2.00 \mathrm{~mW} / \mathrm{mm}^{2}$ intensity), $5 \mathrm{~ms}$ exposure time and $50 \mathrm{EM}$ gain we quantified GFP signals one and three days after microchamber 
preparation. For statistical comparisons (two-sample t test), we averaged the pixel intensities of the chambers and calculated the change in fluorescent intensity between day one and three.

To compare bacterial consumption of FUdR-arrested and reproductive growing L1s, worms were cultured in agarose microchambers as described above. Food consumption was measured by quantifying GFP fluorescence with 10x objective over 200 frames ( $1 \mathrm{frame} / \mathrm{min}$ ) at the 4-day old arrested L1. At this time, worms had approximately similar body sizes. To determine the body size of L1s, we assumed an elongated cylinder shape of the worm. The volume of each worm was then calculated from the body length and the radius of the worm [97]. For statistical comparison (two-sample t test), we normalized the fluorescence intensity change during the first 200 frames of each chamber to the corresponding mean worm volume.

Quantification of aging markers

Mitochondrial fission, poly-Q35 aggregation, and muscle morphology was quantified with fluorescence microscopy similar to what was previously described [65]. $L 1$ arrested larvae $(n=10-15)$ were imaged every two to three days with a spinning disc fluorescence microscope equipped with a 488nm laser (Andor Revolution on Nikon TiE). Imaging was performed after immobilization with $25 \mathrm{mM}$ Levamisole and mounting of the worms between a sandwich of a coverslip and a $1 \mathrm{~mm}$-thick 4\% agarose in M9 pad [94]. Images were acquired and scored using Andor IQ Software. Imaging conditions for the Andor iXon camera were an EM gain of 100 and exposure time of $15-25 \mathrm{~ms}$. Laser power was adjusted for each condition. Using $100 x$ objective or $60 x$ objective, $z$ stacks were recorded to image all focal planes of each worm. To score morphology of muscle fibers and mitochondria, body muscle cells in two of DL, DR, VL, VR bundles (in the head or body for fibers or mitochondria, respectively) were analyzed by scrolling through all stacks. First, 50 striated myofilaments in head were scored manually if they were defective (i.e., showed the morphological disruption or disorganization) [98]. Mitochondria were classified manually into three fission levels: tubular, intermediate fragmentation, or complete fragmentation as in a previous study [65]. The number of poly-Q35 aggregates was also scored manually for each worm by scrolling through all $\mathrm{z}$ stacks. Similar to how aggregates were scored in previous studies, we defined an aggregate as a discrete structure that is isolated from the surrounding fluorescence with a discernible boundary and that falls within a typical size between 1 to $5 \mu \mathrm{m}$ [65, 67]. All aging marker experiments were replicated twice. Two independent observers, which were not blinded to the genotype, and who agreed on the final numbers, scored each experiment.

\section{QUANTIFICATION AND STATISTICAL ANALYSIS}

Quantification is described in the Method Details. Statistical tests used were Student's t tests (two-sided), Mann-Whitney U test, Wilcoxon rank tests for paired samples, log-rank tests and Fisher's exact test using Origin software or MATLAB. For multiple comparisons in Figures 4 (multiple genotypes) and S4 (multiple tissue-specific rescues) significance was confirmed by using the Benjamini-Hochberg Procedure with a false discovery rate of $5 \%$. The specific tests used are described in the figure captions and the Results. The graphs show mean \pm SEM unless noted otherwise. The boxplots show individual data points, the box represents the $25 \%-75 \%$ range, the whiskers the $10 \%-90 \%$ range, the thin gray line is the median and the bold black line is the mean. For each experiment at least two biological replicates were performed. Detailed results for lifespan and survival assays can be found in Tables S1-S5. A detailed list of the numbers of replicates for each experiment can be found in Table S6. 
Current Biology, Volume 28

Supplemental Information

Sleep Counteracts Aging Phenotypes

to Survive Starvation-Induced

Developmental Arrest in C. elegans

Yin Wu, Florentin Masurat, Jasmin Preis, and Henrik Bringmann 
Figure S1

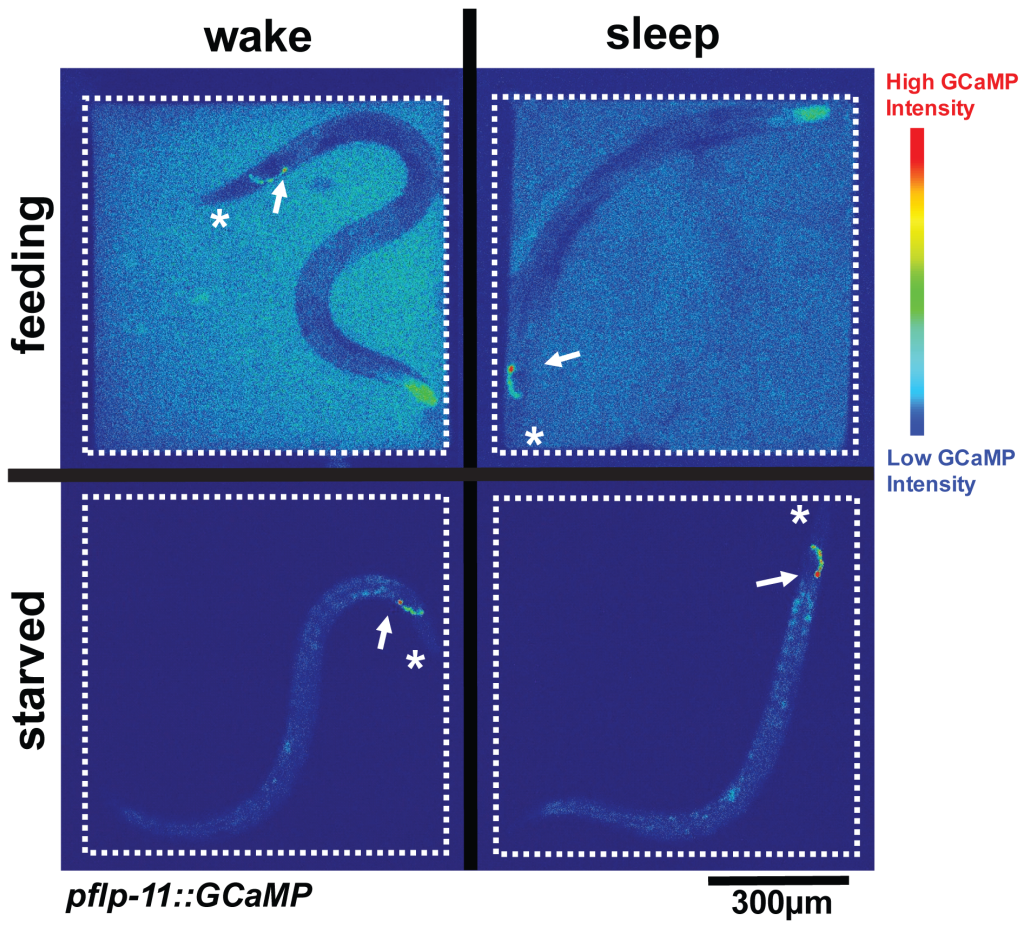

Figure S1. Calcium and behavioral imaging in agarose microchambers. Related to Figure 1 and Figure 2. Shown are fluorescence images of adult worms expressing GCaMP3 in the sleepactive neuron RIS. The pictures are false-colored to visualize calcium activity. The border of the agarose microchamber is marked by a white dotted line. The asterisk indicates the worm's nose, the arrow the sleep neuron RIS. The left panels show wake worms, the right panels sleeping worms. Note the relaxed body posture and increased RIS GCaMP intensity during sleep. The top panels show worms feed on OP50. The bacteria are visible due to their auto-fluorescence. On the bottom panels, the worms are starved. 
Figure S2
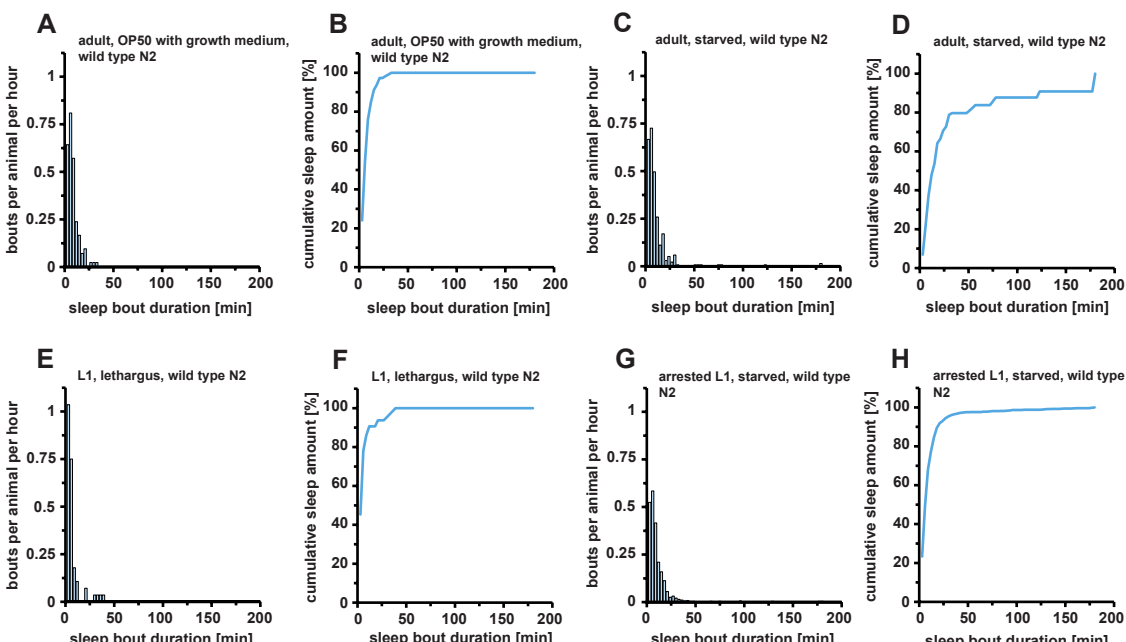

G

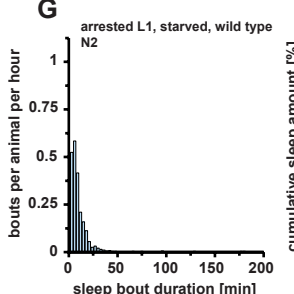

$\mathrm{H}$
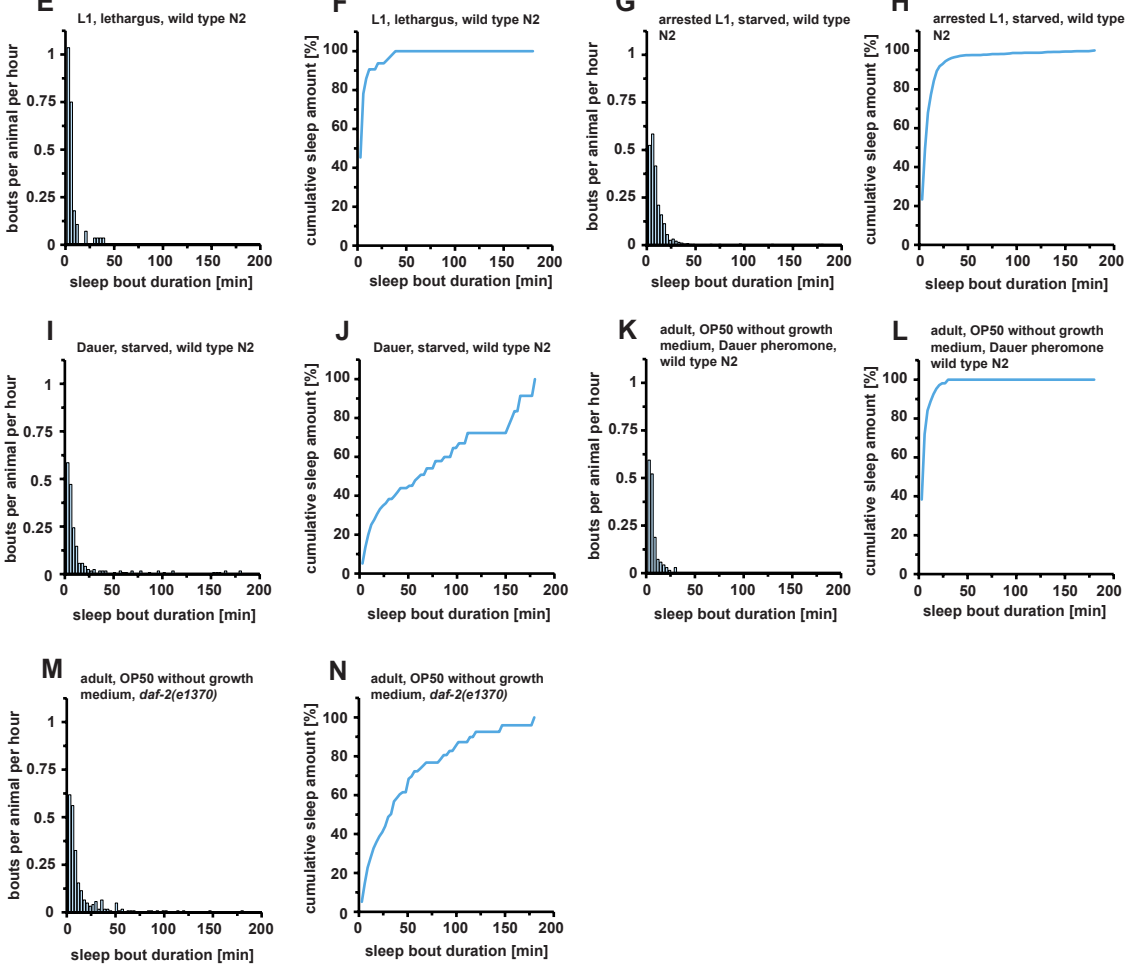

Figure S2. Distribution of sleep bout lengths and their contribution to total sleep amount. Related to Figure 1 and Figure 2. Shown are number of bouts per animal per hour as well as cumulative sleep amount as a function of bout length to show which bout lengths contribute how much to total sleep amount. Note that most sleep occurs in shorter bouts except for dauer larvae and $d a f-2(-)$, which show also longer sleep bouts that contribute substantially to total sleep amount. (A-B) Adults with growth medium, (C-D) starved adults, (E-F) L1 lethargus, (G-H) starved L1 arrested, (I-J) dauer without food, (K-L) adults feeding on starved bacteria and dauer pheromone, (M-N) daf-2(-) adults feeding on starved bacteria. 
Figure S3
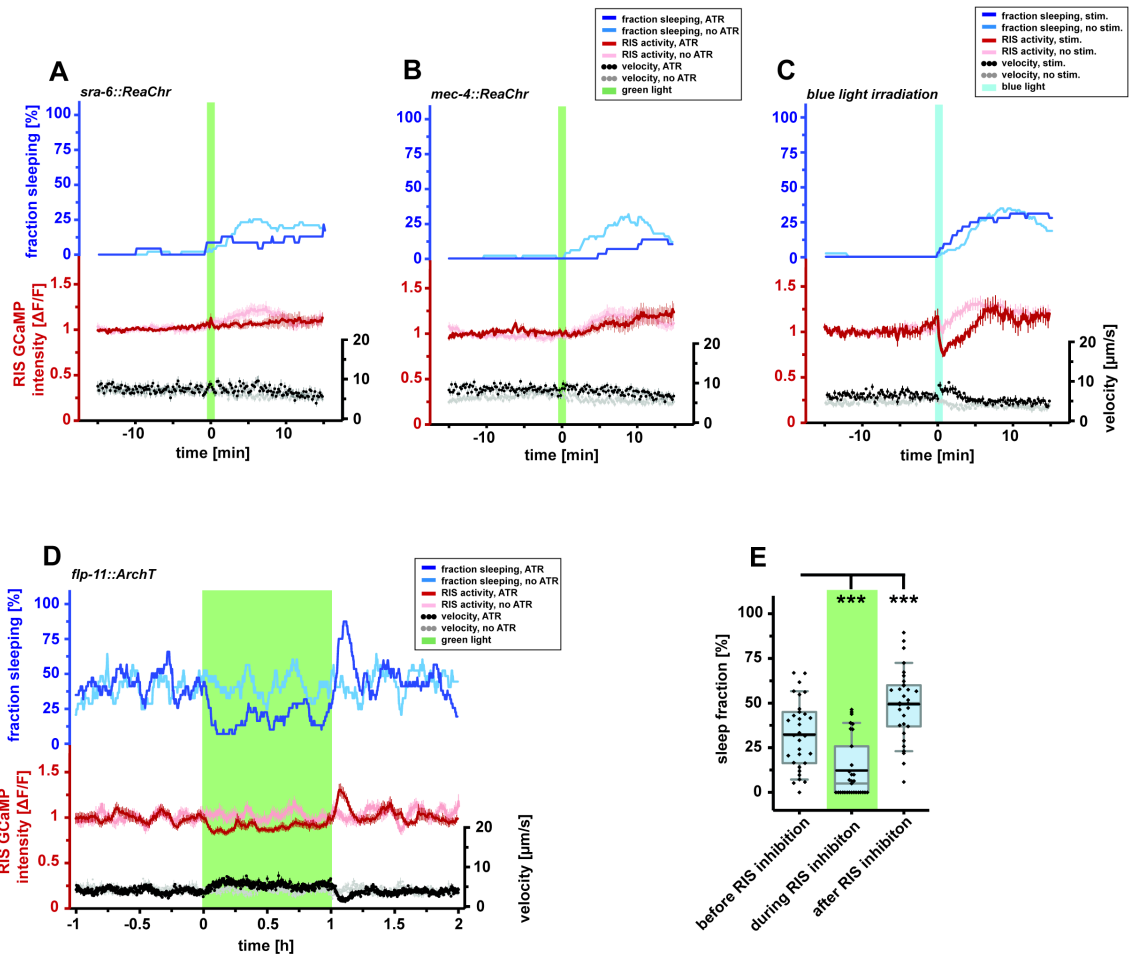

Figure S3. Control experiments for characterizing larval sleep during starvation-induced arrest. Related to Figure 3. (A-B) ASH and mechano-sensory neurons activation during wake in arrested L1 larvae. During wake, there is no detectable action from ASH nociceptive neurons (A) or mec-4 expression mechano-sensory neurons (B). RIS activity is shown in red (control without ATR in light red), speed in black (control without ATR in gray), and the fraction of sleeping animals in blue (control without ATR in light blue). (A-C) ASH and mechano-sensory neurons activation during wake in arrested L1 larvae. During wake, there is no detectable action from ASH nociceptive neurons (A), mec-4 expression mechano-sensory neurons (B) or from noxious blue light irradiation (C). RIS activity is shown in red (control without ATR or without stimulation in light red), speed in black (control without ATR or without stimulation in gray), the fraction of sleeping animals in blue (control without ATR or without stimulation in light blue), green light illumination in green and noxious blue light irradiation in teal. (D-E) flp-11::ArchT activation efficiently reduces RIS activity and consequently inhibits sleep. (D) Complete documentation of homeostasis experiment. RIS activity is shown in red (control without ATR in light red), speed in black (control without ATR in gray), and the fraction of sleeping animals in blue (control without ATR in light blue). (E) Statistical comparison of the different $1 \mathrm{~h}$ long periods. During green light illumination the sleep fraction was reduced by $62.0 \pm 0.5 \%, * * * p<0.001$, and after illumination the sleep fraction increased by $52.5 \pm 0.1 \%, * * * p<0.001$, compared to the sleep fraction before the illumination. Comparisons were made using the paired Wilcoxon rank test. $\mathrm{n}=32$ worms. 
Figure S4

A

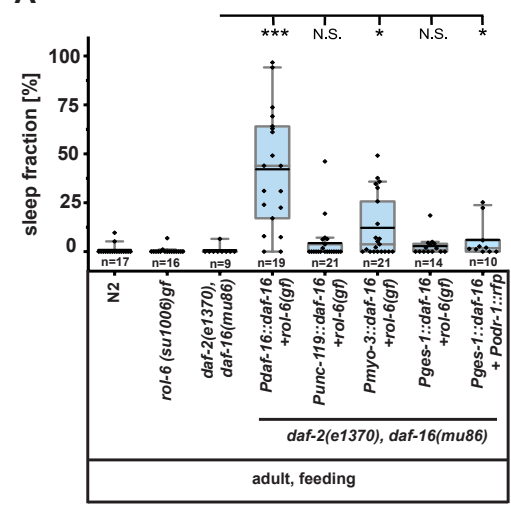

B

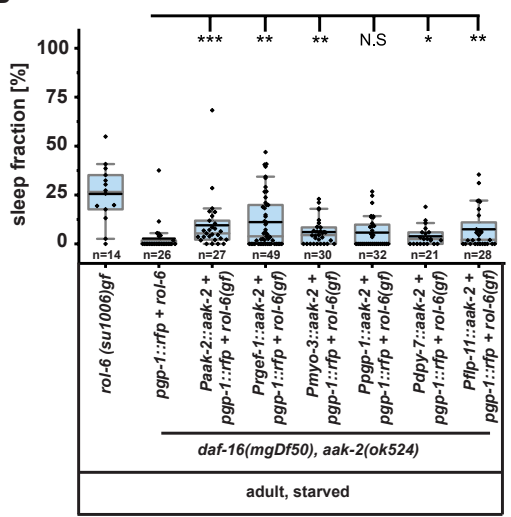

C

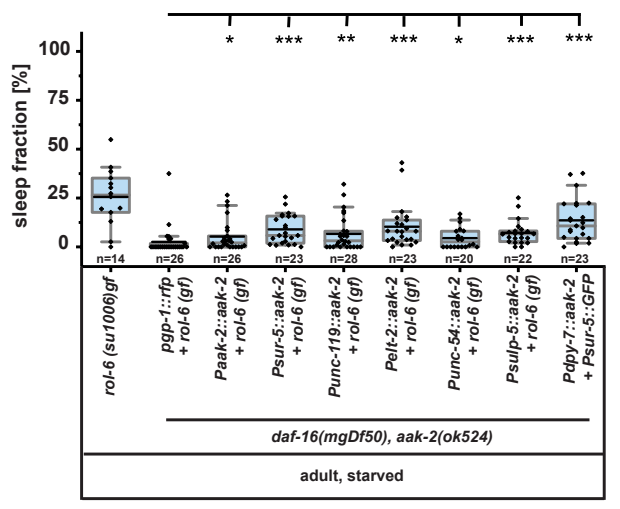

Figure S4. Rescue experiments for FoxO and AMPK. Related to Figure 4. (A) Rescue experiments using inserted transgenes of daf-16 shows weak rescue only in muscle and inconsistent recue in the intestine. daf-16 was expressed from its endogenous promoter, in neurons (Punc-119), in body wall muscle (Pmyo-3) and in the intestine (Pges-1). Most transgenes carry a rol-6(su1006) transformation marker, the roller phenotype does not affect sleep, Median sleep fraction was $0 \%$ for wild type $\mathrm{N} 2$ and rol-6(su1006), the same data is displayed in panel B. Median sleep fraction for rescue experiments was $0 \%$ for the background genotype daf-2(e1370)/daf$16(\mathrm{mu} 86), 44 \%$ for rescue using the endogenous promoter $(* * * \mathrm{p}<0.001), 0 \%$ for pan-neuronal rescue, $4 \%$ for muscle rescue $(* p<0.05), 1 \%$ and $2 \%$ for intestinal rescue dependent on the 
transgenic array $(\mathrm{p}=0.11$ and $* \mathrm{p}<0.05$, respectively). daf-2(e1370)/daf-16(mu86) data was taken from DIC movies collected for the same animals that were analyzed for Figure $4 \mathrm{C}$ and is the same as used in Figure S4B. (B) Rescue experiments using extrachromosomal transgenes of aak-2 shows weak rescue across tissues, which was expressed from its endogenous promotor, in neurons (Prgef-1), in body wall muscle (Pmyo-3), in the intestine (Ppgp-1), in the hypodermis (Pdpy-7), and in RIS (Pflp-11). Transgenes carry a rol-6(su1006) and intestinal red fluorescence ( $p g p-1: \because r f p)$ transformation marker. For comparison, a wild type background is shown, which only expresses a transformation marker. It is the same comparison as shown in Figure S4D. Median sleep fraction was $0 \%$ for daf-2(mgDf50)/aak-2(ok524) carrying the transformation markers only, $5 \%$ for rescue by endogenous promoter $(* * * p<0.001), 4 \%$ for pan-neuronal rescue $(* * p<0.01), 4 \%$ for muscle rescue $(* * p<0.01), 0 \%$ for intestine, $2 \%$ for hypodermis $(* p<0.05)$, and $2 \%$ for RIS $(* * p<0.01)$. (C) Additional rescue experiments using extrachromosomal transgenes of aak-2 with different transformation markers and promoters shows weak rescue across tissues, which was expressed from its endogenous promotor, ubiquitously (Psur-5), in neurons (Punc-119), in the intestine (Pelt$2)$, in muscle (Punc-54), in the hypodermis (Pdpy-7), and in the excretory cell (Psulp-5). Transgenes carry a rol-6(su1006) or other transformation marker. For comparison, a wild type background is shown, which only expresses a transformation marker. It is the same comparison as shown in Figure S4C. Median sleep fraction was $0 \%$ for daf-2(mgDf50)/aak-2(ok524) carrying the transformation markers only, $2 \%$ for rescue by the endogenous promoter $(* p<0.001), 6 \%$ for ubiquitous expression (***p $<0.001), 3 \%$ for pan-neuronal rescue $(* * p<0.01), 8 \%$ for intestine $(* * * p<0.001), 3 \%$ for muscle rescue $(* p<0.05), 7 \%$ for the excretory cell $(* * * p<0.001)$ and $11 \%$ for hypodermis $(* * * \mathrm{p}<0.001)$. The low rescue effects obtained after using the endogenous aak-2 and sur-5 promoters are consistent with previous measurements using these transgenes. Perhaps the weak rescue can be explained by weak transmission of the array[S1]. The number of worms assayed (n) is displayed underneath each box plot. For statistical comparisons, MannWhitney $\mathrm{U}$ test was used to calculate the $\mathrm{p}$ value and significance was confirmed by BenjaminiHochberg Procedure for multiple comparisons. 
Figure S5
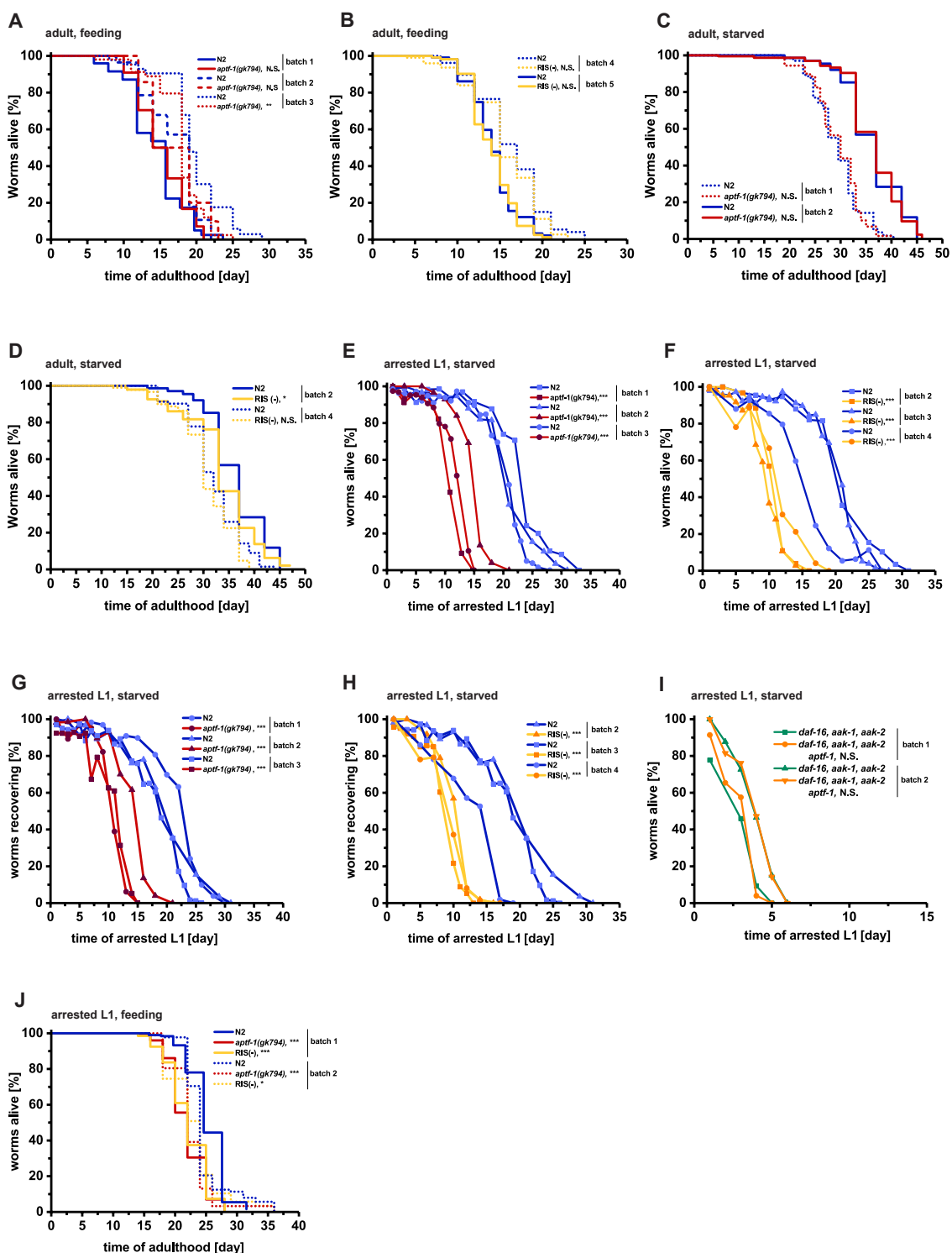

Figure S5. Sleep is required to survive larval starvation. Related to Figure 5. Supplementary figure containing additional replicates related to Figure 5. The biological replicates are indicated as different batches with different lines or symbols. (A) Adult lifespan of feeding wild type (blue) and aptf-1 mutants (red). No consistent significant difference could be shown. (B) Lifespan of feeding wild type (blue) and RIS(-) (yellow) adult worms were not significantly different for the two replicates. (C) No significant difference was detected for the starved adult lifespan of wild type and aptf-1(gk794), (D) and also not between starved wild type and RIS(-) adult worms. (EF) L1-arrested starved aptf-1 (gk794) mutants and RIS ablated worms show substantially reduced survival in all replicates latest from day 16. (G-H) All replicates of arrested L1 aptf-1 (gk794) and RIS-ablated worms showed a significant decline in the ability to re-enter development compared to N2 worms when fed. Data are significant latest from day 12. (I) Starved arrested L1 aak1(tm 1944)/aak-2(ok524)/daf-16(mgDf50)/aptf-1(gk794) quadruple mutants showed no reduced lifespan compared to aak-1(tm1944)/aak-2(ok524)/daf-16(mgDf50) triple mutants. (J) aptf1 (gk794) and RIS(-) L1s arrested on FUdR have a decreased survival compared to wild type worms in the presence of food. The corresponding data, significance values and tests for all replicates are shown in Tables S1-S5. 


\section{Figure S6}
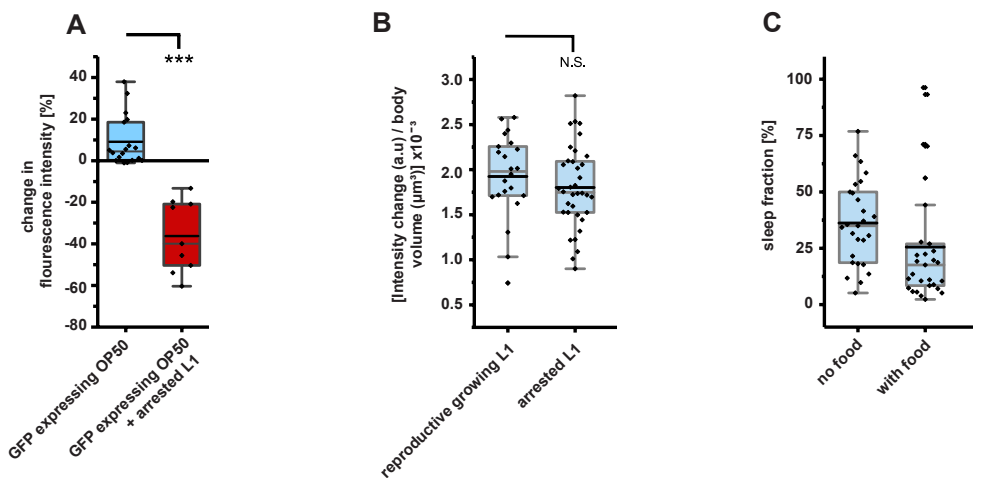

Figure S6. Arrested L1 larvae consume bacteria. Related to Figure 5. (A) The fluorescence intensity change of GFP expressing OP50 was measured in agarose microchambers. The two-day reduction in fluorescence is significantly different comparing microchambers containing arrest L1s to microchambers without worms (with worms: $36.3 \pm 5.8 \%$ decrease, $\mathrm{n}=9$ microchambers, without worms: $9.1 \pm 2.8 \%$ increase, $\mathrm{n}=18$ microchambers, ${ }^{* * *} \mathrm{p}<0.001$, Mann-Whitney U test). (B) Arrested L1 larvae consume similar amounts of bacteria as reproductive growing L1 worms. The change in fluorescence intensity of OP50 GFP bacteria within 200 min was normalized to worm volume, to compensate for variability in size, which is caused by increased growth in nonarrested worms. The time window was chosen because size differences were still modest. No significant difference in bacterial consumption was detected for reproductive growing L1s (with a mean of $1.92 \times 10^{-3}$ arbitrary units $\left./ \mu \mathrm{m}^{3}, \mathrm{n}=22\right)$ and FUdR-arrested L1s (mean $=1.8 \times 10^{-3}$ arbitrary units $/ \mu \mathrm{m} 3, \mathrm{n}=36$ ), $\mathrm{p}=0.33$, two-sample t-test. (C) Sleep fraction of starved and feeding FUdR arrested L1s in wildtype. Starved L1s with FUdR (4 to 5 days-old) had a median sleep fraction of $34.9 \%, \mathrm{n}=27$. Median sleep fraction of the feeding L1s with FUdR (4 to 5 days-old) is $17.5 \%, \mathrm{n}$ $=29$. 
Table S1

\begin{tabular}{|c|c|c|c|c|c|}
\hline \multicolumn{6}{|c|}{ Lifespan of adult, feeding } \\
\hline Strain & $\begin{array}{l}\text { Experiment } \\
\text { batch } \\
\text { (in Figure) }\end{array}$ & \# scored/ \# total & $\begin{array}{c}\text { Mean of lifespan } \\
\text { (days) }\end{array}$ & $\begin{array}{l}\text { Compared } \\
\text { to }\end{array}$ & $\begin{array}{c}p \text { value against } \\
\text { control } \\
\text { (log-rank test) }\end{array}$ \\
\hline wildtype N2 & $\begin{array}{c}1 \text { (Figure } \\
5 \mathrm{~A})\end{array}$ & $44 / 50$ & 14.64 & & \\
\hline aptf-1(gk794) & $\begin{array}{c}1 \text { (Figure } \\
5 \mathrm{~A})\end{array}$ & $43 / 50$ & 15.29 & N2 Batch1 & 0.71, N.S. \\
\hline wildtype N2 & 2 & $28 / 50$ & 16.92 & & \\
\hline aptf-1(gk794) & 2 & $12 / 50$ & 16.91 & N2 Batch2 & 0.70, N.S. \\
\hline wildtype N2 & 3 & $39 / 50$ & 19.74 & & \\
\hline aptf-1(gk794) & 3 & $42 / 50$ & 17.82 & N2 Batch3 & $0.003, * *$ \\
\hline wildtype N2 & 4 & $72 / 100$ & 16.30 & & \\
\hline RIS(-) & 4 & $75 / 102$ & 15.54 & N2 Batch4 & 0.23, N.S. \\
\hline wildtype N2 & $\begin{array}{c}5 \text { (Figure } \\
5 \mathrm{~B})\end{array}$ & $94 / 120$ & 14.22 & & \\
\hline RIS(-) & $\begin{array}{l}5 \text { (Figure } \\
5 \mathrm{~B})\end{array}$ & $86 / 120$ & 14.07 & N2 Batch5 & 0.71, N.S. \\
\hline \multicolumn{6}{|c|}{ Lifespan of adult, starved } \\
\hline wildtype N2 & $\begin{array}{c}1 \text { (Figure } \\
5 \mathrm{C})\end{array}$ & $60 / 100$ & 29.81 & - & \\
\hline aptf-1(gk794) & $\begin{array}{l}1 \text { (Figure } \\
5 \mathrm{C})\end{array}$ & $63 / 100$ & 29.46 & N2 Batch1 & 0.65, N.S. \\
\hline wildtype N2 & 2 & $52 / 200$ & 36.21 & & \\
\hline aptf-1(gk794) & 2 & $94 / 200$ & 36.33 & N2 Batch2 & 0.96 , N.S. \\
\hline RIS(-) & 2 & $83 / 200$ & 33.62 & N2 Batch2 & $0.04, *$ \\
\hline wildtype N2 & $\begin{array}{l}3 \text { (Figure } \\
5 \mathrm{D})\end{array}$ & $85 / 100$ & 31.96 & & \\
\hline RIS(-) & $\begin{array}{c}3 \text { (Figure } \\
5 \mathrm{D})\end{array}$ & $84 / 95$ & 30.64 & N2 Batch3 & 0.06, N.S. \\
\hline
\end{tabular}

Table S1. Overview of all replicates of the adult lifespan. Related to Figure 5. Displayed are the strains used, the experimental / replicates, the scored animals from the total number (the missing worms were censored), the mean lifespan estimated from the log-rank test, against which wild type data was tested and the $p$ value of the log-rank test. 
Table S2

\begin{tabular}{|c|c|c|c|c|c|c|}
\hline \multicolumn{7}{|c|}{ Survival of arrested L1, starved } \\
\hline Strain & $\begin{array}{l}\text { Experiment } \\
\text { batch } \\
\text { (in Figure) }\end{array}$ & $\begin{array}{c}\min . \\
\text { scored/counting }\end{array}$ & $\begin{array}{l}50 \% \\
\text { alive at } \\
\text { (days) }\end{array}$ & $\begin{array}{l}\text { Compared } \\
\text { to }\end{array}$ & $\begin{array}{c}\Delta \\
50 \% \\
\text { alive }\end{array}$ & $\begin{array}{c}p \text { value against } \\
\text { control } \\
\text { (Fisher's exact test) }\end{array}$ \\
\hline wildtype N2 & 1 & $>50$ & 22.89 & & & \\
\hline aptf-1(gk794) & 1 & $>50$ & 10.62 & N2 Batch1 & $-53.6 \%$ & $\begin{array}{l}<0.001, * * * \\
\text { from day } 11\end{array}$ \\
\hline wildtype N2 & 2 & $>50$ & 20.06 & & & \\
\hline aptf-1 $(g k 794)$ & 2 & $>50$ & 14,69 & N2 Batch2 & $-26.8 \%$ & $\begin{array}{l}<0.001, * * * \\
\text { from day } 16\end{array}$ \\
\hline RIS(-) & 2 & $>50$ & 10.3 & N2 Batch2 & $-48.7 \%$ & $\begin{array}{l}<0.001, * * * \\
\text { from day } 10\end{array}$ \\
\hline wildtype N2 & 3 (Figure 5E) & $>50$ & 20.70 & & & \\
\hline aptf-1(gk794) & 3 (Figure 5E) & $>50$ & 12.07 & N2 Batch3 & $-41.7 \%$ & $\begin{array}{l}<0.001, * * * \\
\text { from day } 11\end{array}$ \\
\hline RIS(-) & 3 (Figure 5E) & $>50$ & 9.41 & N2 Batch3 & $-54.5 \%$ & $\begin{array}{l}<0.001, * * * \\
\text { from day } 9\end{array}$ \\
\hline wildtype N2 & 4 & $>50$ & 14.85 & & & \\
\hline RIS(-) & 4 & $>50$ & 9.93 & N2 Batch4 & $-33.1 \%$ & $\begin{array}{l}<0.001, * * * \\
\text { from day } 12\end{array}$ \\
\hline
\end{tabular}

Table S2. Overview of all replicates of the starved arrested L1 lifespans. Related to Figure 5. Displayed are the strains used, the batch / replicate, the scored worms, median survival, against which wild type data was tested, the reduction in lifespan compared to wildtype and the $p$ value and from which day on there was a significant difference in the Fisher's exact test.

Table S3

\begin{tabular}{|c|c|c|c|c|c|c|}
\hline \multicolumn{7}{|c|}{ Ability to re-enter development of arrested L1 when fed, starved } \\
\hline Strain & $\begin{array}{l}\text { Experiment } \\
\text { batch } \\
\text { (in Figure) }\end{array}$ & $\begin{array}{c}\text { min. } \\
\text { scored/counting }\end{array}$ & $\begin{array}{c}50 \% \\
\text { recover } \\
\text { at (days) }\end{array}$ & Compared to & $\begin{array}{c}\Delta \\
50 \% \\
\text { alive }\end{array}$ & $\begin{array}{c}p \text { value against } \\
\text { control } \\
\text { (Fisher's exact test) }\end{array}$ \\
\hline wildtype N2 & 1 & $>50$ & 22.68 & & & \\
\hline aptf-1(gk794) & 1 & $>50$ & 10.49 & N2 Batch1 & $-53.7 \%$ & $\begin{array}{l}<0.001, * * * \\
\text { from day } 11\end{array}$ \\
\hline wildtype N2 & 2 & $>50$ & 19.42 & & & \\
\hline aptf-1 (gk794) & 2 & $>50$ & 14.48 & N2 Batch2 & $-25.4 \%$ & $\begin{array}{l}<0.001, * * * \\
\text { from day } 16\end{array}$ \\
\hline RIS(-) & 2 & $>50$ & 10.28 & N2 Batch2 & $-47.1 \%$ & $\begin{array}{l}<0.001, * * * \\
\text { from day } 10\end{array}$ \\
\hline wildtype N2 & 3 (Figure 5F) & $>50$ & 18.74 & & & \\
\hline aptf-1 (gk794) & 3 (Figure 5F) & $>50$ & 11.37 & N2 Batch3 & $-39.3 \%$ & $\begin{array}{l}<0.001, * * * \\
\text { from day } 12\end{array}$ \\
\hline RIS(-) & 3 (Figure 5F) & $>50$ & 8.53 & N2 Batch3 & $-54.5 \%$ & $\begin{array}{c}<0.001, * * * \\
\text { from day } 8\end{array}$ \\
\hline wildtype N2 & 4 & $>50$ & 14.12 & & & \\
\hline RIS(-) & 4 & $>50$ & 9.09 & N2 Batch4 & $-35.6 \%$ & $\begin{array}{l}<0.001, * * * \\
\text { from day } 12\end{array}$ \\
\hline
\end{tabular}

Table S3. Overview of all replicates of the capacity to recover from the $L 1$ arrest. Related to Figure 5. Displayed are the strains used, the experimental batch / replicate, the scored animals, median recovery rate, against which wild type data set was tested, the reduction in lifespan compared to wildtype and the $p$ value and from which day on there was a significant difference in the Fisher's exact test. 
Table S4

\begin{tabular}{|c|c|c|c|c|c|}
\hline \multicolumn{6}{|c|}{ Survival of arrested L1 of quadruple mutants, starved } \\
\hline Strain & $\begin{array}{c}\text { Experiment } \\
\text { batch } \\
\text { (in Figure) }\end{array}$ & $\begin{array}{c}\min . \\
\text { scored/counting }\end{array}$ & $\begin{array}{l}50 \% \\
\text { alive at } \\
\text { (days) }\end{array}$ & $\begin{array}{l}\text { Compared } \\
\text { to }\end{array}$ & $\begin{array}{c}p \text { value against } \\
\text { control } \\
\text { (Fisher's exact test) }\end{array}$ \\
\hline $\begin{array}{l}\text { aak-1(tm1944), aak- } \\
\text { 2(ok524), daf-16(mgDf50) }\end{array}$ & 1 & $>60$ & 2.74 & & \\
\hline $\begin{array}{l}\text { aak-1(tm1944), aak- } \\
\text { 2(ok524), daf-16(mgDf50), } \\
\text { aptf-1(gk794) }\end{array}$ & 1 & $>60$ & 3.14 & $\begin{array}{l}\text { Triple } \\
\text { mutant } \\
\text { Batch1 }\end{array}$ & $\begin{array}{l}>0.05 \\
\text { N.S. }\end{array}$ \\
\hline $\begin{array}{l}\text { aak-1(tm1944), aak- } \\
\text { 2(ok524), daf-16(mgDf50) }\end{array}$ & $\begin{array}{l}2 \text { (Figure } \\
5 \mathrm{H} \text { ) }\end{array}$ & $>60$ & 3.85 & & \\
\hline $\begin{array}{l}\text { aak-1(tm1944), aak- } \\
\text { 2(ok524), daf-16(mgDf50), } \\
\text { aptf-1(gk794) }\end{array}$ & $\begin{array}{l}2 \text { (Figure } \\
5 \mathrm{H})\end{array}$ & $>60$ & 3.9 & $\begin{array}{l}\text { Triple } \\
\text { mutant } \\
\text { Batch2 }\end{array}$ & $\begin{array}{l}>0.05 \\
\text { N.S. }\end{array}$ \\
\hline
\end{tabular}

Table S4. Overview of all replicates of the lifespan of IIS and AMPK mutants. Related to Figure 5. Overview of all replicates of the lifespan of IIS and AMPK mutants. Displayed are the strains used, the experimental batch / replicate, the scored animals, median recovery rate, against which wild type data set was tested, the reduction in lifespan compared to wildtype and the $p$ values and significance in the Fisher's exact test.

Table S5

\begin{tabular}{|l|c|c|c|l|c|c|}
\hline \multicolumn{7}{|c|}{ Survival of FUdR-arrested L1, feeding } \\
\hline \multicolumn{1}{|c|}{ Strain } & $\begin{array}{c}\text { Experiment } \\
\text { batch } \\
\text { (in Figure) }\end{array}$ & scored/total & $\begin{array}{c}\text { Mean of } \\
\text { lifespan } \\
\text { (days) }\end{array}$ & Compared to & $\begin{array}{c}\Delta \\
50 \% \\
\text { alive }\end{array}$ & $\begin{array}{c}p \text { value against } \\
\text { control } \\
\text { (log-rank test) }\end{array}$ \\
\hline wildtype N2 & $\begin{array}{c}\text { 1 (Figure } \\
\text { 5I) }\end{array}$ & $143 / 200$ & 25.70 & & & \\
\hline aptf-1(gk794) & $\begin{array}{c}1 \text { (Figure } \\
5 \mathrm{I})\end{array}$ & $140 / 200$ & 21.86 & N2 Batch1 & $-14.5 \%$ & $<0.001, * * *$ \\
\hline RIS(-) & $\begin{array}{c}1 \text { (Figure } \\
5 \mathrm{I})\end{array}$ & $135 / 200$ & 22.05 & N2 Batch1 & $-14.2 \%$ & $<0.001, * * *$ \\
\hline wildtype N2 & 2 & $88 / 101$ & 24.63 & & & \\
\hline aptf-1(gk794) & 2 & $92 / 101$ & 22.5 & N2 Batch2 & $-8.6 \%$ & $<0.001, * * *$ \\
\hline RIS(-) & 2 & $100 / 144$ & 23.04 & N2 Batch2 & $-6.5 \%$ & $0.017, *$ \\
\hline
\end{tabular}

Table S5. Overview of all replicates of the lifespan of FUdR-arrested L1s. Related to Figure 5. Displayed are the strains used, the experimental batch / replicate, the scored animals, the mean survival, against which wild type data set was tested, the reduction in lifespan compared to wildtype and the $p$ values and the significance in the log-rank test. 
Table S6

\begin{tabular}{|c|c|c|}
\hline Experiment & No. of biological replicates & Comments \\
\hline Figure 1J & 4 & \\
\hline Figure 1K & 7 & \\
\hline Figure 1L & 3 & \\
\hline Figure $1 \mathrm{M}$ & 7 & \\
\hline Figure $1 \mathrm{~N}$ & 5 & \\
\hline Figure 10 & 3 & \\
\hline Figure $2 \mathrm{~A}$, wt with growth medium & 4 & \\
\hline Figure $2 \mathrm{~A}, \operatorname{aptf}-1(-)$ with growth medium & 4 & \\
\hline Figure 2A, wt without growth medium & 2 & \\
\hline Figure 2A, aptf-1(-) without growth medium & 2 & \\
\hline Figure 2A, wt with growth medium, dead OP50 & 3 & \\
\hline Figure $2 \mathrm{~A}$, aptf-1(-) with growth medium, dead OP50 & 2 & \\
\hline Figure 2B, wt, adult feeding & 2 & \\
\hline Figure 2B, aptf-1(-), adult feeding & 2 & \\
\hline Figure 2B, Pflp-1l:EGL-1, adult feeding & 6 & \\
\hline Figure 2B, wt, adult starved & 9 & \\
\hline Figure 2B, aptf-1(-), adult starved & 3 & \\
\hline Figure 2B, Pflp-11:EGL-1, adult starved & 6 & \\
\hline Figure 2C, wt, non-starved L1, before lethargus & 3 & \\
\hline Figure 2C, aptf-1(-), non-starved L1, before lethargus & 3 & \\
\hline Figure 2C, wt, non-starved L1, during lethargus & 3 & \\
\hline Figure 2C, aptf-1(-), non-starved L1, during lethargus & 3 & \\
\hline Figure 2D, wt, arrested L1 & 8 & \\
\hline Figure 2D, aptf-1(-), arrested L1 & 4 & \\
\hline Figure 2D, Pflp-11:EGL-1, arrested L1 & 2 & \\
\hline Figure 2E, wt, Dauer & 8 & \\
\hline Figure 2E, aptf-I(-), Dauer & 4 & \\
\hline Figure 2E, Pflp-11:EGL-I, Dauer & 4 & \\
\hline Figure 2F, wt, Dauer Pheromone & 5 & \\
\hline Figure 2F, aptf-1(-), Dauer Pheromone & 3 & \\
\hline Figure 3A, B: 2 & 2 & \\
\hline Figure $3 \mathrm{C}$ with ATR & 2 & \\
\hline Figure $3 \mathrm{C}$ without $\mathrm{ATR}$ & 2 & \\
\hline Figure 3D with ATR & 2 & \\
\hline Figure 3D without ATR & 2 & \\
\hline Figure 3E with ATR & 4 & \\
\hline Figure 3E without ATR & 3 & \\
\hline Figure $3 \mathrm{~F}$ & 2 & \\
\hline Figure 4B & 6 & \\
\hline Figure 4C wt & 2 & \\
\hline Figure 4C daf-2(-) & 9 & \\
\hline Figure 4C daf-2(-); aptf-1(-) & 4 & \\
\hline Figure 4C daf-2(-); daf-16(-) & 6 & \\
\hline Figure 4D wt & 9 & \\
\hline Figure 4D daf-16(-) & 4 & \\
\hline Figure 4D aak-1(-); aak-2(-) & 7 & \\
\hline Figure 4D daf-16(-); aak-1(-) & 4 & \\
\hline Figure 4D daf-16(-); aak-2(-) & 2 & \\
\hline Figure 4D daf-16(-); aak-1(-);aak-2(-) & 3 & \\
\hline Figure 4E wt & 8 & \\
\hline Figure 4E daf-16(-) & 2 & \\
\hline Figure 4E aak-1(-); aak-2(-) & 2 & \\
\hline Figure 4E daf-16(-); aak-1(-) & 2 & \\
\hline Figure 4E daf-16(-); aak-2(-) & 4 & \\
\hline Figure 4E daf-16(-); aak-1(-); aak-2(-) & 6 & \\
\hline Figure 4F wt & 2 & \\
\hline Figure $4 \mathrm{~F}$ daf-18(-) & 2 & \\
\hline Figure $4 \mathrm{~F}$ sir $2.1(-)$ & 2 & \\
\hline Figure $5 \mathrm{~A}$ adult $\mathrm{wt}$, feeding + Figure $\mathrm{S} 5 \mathrm{~A}$ & 3 & \\
\hline Figure $5 \mathrm{~A}$ adult aptf- $-1(-)$, feeding + Figure S5A & 3 & \\
\hline Figure 5B adult wt, feeding + Figure S5B & 2 & \\
\hline Figure 5B adult RIS(-), feeding + Figure S5B & 2 & \\
\hline Figure 5C adult wt, starved + Figure S5C & 2 & \\
\hline Figure $5 \mathrm{C}$ adult aptf $-1(-)$, starved + Figure $\mathrm{S} 5 \mathrm{C}$ & 2 & \\
\hline Figure 5D adult wt, starved + Figure S5D & 2 & \\
\hline Figure 5D adult RIS(-), starved + Figure S5D & 2 & \\
\hline Figure 5E L1 wt, starved + Figure S5E-F & 3 & \\
\hline
\end{tabular}




\begin{tabular}{|c|c|c|}
\hline Figure 5E L1 aptf-1(-), starved + Figure S5E-F & 3 & \\
\hline Figure 5E L1 RIS(-), starved + Figure S5E-F & 3 & \\
\hline Figure 5F L1 wt, starved + Figure S5G-H & 3 & \\
\hline Figure 5F L1 aptf-1(-), starved + Figure S5G-H & 3 & \\
\hline Figure 5F L1 RIS(-), starved + Figure S5G-H & 3 & \\
\hline Figure 5G wt, starved & 5 & \\
\hline Figure 5H daf-16(-); aak-1(-); aak-2(-), starved + Figure S5I & 2 & \\
\hline Figure $5 \mathrm{H}$ daf-16(-), aak-1(-), aak-2(-), aptf-1(-), starved + Figure S5I & 2 & \\
\hline Figure 5I L1 wt, FUDR, feeding + Figure S5J & 2 & \\
\hline Figure 5I L1 aptf-1(-), FUDR, feeding + Figure S5J & 2 & \\
\hline Figure 5I L1 RIS(-), FUDR, feeding + Figure S5J & 2 & \\
\hline Figure 6A wt & 2 & \\
\hline Figure 6A aptf-1(-) & 2 & \\
\hline Figure $6 \mathrm{C} \mathrm{wt}$ & 2 & \\
\hline Figure 6C aptf-1(-) & 2 & \\
\hline Figure $6 \mathrm{E} \mathrm{wt}$ & 2 & \\
\hline Figure $6 \mathrm{E}$ aptf- $1(-)$ & 2 & \\
\hline Figure S2A & 4 & \\
\hline Figure S2B & 4 & \\
\hline Figure S2C & 9 & \\
\hline Figure S2D & 9 & \\
\hline Figure S2E & 3 & \\
\hline Figure S2F & 3 & \\
\hline Figure S2G & 8 & \\
\hline Figure $\mathrm{S} 2 \mathrm{H}$ & 8 & \\
\hline Figure S2I & 8 & \\
\hline Figure S2J & 8 & \\
\hline Figure S2K & 5 & \\
\hline Figure S2L & 5 & \\
\hline Figure S2M & 9 & \\
\hline Figure S2N & 9 & \\
\hline Figure S3A with ATR & 2 & \\
\hline Figure S3A without ATR & 2 & \\
\hline Figure S3B with ATR & 2 & \\
\hline Figure S3B without ATR & 2 & \\
\hline Figure S3C, D with ATR & 4 & \\
\hline Figure S3C, D without ATR & 3 & \\
\hline Figure S3E, before RIS inhibition & 4 & \\
\hline Figure S3E, during RIS inhibition & 4 & \\
\hline Figure S3E, after RIS inhibition & 4 & \\
\hline Figure S4A wt & 2 & From Figure 4C \\
\hline Figure S4A rol-6(gf) & 2 & \\
\hline Figure S4A daf-2(-); daf-16(-) & 4 & $\begin{array}{l}\text { Reanalysis of DIC movies from Figure } \\
4 \mathrm{C}\end{array}$ \\
\hline Figure S4A daf-2(-); daf-16(-); Pdaf-16::daf-16+rol-6(gf) & 3 & \\
\hline Figure S4A daf-2(-); daf-16(-); Punc-119::daf-16+rol-6(gf) & 5 & \\
\hline Figure S4A daf-2(-); daf-16(-); Pmyo-3::daf-16+rol-6(gf) & 5 & \\
\hline Figure S4A daf-2(-); daf-16(-); Pges-1::daf-16+rol-6(gf) & 4 & \\
\hline Figure S4B rol-6(gf) & 2 & \\
\hline Figure S4B daf-16(-); aak-2(-);pgp-1::rfp+rol-6(gf) & 4 & \\
\hline 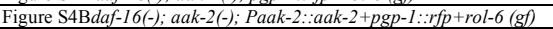 & 5 & \\
\hline Figure S4B daf-16(-); aak-2(-); Prgef-1::aak-2+pgp-1::rfp +rol-6 (gf) & 8 & \\
\hline Figure S4Bdaf-16(-); aak-2(-); Pmyo-3::aak-2+pgp-1::rfp +rol-6 (gf) & 5 & \\
\hline Figure S4B daf-16(-); aak-2(-); Ppgp-1::aak-2+pgp-1:rrfp+rol-6 (gf) & 5 & \\
\hline Figure S4B daf-16(-); aak-(-); Pdpy-7::aak-2+pgp-1::rfp+rol-6(gf) & 3 & \\
\hline Figure S4B daf-16(-); aak-2(-); Pdpy-7::aak-2+pgp-1:rrfp+rol-6 (gf) & 3 & \\
\hline Figure S4C rol-6 $(g f)$ & 2 & \\
\hline Figure S4C daf-16(-); aak-2(-); pgp-1::rfp+rol-6(gf) & 4 & \\
\hline Figure S4C daf-16(-); aak-2(-); Paak-2::aak-2+rol-6 (gf) & 3 & \\
\hline Figure S4C daf-16(-); aak-2(-); Psur-5::aak-2+rol-6(gf) & 3 & \\
\hline Figure S4C daf-16(-), a ak-2(-), Punc-119::aak-2+rol-6 (gf) & 2 & \\
\hline Figure S4C daf-16(-); aak-2(-); Pelt-2::aak-2+rol-6(gf) & 3 & \\
\hline Figure S4C daf-16(-); aak-2(-); Punc-54:aak-2+rol-6 (gf) & 3 & \\
\hline Figure S4C daf-16(-); aak-2(-); Psulp-5::aak-2+rol-6 (gf) & 3 & \\
\hline Figure S4C daf-16(-); aak-2(-); Pdpy-7::aak-2+Psur-5::GFP & 3 & \\
\hline Figure S5A, wt, adult feeding & 3 & \\
\hline Figure S5A, aptf-I(-), adult feeding & 3 & \\
\hline Figure S5B, wt, adult feeding & 2 & \\
\hline Figure S5B, RIS(-), adult feeding & 2 & \\
\hline Figure S5C, wt, adult starved & 2 & \\
\hline Figure S5C, aptf-1(-), adult starved & 2 & \\
\hline Figure S5D, wt, adult starved & 2 & \\
\hline Figure S5D, RIS(-), adult starved & 2 & \\
\hline Figure S5E, wt, arrested L1 starved & 3 & \\
\hline Figure S5E, aptf-1(-), arrested L1 starved & 3 & \\
\hline Figure S5F, wt, arrested L1 starved & 3 & \\
\hline Figure S5F, RIS(-), arrested L1 starved & 3 & \\
\hline Figure S5G, wt, arrested L1 starved & 3 & \\
\hline Figure S5G, aptf-1(-), arrested L1 starved & 3 & \\
\hline Figure $\mathrm{S} 5 \mathrm{H}, \mathrm{wt}$, arrested $\mathrm{L} 1$ & 3 & \\
\hline Figure S5H, RIS(-), arrested L1 & 3 & \\
\hline Figure S5I, daf-16(-); aak-1(-); aak-2(-), arrested L1 & 2 & \\
\hline Figure S5I, daf-16(-); aak-1(-); aak-2(-); aptf-1(-), arrested L1 & 2 & \\
\hline Figure S5J, wt, arrested L1 feeding & 2 & \\
\hline Figure S5J, aptf-I(-), arrested L1 feeding & 2 & \\
\hline Figure S5J, RIS(-), arrested L1 feeding & 2 & \\
\hline Figure S6A GPF OP50 control & 2 & \\
\hline Figure S6A GFP OP50 L1 arrested worm & 2 & \\
\hline Figure S6B reproductive growing L1 & 2 & \\
\hline Figure S6B arrested L1 & 2 & \\
\hline Figure S6C FUDR arrested L1, starved & 2 & \\
\hline Figure S6C FUDR arrested L1, feeding & 2 & \\
\hline
\end{tabular}

Table S6. Number of replicates for all experiments. Related to all Figures and Supplemental Figures. 
Table S7

\begin{tabular}{|c|c|}
\hline Primer name & Primer sequence 5 ' -3 ' \\
\hline \multicolumn{2}{|l|}{ aak-1(tm1944) } \\
\hline 890 & ACTGTTCCGAGTCCTTTCATTC \\
\hline 891 & TTCCCTTTCTTCGCTCACTTTT \\
\hline 892 & ATGGAACTGTGTATCGGCCA \\
\hline \multicolumn{2}{|l|}{ aak-2(ok524) } \\
\hline 893 & ATCGCCAAATTATGCTGCCC \\
\hline 894 & GGCAGGGTTCCACAAAGAAG \\
\hline 895 & AAGGAGACACTCGGAGTTGG \\
\hline \multicolumn{2}{|l|}{ aptf-1(ok974) } \\
\hline 48 & CGACAATCTTCCCAAAGACC \\
\hline 49 & CGGATCGATTGCTAGAGAGG \\
\hline 50 & GCTTGGACGGCTTTAGTTGA \\
\hline \multicolumn{2}{|l|}{$d a f-2(e 1370) *$} \\
\hline 519 & CGGGATGAGACTGTCAAGATTGGAGATTTCGG \\
\hline 520 & CAACACCTCATCATTACTCAAACCAATCCATG \\
\hline \multicolumn{2}{|l|}{ daf-16(mgDf50) } \\
\hline 636 & AGGTCAGAGTTCTAGGCGTAAA \\
\hline 637 & CGGTTCCAGCAATTCCAAGT \\
\hline 638 & AGTGGATTCTGAGCACACGA \\
\hline \multicolumn{2}{|l|}{ daf-16(mu86) } \\
\hline 468 & AAAGAGCTCGTGGTGGGTTA \\
\hline 469 & TCGCGCCTTTGTCTCTCTAT \\
\hline 470 & AGTGTCGAGTGAAGGGAGC \\
\hline \multicolumn{2}{|l|}{ sir-2.1(ok434) } \\
\hline 1107 & GTTTGATCACTCCTTCGCAAC \\
\hline 1108 & TCCCAGGACAGTTCGTACC \\
\hline 1109 & GCGGAAATGGCAGAAGTAGT \\
\hline \multicolumn{2}{|l|}{ daf-18(ok480) } \\
\hline 1090 & AGCATGGACTTCAACGATACAA \\
\hline 1091 & GAATGCCAGATTGCCGAACA \\
\hline 1092 & CGGTGGTCCATTTGAGATACC \\
\hline
\end{tabular}

Table S7. List of PCR primers used for Duplex PCR Genotyping. Related to STAR Methods. *The daf-2 mutant allele was identified using restriction with NcoI-HF (in CutSmart Buffer, New England Biolabs).

\section{Supplemental Reference}

S1. Narbonne, P., and Roy, R. (2009). Caenorhabditis elegans dauers need LKB1/AMPK to ration lipid reserves and ensure long-term survival. Nature 457, 210-214. 


\subsection{Sleep in adult $C$. elegans}

\subsubsection{Sleep is not required for adult longevity}

No evidence that sleep influences the lifespan of both starved and fed adults on the NGM plates was found in the previous study [118]. In order to investigate how living environments influence longevity though sleep, I measured the lifespan of adult worms in the M9 buffer with the presence or absence of food. No statistically significant difference was observed between wild-type worms and aptf-1 mutants during feeding or starvation. The RIS-ablated adult worms showed a reduced lifespan with food but not during starvation (Figure 1A and 1B). To know whether sleep affects the impact of diet on longevity, I tested the lifespan of adult worms with intermittent fasting. As was expected, sleep did not influence the life extension induced by diet (Figure 1C).

\subsubsection{Sleep does not affect adult lifespan expectancy caused by a metabolic alteration}

To further investigate the potential role of sleep in glucose metabolism, I tested the lifespan of adult worms that lived on NGM plates that contained $10 \mathrm{mM}$ 2-Deoxy-Dglucose, $100 \mathrm{mM}$ D-glucose and $0.1 \mathrm{mM}$ sodium azide respectively. 2-Deoxy-D-glucose is a glucose molecule that is unable to be metabolized. It blocks glycolysis and therefore leads to lifespan extension by inducing mitochondrial respiration [133, 134].

A high-glucose diet with D-glucose increases glucose availability and thus shortens the lifespan of worms [135]. Sodium azide is a metabolic inhibitor and has been used for anesthesia in C. elegans. Short exposure to sodium azide induces stress responses and reduces the lifespan of the L1 and L2 larvae of C. elegans [136]. However, no evidence was found to show that sleep is involved in the glucose or energy metabolism for regulating longevity in adult C. elegans (Figure 1D-1F). 


\subsubsection{Sleep is dispensable for TOR and FoxO signaling pathway for regulating lifespan of adults}

To test the putative role of sleep in the TOR signaling pathway regarding longevity, I used RNAi to knock down the TOR signaling-related genes let-363, pha-4 and skn-1. In the control groups that fed with RNAi GFP, no constant significant reduction of lifespan in the aptf-1 mutant was found compared to the wild-type adult (Figure 1G-1I). Knockdown of let-363 did not alter the lifespan in wild-type adults, but the aptf-1 mutants with let-363 RNAi showed a slight lifespan decrease compared to the wild-type worms with let-363 RNAi (Figure 1G). However, there was no difference observed in the lifespans of aptf-1 mutants and wild-type adults in pha-4 RNAi, nor in skn-1 RNAi assays (Figure 1H, 1I).

I then questioned whether sleep might influence the lifespan expectancy that is regulated by the insulin-signaling pathway. To answerer this question, I crossed the aptf- 1 mutant with the daf-2 mutant or the daf-16 mutant respectively, and tested the adult lifespan of the double mutants in the presence or absence of food. Consistent with the literature, daf-2 mutant showed a dramatic increase in lifespan during feeding, while the daf-16 mutant showed a reduction in lifespan, whether with food or without. However, sleep did not seem to have any impact on those lifespan expectancies (Figure 1J-1L). Taken together, the results suggest that sleeplessness did not affect adult longevity, neither with the presence of food nor under starvation. In adult worms, sleep thus seems to be inessential for a normal adult lifespan. 
Figure 1

A

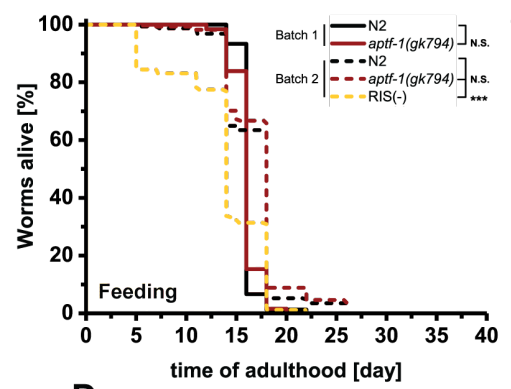

D

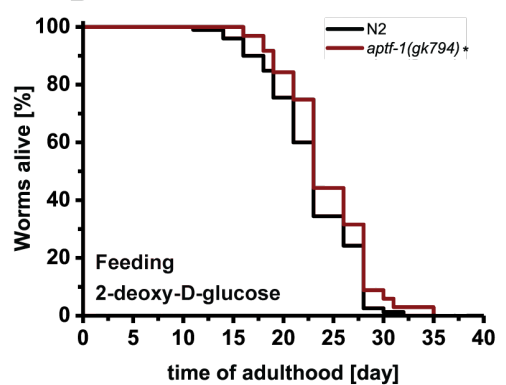

G

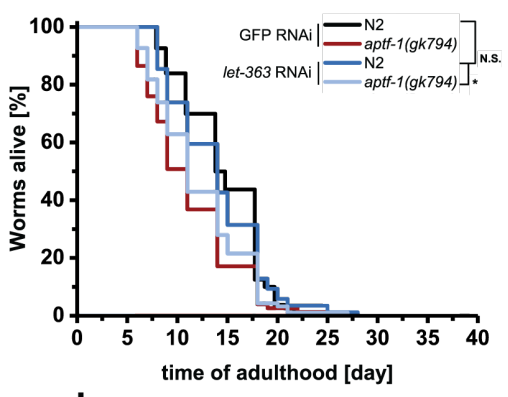

J

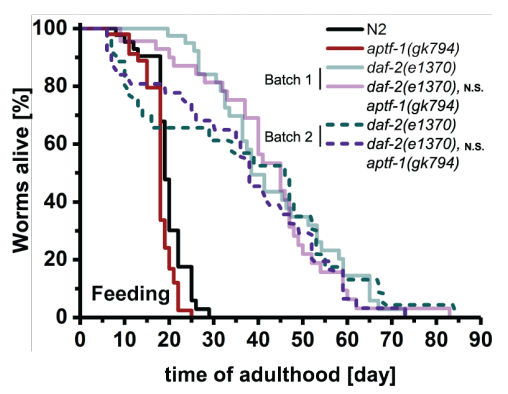

B

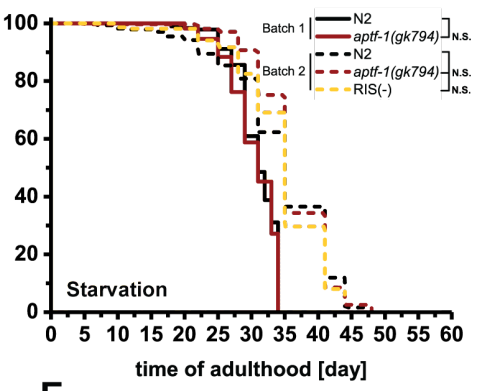

E

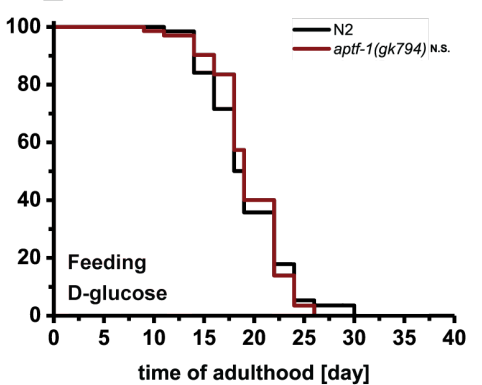

H

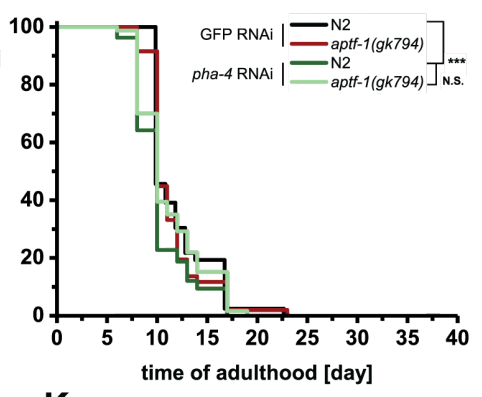

K

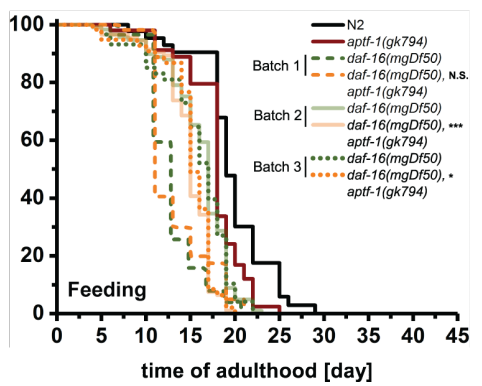

C

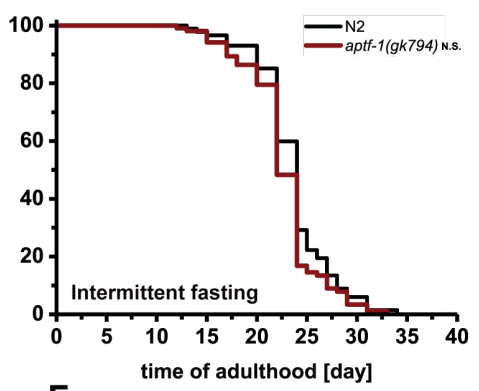

$\mathbf{F}$

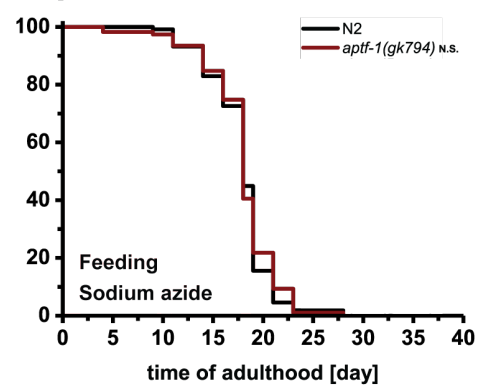

I

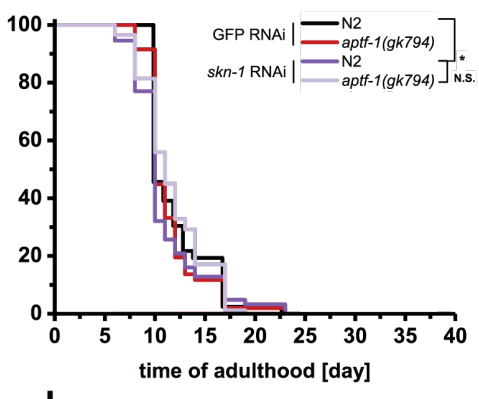

L

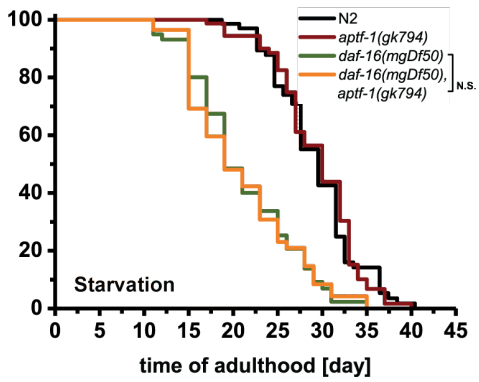

Figure 1 Sleep does not affect the lifespan of the adult worms.

(A) Lifespan of feeding adult in M9 buffer. Wild-type is not significantly different to aptf-1(gk794) but different to RIS (-) (yellow). RIS (-) adults live shorter than wild-type adults $(-18.4 \%), * * *$ p $<0.001$. (B) Lifespan of the starved adult in M9 buffer. No significant difference was found between wild-type, aptf1 (gk794) and RIS(-) (yellow). (C) Lifespan of adults by intermittent fasting. No significant difference between groups was present. (D) Lifespan of feeding adult with $10 \mathrm{mM}$ 2-deoxy-D-glucose. The aptf1 (gk794) mutant live slightly longer than wild-type adults $(+6.6 \%),{ }^{*}<0.05$. (E) Lifespan of feeding adult with $100 \mathrm{mM}$ D-glucose. No significant difference between groups was present. (F) Lifespan of feeding adult with $0.1 \mathrm{mM}$ sodium azide. No significant difference between groups was shown. (G-I) Feeding adult lifespan of let-363 RNAi (G), pha-4 RNAi (H), and skn-1 RNAi (I). Control group was fed with GFP RNAi. The adult worms let-363 RNAi; aptf-1 (gk794) lived shorter than let-363 RNAi (-12.9\%), $* \mathrm{p}<0.05$. No significant difference was found between 1. The adult worms $s k n-1$ RNAi and $s k n-1$ RNAi/aptf-1 (gk794) and 2. The adult worms pha-4 RNAi and pha-4 RNAi/aptf-1(gk794). (J) Feeding adults daf-2 (e1370); aptf-1 (gk794) lived as long as the feeding adult daf-2(el370). Replicates were shown 
in different batches. (K) Feeding adult daf-16(mgDf50)/aptf-1(gk794) double mutants showed reduced lifespan when compared to daf-16(mgDf50). The decline was not consistently significant in the replicates. (L) No significant difference in lifespan was shown in starved adults between daf-16(mgDf50) and daf16(mgDf50); aptf-1 (gk794) double mutants. Biological replicates were shown as different batches by different lines and symbols. Comparisons between two groups of all the lifespans were made using the log-rank test. The details of all the lifespans can be found in table S1.

\subsection{Sleep plays a central role in starvation-survival of arrested L1 $C$. elegans}

In contrast to its dispensable role in adult longevity, sleep becomes indispensable in the survival of arrested L1 larvae and serves as a predictor for survival time during starvation. It has been previously shown that the L1-arrested aptf-1 mutant displayed a shorter survival span than the wild-type L1 larvae in M9 buffer [118]. To exclude the possibility that the reduction in survival of the aptf-1 mutant is caused by the living environment rather than sleep deprivation, I first tested the survival of the arrested $f l p-11$ L1 mutant in M9 buffer and the aptf-1 mutant on NGM plates. As expected, both of them showed a significant decrease in survival compared to the wild-type L1 larvae (Figure 2A, 2B). This suggested that sleep is mainly responsible for the starvationsurvival of starved L1 larvae independent of the living environment.

Additionally, I also quantified the upper-limit of bacteria consumption for keeping the freshly hatched larvae in L1 arrested stage (Figure S1). This will contribute to a better assay performance in future investigations.

\subsection{Metabolic alteration is involved in the sleep-protective program during starvation.}

In order to understand the underlying mechanisms of protective program of sleep, I next investigated the survival of starved L1 larvae with different chemical inhibitors. Cycloheximide inhibits the translocation step in protein synthesis. Addition of cycloheximide $(0.1$ to $10 \mu \mathrm{M})$ decreased the protein levels and increased the longevity in adult C. elegans [137]. However, cycloheximide with low concentration only interferes in the cytoplasmic protein synthesis, not in mitochondrial protein synthesis [138]. To evaluate the link between sleep and protein synthesis, I exposed the L1 larvae in M9 
buffer with highly-concentrated cycloheximide (1 to $2 \mathrm{mM}$ ) to block the protein synthesis completely. As shown in figure $2 \mathrm{C}$, cycloheximide dramatically reduced survival of the starved L1 wild-type larvae as well as the aptf-1 mutant larvae. However, aptf-1 mutants still showed significant decreased survival compared to the wild-type worms with cycloheximide.

Moreover, the addition of amino acid methionine did not consistently reduce survival of the arrested wild-type larvae and the aptf-1 larvae in the absence of food (Figure 2D). Taken together, the results suggest that protein synthesis is not involved in the early death of the sleepless L1 mutants during starvation.

Furthermore, to test whether metabolism is involved in the protective program, I tested the survival of arrested L1 with $1 \mu \mathrm{M}$ sodium azide. Interestingly, sodium azide at the low concentration decreases the survival of arrested wild-type L1 larvae but not the survival of the sleepless aptf-1 mutants (Figure 2E). This indicates that the inhibition of metabolism might be relevant to the mechanism of the sleep-protective program in survival.

Figure 2

A

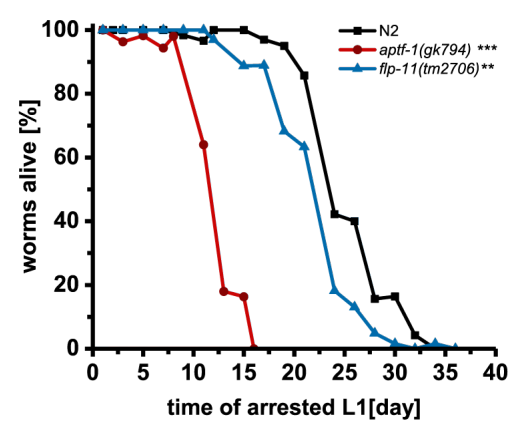

D

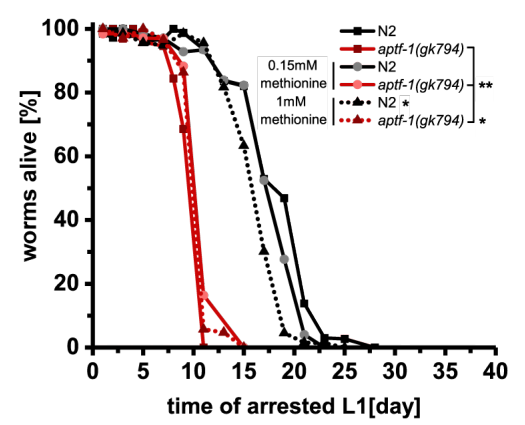

B

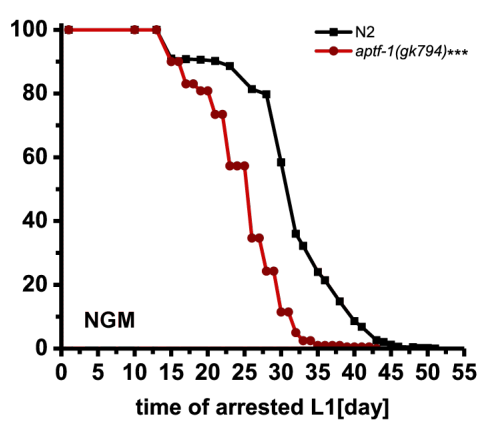

E

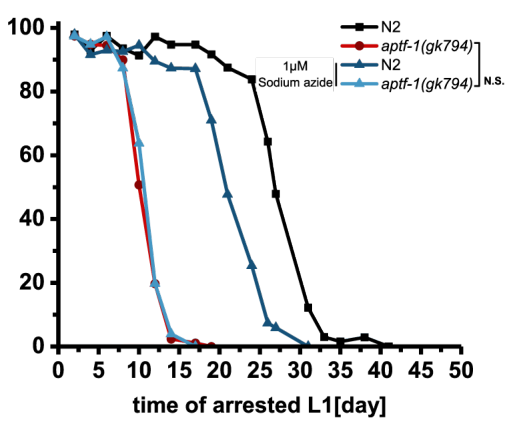

C

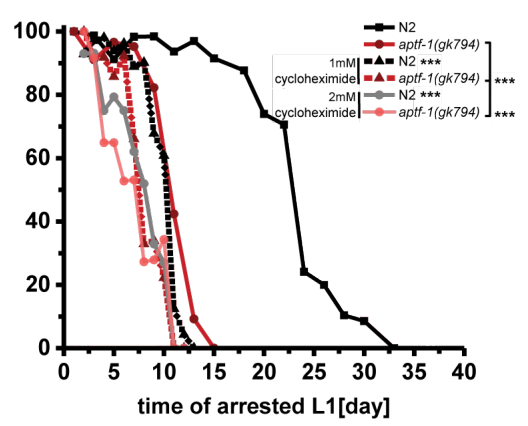

Figure 2 Sleep is required for survival of arrested L1 larvae during starvation. 
(A) The arrested L1 mutants flp-11(tm2706) survived longer than the aptf-1(gk794) mutant larvae but shorter than the wild-type larvae $(-6.7 \%),{ }^{* *} \mathrm{p}<0.01$. (B) Arrested L1 survival span of aptf-1 $(g k 794)$ mutants on NGM plates, aptf-1(gk794) larvae survived shorter than wild-type L1 larvae (-22.1\%), $* * * p<0.001$. (C) Arrested L1 survival in the M9 buffer with $1 \mathrm{mM}$ or $2 \mathrm{mM}$ cycloheximide. Cycloheximide significantly reduced the survival of both wild-type and aptf-1(gk794) arrested L1 worms. Details see table S2. (D) Methionine slightly increased the survival of aptf-1 ( $g k 794)$ mutants, ${ }^{* *} \mathrm{p}<0.01$ with $0.15 \mathrm{mM}$ methionine, ${ }^{*} \mathrm{p}<0.05$ with $1 \mathrm{mM}$ methionine. Details see table S2. (E) Arrested L1 larvae survival span in the M9 buffer with $1 \mu \mathrm{M}$ sodium azide. Sodium azide reduced the survival of wild-type L1 larvae $(-22.7 \%, * * * \mathrm{p}<0.001)$ but not that of aptf-1(gk794) mutants. The wild-type (N2) worms were shown in the black line, aptf-1 (gk794) mutant worms were shown in red line. Treated worms or mutants as well as the biological replicates were shown in each figure with different colors and symbols. To compare the $50 \%$ survival point of two lifespan groups, a Fischer's exact test was performed. Details of the survival span are in table S2.

\subsection{The decrease of survival in the sleepless mutant is recovered by ethanol}

I next aimed to find out whether TOR signaling participates in the protective sleep program and contributes to survival in arrested L1 during starvation. I thus used rapamycin, an inhibitor of TOR signaling. It was reported in a previous study that rapamycin extends the lifespan of adult $C$. elegans by inhibiting both TORC1 and TORC2 [139]. Because of the poor solubility of rapamycin in only $0.1 \%$ DMSO, I added $0.1 \%$ ethanol to increase the solubility. Surprisingly, rapamycin dissolved in $0.1 \%$ DMSO, and $0.1 \%$ ethanol can mostly rescue the decline of survival in sleep-deprived aptf-1 L1 mutants during starvation (Figure 3A). However, the control group with only $0.1 \%$ DMSO, and $0.1 \%$ ethanol showed the same rescue as the assay with rapamycin (Figure 3B). I thus tested the survival of L1 larvae with either $0.1 \%$ DMSO or $0.1 \%$ ethanol respectively (Figure 3C, 3D). As shown in figure 3D, the survival decline in the sleepless mutants aptf-1 can be rescued by adding $0.1 \%$ ethanol. This indicates that the effect of rescue for survival in fact came from the ethanol. Interestingly, $0.1 \%$ ethanol did not increase the survival time of wild-type L1 larvae during starvation. These observations suggest that instead of rapamycin, the metabolism of ethanol might have a potential common mechanism underlying the protective program of sleep in starvation survival.

To further understand how ethanol affects larvae survival through the protective program of sleep during starvation, I thus exposed the arrested L1 larvae to $0.1 \%$ ethanol and analyzed their sleep factions. As shown in figure $3 \mathrm{E}$, aptf-1 mutant larvae displayed a 
weak increase of sleep after the addition of ethanol. However, the arrested wild-type L1 larvae with $0.1 \%$ ethanol did not sleep more than the worms without ethanol. This indicates that ethanol might act in the downstream of sleep pathway to regulate survival but not behavior during L1 starvation.

Lastly, I wondered whether ethanol could further influence the ability of starved larval worms for re-entering development after feeding. Surprisingly, I found that ethanol destroyed this re-develop ability of the long-starved L1 larvae. The longer the larva starves with ethanol, the tougher it returns to the developmental stages when fed (Figure $3 \mathrm{~F})$. The results suggest that the metabolism of ethanol is involved in the developmental mechanisms and can counteract the decline of survival caused by sleep deprivation at the expense of the re-developmental ability in larval C. elegans. 
Figure 3

A

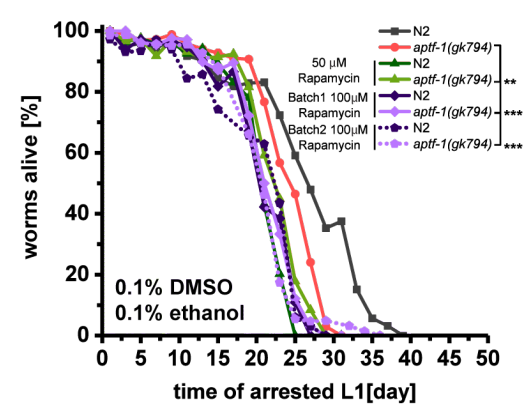

D

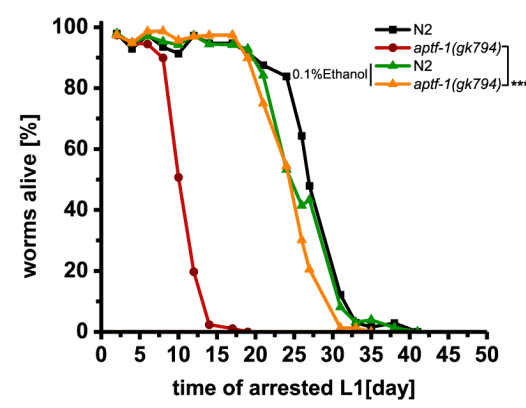

B

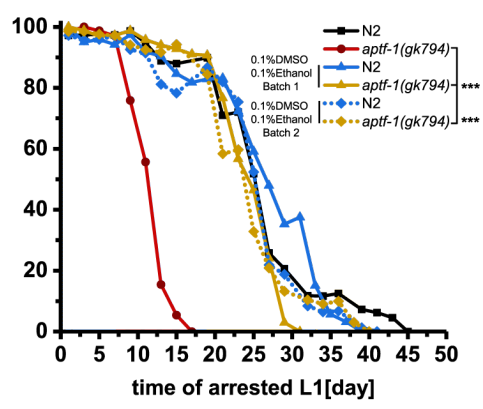

$E_{\text {Arrested L1 larvae, starved with ethanol }}$

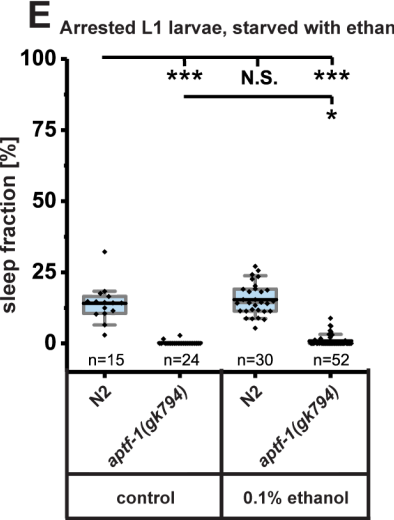

$\mathbf{F}$

Percentage of development, arrested L1 larvae with $0.1 \%$ ethanol

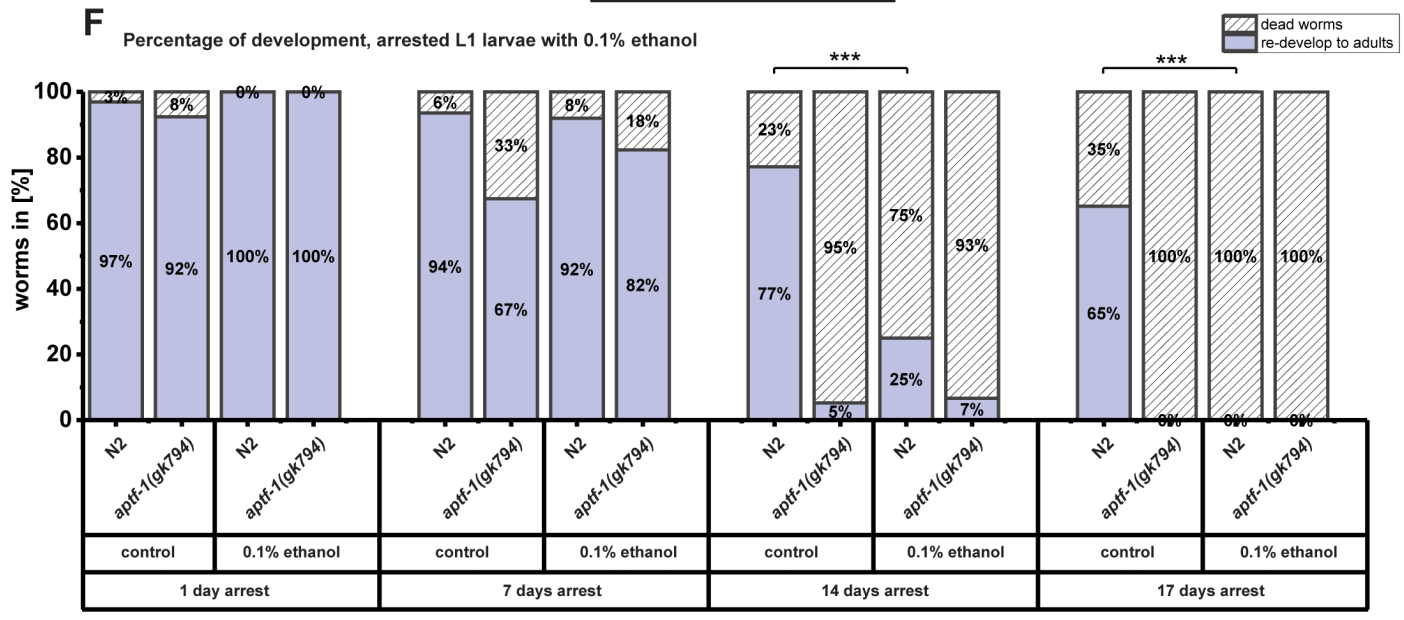

Figure 3 Instead of rapamycin, ethanol acts a potential role in the sleep mechanism for extension of survival in arrested L1 larvae.

(A) Rapamycin is harmful for survival of the arrested L1 larvae. Rapamycin was dissolved in M9 buffer with $0.1 \%$ DMSO and $0.1 \%$ ethanol as background. The control group was treated with only $0.1 \%$ DMSO and $0.1 \%$ ethanol in M9 buffer. The aptf-1(gk794) larvae with rapamycin at both concentrations showed a decreased survival than the control aptf-1(gk794): $50 \mathrm{mM}$ rapamycin, $-8.2 \%, * * \mathrm{p}<0.01 ; 100 \mathrm{mM}$ rapamycin batch $1,-13.6 \%, * * * p<0.001 ; 100 \mathrm{mM}$ rapamycin batch $2,-13.1 \%, * * * \mathrm{p}<0.001$. (B) Survival of the aptf-1(gk794) mutant L1 larvae was increased by adding $0.1 \%$ DMSO and $0.1 \%$ ethanol, $* * * p<0.001$ in both batches. (C) $0.1 \%$ DMSO didn't affect survival of arrested L1 larvae. (D) $0.1 \%$ ethanol rescued the decline of survival of aptf-1 $(g k 794)$ mutant larvae $(+142.8 \%), * * * p<0.001$. Details of the survival span are in table S2. (E) Ethanol did not increase sleep of the wild-type L1 larvae but can slightly induce sleep (in mean value but not median value) of aptf-1(gk794) mutants during starvation, $*_{p}<0.05$. Individual worm was shown in the box plot as dots, median sleep fraction: N2 control $14.3 \%$; aptf-1 (gk794) mutant 0\%; N2 ethanol: 14.4\%; aptf-1(gk794) mutant 0\%. (F) Arrested L1 larvae with the ethanol treatment showed a significant decline in the ability to re-enter development when fed. The first day of late-L4 stage was scored for successful development. 1 day arrested larvae: control N2: $n=65$, 
recover to L4 stage (RCto4): 63; control aptf-1 (gk794): n=79, n(RCto4)=73; N2 with 0.1\%ethanol: $n=53$, $\mathrm{n}(\mathrm{RCto})=53$; aptf-1 (gk794) with $0.1 \%$ ethanol: $\mathrm{n}=50, \mathrm{n}(\mathrm{RCto} 4)=50 ; 7$ days arrested larvae: control $\mathrm{N} 2$ : $\mathrm{n}=78, \mathrm{n}(\mathrm{RCto})=73$; control aptf-1(gk794): $\mathrm{n}=86, \mathrm{n}(\mathrm{RCto} 4)=58 ; \mathrm{N} 2$ with $0.1 \%$ ethanol: $\mathrm{n}=50$, $\mathrm{n}(\mathrm{RCto} 4)=46$; aptf-1 $(\mathrm{gk} 794)$ with $0.1 \%$ ethanol: $\mathrm{n}=51, \mathrm{n}(\mathrm{RCto} 4)=42 ; 14$ days arrested larvae: control N2: $\mathrm{n}=79, \mathrm{n}(\mathrm{RCto} 4)=61$; control aptf-1 $(g k 794): \mathrm{n}=76, \mathrm{n}(\mathrm{RCto} 4)=4$; N2 with $0.1 \%$ ethanol: $\mathrm{n}=72$, $\mathrm{n}(\mathrm{RCto} 4)=18, * * * \mathrm{p}<0.001$ compared to $\mathrm{N} 2$ control; aptf-1 $(g k 794)$ with $0.1 \%$ ethanol: $\mathrm{n}=75, \mathrm{n}(\mathrm{RCto} 4)=5$; 17 days arrested larvae: control $\mathrm{N} 2: \mathrm{n}=66, \mathrm{n}(\mathrm{RCto} 4)=43$; control aptf- 1 (gk794): $\mathrm{n}=60, \mathrm{n}(\mathrm{RCto})=0$; N2 with $0.1 \%$ ethanol: $\mathrm{n}=69, \mathrm{n}(\mathrm{RCto} 4)=0, * * * \mathrm{p}<0.001$ compared to $\mathrm{N} 2$ control; aptf -1 (gk 794$)$ with $0.1 \%$ ethanol: $\mathrm{n}=77, \mathrm{n}(\mathrm{RC}$ to 4$)=0$.

\subsection{Over-expression of FLP-11 does not benefit the survival in arrested L1 larvae}

Lack of the neuropeptide FLP-11 in RIS reduces the survival time in arrested L1 during starvation (see Figure 2A above). I wondered whether over-expression of FLP-11 might be able to increase sleep, and therefore prove beneficial to the survival of L1 larvae during starvation. I used the heat shock promote phsp-16.2 to increase the FLP-11 expression in L1 larvae, which starved in the M9 buffer, a M9 agar-chamber or a S-basal agar-chamber. In order to minimize the damage of the heat shock in C. elegans, the temperature was set to $25^{\circ} \mathrm{C}$. As shown in figure $4 \mathrm{~A}$, in $\mathrm{M} 9$ buffer, raising the FLP-11 expression level in starved L1 larvae is not able to really increase sleep. Also, in chambers, FLP-11 over-expression did not prolong the survival of starved L1 larvae at all (Figure 4B, 4C). However, it is necessary to note that I did not measure the expression level of FLP-11 after heat shock. Therefore, the fact that the heat-shock promoter phsp-16.2 might not be well activated at $25^{\circ} \mathrm{C}$ cannot be excluded. I also tested sleep fraction after heat shock in the starved L1 larvae. At $25^{\circ} \mathrm{C}$, over-expressing FLP11 slightly increased sleep (Figure 4D). However, the influence of temperature for sleep fraction in the arrested L1 larvae is still unknown. The increase of temperature seems to able to inhibit sleep in larvae during starvation, arrested L1 larvae sleep for $18 \%$ of the total time at $20^{\circ} \mathrm{C}$, but sleep for only $14 \%$ at $25^{\circ} \mathrm{C}$. Future study would be required to confirm the over-expression level of FLP-11 from the heat shock in the L1 larval worms. 
Figure 4

A

B

C
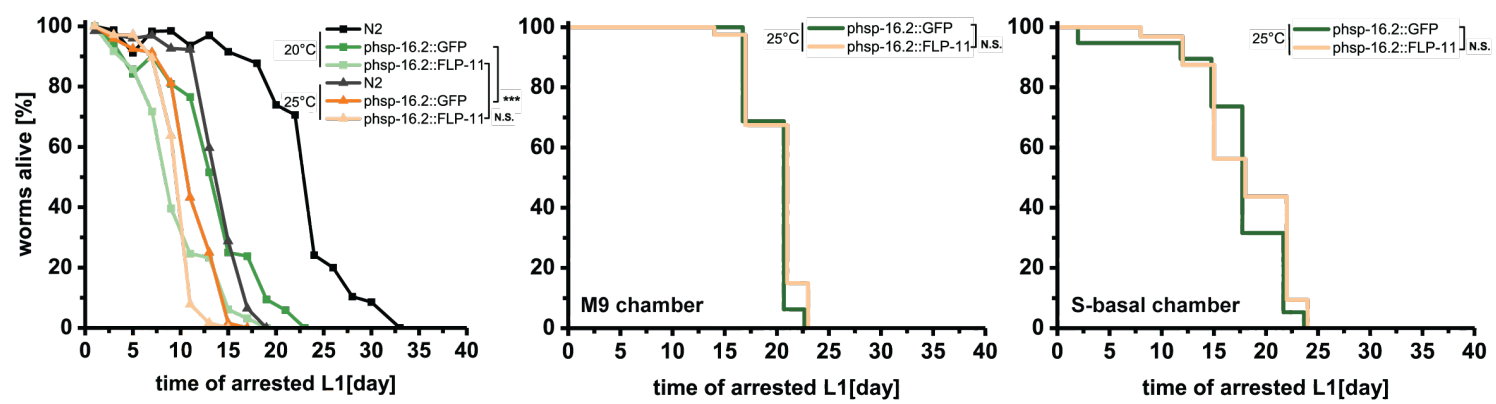

D

Arrested L1 larvae, starved

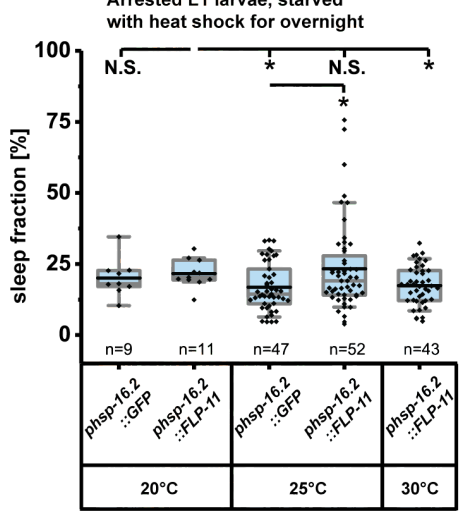

Figure 4 Over-expression of FLP-11 in arrested L1 larvae during starvation.

(A) Survival span of arrested L1 larvae in M9 buffer. Over-expression of FLP-11 didn't increase larval survival during starvation. Control group: at $20^{\circ} \mathrm{C}$; experimental group: at $25^{\circ} \mathrm{C}$. (B) Survival span of arrested L1 larvae in M9 chamber for over-expression of FLP-11, no significance was found between the FLP-11 over-expression group and the control group. (C) Survival span of arrested L1 larvae in S-basal chamber for over-expression of FLP-11, no significance was found. Survival of larvae at $20^{\circ} \mathrm{C}$ were taken as control. Treatments were shown in each figure. The Fischer's exact test was performed in comparisons of the 50\% survival point in arrested L1 larvae in M9 buffer. The log-rank test was used for comparisons between two survival spans in chamber. Details of all the lifespans can be found in table S2. (D) Sleep fraction after over-expressing FLP-11 in arrested L1 larvae during starvation. A slightly increase of sleep was found in arrested L1 larvae with FLP-11 over-expression compared with the control larvae at $25^{\circ} \mathrm{C}$, $* \mathrm{p}<0.05$. Individual worm was shown in the box plot as dots, median sleep fraction: phsp-16.2::GFP control $20^{\circ} \mathrm{C} 18.1 \%$; phsp-16.2::FLP-11 control $20^{\circ} \mathrm{C}, 20 \%$; phsp-16.2::GFP over-expression $25^{\circ} \mathrm{C}$, $14.4 \%$; phsp-16.2::FLP-11 over-expression $25^{\circ} \mathrm{C}, 19.1 \%$. Details of the survival span are in table S2.

\subsection{AMPK and PTEN are further involved in the protective program of sleep in arrested L1 larvae}

In the publication above, Masurat and I identified the upstream of the sleep-activate and sleep-promoting pathway in the presence and absence of food. AAK-1/2 and DAF-16 act in parallel to activate sleep during starvation. Tumor suppressor DAF-18 and sirtuin 
signaling with SIR-2.1 are involved in this pathway, and play a regulative role in the upstream of AMPK and FoxO in C. elegans [118]. In addition to the research stated above, I also tested the sleep fraction in L1 larvae of daf-2 mutants and daf-2/aptf-1 double mutants in different allele, sir-2.2 mutants in two alleles and sir-2.4 mutants during starvation. Data is shown in the supplemental figure S2A. Neither DAF-2 nor SIR-2.2 or SIR-2.4 can induce sleep in starved L1 larvae alone. Additionally, a sleep faction screen of different mutants during L1 starvation is shown in the figure S2B and $\mathrm{S} 2 \mathrm{C}$ as supplemental information.

To further know whether survival in arrested L1 larvae could be influenced by lacking one or two of these sleep-promotion genes, I investigated the survival span in different mutants. If the gene plays a role in the protective survival program induced by sleep, the survival time of starved L1 larvae should be shortened by a removal of the gene. Moreover, the decline in survival should not be further increased by the removal of the aptf-1 gene. To verify this hypothesis, I continued to test the lifespan of the aptf-1 mutant in the background of daf-16, daf-18, aak-2, sir-2.1 single mutants, or in the aak1/aak-2 double mutants respectively. Indeed, all of the single mutants showed a dramatic decline in survival during L1 larvae (Figure 5A, 5C-5E). Also, a decline of the ability to re-enter development when fed was shown in the daf-16(mgDf50)/aptf-1(gk794) double mutant compared to the aptf-1(gk794) mutant L1 larvae (Figure 5B). No consistent significant difference in survival was observed between daf-18 mutants and daf-18/aptf1 double mutants (Figure 5C), between aak-2 mutants and aak-2/aptf-1 double mutants, as well as between aakl/aak2 double mutants and aak1/aak2/aptf-1 triple mutants (Figure 5D). However, the daf-16/aptf-1 and sir-2.1/aptf-1 double mutants showed an apparent decrease in survival compared to $d a f-16$ or sir-2.1 single mutants respectively (Figure 5A, 5E).

Because daf-18 mutants have shown a complete loss of sleep in L1 starvation [118], I questioned which tissues PTEN functions contained to induce sleep during starvation. I thus expressed DAF-18 in the whole worm, muscle, intestine or hypodermis respectively in daf-18 L1 mutant larvae and then quantified their sleep fractions during starvation. The decline of sleep in the $d a f-18$ mutants can be fully rescued by expressing DAF-18 in the whole worm. Expressing DAF-18 in intestines or hypodermis but not in muscles also 
increased sleep in the daf-18 mutants (Figure S3). DAF-18 thus seems to act in the muscle and hypodermis to activate sleep in the starved L1 larvae.

All of these observations suggest that AMPK and PTEN are likely involved in a common mechanism with sleep in both sleep-active/sleep-promoting pathways and protective program in special tissues to improve larval survival during starvation, while the FoxO and sirtuin seem only to act in the upstream of sleep-active/sleep-promoting pathway.

\subsection{Sleep is related to RNA metabolic process in L1 larvae during starvation}

I also tested the survival of tut-1 mutants and tut-1/aptf-1 double mutants to investigate the potential role of RNA metabolic processes in L1 sleep and L1 survival during starvation. The tut-1 L1 mutants showed a significantly decreased survival time compared to wild-type L1 larvae during starvation. No difference was found between the survival of aptf-1 L1 larvae and that of tut-1/aptf-1 L1 larvae during starvation (Figure 5F). These results indicate that RNA metabolic processes might take part in the protective mechanism with sleep for survival extension in the absence of food. 
Figure 5

A

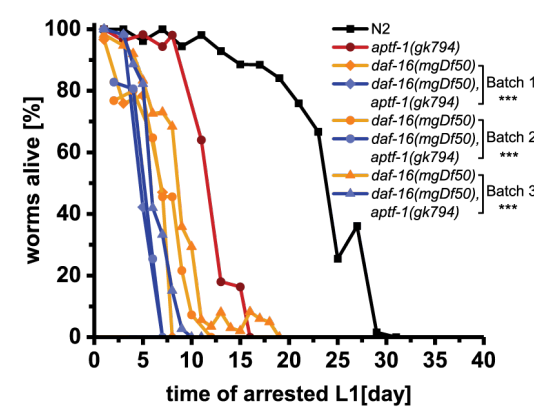

D

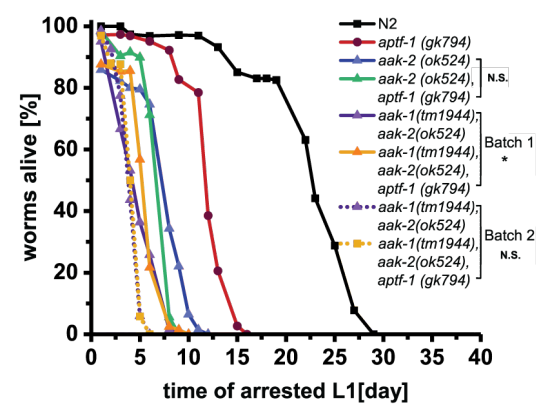

B

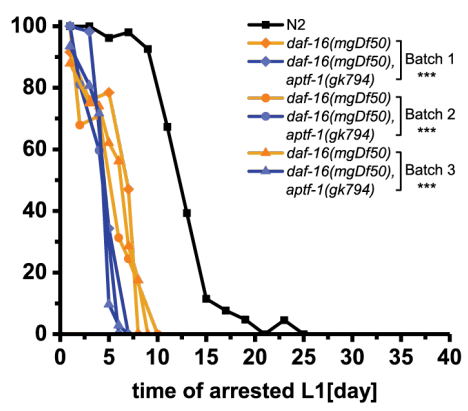

E

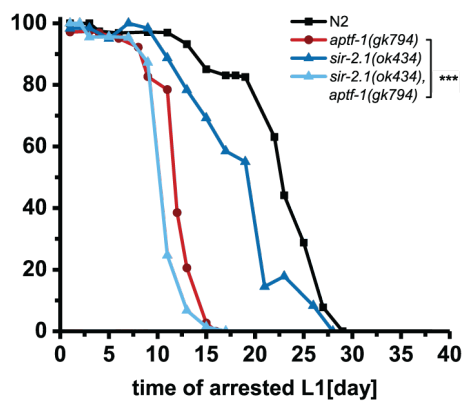

C

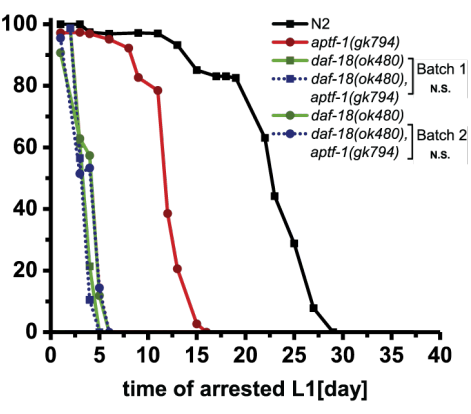

$\mathbf{F}$

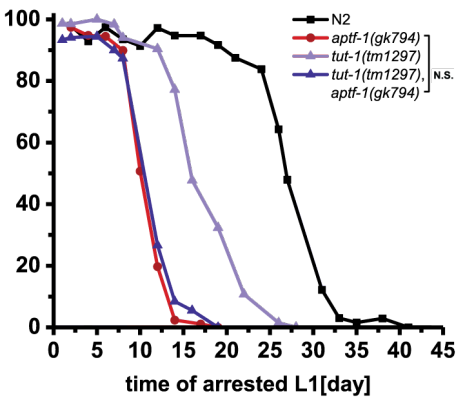

Figure 5 Mutants of longevity pathway are involved in the sleep mechanism for extension of survival in the L1 larvae during starvation.

(A) Arrested L1 daf-16(mgDf50)/aptf-1 (gk794) double mutants lived for a shorter time than the daf16(mgDf50) mutants. The decline was consistently significant in all the replicates (show in different batch), $* * * \mathrm{p}<0.001$. (B) Arrested L1 daf-16(mgDf50)/aptf-1 (gk794) double mutant showed a consistent significant decline in the ability to re-enter development when fed in all the replicates. $* * * p<0.001$. (C) Arrested L1 daf-18 (ok480)/aptf-1 (gk794) double mutants showed no reduced lifespan compared to daf-18 (ok480) mutants. (D) Arrested L1 aak-2(ok524)/aptf-1(gk794) double mutants lived for a shorter time than the aak-2(ok524) mutants, $* * * \mathrm{p}<0.001$. No significant difference was found between the arrested L1 aak2(ok524)/aak-1(tm 1944)/aptf-1(gk794) triple mutants and the aak-2(ok524)/aak-1(tm1944) double mutants. (E) Arrested L1 sir-2.1(ok434)/aptf-1(gk794) double mutants lived for a shorter time than the aptf-1 (gk794) mutants, ${ }^{* * *}<<0.001$. (F) Arrested L1 tut-1 (tm 1297) lived for a shorter length of time than wild-type L1 larvae. No significant difference was shown between the double mutant tut-1(tm 1297)/aptf$1(g k 794)$ and the mutants tut-1(tm 1297) of arrested L1 larvae. Wild-type (N2) was shown in black lines and aptf-1(gk794) in red lines. Mutants were shown in each figure with different colors and symbols. Biological replicates were shown as different batches by different lines and symbols. To compare the $50 \%$ survival point of two groups, the Fischer's exact test was used. Details of the survival spans can be found in table S3. Details of the re-enter development data are shown in table S4.

\subsection{TOR signaling is involved in the protective sleep program during starvation}

TOR is a nutrient sensor. It regulates metabolic mechanisms during dietary restriction or starvation. Together with IIS signaling, TOR plays a crucial role in the nutrient-sensing network on the lifespan regulation [140]. Because IIS singling has been proven to be necessary in the sleep-promoting pathway of L1 larvae during starvation [118], I 
questioned whether TOR signaling is also involved in this program. In my previous work, I have tested the survival rates in starved L1 larvae with the chemical inhibitor rapamycin (see Figure 3A above). However, since the assay was limited by the solubility of rapamycin and the unexpected effects from ethanol (see Figure 3D above), it was not possible to address the primary role of TOR signaling in sleep and survival by adding rapamycin.

For this reason, I turned my focus to the RICT-1/Rictor and the GTPase RAGA-1, which are co-activators of TORC2 and TORC1 respectively [141-143]. Previous studies showed that the inhibition of RICT-1/Rictor or RAGA-1 extends lifespans in adult $C$. elegans [143-145]. As shown in figures 6A and 6B, no consistent significant increase in survival was found in either rict-1 mutants or raga-1 mutants when compared to wildtype L1 larvae during starvation. Interestingly, both rict-1/aptf-1 and raga-1/aptf-1 double mutants survived significantly longer than the aptf-1 mutants with the absence of food. Therefore, TOR signaling seems to play a cooperative role in the protective sleep program.

Figure 6

A

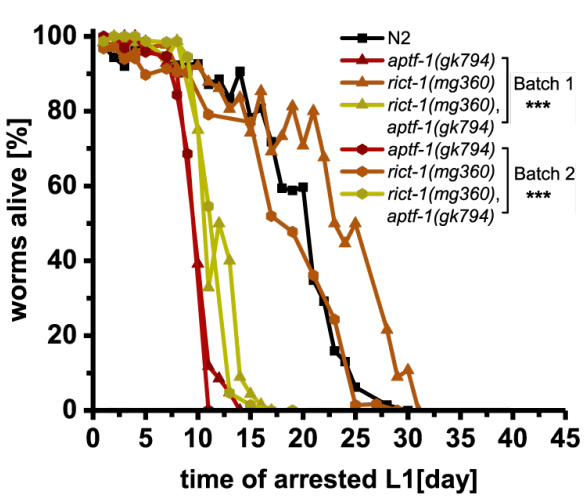

B

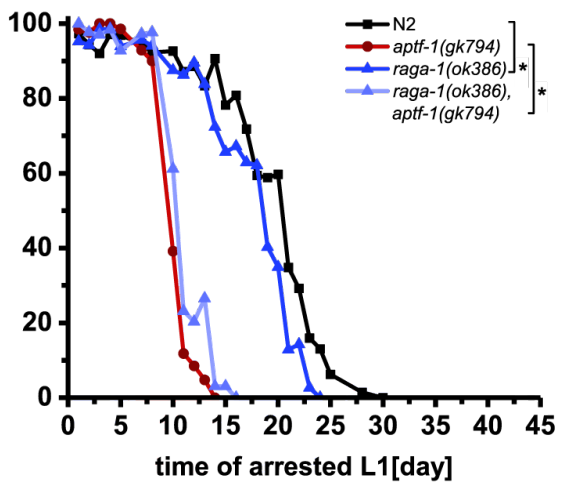

Figure 6 TOR signaling is involved in the protective sleep program during starvation.

(A) Arrested L1 double mutant rict-1 (mg360)/aptf-1(gk794) lived for significantly longer than aptf1 (gk794) in two replicates. Both batches, ${ }^{* *} \mathrm{p}<0.001$. (B) Arrested L1 rage-1(ok386)/aptf-1(gk794) lived for slightly longer than rage-1(ok386), $* \mathrm{p}<0.05$. Wild-type $(\mathrm{N} 2)$ is shown in a black line and aptf1 ( $g k 794)$ is shown in a red line. Mutants are shown in each figure, with different colors and symbols. To compare the $50 \%$ survival point of two lifespan groups, a Fischer's exact test was performed. Details of the survival span are in table S5. 


\subsection{Apoptotic cell death and autophagy are involved in the protective program of sleep}

Since the protective program of sleep seems to interact with multiple physiological mechanisms during starvation, I aimed to establish which process is involved in this survival strategy. CEP-1 (C. elegans P-53-like protein) is an ortholog of the tumor suppressor protein p53 [146]. It is induced by DNA damage and then further active in the apoptotic cell death in germline by increasing the EGL-1 and CED-13 expressions [147, 148]. EFK-1 (Elongation Factor Kinase) is an ortholog of the elongation factor 2 kinase $(\mathrm{eEF} 2 \mathrm{~K}$ ) that protect cells during nutrient starvation [149]. ATG-18 is an autophagy protein. It serves in lifespan-maintaining in response to dietary restriction by regulating neuropeptide signals [150].

As shown in figure 7A, the cep-1 L1 mutants survived better than the wild-type L1 larvae during starvation. Interestingly, this survival advantage was removed entirely by sleep deprivation in cep-l/aptf-1 double mutants. Moreover, cep-1 mutant did not show decreased sleep during L1 starvation (Figure S2A). All the results suggest that CEP-1 acts in the downstream pathway of sleep for promoting survival during starvation. Single mutant efk-1 displayed a decreased survival rate when compared to the wild-type L1 larvae. The same reduction in survival rate can also be found in efk-1/aptf-1 double mutants (Figure 7B). It indicates that EFK-1 and sleep act in two distinct pathways to promote survival in starved L1 larvae. No difference was found between atg-18 mutants and atg-18/aptf-1 double mutants (Figure 7C). Both of the mutants showed the severe reduction of survival during starvation, suggesting that ATG-1 and sleep have a common pathway in promoting survival in starved L1 larvae.

The metr-1 gene encodes methionine synthase in C. elegans. Lack of metr-1 did not extend the lifespan of adult worms [151]. The metr-1 L1 mutants showed a shorter survival rate than wild-type L1 larvae during starvation (Figure 7D). However, to further understand the relationship between sleep and methionine metabolism, survival data from metr-1/aptf-1 double mutants is required. 
Figure 7

A

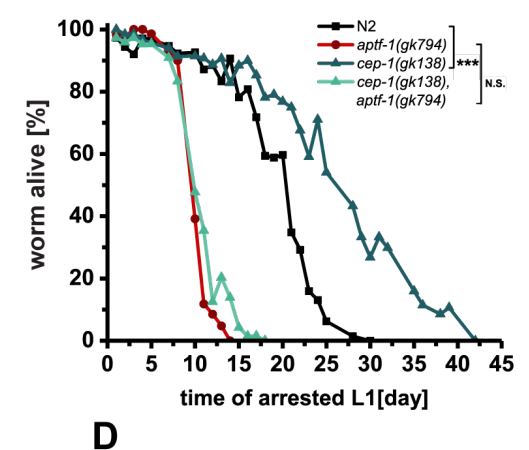

D

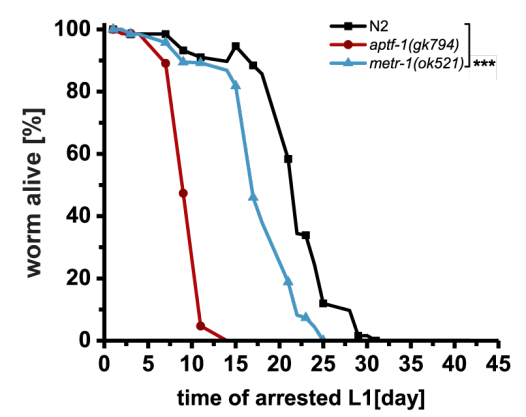

B

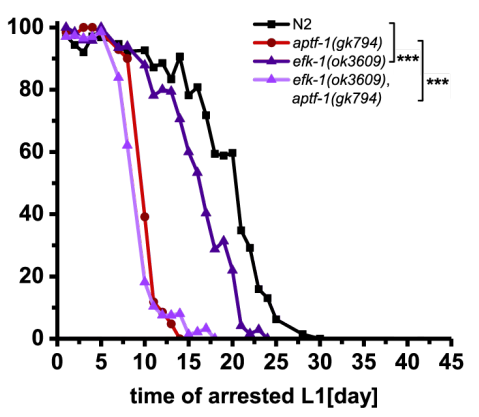

C

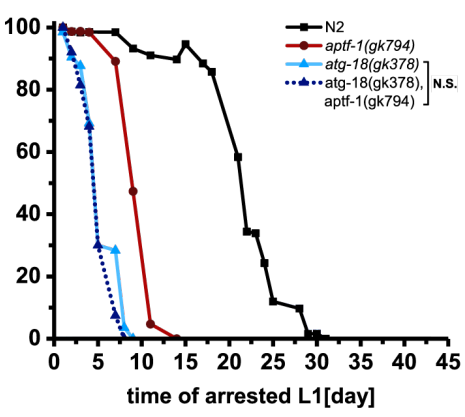

Figure 7 The sleep mechanism of sleep is related to apoptosis and autophagy.

(A) Lack of the gene cep-1 increased survival in arrested L1 larvae without food. No significant difference in survival spans was shown between arrested L1 double mutant cep-1(gk138)/aptf-1(gk794) and aptf1(gk794) mutants. (B) Arrested L1 efk-1(ok3609)/aptf-1(gk794) double mutants lived for slightly longer than aptf-1(gk794), ***p<0.001. (C) Arrested L1 atg-18(gk378)/aptf-1(gk794) double mutants and atg$18(\mathrm{gk378})$ lived for a much shorter length of time than aptf-1(gk794) mutant larvae. No significant difference was found between the two mutants. (D) Arrested L1 metr-1(ok521) mutants lived for a shorter length of time than wild-type worms but for longer than aptf-1 (gk794). Wild-type (N2) is shown in a black line and aptf-1(gk794) is shown in a red line. Mutants are shown in each figure, with different colors and symbols. To compare the $50 \%$ survival point of two lifespan groups, a Fischer's exact test was performed. Details of all the survival can be found in table S5.

\subsection{Mitochondrial respiratory chain is involved in the protective program of sleep}

Since sleep has been linked to the nutrient deprivation and energy balance in starved L1 C. elegans, I wondered whether impaired efficiency on the mitochondrial respiratory chain (MRC) is able to alter the survival of sleepless mutants. The deficiencies of the different complexes of MRC, which affect aging and alter lifespans in adult C. elegans have been investigated before. I thus crossed the MRC-complex mutants with sleepless mutant aptf-1 and tested the survival span of starved L1 larvae. 
Gene nио-6 (NADH ubiquinone oxidoreductase) encodes a subunit of complex I of MRC. The nuo-6 mutants show a functional reduction of complex I and an increase of lifespan of adult $C$. elegans [152]. Gene mev-1 (methyl viologen sensitive) encodes a subunit in complex II of MRC. It has been reported that mev- 1 mutants have a reduced lifespan with a high oxidative damage in the adult C. elegans [153-155]. The clk-1 (clock) gene is required for coenzyme Q $(\mathrm{CoQ})$ biosynthesis. CoQ locates in both innerand plasma membranes of mitochondria and transports electrons from the complex I and II to the complex III of MRC $[156,157]$. Lack of $c l k-1$ leads to $50 \%$ life extension in adult C. elegans [158]. The last gene is isp-1 (iron-sulfur protein). It serves in MRC complex III and encodes the rieske iron-sulfur protein. The isp-l mutant shows a respiratory reduction with decreased oxygen consumption and increased lifespan expectancy in adult $C$. elegans [159]. Interestingly, all the mutants above showed that a decreased fecundity has a prolonged development time in comparison with the wild-type C. elegans.

As shown in figure $8 \mathrm{~A}-8 \mathrm{D}$, no significant increase in survival was found in all the MRC mutants compared to wild-type L1 larvae during starvation, which suggests that MRC deficiency is not able to increase survival in starved L1 larvae. Interestingly, the double mutants isp-1/aptf-1, nuo-6/aptf-1, and mev-1/aptf-1 survived longer than the aptf-1 mutants (Figure 8A-8C). Deficiency of the MRC complex I, II and III was able to increase survival in the sleepless aptf-1 mutant in started L1 larvae. However, the ckl-1 mutants and the clk-1/aptf-1 double mutants showed a substantial decrease in survival, and the clk-1/aptf-1 double mutants lived for significantly less time than clk-1 mutants during L1-starvation (Figure 8D).

Moreover, the sleep of isp-1 L1 mutants decreased significantly during starvation when compared to the wild-type larvae. Also, the isp-1/aptf-1 double mutants lost almost all their sleep in L1 starvation (Figure S2A). Overall, these results indicate that MRC is involved in the protective program of sleep in starved L1 larvae. MRC complex I, II and III play essential roles in the downstream of sleep pathway while the CoQ act in a parallel pathway to increase survival in starvation. Besides, the reduction of sleep of the isp-1 mutant suggests that MRC might not merely act in the downstream pathway for helping survival but also affect the promotion of sleep. 
Figure 8

A

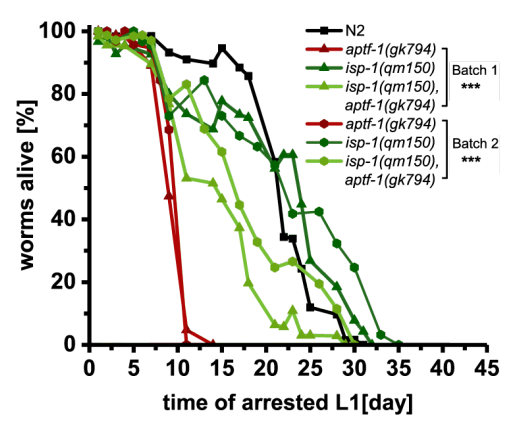

D

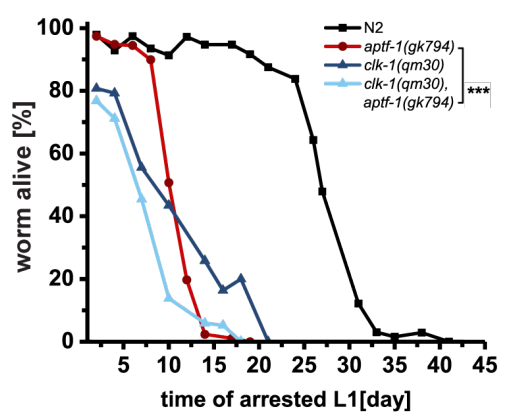

B

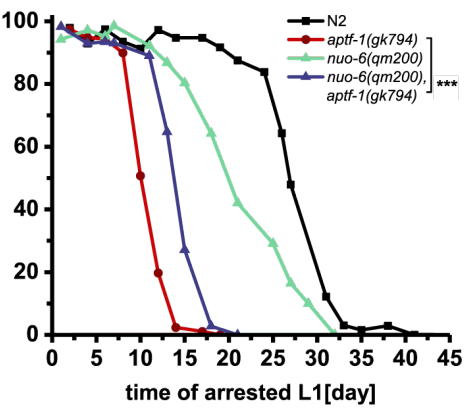

C

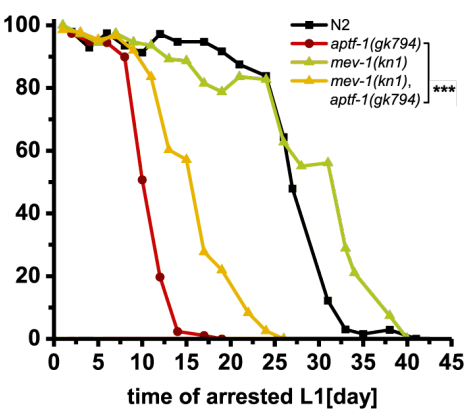

Figure 8 Sleep regulate survival through mitochondrial respiratory chain in arrested L1 larvae during starvation.

(A) Arrested L1 double mutant isp-1(qm150)/aptf-1(gk794) showed significantly longer lifespans than aptf-1 (gk794) in two replicates, $* * * p<0.001$. (B) Arrested L1 nuo-6(qm200) mutant larvae showed a reduced survival compared to the wild-type larvae The double mutant nuo-6(qm200)/aptf-1(gk794) lived for longer than the aptf-1(gk794) mutants, $* * * \mathrm{p}<0.001$. (C) The arrested L1 double mutant mev1(kn1)/aptf-1(gk794) showed an increased survival when compared to the aptf-1(gk794) mutants, $* * * \mathrm{p}<0.001$. (D) Both clk-1(qm30) mutants and clk-1(qm30)/aptf-1(gk794) double mutants lived for a much shorter length of time than wild-type L1 larvae during starvation. Double mutant clk-1(qm30)/aptf1 (gk794) showed a significant decrease in survival to the aptf-1(gk794) mutants. $* * * \mathrm{p}<0.001$. Wild-type (N2) is shown in a black line and aptf-1(gk794) is shown in a red line. Mutants are shown in each figure, with different colors and symbols. To compare the 50\% survival point of two lifespan groups, a Fischer's exact test was performed. Details of all the survival spans can be found in table S5.

\subsection{Sleep might contain two independent mechanisms for adult worms}

\section{and L1 larvae}

Lastly, I considered whether an increase in sleep could lead to raising the survival of starved L1 larvae, and thus I investigated the survival span in the sleepiness egl-4 mutant. The cGMP-dependent protein kinase (PKG) gain-of-function mutant egl-4 (ad450sd) has been shown to have an increased sleep time in the adult stage, as well as in lethargus [10]. The egl-4 (ks61) is a loss-of-function mutant [160]. However, the increase in survival was detected in neither gain-of-function nor loss-of-function L1 
mutants during starvation (Figure 9). Instead, both the egl-4 (ad450sd) and egl-4 (ad450sd) L1 larvae showed a significant decrease in survival during starvation. It indicated that the underlying mechanisms of lifespan extension by inducing sleep in the adult worms are distinct from the protective program of sleep during L1 starvation in $C$. elegans.

Figure 9

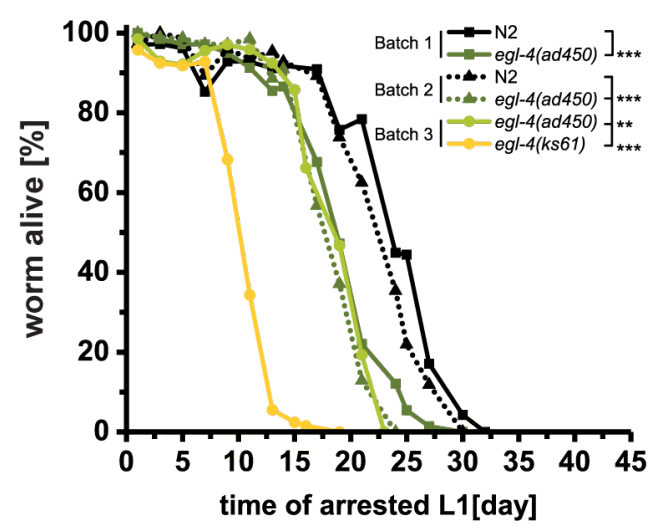

Figure 9 Increase of sleep adult worms did not influence the survival of arrested L1 larvae.

Replicates of the arrested L1 larva survival span of egl-4(ad450) and egl-4(ks61) mutants. Both mutants lived for a shorter length of time than wild-type larvae during starvation. Wildtype (N2) is shown in a black line. To compare the $50 \%$ survival point of two lifespan groups, a Fischer's exact test was performed. Details of the survival spans can be found in table S5.

\subsection{Sleep deprivation reduces the ROS generation in starved L1 larvae}

Reactive oxygen species (ROS) are a by-product of aerobic oxygen metabolism in mitochondria. It was believed that high-level ROS are able to damage macromolecules such as DNA and proteins, and low levels of ROS represent a low metabolic rate, linked to lifespan extension $[161,162]$. However, a shortened lifespan has been presented in worms which have low respiratory rates [163]. An increase in ROS can also be found in the worms, which have longevity caused by dietary restriction, down-regulating the insulin signaling or changing the mitochondrial metabolism in C. elegans $[164,165]$.

The primary product of ROS is the superoxide radical $\left(\mathrm{O}_{2}{ }^{-}\right)$, which is produced by the reduction of $\mathrm{O}_{2}$ and can be further dismutated to $\mathrm{H}_{2} \mathrm{O}_{2} \cdot \mathrm{H}_{2} \mathrm{O}_{2}$ is the precursor of different cytotoxic compounds, known as ROS. It has been reported that in C. elegans, the $\mathrm{H}_{2} \mathrm{O}_{2}$ level is very high in the larval development, but declines in the period of reproduction, then rising again with age in adult worms [166]. Importantly, it has been observed that the arrested L1 larvae are also able to accumulate ROS over survival time, like adult worms [117]. Therefore, ROS might play a very interesting role in starvation survival in 
L1 larvae. To test this hypothesis, I used ROS-sensitive dye dihydroethidium (DHE) to quantify ROS in L1 larvae of wild-type worms, aptf-1 mutants, and RIS ablated worms during starvation. DHE reacts with ROS to form a red fluorescent product 2hydroxyethidium in chromatin. This fluorescent can be visualised and quantified easily by using a microscope [167].

The representative images of ROS in starved L1 larvae are shown in figure 10A. I quantified the ROS mutant in the head region, in order to avoid interference of autofluorescence from the worm's body (Figure 10B). ROS accumulated and increased in all three strains over the survival span in starved L1 larvae. Unexpectedly, ROS rose more slowly in the sleep-deprived L1 larvae, aptf-1 mutants and RIS ablated worms than in wild-type larvae during starvation. Starting on the eighth day, the ROS levels in sleepdeprived worms are significantly lower than those in wild-type worms. It suggested that sleep might able to increase the ROS generation in chromatin for promoting the survival of starved L1 larvae. 
Figure 10

A

ROS-indicator DHE signal in the head of arrested L1 larvae

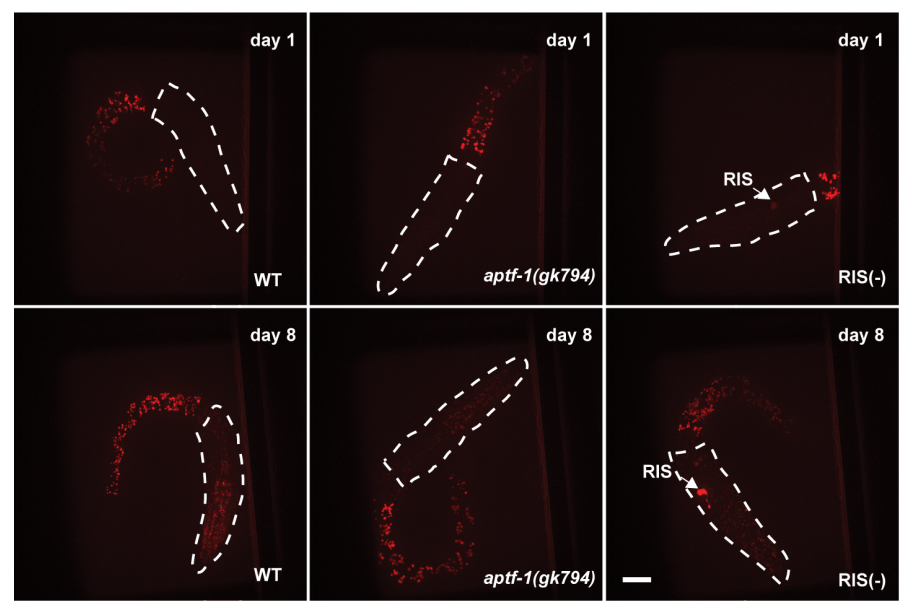

B

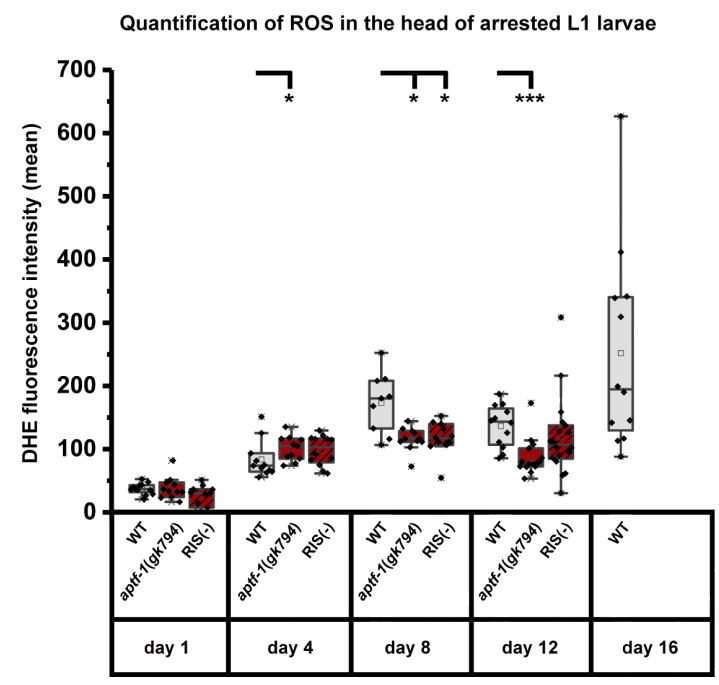

Figure 10 Sleep influences the reactive oxygen species (ROS) production in the head of arrested L1 larvae during aging.

(A) Images of ROS generation in the head regions of 1-day old and 8day old arrested L1 larvae. N2, wild-type worm; aptf-1(-), mutant worm aptf-1(gk794); RIS (-), RIS ablated worm. ROS level was visualised by the indicator DHE staining using a microscopy. Scale bar, $10 \mu \mathrm{m}$. (B) Quantification of ROS level in the head of arrested L1 during aging. Aged sleepless mutants generated less ROS in head than wild-type arrested L1 larvae during starvation. Individual worm was shown in the box plot as dots, $\mathrm{n}>10$ for each day of each strain. 4days: $\mathrm{N} 2: 83.6 \pm 8.8, \mathrm{n}=11$; aptf1(gk794): $101 \pm 5.9, \mathrm{n}=11, * \mathrm{p}<$ 0.05 ; RIS (-): $98 \pm 6.8, \mathrm{n}=13$. 8day: $\mathrm{N} 2: 173.1 \pm 15.9, \mathrm{n}=9$; aptfl(gk794): $115.9 \pm 5.6, \mathrm{n}=11, * \mathrm{p}<$ 0.05 ; RIS (-): $117.4 \pm 7.9, \mathrm{n}=11, * \mathrm{p}$ $<0.05$. 12-days: $\mathrm{N} 2: 136.5 \pm 9.6, \mathrm{n}$ $=12 ; \operatorname{aptf}-1(g k 794): 86.9 \pm 7.4, \mathrm{n}=$ $15, * * * \mathrm{p}<0.001 ;$ RIS (-): $119.4 \pm$ $14.8, \mathrm{n}=18$. Mann-Whitney U test.

\subsection{Sleep increases the redox stage throughout of survival in arrested}

\section{L1 larvae.}

A complex cellular homeostasis (proteostasis) network organizes and coordinates several biological processes in protein synthesis, folding, maintenance and clearance. The network plays a role in protein quality control and provides protection against protein misfolding and aggregation [168]. It associates with redox homeostasis and ROS accumulation, and serves as an important factor in age-related diseases [169]. In $C$. elegans, protein homeostasis decline during aging and the main processes are focused on the cytosolic compartment $[170,171]$. 
In order to know if the redox homeostasis in the cytosol can be affected by aging and sleep in arrested L1s, I measured the redox state in vivo using a genetically encoded sensor: redox-sensitive green fluorescent protein (roGFP). RoGFP is an engineered GFP. It contains two cysteine residues with the dual excitation at $405 \mathrm{~nm}$ or $488 \mathrm{~nm}$ respectively, and a single emission at $509 \mathrm{~nm}$ [172]. In vivo, the oxidizing condition increases the formation of the disulfide bond and therefore leads to a higher $405 \mathrm{~nm}$ excitation and a lower $488 \mathrm{~nm}$ excitation. Shifting to the reducing condition lowers the disulphide bond and results in a reduced excitation at $405 \mathrm{~nm}$ and higher one at $488 \mathrm{~nm}$. The roGFP allows for measuring the signal at both $405 \mathrm{~nm}$ and $488 \mathrm{~nm}$ at the same time, and therefore serves as an ideal tool for detecting the redox state in vivo.

To obtain the cytosolic redox state in C.elegans, I used two strains: the F53B3.3roGFP and the unc54roGFP, which represent the redox homeostasis specifically in the panneuronal and muscular cytosols. The measurement was performed throughout the lifespan of the arrested L1s. 6 to 15 worms of different arrested times were imaged by microscope. A clear increase of a 405/488 nm ratio along with aging is shown in both neuronal cytosol (Figure 11A) and muscular cytosol (Figure 11B). It indicates that the redox state in pan-neuronal and muscular cytosols becomes more oxidized during aging in arrested L1s in both wild-type and aptf-1 mutants. This result is similar to the observation made in a previous study, which reported that the cytosolic redox state became more oxidized during development and aging in adult worms [132].

Furthermore, the cytosol in sleepless mutants is generally more reducing than that of wild-type worms of the same age. The $405 / 488 \mathrm{~nm}$ ratio of aptf- 1 mutants in the middleaged worms ( 8 days) is significant lower in both pan-neuronal $(-35.3 \%, * * * p<0.001)$ and muscular cytosol $(-46.2 \%, * * * p<0.001)$ than the ratio of the wild-type worms. This result is similar to the measurement of ROS levels in the experiment above, which shows a decreased ROS in aptf-1 mutants when compared to wild-type worms. 
Figure 11

A Ratio of roGFP in neuronal cytosol of arrested L1 larvae

B Ratio of roGFP in muscular cytosol of arrested L1 larvae
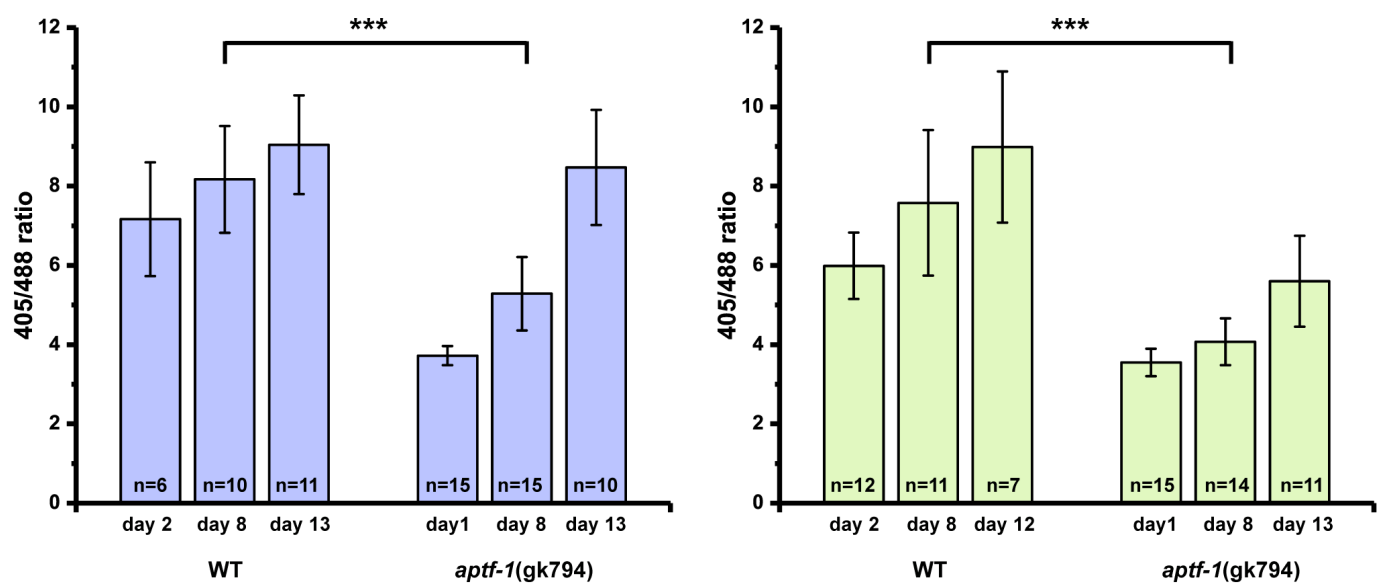

Figure 11 The 405/488 roGFP ratio in the sleepless mutants was lower than the ratio in wild-type L1 larvae during starvation.

(A) The ratio of pan-neuronal roGFP of arrested L1 larvae. F53B3.3roGFP was expressed in pan-neurons and represented the redox level. Redox of the early-arrested L1 larvae $\left(1^{\text {st }}-2^{\text {nd }}\right.$ day), middle-arrested L1 larvae $\left(8^{\text {th }}\right.$ day) and the late-arrested L1 larvae $\left(12^{\text {th }}-13^{\text {th }}\right.$ day) were shown. $8^{\text {th }}$ day: wild-type $(\mathrm{N} 2): 8.2 \pm$ 0.4; aptf-1(gk794): $5.3 \pm 0.2$. ***P $<0.001$. (B) The ratio of roGFP in muscular cytosol of arrested L1 larvae during starvation. unc54roGFP was expressed in muscle cells and represented the redox levels there. $8^{\text {th }}$ day: wild-type (N2): $7.6 \pm 0.5$; aptf-1 $(g k 794): 4.0 \pm 0.1 . * * * \mathrm{P}<0.001$, Mann-Whitney U test.

\subsection{Sleep did not affect the cell-cycle quiescence in L1 larvae during starvation}

It has been shown in previous studies that in the arrested L1 C. elegans, the germline stem cells Z2 and Z3 have undergone cell-cycle quiescence and were arrested at the G2 phase during starvation. A tumor suppressor DAF-18/PTEN was identified to be indispensable in this cell-cycle quiescence. The germ cells Z2 and Z3 in the daf-18 arrested L1 mutant were unable to maintain the cell quiescence and continually grew and divided into 5 to 7 germ cells $[42,173]$. Since I found that the daf-18 mutant lacks sleep during L1 starvation and DAF-18/PTEN is involved in the sleep-active/sleep-promoting pathway and protective program of sleep during starvation [118] (see Figure 3-C), I questioned whether the aptf-1 arrested L1 mutant might not able to keep the same germline cell-cycle quiescence as the daf-18 L1 mutant during starvation. I thus visualized the $\mathrm{Z} 2$ and $\mathrm{Z} 3$ cells using ppie-1::GFP in starved aptf-1 L1 larvae. The representative images of $\mathrm{Z} 2$ and $\mathrm{Z3}$ cells in starved L1 larvae are shown in figure 12A. 
Just as in the wild-type control larvae, aptf-1 L1 larvae showed only two germ cells, regardless of how long the worm was starved for. I further detected the number of germ cells in larvae after feeding them with bacteria for 11 hours and 46 hours respectively (Figure 12B). As shown in figure 12C, no significant difference in the division of germ cells was found in aptf-1 larvae when compared to wild-type larvae after feeding for 11 hours. The negative results revealed that the cell-cycle arrest of germ cell is independent of sleep in L1 larvae during starvation.

Figure 12

A

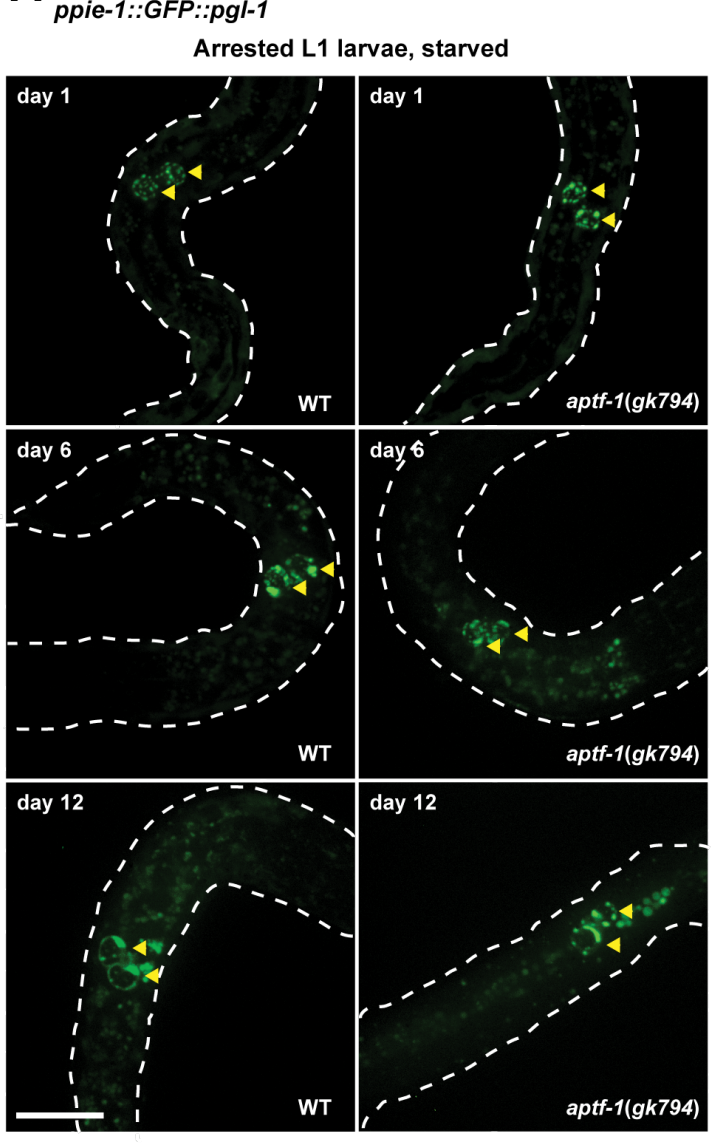

B ppie-1::GFP::pgl-1

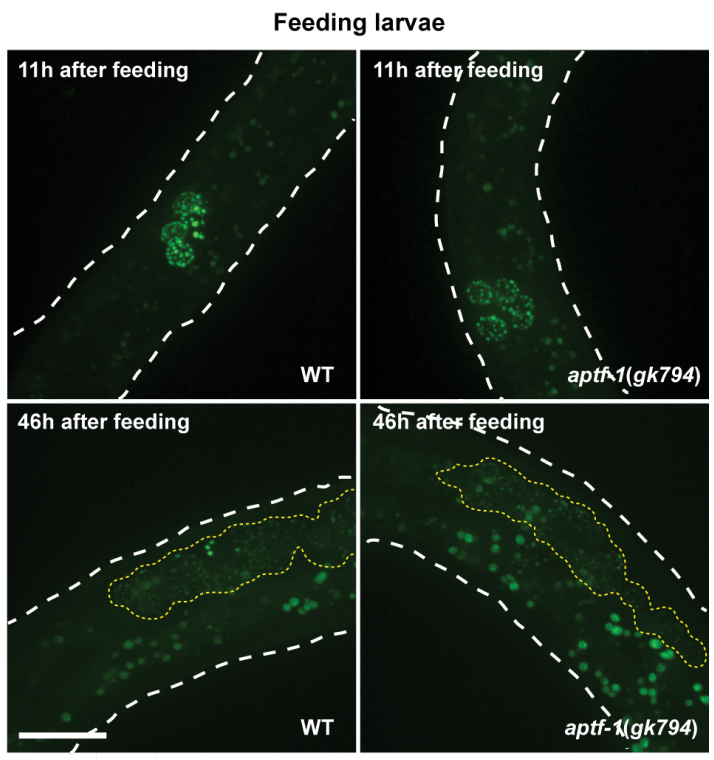

C

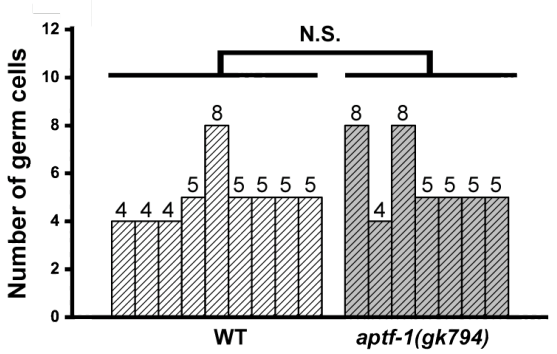

Figure 12 Arresting germ cell division is not influenced by sleep in arrested L1 larvae during starvation.

(A) The growth of germ cells (Z2 and Z3) was arrested in L1 diapause. ppie-1::GFP was expressed in Z2 and $Z 3$ cells in the wild-type and the aptf- 1 sleepless mutants ( 1 day, 6 days, 12 days-arrested L1 larvae) are shown in the images. The yellow triangular symbols are shown for the location of the two germ cells. Scale bar, $10 \mu \mathrm{m}$. (B) Images of germ cell division in worms after feeding. The arrested L1 larvae were fed for 11 hours and 46 hours. Yellow dotted lines show the region of germ cells. Scale bar, $10 \mu \mathrm{m}$. (C) Number of divided germ cells in L1 after feeding for 11 hours. One bar represents the number of germ cells in a single worm. There was no significant difference between wild-type worms and the aptf-1 mutant worms. Wild-type (N2): $5 \pm 0.4, \mathrm{n}=9$; aptf-1 (gk794): $5.7 \pm 0.6, \mathrm{n}=7$, N.S. Mann-Whitney U test. 


\subsection{Sleep improves the unfolded protein response in resisting ER stress in starved L1 larvae}

The endoplasmic reticulum (ER) is responsible for protein translation, folding, and transportation. It balances the intracellular calcium stage and serves an irreplaceable role in cell death [174]. Aging and starvation disturb protein folding and lead to the accumulation of unfolded and misfolded proteins in the ER and thus induce ER stress $[175,176]$. The heat shock protein HSP-4 is a homolog of grp78/BiP (glucose-regulated protein 78/immunoglobulin heavy chain-binding protein) of mammals. It plays an impotent role in UPR and shows an up-regulation in response to ER stress [177]. HSP-4 regulates the viability of embryos and larvae. It can be found in hypodermis, intestines, and spermatheca in C. elegans.

To investigate whether sleep is able to affect the UPR in ER stress during larval starvation, I tested the $h s p-4$ expression in starved L1 larvae and observed a significant reduction of $h s p-4$ in the long-starved aptf-1 larvae when compared to controlled larvae. As shown in the representative images in figure 13A, phsp-4::GFP expressed in the hypodermis, intestines of the starved L1 larvae. The quantification of the phsp-4::GFP intensity showed that $h s p-4$ expression increased along with the survival time in the wild-type larvae but not in the aptf-1 larvae during starvation (Figure 13B). In contrast, the long-starved aptf-1 larvae expressed less HSP-4 than the short-starved aptf-1 larvae. This suggests that sleep might play a role in UPR against starvation and avoid cell damage in starved L1 larvae. 
Figure 13
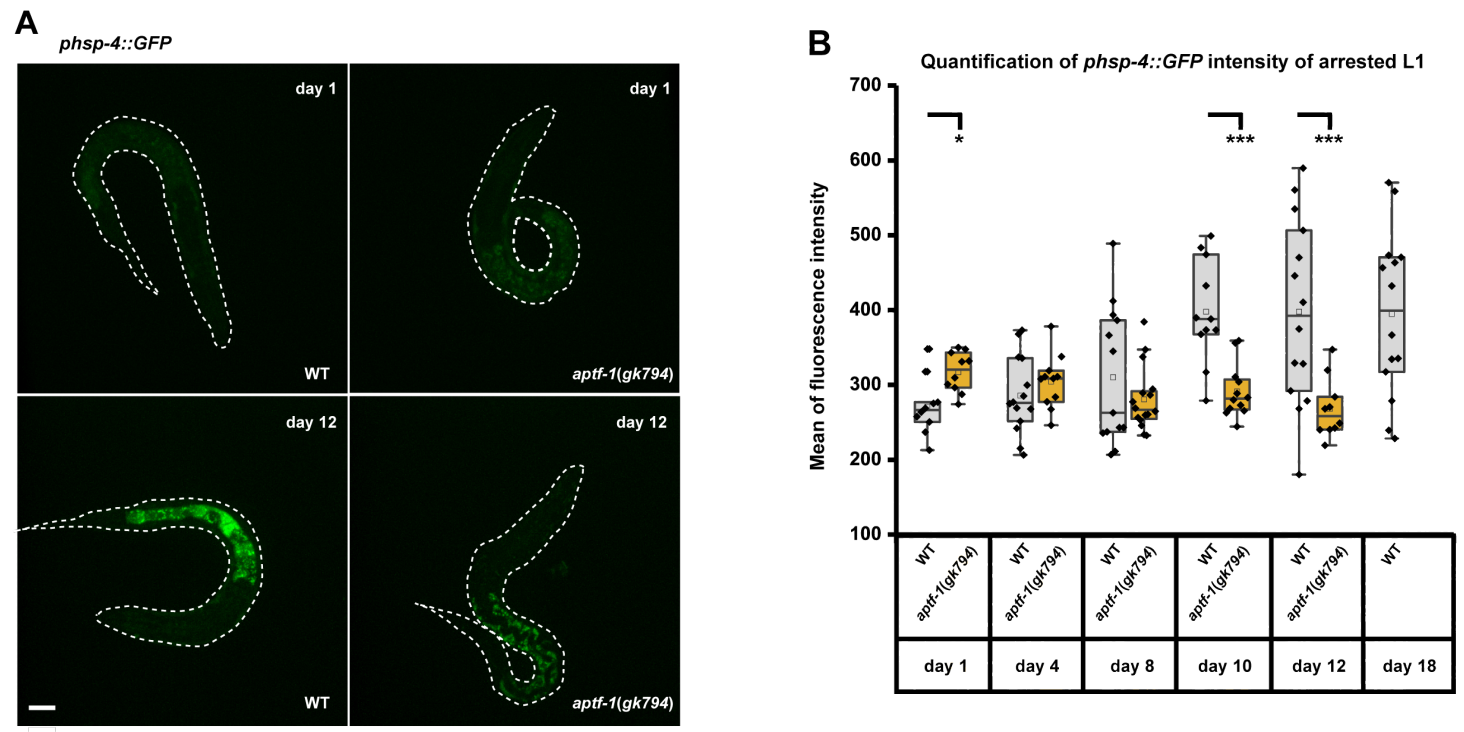

Figure 13 Sleep increases the HSP-4 expression during aging.

(A) phsp-4::GFP expression in arrested L1 larvae during aging. 1 day old and 12 days old arrested L1 larvae were shown. N2, wild-type worm; aptf-1(-), mutant worm aptf-1(gk794). Scale bar, $10 \mu \mathrm{m}$. (B) Quantification of the HSP-4 expression of the whole worm in fluorescence intensity. Individual worm's mean intensity of phsp-4::GFP is shown in a single dot. $\mathrm{n}>10$ of each strain on each experiment day. 1day: $\mathrm{N} 2: 270.7 \pm 12.2, \mathrm{n}=10 ;$ aptf-1 $(g k 794): 317.1 \pm 8.5, \mathrm{n}=10, * \mathrm{P}<0.05 .10$-day: $\mathrm{N} 2: 397.9 \pm 20.8, \mathrm{n}$ $=11 ; \operatorname{aptf}-1(g k 794): 291.2 \pm 10.3, \mathrm{n}=12, * * * \mathrm{P}<0.001$. 12-day: $\mathrm{N} 2: 397.7 \pm 33.3, \mathrm{p}=14$; aptf-1(gk794): $267.9 \pm 12.5, \mathrm{n}=10, * * * \mathrm{P}<0.001$. Mann-Whitney $\mathrm{U}$ test.

\subsection{Sleep promotes the $h s p-16.2$ expression after heat shock in early- starved L1 larvae}

The heat shock protein HSP-16.2 is responsible for unfolded protein binding activity and can be induced by heat shock. In C. elegans, HSP-16.2 is involved in lifespan extension of adult worms, and plays a resistant role in proteotoxic stress [178-180].

To further understand the protective role of sleep in UPR mechanisms against stress in starved L1 larvae, I investigated the expression level of hsp-16.2 in sleepless mutant aptf-1 after heat shock. Here, increasing the heat-shock temperature can subsequently increase the expression level of $h s p-16.2$ during starvation. However, regardless of which temperature was used, the 1-day-arrested aptf-1 L1 larvae expressed significantly lower $h s p$-16.2 than the 1-day arrested wild-type L1 larvae during starvation (Figure 14A-14C). In the 9-day arrested larvae, no difference in $h s p-16.2$ expression was found between the aptf-1 mutants and wild-type worms (figures 14D, 14E). 
Interestingly, the 9-day arrested L1 larvae, which had been already heated in the first arrested day at $37^{\circ} \mathrm{C}$ for 1 hour, only showed a slight increase in $h s p-16.2$ expression after re-heating at $37^{\circ} \mathrm{C}$ for 1 hour. Also, the expression of $h s p-16.2$ in aptf- 1 larvae was lower than the wild-type larvae after re-heat shock (Figure 14F).

Figure 14G shows the difference in $h s p-16.2$ expression after heat shock between aptf-1 and wild-type of 1-day arrested larvae. The mean intensity of phsp-16.2::GFP in the L1 larvae are quantified and displayed in figure $14 \mathrm{H}$. Taken together, the results indicate that sleep increases $h s p-16.2$ expression in short-starved L1 larvae and seems to play an elevated role in a protective program against heat shock stress. Moreover, this protective mechanism could be remembered after heat-shock by unknown factors and thus further influence the C. elegans larvae over time. Notwithstanding, further evidence, such as the survival rate of aptf-1 mutants after heat shock, is required to fully understand the implication of sleep in response to heat shock stress. 
Figure 14
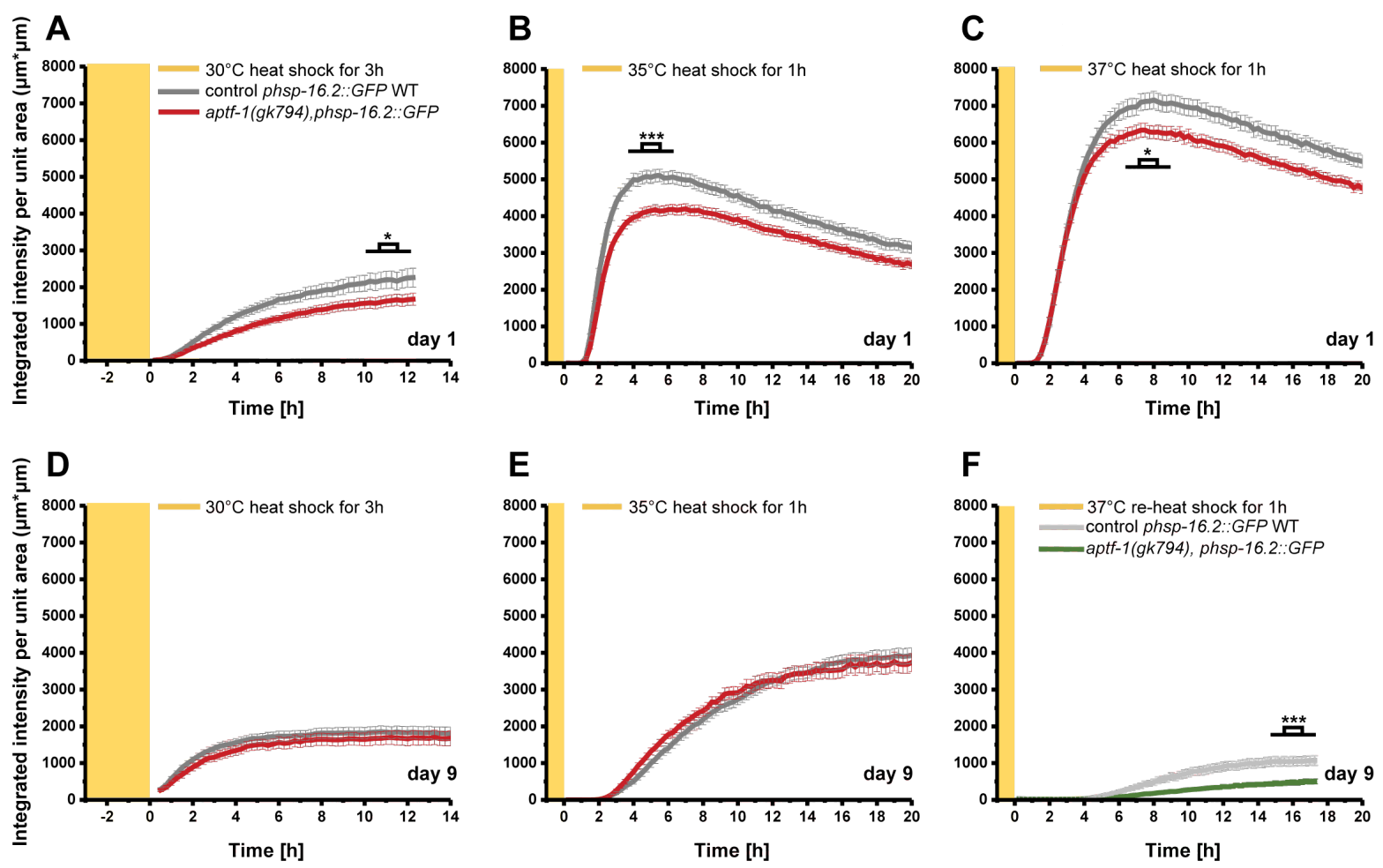

G

H
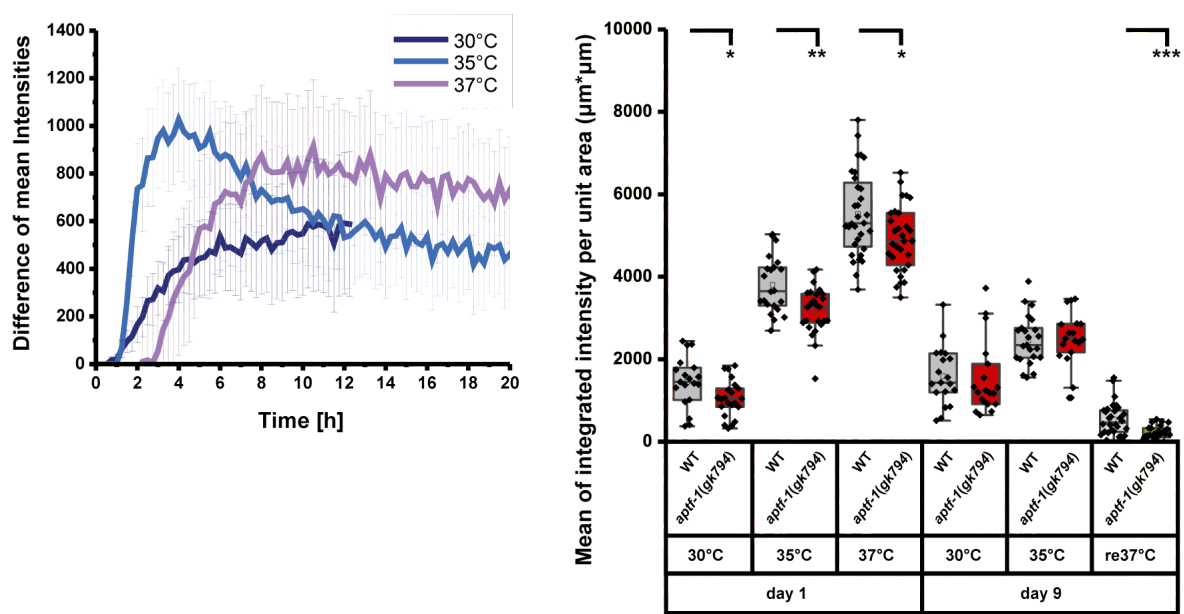

Figure 14 Expression of phsp-16.2::GFP was increased by sleep after heat shock in young arrested L1 larvae.

Expression of phsp-16.2::GFP in 1-day arrested L1 larvae increased after (A) $30^{\circ} \mathrm{C}$ heat shock for 3 hours, wild-type: $\mathrm{n}=19$, aptf-1 $(g k 794): \mathrm{n}=22$; (B) $35^{\circ} \mathrm{C}$ heat shock for 1 hour, wild-type: $\mathrm{n}=22$, aptf1 (gk794): $\mathrm{n}=25$; (C) $37^{\circ} \mathrm{C}$ heat shock for 1 hour wild-type: $\mathrm{n}=32$, aptf-1 $(\mathrm{gk794}): \mathrm{n}=30$. The increase in the temperature of the heat shock caused the rise of the phsp-16.2::GFP expression level in arrested L1 larvae. Expression of phsp-16.2::GFP in 9-day arrested L1 larvae after (D) $30^{\circ} \mathrm{C}$ heat shock for 3 hours, wild-type: $\mathrm{n}=19$, aptf-1 $(\mathrm{gk} 794): \mathrm{n}=19$; (E) $35^{\circ} \mathrm{C}$ heat shock for 1 hour wild-type: $\mathrm{n}=23$, aptf-1 (gk794): $\mathrm{n}=18$. The expression in the 9-day arrested L1 larvae was not as high as the expression in the 1-day arrested L1 larvae. (F) For the re-heat shock treatment, L1 larvae were heat-shocked with $37^{\circ} \mathrm{C}$ for 1 hour on the first arrested day and maintained in the chamber at $20^{\circ} \mathrm{C}$ for 8 days, continually. On the 9 -day, the worms were re-heat shocked again with $37^{\circ} \mathrm{C}$ for 1 hour. The expression level was shown in the figure, wild-type: $\mathrm{n}=30$, aptf-1 $(\mathrm{gk} 794): \mathrm{n}=26$. (G) Deference of $p h s p-16.2: \because G F P$ expression in wild-type larvae and aptf-1 mutant larvae after heat shock. (H) Mean of phsp-16.2::GFP expression in deferent arrested L1 larvae after heat shock with $30^{\circ} \mathrm{C}$ for 3 hours, as well as $35^{\circ} \mathrm{C}$ and $37^{\circ} \mathrm{C}$ for 1 hour. 1-day arrested L1 
mutants aptf-1 $(g k 794)$ showed significantly lower expressions than the wild-type larvae after heat shocks $\left({ }^{*} \mathrm{P}<0.05\right.$ of $30^{\circ} \mathrm{C}$; ${ }^{*} \mathrm{P}<0.01$ of $35^{\circ} \mathrm{C}$; ${ }^{*} \mathrm{P}<0.05$ of $37^{\circ} \mathrm{C}$, Mann-Whitney U test. In the 9-day arrested L1 larvae, this difference was no longer displayed. The re-heat shocked 9-day arrested mutant L1 larvae showed a significant reduction, $* * * \mathrm{P}<0.001$, Mann-Whitney $\mathrm{U}$ test.

\subsection{Energy exhaustion is not the main reason for the premature death of starved L1 larvae}

Since the survival of arrested L1 larvae is linked to the length of starvation, I wondered whether sleep is able to decrease energy expenditure and therefore increase the survival time of larvae without the energy intake as long as possible. Because the storage and utilisation of fat is a major factor in energy balance, measuring fat status might help us to infer the energy expenditure in starved L1 larvae over survival span. To test this idea, I stained the fat granules in L1 larvae using the fatty C1-BODIPY 500/510 C12 acid [129].

Consistent with a previous study by Srinivasan, in which food deprivation depletes the fat stores in C. elegans [181], I found that the fat mass decreased dramatically over the survival span in all three L1 larvae: wild-type worms, aptf-1 mutants, and RIS ablated worms during starvation. The representative images of fat granules of starved L1 larvae are shown in figure 15A. In the 7-day of starvation of the wild-type larvae, $90 \%$ of the total fat storage had been depleted (Figure 15B). This raises an intriguing question: if we calculate the total survival time (wild-type arrested L1 larvae can survive about 23 days during starvation) and the total energy consumption as a percentage, how could the starved larvae use $10 \%$ of the total energy to survive $70 \%$ of the time of the L1 arrest? It indicated that the energy shortage is not the only factor determining survival. There must be other mechanisms or factors that regulate survival in starvation together with energy homeostasis in C. elegans.

Interestingly, the sleep-deprived aptf- 1 larvae and RIS ablated larvae contained more fat granules than the wild-type larvae, especially at the early arrest time. It suggests that sleep might also relate to fat utilisation in L1 larvae during starvation. 


\section{Figure 15}

\section{A}

C1-BODIPY-C12 lipid staining in N2, aptf-1(gk794) and RIS(-) arrested L1 larvae
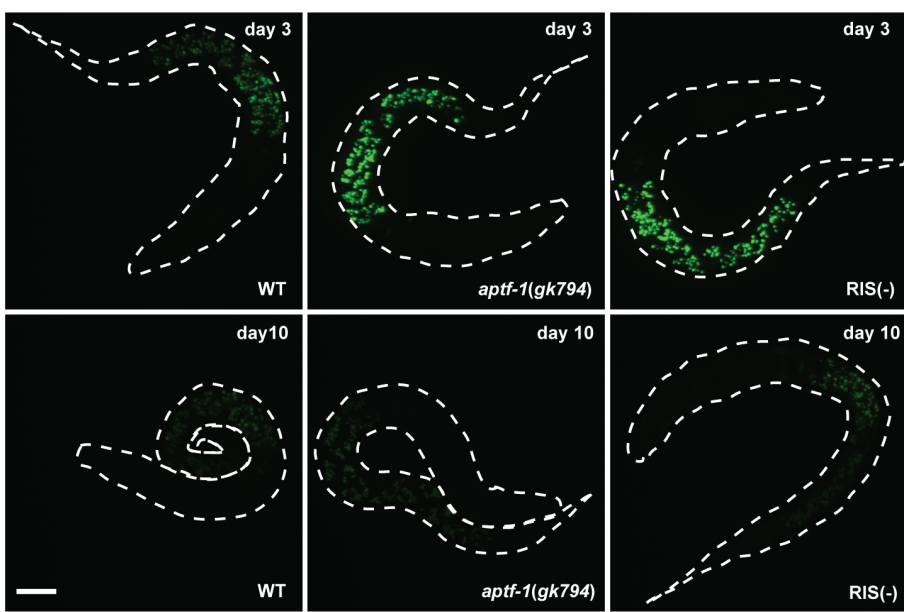

B

Abundance of fat granules in arrested L1 larvae

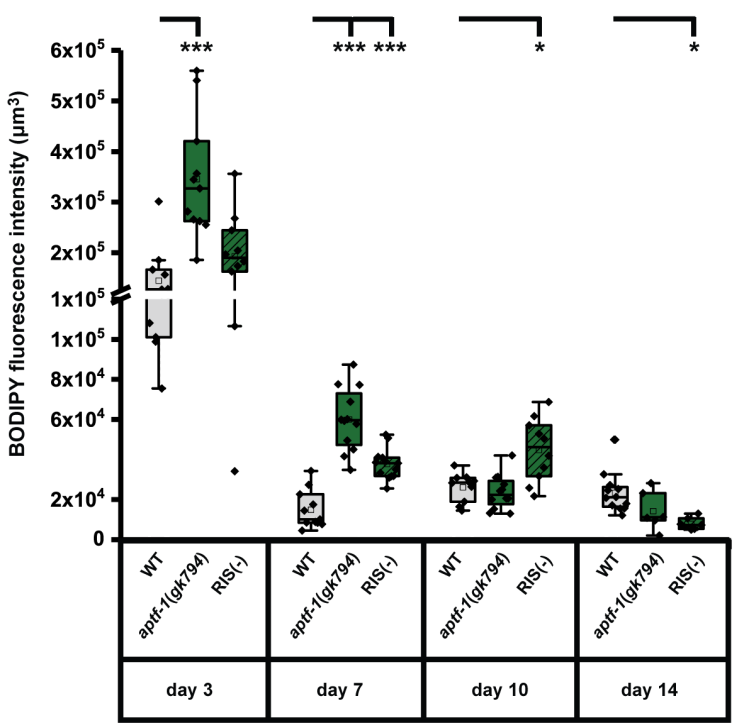

Figure 15 Sleepless mutants contain more fat content than wild-type arrested L1 larvae in the early time of starvation.

(A) Images of fat granules in body of arrested L1 larvae during starvation. The fat granules were stained with the bodipy-C12 fatty acid for $48 \mathrm{~h}$ and visualised using microscopy. Images of 3-day and 10-day arrested L1 larvae were shown. N2, wild-type worm (WT); aptf-1(-), mutant worm aptf1(gk794); RIS(-), RIS ablated worm. Scale bar, $10 \mu \mathrm{m}$. (B) Quantification of the fluorescence intensity of fat granules in the whole worm of arrested L1 larvae. The mean fluorescence intensity of a single worm was shown in a single dot, $\mathrm{n}>10$ for each day of each strain. 3-day: N2: $1.4^{*} 10^{5} \pm 2 * 10^{4}, \mathrm{n}=10$; aptfl(gk794): $3.5^{*} 10^{5} \pm 3.6^{*} 10^{4}, \mathrm{n}=11$, $* * * \mathrm{p}<0.001 ; \quad$ RIS $\quad(-)$ : $1.9 * 10^{5} \pm 2.8 * 10^{4}, \mathrm{n}=10.7$-day: $\mathrm{N} 2$ : $1.5^{*} 10^{4} \pm 2.9^{*} 10^{3}, \mathrm{n}=11$; aptfl(gk794): $6 * 10^{4} \pm 4.5^{*} 10^{3}, \mathrm{n}=12$, $* * * \mathrm{p}<0.001 ;$ RIS (-): $3.8 * 10^{4} \pm$ $2.1 * 10^{3}, \mathrm{n}=13, * * * \mathrm{p}<0.001$. 10day: N2: $2.6^{*} 10^{4} \pm 2.3 * 10^{3}, \mathrm{n}=10$; aptf-1 (gk794): $2.4 * 10^{4} \pm 2.5 * 10^{3}, \mathrm{n}=$ 12; RIS (-): $4.5^{*} 10^{4} \pm 5^{*} 10^{3}, \mathrm{n}=10$, ${ }^{*} \mathrm{p}<0.05$. 14-day: N2: $2.3 * 10^{4} \pm$ $2.9^{*} 10^{3}, \quad \mathrm{n}=12 ; \quad$ aptf-1 $(\mathrm{gk} 794)$ : $1.4^{*} 10^{4} \pm 3.9 * 10^{3}, \mathrm{n}=6$; RIS (-): $7.9^{*} 10^{3} \pm 1.1 * 10^{3}, \mathrm{n}=7,{ }^{*} \mathrm{p}<0.05$. Mann-Whitney U test. 


\section{Discussion}

\subsection{The role of sleep in adult $C$. elegans during starvation}

Since C. elegans became a powerful tool for sleep research in the past 15 years, three states of worm sleep have been found and well characterized in the literature. The developmentally-timed sleep, lethargus, occurs only in the larval development period before each of the four molts. Another stage, stress-induced sleep, can be activated in both adult- and larval worms by various stress stimulations. Feeding of starved worms with high-quality food is able to activate a sleep stage called satiety quiescence. So far, however, there have been no controlled studies which compare functional differences between the three sleep stages. The core function of sleep in $C$. elegans is still unknown.

In the previous study, Masurat and I found that starvation-induced sleep occurs not only in starved adults but also in arrested L1 larvae. Several quiescence bouts with a cyclic quiescence-active-quiescence pattern were detected in worms during starvation. As shown in the paper above, this starvation-induced sleep can be activated by AMPactivated kinase and FoxO, and shares the same sleep-active/sleep-promoting mechanism through the single neuron RIS in adult worms and arrested L1 larvae. Sleep is essential for survival of L1 larvae during starvation but dispensable for the survival of starved adult worms. This suggests that sleep might have distinct functions in adult worms and L1 larvae during starvation.

Sleep seems needless in adult worms in the presence of sufficient food, especially when the worms live under a comfortable laboratory growth condition. Interestingly, sleep exists in the starved worms for unknown reasons. According to Skora's study, the brain dynamics decrease in starved adults during sleep. She thus suggests that sleep might serve as an adaptive strategy of energy expenditure in neuronal signaling in combination with the reduction of muscle activity during starvation [182]. However, this adaptive strategy of sleep does not seem to benefit the survival of starved adult worms. Sleepless aptf-1 adult mutants live as long as the sleeping worms whether there is food or not [118]. It indicates that even though sleep is triggered by starvation, it is not involved in the mechanisms of this lifespan extension caused by starvation. 
I further tested the longevity mechanisms such as metabolic alterations, the IIS signaling pathway, and the TOR signaling pathway. None of those mechanisms are related to sleep for lifespan extension. Thus, besides energy conservation, sleep should have an additional function in adult worms during starvation. In other words, exhaustion of energy might not be a decisive factor leading to death in adult worm. On the other hand, resource allocation has been proposed to be another function of sleep [183, 184]. However, evidence from future studies is required to account for the primary function of sleep in adult worms during starvation.

\subsection{Mechanism of sleep in arrested L1 larvae during starvation}

I questioned why sleep is not required in adult worms for lifespan extension but seems indispensable in the arrested L1 larvae for maintaining survival during starvation. It could be hypothesized that the functions of sleep in arrested L1 larvae might be distinct from those in adult worms, even though they share the same sleep-active/sleeppromoting pathway through AMPK and FoxO in upstream of RIS activation pathway.

To understand why sleep functions differently in adults from larvae, we first have to underscore the major biological difference between adult worms and larval worms during starvation. Contrary to the larvae, adult worms in the absence of food develop fully and might contain complete anti-stress mechanisms. Therefore, the larvae may have to enter an emergency state called "arrest" to inhibit all the cell division and increase the resistance to environmental stress [122].

The L1 arrest is different from the dauer arrest. Dauer arrest is an alternative third larval stage with modified morphology. Dauer can be induced by environmental stresses such as overcrowding, scarcity of food and overheating within certain limits. The dauer larvae are able to survive for several months during starvation until food becomes available [185]. In contrast, arrested L1 larvae that caused by starvation after hatching can only live for a couple of weeks without food [40]. 


\subsection{Sleep and aging}

Roux showed that over the arrested time, L1 larvae have a similar cellular decline to normal aging process. He tested several biological aging markers such as ROS production, mitochondrial fragmentation, and protein aggregation. All of them increased over time in the arrested L1 larvae [117]. Moreover, the larvae also showed a decreased proteotoxic-stress resistance and a decline of proteostasis. Recent works of literature have been shown that the genes, which relate the longevity of adult worms, also affect the survival of arrested L1 larvae [40]. The associated mechanisms include the insulin/IGF-1 signaling, the heat-shock factors, the AMP kinase, and the sensory perception process as well as the translation process [186-189].

Because sleep is an essential regulator for survival in arrested L1 larvae, I wondered whether sleep could accelerate or retard the aging process and, sequentially, influence survival in L1 larvae. My study provided ample evidence that sleepless mutant aptf-1 larvae display an increased muscle disruption, mitochondrial fragmentation, and protein aggregation. It suggests that sleep is able to counteract the aging-induced phenotypes that occur in the starved larvae in L1 arrest.

Thus, in the absence of food, the sleep of L1 larvae functions not only as energyeconomizing medium against starvation but also as a protective strategy to counter aging. In this study, I firstly reporte an association between sleep and aging in arrested L1 larval C. elegans. The results provide insights for understanding why sleep changes with age in human beings. In the previous body of research, the poor onset and maintenance of sleep observed in old adults are considered to be due to the impairment of homeostatic sleep regulation, which is associated with normative aging [190-192]. The central brain mechanisms have been proposed to serve as regulators of the age-related sleep. However, other factors such as obesity, chronic pain, hormonal changes, and neurodegeneration as well as psychiatric conditions are all involved in sleep disruption in aged adults [193195].

Thus, aging might not be the determinate of sleep reduction in aged adults. It is not clear how the quality and quantity of sleep affect the aging process in human. If sleep acts in a 
protective strategy to slow down the aging process in L1 larval C. elegans, it should be involved in numerous fundamental biological mechanisms related to aging.

\subsection{Sleep, apoptosis, and autophagy}

Indeed, it was found that sleep was associated with apoptotic cell death and autophagy in arrested L1 larvae. Autophagy has an overlapped mechanism with apoptosis [196]. It is believed that autophagy is able to protect cells and extend survival during starvation [197]. Apoptotic machinery acts not only in the development process to maintain tissue homeostasis but also functions as a failsafe program to protect living organisms under adverse conditions while none of the others repair or protective mechanisms are effective. Therefore, autophagy, apoptotic and sleep might have the same purpose and function as one of the physiologic safeguards to increase the possibility of organisms' survival.

Moreover, I found that autophagy, apoptotic and sleep have a part-overlapped pathway in the regulation of larval survival during starvation. CEP-1, one of the essential proteins in germline apoptosis, might act in upstream in sleep pathway to inhibit APTF-1 and, thus, plays a negative role in L1-survival during starvation. It is consistent with the result shown in the previous study that down-regulating the cep- 1 expression is able to extend lifespan in the adult worms [198]. Unexpectedly, knocking out of the gene cep-1 did not increase the sleep of arrested L1 larvae. However, we did not have any evidence that overexpressing aptf-1 is able to increase sleep in arrested L1 larvae during starvation. Thus, it seems that the mechanisms of apoptosis in germline can only be associated with the protective program of sleep, but not with the sleep-active/sleep-promoting mechanism for the alteration of sleep-behavior in the starved larvae. In this study, it has also been proved that sleep is not a requirement of germline quiescence during the development period. This further supports that mechanisms of germline alteration are related to the functional mechanisms of sleep but not the sleep behavior in larvae during starvation. 


\subsection{Sleep and protein homeostasis}

Disruption of protein homeostasis, namely proteostasis, is a signal of aging. Aged worms show an increased aggregation of endogenous proteins $[171,199,200]$. Sleep seems to be able to inhibit or counteract protein aggregation and play a role in protein homeostasis. Heat shock proteins (HSPs) are required for protein homeostasis. They destabilize protein aggregation, guard protein folding and sorting in the right way [201].

In $C$. elegans, HSPs are induced by stresses and accumulate during aging [179, 202, 203]. It has been shown that the expression level of HSPs is associated with aging and longevity [204]. Several genes that regulate the expression of HSPs increase or decrease the lifespan of adult worms. Interestingly, in this study, HSP-4 cannot be appropriately expressed and accumulated during aging in sleep-deprived larvae. Moreover, without sleep, a decreased expression of HSP-16.2 was found in the arrested L1 larvae in response to heat shock stress. These results indicate that sleep affects the expression of HSPs and thus play a promotional role in the stress response mechanism and the aging process in C.elegans. Because of the inseparable connection between stress responses and protein homeostasis, it could be hypothesized that sleep might be able to control aging through regulating protein homeostasis in C.elegans during starvation, especially in the arrested L1 larvae, which do not contain the other complete protective mechanisms yet. However, further investigations are required to examine the effects of sleep in the proteostasis-declined mutant and explore the mechanisms behind sleep, protein homeostasis and aging in C. elegans.

\subsection{Sleep and mitochondrial respiratory chain}

It is well known that mitochondrial dysfunction is associated with aging [205]. The physical declines in old adults are mainly caused by sarcopenia and heart failure, which are the two consequences of mitochondrial dysfunction [206]. Sarcopenia is a decline in muscle mass related to aging. It is observed together with mitochondrial defections in body wall muscles of aged adult $C$. elegans [207]. Thus, declined muscle structure and defected mitochondria such as mitochondrial fragmentation were considered as the biomarkers for aging. Both phenotypes occur in the aged adult worms and the L1 larvae 
arrested after a long period. Interestingly, sleep is able to counteract both aging phenotypes. Because the changes in mitochondrial structure are due to the mitochondrial dysfunction [116], sleep thus might be related to the functions of mitochondria which impact aging and survival in arrested L1 larvae.

The MRC synthesizes ATP by the action of complexes and links directly to the energy hemostasis in response to starvation. Indeed, I found that mutations of MRC complex I, II and III reduce the harm caused by sleep deprivation at the expense of slowing down the development process in arrested L1 larvae during starvation. According to these encouraging results, it is possible to hypothesize that MRC may act in the functional downstream pathway of sleep to promote survival and slow down the aging process in arrested L1 during starvation. Moreover, MRC complex III is further involved in the behavior-regulatory pathway of sleep.

To further suppose the hypothesis, I measured ROS and redox level in arrested L1 larvae. Even though ROS have multiple end- or exogenous sources, more than $90 \%$ of ROS occur as a by-product of MRC and link to the energy synthesis [208]. ROS have been considered as toxic products, which induce oxidative stress as well as damage, and are harmful to longevity for many years. However, the interaction between ROS and aging turns out to be much more complex than we previously thought. Increased number of evidence has showed that rather than the toxic byproducts, ROS act as a secondary messenger in antioxidant signaling [209]. High level of ROS production does not simply mean short lifespan, and it could also induce the adaptive mechanisms in response to high oxidative stress and protect organisms [210]. Moreover, it has been shown that increasing ROS productions are able to facilitate the mitochondrial functions and, thus, promote the health of the elderly $[211,212]$. Redox homeostasis and ROS react together to the age-induced damages. Alteration of redox circuit has been known to relate many diseases and neurodegenerative disorders, and it plays a vital role with ROS productions in regulating cell growth, apoptosis and aging [213].

In this study, sleep was found to increase the ROS level and the redox stage in arrested L1 throughout the survival during starvation. It indicates that sleep might have an overlapped pathway with the antioxidant signaling of ROS and altered redox 
homeostasis. Furthermore, The redox state in arrested L1 larvae provides consistent evidence that sleeping worms are more oxidized than the sleepless mutant L1 larvae during starvation, as described in pervious study [132]. In summary, all the results support the hypothesis above that MRC is involved in the functional pathway of sleep in response to stresses and promote survival as well as retarding aging by increasing ROS production in arrested L1 during starvation.

\subsection{Sleep might contain two partly distinct mechanisms}

Sleep is triggered by starvation through the activation of RIS neuron in C. elegans. This sleep-active/sleep-promoting pathway was identified and is controlled by FoxO and AMPK in parallel. Without sleep, arrested L1 larvae showed decreased survival and accelerated aging. I thus questioned whether an increase of sleep behavior or upregulating of sleep-active signaling is able to benefit survival and slow down the aging process in arrested L1 during starvation. Unexpectedly, simply increasing the sleep behavior did not contribute to the survival rate. No extension of survival was found in the sleepiness of L1 larvae during starvation. Moreover, overexpressing flp-11 increased neither sleep fraction nor larval survival in arrested L1 worms during starvation. Nevertheless, these negative results might be attributed to inadequate research design [214].

Since the findings in my study indicate that energy exhaustion is not the major reason for the premature death in starved L1 larvae, I can infer that the underlying mechanism of sleep for survival extension is partly distinct from the sleep-activating/sleep-promoting mechanisms in arrested L1 larvae. Activation of RIS might further induce two different signaling. One is responsible for activation of sleep behavior to conserve energy; the other one is in charge of physiological benefits such as an increase in stress residence, retarding of aging process and extension of longevity. This hypothesis is consistent with the conclusion described in the paper above that sleep is required for starvation survival not only because it could save energy but also because it could counteract the aging process simultaneously [118]. 
Now we may be curious about the underlying mechanisms of sleep that control sleep behavior or benefits the survival span, and retard aging during starvation. To answer this question, I did two screens in this study: mutation screen of behavioral alteration and mutation screen of survival alteration in arrested larvae during starvation. Masurat and I found that FoxO and AMPK control the sleep-active/sleep-promoting mechanism through IIS and sirtuin signaling in multiple tissues, but outside of RIS. The AMPK, PTEN as well as TOR signaling were found to regulate the functional mechanisms of the protective program of sleep in the arrested L1 larvae during starvation. Moreover, CEP-1 and MRC are also involved in this protective program of sleep.

One unanticipated finding was that ethanol seems to play a role in the protective program of sleep. With the addition of ethanol at a low concentration, the sleepless larvae are able to survive for almost the same time as the sleeping larvae. I questioned whether this "rescue" effect comes from an increase in energy supply because ethanol has been considered as a food source for C. elegans. Nevertheless, the starved wild-type L1 larvae did not show any extension of survival with ethanol at the same concentration. This initially reveals that ethanol did not simply serve as a food source to contribute to survival during L1 starvation. Although ethanol has shown no evidence in this study that it is able to influence sleep behavior in the arrested L1 larvae, it does participate in the mechanisms of the protective program of sleep. It has been reported that the chronic exposure of ethanol resulted in small body size and slow reproductive fecundity of the adult C. elegans [215]. In the arrested larvae, we also found that ethanol further impairs the development and the ability to re-enter the developmental circle from starvation. These observations point to the likelihood that ethanol is able to activate an unexplored pathway that is conducive to survival, but it might also compromise the developmental mechanisms in larval C. elegans. The most exciting thing is that this concealed pathway is tightly coupled with the protective program of sleep during starvation.

The results of my study have thrown up many questions in need of further investigations of sleep. Homeostatic balancing is a fundamental issue for organisms, and since sleep might play a vital role in the homeostasis and offers such incredible benefits to worms, the highly sophisticated and complicated molecular mechanisms of sleep should be firstly clarified in the future investigations. At this point, my study provides several 
unique insights into the following research directions of sleep functions, such as the mitochondrial respiratory chain, the protein homeostasis and the mechanisms of apoptosis. Furthermore, the observations in my study bring up another vital question: If the living organisms are homeostatic, what does sleep sacrifice for contributing survival in the larvae during starvation? I believe this further understanding could eventually give us a full picture of the mysterious functions not only of the worm sleep but also of the sleep in mammals. 


\section{Abbreviations}

\section{AMI}

AMPK

APTF-1

C. elegans

C1-BODIPY-C12

cAMP

Clock

CoQ

DHE

DIC

DMSO

DNA

DTS

EEG

EGF

EGFR

EM gain

ER

ETC

FLP

FoxO

GABA

GAL
Agarose microchamber imaging

5' adenosine monophosphate-activated protein kinase

AP-2 Transcription Factor-1

Caenorhabditis elegans

4,4-difluoro-5-methyl-4-bora-3a, 4a-diaza-s-indacene-3dodecanoic acid

Cyclic adenosine 3', 5'-monophosphate

Circadian locomotor output cycles kaput

Coenzyme Q10

Dihydroethidium

Differential interference contrast

Dimethyl sulfoxide

Deoxyribonucleic acid

Developmentally timed sleep

Electroencephalography

Epidermal growth factor

Epidermal growth factor receptor

Electron-multiplying gain

Endoplasmic reticulum

Mitochondrial electron transport chain

FMRFamide like peptides

Factor forkhead box $\mathrm{O}$

$\gamma$-aminobutyric acid

Galanin 
GFP

HSP

IGF-1

IIS

IPTG

L1-L4

LC

LED

MRC

NAD

NGM

NREM

PCR

PDF

PI

PIP3

PKA

PKG

Poly-Q

PTEN

Redox

REM

RNAi

ROS

SE
Green fluorescent protein

Heat shock protein

Insulin-like growth factor-1

Insulin/insulin-like growth factor-1 signaling

Isopropyl- $\beta$-d-thiogalactopyranoside

Larval stages 1-4

Locus coeruleus

Light-emitting diode

Mitochondrial respiratory chain

Nicotinamide adenine dinucleotide

Nematode growth medium

Non-rapid eye movement

Polymerase chain reaction

Pigment dispersing factor

Phosphoinositide

Phosphatidylinositol $(3,4,5)$-trisphosphate

Proteinkinase A

Proteinkinase G

Polyglutamine

Phosphatase and tensin homologue deleted on chromosome 10

Reduction-oxidation reaction

Rapid eye movement

RNA interference

Reactive oxygen species

Standard Error 
SEM

SIS

TGF $\beta$

TMN

TOR

TORC

UPR

VLPO
Standard Error of the Mean

Stress-induced sleep

Transforming growth factor $\beta$

Tuberomammillary nucleus

Target of rapamycin

Target of rapamycin complex

Unfolded protein response

Ventrolateral preoptic nucleus 


\section{References}

1.

2.

3.

4.

5.

6.

7.

8.

9.

10.

11.

12.

13.

14.

15.

16.

17.

18.

Siegel, J.M., Do all animals sleep? Trends Neurosci, 2008. 31(4): p. 208-13.

Campbell, S.S. and I. Tobler, Animal sleep: a review of sleep duration across phylogeny. Neurosci Biobehav Rev, 1984. 8(3): p. 269-300.

Lesku, J.A., et al., A phylogenetic analysis of sleep architecture in mammals: the integration of anatomy, physiology, and ecology. Am Nat, 2006. 168(4): p. 441-53.

Siegel, J.M., Clues to the functions of mammalian sleep. Nature, 2005. 437(7063): p. 1264-71.

Gravett, N., et al., Inactivity/sleep in two wild free-roaming African elephant matriarchs - Does large body size make elephants the shortest mammalian sleepers? PLoS One, 2017. 12(3): p. e0171903.

Flanigan, W.F., Jr., Sleep and wakefulness in iguanid lizards, Ctenosaura pectinata and Iguana iguana. Brain Behav Evol, 1973. 8(6): p. 401-36.

Loomis, A.L., E.N. Harvey, and G. Hobart, Further Observations on the Potential Rhythms of the Cerebral Cortex during Sleep. Science, 1935. 82(2122): p. 198-200.

Aserinsky, E. and N. Kleitman, Regularly occurring periods of eye motility, and concomitant phenomena, during sleep. Science, 1953. 118(3062): p. 273-4.

Miyazaki, S., C.Y. Liu, and Y. Hayashi, Sleep in vertebrate and invertebrate animals, and insights into the function and evolution of sleep. Neurosci Res, 2017. 118: p. 3-12.

Raizen, D.M., et al., Lethargus is a Caenorhabditis elegans sleep-like state. Nature, 2008. 451(7178): p. 569-72.

Hill, A.J., et al., Cellular stress induces a protective sleep-like state in C. elegans. Curr Biol, 2014. 24(20): p. 2399-405.

Turek, M., I. Lewandrowski, and H. Bringmann, An AP2 transcription factor is required for a sleep-active neuron to induce sleep-like quiescence in C. elegans. Curr Biol, 2013. 23(22): p. 2215-2223.

Hendricks, J.C., et al., Rest in Drosophila is a sleep-like state. Neuron, 2000. 25(1): p. 129-38.

Shaw, P.J., et al., Correlates of sleep and waking in Drosophila melanogaster. Science, 2000. 287(5459): p. 1834-7.

Zhdanova, I.V., et al., Melatonin promotes sleep-like state in zebrafish. Brain Res, 2001. 903(1-2): p. 263-8.

Saper, C.B., T.C. Chou, and T.E. Scammell, The sleep switch: hypothalamic control of sleep and wakefulness. Trends Neurosci, 2001. 24(12): p. 726-31.

Saper, C.B., T.E. Scammell, and J. Lu, Hypothalamic regulation of sleep and circadian rhythms. Nature, 2005. 437(7063): p. 1257-63.

Sherin, J.E., et al., Activation of ventrolateral preoptic neurons during sleep. Science, 1996. 271(5246): p. 216-9. 
19.

20.

21.

22.

23.

24.

25.

26.

27.

28.

30.

31.

33.

34.

35.

36.

37.

38.

Sherin, J.E., et al., Innervation of histaminergic tuberomammillary neurons by GABAergic and galaninergic neurons in the ventrolateral preoptic nucleus of the rat. $\mathrm{J}$ Neurosci, 1998. 18(12): p. 4705-21.

Gaus, S.E., et al., Ventrolateral preoptic nucleus contains sleep-active, galaninergic neurons in multiple mammalian species. Neuroscience, 2002. 115(1): p. 285-94.

Inutsuka, A. and A. Yamanaka, The regulation of sleep and wakefulness by the hypothalamic neuropeptide orexin/hypocretin. Nagoya J Med Sci, 2013. 75(1-2): p. 2936 .

Fisher, S.P., R.G. Foster, and S.N. Peirson, The circadian control of sleep. Handb Exp Pharmacol, 2013(217): p. 157-83.

Franken, P., A role for clock genes in sleep homeostasis. Curr Opin Neurobiol, 2013. 23(5): p. 864-72.

Alam, M.A., et al., Neuronal activity in the preoptic hypothalamus during sleep deprivation and recovery sleep. J Neurophysiol, 2014. 111(2): p. 287-99.

Cirelli, C. and G. Tononi, Is sleep essential? PLoS Biol, 2008. 6(8): p. e216.

Knutson, K.L., et al., The metabolic consequences of sleep deprivation. Sleep Med Rev, 2007. 11(3): p. 163-78.

Cirelli, C., C.M. Gutierrez, and G. Tononi, Extensive and divergent effects of sleep and wakefulness on brain gene expression. Neuron, 2004. 41(1): p. 35-43.

Seibt, J., et al., Protein synthesis during sleep consolidates cortical plasticity in vivo. Curr Biol, 2012. 22(8): p. 676-82.

de Vivo, L., et al., Ultrastructural evidence for synaptic scaling across the wake/sleep cycle. Science, 2017. 355(6324): p. 507-510.

Walker, M.P. and R. Stickgold, Sleep-dependent learning and memory consolidation. Neuron, 2004. 44(1): p. 121-33.

Dudai, Y., A. Karni, and J. Born, The Consolidation and Transformation of Memory. Neuron, 2015. 88(1): p. 20-32.

Peever, J. and P.M. Fuller, The Biology of REM Sleep. Curr Biol, 2017. 27(22): p. R1237-R1248.

Crocker, A. and A. Sehgal, Genetic analysis of sleep. Genes Dev, 2010. 24(12): p. 122035 .

Besedovsky, L., T. Lange, and J. Born, Sleep and immune function. Pflugers Arch, 2012. 463(1): p. 121-37.

Kayser, M.S. and D. Biron, Sleep and Development in Genetically Tractable Model Organisms. Genetics, 2016. 203(1): p. 21-33.

Krueger, J.M., et al., Sleep function: Toward elucidating an enigma. Sleep Med Rev, 2016. 28: p. 46-54.

Brenner, S., The genetics of Caenorhabditis elegans. Genetics, 1974. 77(1): p. 71-94.

Madl, J.E. and R.K. Herman, Polyploids and sex determination in Caenorhabditis elegans. Genetics, 1979. 93(2): p. 393-402. 
39.

40.

41.

42.

43.

44.

45.

46.

47.

48.

49.

50.

51.

52.

53.

54.

55.

56.

Golden, J.W. and D.L. Riddle, The Caenorhabditis elegans dauer larva: developmental effects of pheromone, food, and temperature. Dev Biol, 1984. 102(2): p. 368-78.

Baugh, L.R., To grow or not to grow: nutritional control of development during Caenorhabditis elegans L1 arrest. Genetics, 2013. 194(3): p. 539-55.

Consortium, C.e.S., Genome sequence of the nematode C. elegans: a platform for investigating biology. Science, 1998. 282(5396): p. 2012-8.

Sulston, J.E. and H.R. Horvitz, Post-embryonic cell lineages of the nematode, Caenorhabditis elegans. Dev Biol, 1977. 56(1): p. 110-56.

White, J.G., et al., The structure of the nervous system of the nematode Caenorhabditis elegans. Philos Trans R Soc Lond B Biol Sci, 1986. 314(1165): p. 1-340.

Hardin, P.E., J.C. Hall, and M. Rosbash, Feedback of the Drosophila period gene product on circadian cycling of its messenger RNA levels. Nature, 1990. 343(6258): p. 536-40.

Sun, Z.S., et al., RIGUI, a putative mammalian ortholog of the Drosophila period gene. Cell, 1997. 90(6): p. 1003-11.

Tei, H., et al., Circadian oscillation of a mammalian homologue of the Drosophila period gene. Nature, 1997. 389(6650): p. 512-6.

Choi, S., et al., Analysis of NPR-1 reveals a circuit mechanism for behavioral quiescence in C. elegans. Neuron, 2013. 78(5): p. 869-80.

Van Buskirk, C. and P.W. Sternberg, Epidermal growth factor signaling induces behavioral quiescence in Caenorhabditis elegans. Nat Neurosci, 2007. 10(10): p. 13007.

Iwanir, S., et al., The microarchitecture of C. elegans behavior during lethargus: homeostatic bout dynamics, a typical body posture, and regulation by a central neuron. Sleep, 2013. 36(3): p. 385-95.

Singh, K., et al., Deep conservation of genes required for both Drosphila melanogaster and Caenorhabditis elegans sleep includes a role for dopaminergic signaling. Sleep, 2014. 37(9): p. 1439-51.

Belfer, S.J., et al., Caenorhabditis-in-Drop Array for Monitoring C. elegans Quiescent Behavior. Sleep, 2013. 36(5): p. 689-698.

Jones, D. and E.P. Candido, Feeding is inhibited by sublethal concentrations of toxicants and by heat stress in the nematode Caenorhabditis elegans: relationship to the cellular stress response. J Exp Zool, 1999. 284(2): p. 147-57.

Nelson, M.D., et al., FMRFamide-like FLP-13 neuropeptides promote quiescence following heat stress in Caenorhabditis elegans. Curr Biol, 2014. 24(20): p. 2406-10.

Schwarz, J., I. Lewandrowski, and H. Bringmann, Reduced activity of a sensory neuron during a sleep-like state in Caenorhabditis elegans. Curr Biol, 2011. 21(24): p. R983-4.

Negy, C., et al., Psychological homelessness and enculturative stress among USdeported Salvadorans: a preliminary study with a novel approach. J Immigr Minor Health, 2014. 16(6): p. 1278-83.

Cho, J.Y. and P.W. Sternberg, Multilevel modulation of a sensory motor circuit during C. elegans sleep and arousal. Cell, 2014. 156(1-2): p. 249-60. 
57.

58.

59.

60.

61.

64.

65.

66.

67.

68.

69.

70.

71.

73.

74

Nelson, M.D., et al., The neuropeptide NLP-22 regulates a sleep-like state in Caenorhabditis elegans. Nat Commun, 2013. 4: p. 2846.

Turek, M., et al., Sleep-active neuron specification and sleep induction require FLP-11 neuropeptides to systemically induce sleep. Elife, 2016. 5.

You, Y.J., et al., Insulin, $c G M P$, and TGF-beta signals regulate food intake and quiescence in C. elegans: a model for satiety. Cell Metab, 2008. 7(3): p. 249-57.

Gallagher, T., et al., ASI regulates satiety quiescence in C. elegans. J Neurosci, 2013. 33(23): p. 9716-24.

Mani, A., et al., Syndromic patent ductus arteriosus: evidence for haploinsufficient TFAP2B mutations and identification of a linked sleep disorder. Proc Natl Acad Sci U S A, 2005. 102(8): p. 2975-9.

Kucherenko, M.M., et al., TfAP-2 is required for night sleep in Drosophila. BMC Neurosci, 2016. 17(1): p. 72.

Kenyon, C., The first long-lived mutants: discovery of the insulin/IGF-1 pathway for ageing. Philos Trans R Soc Lond B Biol Sci, 2011. 366(1561): p. 9-16.

Kenyon, C., et al., A C. elegans mutant that lives twice as long as wild type. Nature, 1993. 366(6454): p. 461-4.

Kimura, K.D., et al., daf-2, an insulin receptor-like gene that regulates longevity and diapause in Caenorhabditis elegans. Science, 1997. 277(5328): p. 942-6.

Friedman, D.B. and T.E. Johnson, A mutation in the age-1 gene in Caenorhabditis elegans lengthens life and reduces hermaphrodite fertility. Genetics, 1988. 118(1): p. 75-86.

Friedman, D.B. and T.E. Johnson, Three mutants that extend both mean and maximum life span of the nematode, Caenorhabditis elegans, define the age-1 gene. J Gerontol, 1988. 43(4): p. B102-9.

Ogg, S., et al., The Fork head transcription factor DAF-16 transduces insulin-like metabolic and longevity signals in C. elegans. Nature, 1997. 389(6654): p. 994-9.

Lin, K., et al., daf-16: An HNF-3/forkhead family member that can function to double the life-span of Caenorhabditis elegans. Science, 1997. 278(5341): p. 1319-22.

Murphy, C.T., et al., Genes that act downstream of DAF-16 to influence the lifespan of Caenorhabditis elegans. Nature, 2003. 424(6946): p. 277-83.

McElwee, J., K. Bubb, and J.H. Thomas, Transcriptional outputs of the Caenorhabditis elegans forkhead protein DAF-16. Aging Cell, 2003. 2(2): p. 111-21.

McElwee, J.J., et al., Shared transcriptional signature in Caenorhabditis elegans Dauer larvae and long-lived daf-2 mutants implicates detoxification system in longevity assurance. J Biol Chem, 2004. 279(43): p. 44533-43.

Halaschek-Wiener, J., et al., Analysis of long-lived C. elegans daf-2 mutants using serial analysis of gene expression. Genome Res, 2005. 15(5): p. 603-15.

Ogg, S. and G. Ruvkun, The C. elegans PTEN homolog, DAF-18, acts in the insulin receptor-like metabolic signaling pathway. Mol Cell, 1998. 2(6): p. 887-93. 
75.

76.

77.

78.

79.

80.

81.

82.

83.

84.

85.

86.

87.

88.

89.

90.

91.

92.

Oh, S.W., et al., JNK regulates lifespan in Caenorhabditis elegans by modulating nuclear translocation of forkhead transcription factor/DAF-16. Proc Natl Acad Sci U S A, 2005. 102(12): p. 4494-9.

Lehtinen, M.K., et al., A conserved MST-FOXO signaling pathway mediates oxidativestress responses and extends life span. Cell, 2006. 125(5): p. 987-1001.

Li, W., et al., RLE-1, an E3 ubiquitin ligase, regulates $C$. elegans aging by catalyzing DAF-16 polyubiquitination. Dev Cell, 2007. 12(2): p. 235-46.

Takahashi, Y., et al., Asymmetric arginine dimethylation determines life span in $C$. elegans by regulating forkhead transcription factor DAF-16. Cell Metab, 2011. 13(5): p. 505-16.

Loewith, R. and M.N. Hall, Target of rapamycin (TOR) in nutrient signaling and growth control. Genetics, 2011. 189(4): p. 1177-201.

Wullschleger, S., R. Loewith, and M.N. Hall, TOR signaling in growth and metabolism. Cell, 2006. 124(3): p. 471-84.

Vellai, T., et al., Genetics: influence of TOR kinase on lifespan in C. elegans. Nature, 2003. 426(6967): p. 620.

Jia, K., D. Chen, and D.L. Riddle, The TOR pathway interacts with the insulin signaling pathway to regulate C. elegans larval development, metabolism and life span.

Development, 2004. 131(16): p. 3897-906.

Hsu, A.L., C.T. Murphy, and C. Kenyon, Regulation of aging and age-related disease by DAF-16 and heat-shock factor. Science, 2003.300(5622): p. 1142-5.

Chiang, W.C., et al., HSF-1 regulators DDL-1/2 link insulin-like signaling to heat-shock responses and modulation of longevity. Cell, 2012. 148(1-2): p. 322-34.

Zhang, Y., et al., The HIF-1 hypoxia-inducible factor modulates lifespan in C. elegans. PLoS One, 2009. 4(7): p. e6348.

Leiser, S.F., et al., Life-span extension from hypoxia in Caenorhabditis elegans requires both HIF-1 and DAF-16 and is antagonized by SKN-1. J Gerontol A Biol Sci Med Sci, 2013. 68(10): p. 1135-44.

Sheaffer, K.L., D.L. Updike, and S.E. Mango, The Target of Rapamycin pathway antagonizes pha-4/FoxA to control development and aging. Curr Biol, 2008. 18(18): p. 1355-64.

Panowski, S.H., et al., PHA-4/Foxa mediates diet-restriction-induced longevity of C. elegans. Nature, 2007. 447(7144): p. 550-5.

Tullet, J.M., et al., Direct inhibition of the longevity-promoting factor SKN-1 by insulinlike signaling in C. elegans. Cell, 2008. 132(6): p. 1025-38.

Lee, S.J., A.B. Hwang, and C. Kenyon, Inhibition of respiration extends C. elegans life span via reactive oxygen species that increase HIF-1 activity. Curr Biol, 2010. 20(23): p. 2131-6.

Hardie, D.G., AMP-activated protein kinase: an energy sensor that regulates all aspects of cell function. Genes Dev, 2011. 25(18): p. 1895-908.

Apfeld, J., et al., The AMP-activated protein kinase AAK-2 links energy levels and insulin-like signals to lifespan in C. elegans. Genes Dev, 2004. 18(24): p. 3004-9. 
93.

94.

95.

96.

97.

98.

99.

100.

101.

102.

103.

104.

105.

106.

107.

108.

109.

110.

Greer, E.L., et al., An AMPK-FOXO pathway mediates longevity induced by a novel method of dietary restriction in C. elegans. Curr Biol, 2007. 17(19): p. 1646-56.

Mair, W., et al., Lifespan extension induced by AMPK and calcineurin is mediated by CRTC-1 and CREB. Nature, 2011. 470(7334): p. 404-8.

Preyat, N. and O. Leo, Sirtuin deacylases: a molecular link between metabolism and immunity. J Leukoc Biol, 2013. 93(5): p. 669-80.

Tissenbaum, H.A. and L. Guarente, Increased dosage of a sir-2 gene extends lifespan in Caenorhabditis elegans. Nature, 2001. 410(6825): p. 227-30.

Berdichevsky, A., et al., C. elegans SIR-2.1 interacts with 14-3-3 proteins to activate DAF-16 and extend life span. Cell, 2006. 125(6): p. 1165-77.

Curtis, R., G. O'Connor, and P.S. DiStefano, Aging networks in Caenorhabditis elegans: AMP-activated protein kinase (aak-2) links multiple aging and metabolism pathways. Aging Cell, 2006. 5(2): p. 119-26.

Wang, Y. and H.A. Tissenbaum, Overlapping and distinct functions for a Caenorhabditis elegans SIR2 and DAF-16/FOXO. Mech Ageing Dev, 2006. 127(1): p. 48-56.

Lee, G.D., et al., Dietary deprivation extends lifespan in Caenorhabditis elegans. Aging Cell, 2006. 5(6): p. 515-24.

Greer, E.L. and A. Brunet, Different dietary restriction regimens extend lifespan by both independent and overlapping genetic pathways in C. elegans. Aging Cell, 2009. 8(2): p. 113-27.

Harman, D., Aging: a theory based on free radical and radiation chemistry. J Gerontol, 1956. 11(3): p. 298-300.

Dillin, A., et al., Rates of behavior and aging specified by mitochondrial function during development. Science, 2002. 298(5602): p. 2398-401.

Hamilton, B., et al., A systematic RNAi screen for longevity genes in C. elegans. Genes Dev, 2005. 19(13): p. 1544-55.

Lee, S.S., et al., A systematic RNAi screen identifies a critical role for mitochondria in C. elegans longevity. Nat Genet, 2003. 33(1): p. 40-8.

Hansen, M., et al., New genes tied to endocrine, metabolic, and dietary regulation of lifespan from a Caenorhabditis elegans genomic RNAi screen. PLoS Genet, 2005. 1(1): p. 119-28.

Durieux, J., S. Wolff, and A. Dillin, The cell-non-autonomous nature of electron transport chain-mediated longevity. Cell, 2011. 144(1): p. 79-91.

Mouchiroud, L., et al., The NAD(+)/Sirtuin Pathway Modulates Longevity through Activation of Mitochondrial UPR and FOXO Signaling. Cell, 2013. 154(2): p. 430-41.

Kong, Y., S.E. Trabucco, and H. Zhang, Oxidative stress, mitochondrial dysfunction and the mitochondria theory of aging. Interdiscip Top Gerontol, 2014. 39: p. 86-107.

Gems, D. and R. Doonan, Antioxidant defense and aging in C. elegans: is the oxidative damage theory of aging wrong? Cell Cycle, 2009. 8(11): p. 1681-7. 
111. Van Raamsdonk, J.M. and S. Hekimi, Superoxide dismutase is dispensable for normal animal lifespan. Proc Natl Acad Sci U S A, 2012. 109(15): p. 5785-90.

112. Van Raamsdonk, J.M. and S. Hekimi, Deletion of the mitochondrial superoxide dismutase sod-2 extends lifespan in Caenorhabditis elegans. PLoS Genet, 2009. 5(2): p. e1000361.

113. Baruah, A., et al., CEP-1, the Caenorhabditis elegans p53 homolog, mediates opposing longevity outcomes in mitochondrial electron transport chain mutants. PLoS Genet, 2014. 10(2): p. e1004097.

114. Hwang, A.B., et al., Feedback regulation via AMPK and HIF-1 mediates ROSdependent longevity in Caenorhabditis elegans. Proc Natl Acad Sci U S A, 2014. 111(42): p. E4458-67.

115. Schmeisser, S., et al., Mitochondrial hormesis links low-dose arsenite exposure to lifespan extension. Aging Cell, 2013. 12(3): p. 508-17.

Gaffney, C.J., et al., Greater loss of mitochondrial function with ageing is associated with earlier onset of sarcopenia in C. elegans. Aging (Albany NY), 2018. 10(11): p. 3382-3396.

Roux, A.E., et al., Reversible Age-Related Phenotypes Induced during Larval Quiescence in C. elegans. Cell Metab, 2016. 23(6): p. 1113-1126.

Wu, Y., et al., Sleep Counteracts Aging Phenotypes to Survive Starvation-Induced Developmental Arrest in C. elegans. Curr Biol, 2018. 28(22): p. 3610-3624 e8.

Bringmann, H., Agarose hydrogel microcompartments for imaging sleep-and wake-like behavior and nervous system development in Caenorhabditis elegans larvae. J Neurosci Methods, 2011. 201(1): p. 78-88.

Turek, M., J. Besseling, and H. Bringmann, Agarose Microchambers for Long-term Calcium Imaging of Caenorhabditis elegans. J Vis Exp, 2015(100): p. e52742.

Nagy, S., D.M. Raizen, and D. Biron, Measurements of behavioral quiescence in Caenorhabditis elegans. Methods, 2014. 68(3): p. 500-7.

Lewis, J.A. and J.T. Fleming, Basic culture methods. Methods Cell Biol, 1995. 48: p. 329.

Kaeberlein, T.L., et al., Lifespan extension in Caenorhabditis elegans by complete removal of food. Aging Cell, 2006. 5(6): p. 487-94.

Uno, M., et al., A fasting-responsive signaling pathway that extends life span in $C$. elegans. Cell Rep, 2013. 3(1): p. 79-91.

Kamath, R.S. and J. Ahringer, Genome-wide RNAi screening in Caenorhabditis elegans. Methods, 2003. 30(4): p. 313-21.

Timmons, L., D.L. Court, and A. Fire, Ingestion of bacterially expressed dsRNAs can produce specific and potent genetic interference in Caenorhabditis elegans. Gene, 2001. 263(1-2): p. 103-12.

Goodman, L., On the Exact Variance of Products. Journal of the American Statistical Association, 1960. 55(292): p. 6.

Stiernagle, T., Maintenance of C. elegans. WormBook, 2006: p. 1-11. 
129.

130.

131.

133.

134.

135.

136.

137.

138.

139.

140.

141.

142.

143.

144.

145.

146.

Kasurinen, J., A novel fluorescent fatty acid, 5-methyl-BDY-3-dodecanoic acid, is a potential probe in lipid transport studies by incorporating selectively to lipid classes of BHK cells. Biochem Biophys Res Commun, 1992. 187(3): p. 1594-601.

Spike, C.A., et al., DEPS-1 promotes $P$-granule assembly and RNA interference in $C$. elegans germ cells. Development, 2008. 135(5): p. 983-93.

Mello, C.C., et al., The PIE-1 protein and germline specification in C. elegans embryos. Nature, 1996. 382(6593): p. 710-2.

Kirstein, J., et al., Proteotoxic stress and ageing triggers the loss of redox homeostasis across cellular compartments. EMBO J, 2015. 34(18): p. 2334-49.

Crane, R.K. and A. Sols, The non-competitive inhibition of brain hexokinase by glucose6-phosphate and related compounds. J Biol Chem, 1954. 210(2): p. 597-606.

Schulz, T.J., et al., Glucose restriction extends Caenorhabditis elegans life span by inducing mitochondrial respiration and increasing oxidative stress. Cell Metab, 2007. 6(4): p. 280-93.

Lee, S.J., C.T. Murphy, and C. Kenyon, Glucose shortens the life span of C. elegans by downregulating DAF-16/FOXO activity and aquaporin gene expression. Cell Metab, 2009. 10(5): p. 379-91.

Massie, M.R., et al., Exposure to the metabolic inhibitor sodium azide induces stress protein expression and thermotolerance in the nematode Caenorhabditis elegans. Cell Stress \& Chaperones, 2003. 8(1): p. 1-7.

Takauji, Y., et al., Restriction of protein synthesis abolishes senescence features at cellular and organismal levels. Sci Rep, 2016. 6: p. 18722.

Ennis, H.L. and M. Lubin, Cycloheximide: Aspects of Inhibition of Protein Synthesis in Mammalian Cells. Science, 1964. 146(3650): p. 1474-6.

Robida-Stubbs, S., et al., TOR signaling and rapamycin influence longevity by regulating $S K N-1 / N r f$ and DAF-16/FoxO. Cell Metab, 2012. 15(5): p. 713-24.

Fontana, L., L. Partridge, and V.D. Longo, Extending healthy life span--from yeast to humans. Science, 2010. 328(5976): p. 321-6.

Jones, K.T., et al., Rictor/TORC2 regulates Caenorhabditis elegans fat storage, body size, and development through sgk-1. PLoS Biol, 2009. 7(3): p. e60.

Kim, E., et al., Regulation of TORC1 by Rag GTPases in nutrient response. Nat Cell Biol, 2008. 10(8): p. 935-45.

Schreiber, M.A., et al., Manipulation of behavioral decline in Caenorhabditis elegans with the Rag GTPase raga-1. PLoS Genet, 2010. 6(5): p. e1000972.

Gatsi, R., et al., Prohibitin-mediated lifespan and mitochondrial stress implicate SGK-1, insulin/IGF and mTORC2 in C. elegans. PLoS One, 2014. 9(9): p. e107671.

Soukas, A.A., et al., Rictor/TORC2 regulates fat metabolism, feeding, growth, and life span in Caenorhabditis elegans. Genes Dev, 2009. 23(4): p. 496-511.

Schumacher, B., et al., The C. elegans homolog of the p53 tumor suppressor is required for DNA damage-induced apoptosis. Curr Biol, 2001. 11(21): p. 1722-7. 
147. Derry, W.B., et al., Regulation of developmental rate and germ cell proliferation in Caenorhabditis elegans by the p53 gene network. Cell Death Differ, 2007. 14(4): p. 66270.

148. Greiss, S., et al., Transcriptional profiling in C. elegans suggests DNA damage dependent apoptosis as an ancient function of the p53 family. BMC Genomics, 2008. 9: p. 334.

149. Leprivier, G., et al., The eEF2 kinase confers resistance to nutrient deprivation by blocking translation elongation. Cell, 2013. 153(5): p. 1064-79.

150. Minnerly, J., et al., The cell non-autonomous function of ATG-18 is essential for neuroendocrine regulation of Caenorhabditis elegans lifespan. PLoS Genet, 2017. 13(5): p. e1006764.

151. Cabreiro, F., et al., Metformin retards aging in C. elegans by altering microbial folate and methionine metabolism. Cell, 2013. 153(1): p. 228-39.

Yang, W. and S. Hekimi, Two modes of mitochondrial dysfunction lead independently to lifespan extension in Caenorhabditis elegans. Aging Cell, 2010. 9(3): p. 433-47.

Ishii, N., et al., A methyl viologen-sensitive mutant of the nematode Caenorhabditis elegans. Mutat Res, 1990. 237(3-4): p. 165-71.

154. Ishii, N., et al., A mutation in succinate dehydrogenase cytochrome b causes oxidative stress and ageing in nematodes. Nature, 1998. 394(6694): p. 694-7.

Ishii, N., S. Goto, and P.S. Hartman, Protein oxidation during aging of the nematode Caenorhabditis elegans. Free Radic Biol Med, 2002. 33(8): p. 1021-5.

Battino, M., et al., Natural distribution and occurrence of coenzyme $Q$ homologues. Membr Biochem, 1990. 9(3): p. 179-90.

Tran, U.C. and C.F. Clarke, Endogenous synthesis of coenzyme $Q$ in eukaryotes. Mitochondrion, 2007. 7 Suppl: p. S62-71.

Lakowski, B. and S. Hekimi, Determination of life-span in Caenorhabditis elegans by four clock genes. Science, 1996. 272(5264): p. 1010-3.

Feng, J., F. Bussiere, and S. Hekimi, Mitochondrial electron transport is a key determinant of life span in Caenorhabditis elegans. Dev Cell, 2001. 1(5): p. 633-44.

Hirose, T., et al., Cyclic GMP-dependent protein kinase EGL-4 controls body size and lifespan in C elegans. Development, 2003. 130(6): p. 1089-99.

Van Voorhies, W.A., The influence of metabolic rate on longevity in the nematode Caenorhabditis elegans. Aging Cell, 2002. 1(2): p. 91-101.

Braeckman, B.P., et al., No reduction of energy metabolism in Clk mutants. Mech Ageing Dev, 2002. 123(11): p. 1447-56.

163.

Hartman, P.S., et al., Mitochondrial mutations differentially affect aging, mutability and anesthetic sensitivity in Caenorhabditis elegans. Mech Ageing Dev, 2001. 122(11): p. $1187-201$.

164.

Zarse, K., et al., Impaired insulin/IGF1 signaling extends life span by promoting mitochondrial L-proline catabolism to induce a transient ROS signal. Cell Metab, 2012. 15(4): p. 451-65. 
165.

166.

167.

168.

169.

170.

171.

172.

173.

174.

175.

176.

177.

178.

180.

181.

182.

183.

Yee, C., W. Yang, and S. Hekimi, The intrinsic apoptosis pathway mediates the prolongevity response to mitochondrial ROS in C. elegans. Cell, 2014. 157(4): p. 897-909.

Back, P., et al., Exploring real-time in vivo redox biology of developing and aging Caenorhabditis elegans. Free Radic Biol Med, 2012. 52(5): p. 850-9.

Carter, W.O., P.K. Narayanan, and J.P. Robinson, Intracellular hydrogen peroxide and superoxide anion detection in endothelial cells. J Leukoc Biol, 1994. 55(2): p. 253-8.

Balchin, D., M. Hayer-Hartl, and F.U. Hartl, In vivo aspects of protein folding and quality control. Science, 2016. 353(6294): p. aac4354.

Feleciano, D.R., K. Arnsburg, and J. Kirstein, Interplay between redox and protein homeostasis. Worm, 2016. 5(2): p. e1170273.

Ben-Zvi, A., E.A. Miller, and R.I. Morimoto, Collapse of proteostasis represents an early molecular event in Caenorhabditis elegans aging. Proc Natl Acad Sci U S A, 2009. 106(35): p. 14914-9.

David, D.C., et al., Widespread protein aggregation as an inherent part of aging in $C$. elegans. PLoS Biol, 2010. 8(8): p. e1000450.

Hanson, G.T., et al., Investigating mitochondrial redox potential with redox-sensitive green fluorescent protein indicators. J Biol Chem, 2004. 279(13): p. 13044-53.

Fukuyama, M., A.E. Rougvie, and J.H. Rothman, C. elegans DAF-18/PTEN mediates nutrient-dependent arrest of cell cycle and growth in the germline. Curr Biol, 2006. 16(8): p. 773-9.

Shin, H.S., et al., Endoplasmic reticulum stress as a novel target to ameliorate epithelial-to-mesenchymal transition and apoptosis of human peritoneal mesothelial cells. Lab Invest, 2015. 95(10): p. 1157-73.

Hayat, M.A., Autophagy : cancer, other pathologies, inflammation, immunity, infection, and aging. 2016, Amsterdam ;: Boston : Elsevier, Academic Press. volumes.

Taylor, R.C., Aging and the UPR(ER). Brain Res, 2016. 1648(Pt B): p. 588-593.

Hendershot, L.M., et al., Localization of the gene encoding human BiP/GRP78, the endoplasmic reticulum cognate of the HSP70 family, to chromosome 9q34. Genomics, 1994. 20(2): p. 281-4.

Walker, G.A. and G.J. Lithgow, Lifespan extension in C. elegans by a molecular chaperone dependent upon insulin-like signals. Aging Cell, 2003. 2(2): p. 131-9.

Rea, S.L., et al., A stress-sensitive reporter predicts longevity in isogenic populations of Caenorhabditis elegans. Nat Genet, 2005. 37(8): p. 894-8.

Fonte, V., et al., Suppression of in vivo beta-amyloid peptide toxicity by overexpression of the HSP-16.2 small chaperone protein. J Biol Chem, 2008. 283(2): p. 784-91.

Srinivasan, S., Regulation of body fat in Caenorhabditis elegans. Annu Rev Physiol, 2015. 77: p. 161-78.

Skora, S., F. Mende, and M. Zimmer, Energy Scarcity Promotes a Brain-wide Sleep State Modulated by Insulin Signaling in C. elegans. Cell Rep, 2018. 22(4): p. 953-966.

Schmidt, M.H., The energy allocation function of sleep: a unifying theory of sleep, torpor, and continuous wakefulness. Neurosci Biobehav Rev, 2014. 47: p. 122-53. 
184.

185.

186.

187.

188.

189.

190.

191.

192.

193.

194.

195.

196.

198.

199.

200.

201.

Kilduff, T.S., et al., Sleep and mammalian hibernation: homologous adaptations and homologous processes? Sleep, 1993. 16(4): p. 372-86.

Hu, P.J., Dauer. WormBook, 2007: p. 1-19.

Apfeld, J. and C. Kenyon, Regulation of lifespan by sensory perception in Caenorhabditis elegans. Nature, 1999. 402(6763): p. 804-9.

Lee, B.H. and K. Ashrafi, A TRPV channel modulates C. elegans neurosecretion, larval starvation survival, and adult lifespan. PLoS Genet, 2008. 4(10): p. e1000213.

Baugh, L.R. and P.W. Sternberg, DAF-16/FOXO regulates transcription of cki1/Cip/Kip and repression of lin-4 during C. elegans L1 arrest. Curr Biol, 2006. 16(8): p. 780-5.

Morley, J.F. and R.I. Morimoto, Regulation of longevity in Caenorhabditis elegans by heat shock factor and molecular chaperones. Mol Biol Cell, 2004. 15(2): p. 657-64.

Dijk, D.J. and J.F. Duffy, Circadian regulation of human sleep and age-related changes in its timing, consolidation and EEG characteristics. Ann Med, 1999. 31(2): p. 130-40.

Landolt, H.P. and A.A. Borbely, Age-dependent changes in sleep EEG topography. Clin Neurophysiol, 2001. 112(2): p. 369-77.

Landolt, H.P., et al., Effect of age on the sleep EEG: slow-wave activity and spindle frequency activity in young and middle-aged men. Brain Res, 1996. 738(2): p. 205-12.

Lord, C., Z. Sekerovic, and J. Carrier, Sleep regulation and sex hormones exposure in men and women across adulthood. Pathol Biol (Paris), 2014. 62(5): p. 302-10.

Vitiello, M.V., et al., Cognitive behavioral therapy for insomnia improves sleep and decreases pain in older adults with co-morbid insomnia and osteoarthritis. J Clin Sleep Med, 2009. 5(4): p. 355-62.

Mander, B.A., et al., Sleep: A Novel Mechanistic Pathway, Biomarker, and Treatment Target in the Pathology of Alzheimer's Disease? Trends Neurosci, 2016. 39(8): p. 552566.

Gump, J.M. and A. Thorburn, Autophagy and apoptosis: what is the connection? Trends Cell Biol, 2011. 21(7): p. 387-92.

Fan, Y.J. and W.X. Zong, The cellular decision between apoptosis and autophagy. Chin J Cancer, 2013. 32(3): p. 121-9.

Yanase, S., et al., Impaired p53/CEP-1 is associated with lifespan extension through an age-related imbalance in the energy metabolism of C. elegans. Genes Cells, 2017. 22(12): p. 1004-1010.

Walther, D.M., et al., Widespread Proteome Remodeling and Aggregation in Aging C. elegans. Cell, 2015. 161(4): p. 919-32.

Morley, J.F., et al., The threshold for polyglutamine-expansion protein aggregation and cellular toxicity is dynamic and influenced by aging in Caenorhabditis elegans. Proc Natl Acad Sci U S A, 2002. 99(16): p. 10417-22.

Hartl, F.U., Heat shock proteins in protein folding and membrane translocation. Semin Immunol, 1991. 3(1): p. 5-16. 
202.

203.

204.

205.

206.

207.

208.

209.

210.

211.

212.

213.

214.

215.

Link, C.D., et al., Direct observation of stress response in Caenorhabditis elegans using a reporter transgene. Cell Stress Chaperones, 1999. 4(4): p. 235-42.

Walker, G.A., et al., Heat shock protein accumulation is upregulated in a long-lived mutant of Caenorhabditis elegans. J Gerontol A Biol Sci Med Sci, 2001. 56(7): p. B2817.

Mendenhall, A.R., et al., Expression of a single-copy hsp-16.2 reporter predicts life span. J Gerontol A Biol Sci Med Sci, 2012. 67(7): p. 726-33.

Shigenaga, M.K., T.M. Hagen, and B.N. Ames, Oxidative damage and mitochondrial decay in aging. Proc Natl Acad Sci U S A, 1994. 91(23): p. 10771-8.

Boengler, K., et al., Mitochondria and ageing: role in heart, skeletal muscle and adipose tissue. J Cachexia Sarcopenia Muscle, 2017. 8(3): p. 349-369.

Regmi, S.G., S.G. Rolland, and B. Conradt, Age-dependent changes in mitochondrial morphology and volume are not predictors of lifespan. Aging (Albany NY), 2014. 6(2): p. 118-30.

Boveris, A. and B. Chance, The mitochondrial generation of hydrogen peroxide. General properties and effect of hyperbaric oxygen. Biochem J, 1973. 134(3): p. 707-16.

Goudeau, J. and H. Aguilaniu, Carbonylated proteins are eliminated during reproduction in C. elegans. Aging Cell, 2010. 9(6): p. 991-1003.

Ristow, M. and K. Zarse, How increased oxidative stress promotes longevity and metabolic health: The concept of mitochondrial hormesis (mitohormesis). Exp Gerontol, 2010. 45(6): p. 410-8.

Ristow, M., et al., Antioxidants prevent health-promoting effects of physical exercise in humans. Proc Natl Acad Sci U S A, 2009. 106(21): p. 8665-70.

Safdar, A., et al., Aberrant mitochondrial homeostasis in the skeletal muscle of sedentary older adults. PLoS One, 2010. 5(5): p. e10778.

Go, Y.M. and D.P. Jones, Redox theory of aging: implications for health and disease. Clin Sci (Lond), 2017. 131(14): p. 1669-1688.

Zevian, S.C. and J.L. Yanowitz, Methodological considerations for heat shock of the nematode Caenorhabditis elegans. Methods, 2014. 68(3): p. 450-7.

Davis, J.R., Y. Li, and C.H. Rankin, Effects of developmental exposure to ethanol on Caenorhabditis elegans. Alcohol Clin Exp Res, 2008. 32(5): p. 853-67. 


\section{Supplemental material}

\section{Figure S 1}

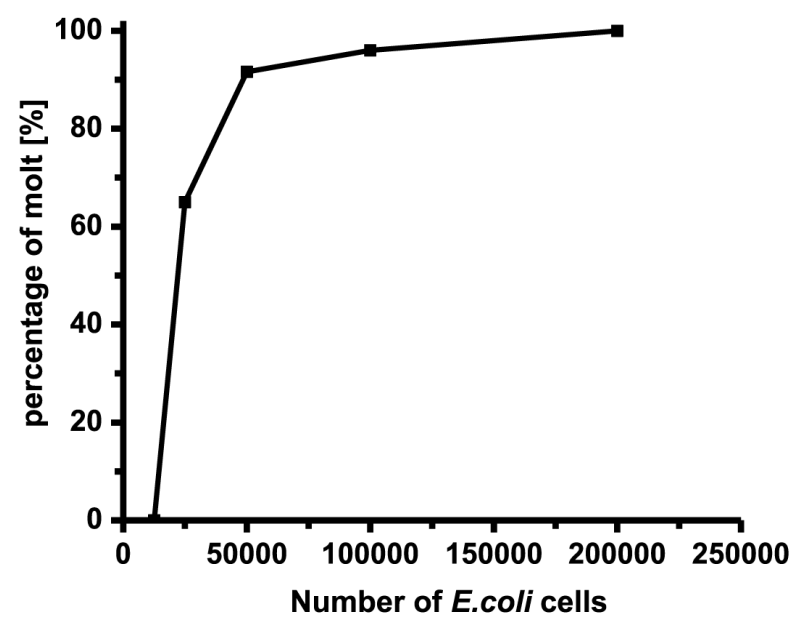

Figure S 1 Food is required for L1 larvae for development.

Single L1 larvae that hatch after 1 day was fed with OP50 (E. coli) in each group with different concertation of bacteria for 4 days. Number of $E$. coli cells were calculated before the assay with using $O D$ 600.1.25*10 $0^{4}$ E. coli cells per worm: $0 \%$ of L1 larvae developed to L2 stage, $\mathrm{n}=20.2 .5^{*} 10^{4}$ E. coli cells per worm: $65 \%$ of L1 larvae developed to L2 stage, $\mathrm{n}=23.5^{*} 10^{4} E$. coli cells per worm: $91.6 \%$ of L1 larvae developed to L2 stage, $\mathrm{n}=24$. $1 * 10^{5}$ E. coli cells per worm: $96 \%$ of L1 larvae developed to L2 stage, $\mathrm{n}=25.2 * 10^{5}$ E. coli cells per worm: $100 \%$ of L1 larvae developed to L2 stage, $\mathrm{n}=36$. Percentage of molt in each group was shown as single dot in figure. 


\section{Figure S 2}

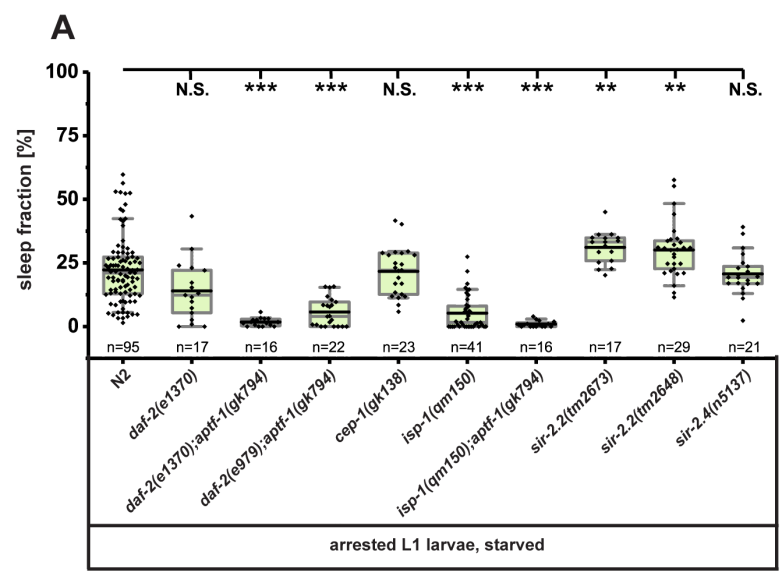

B

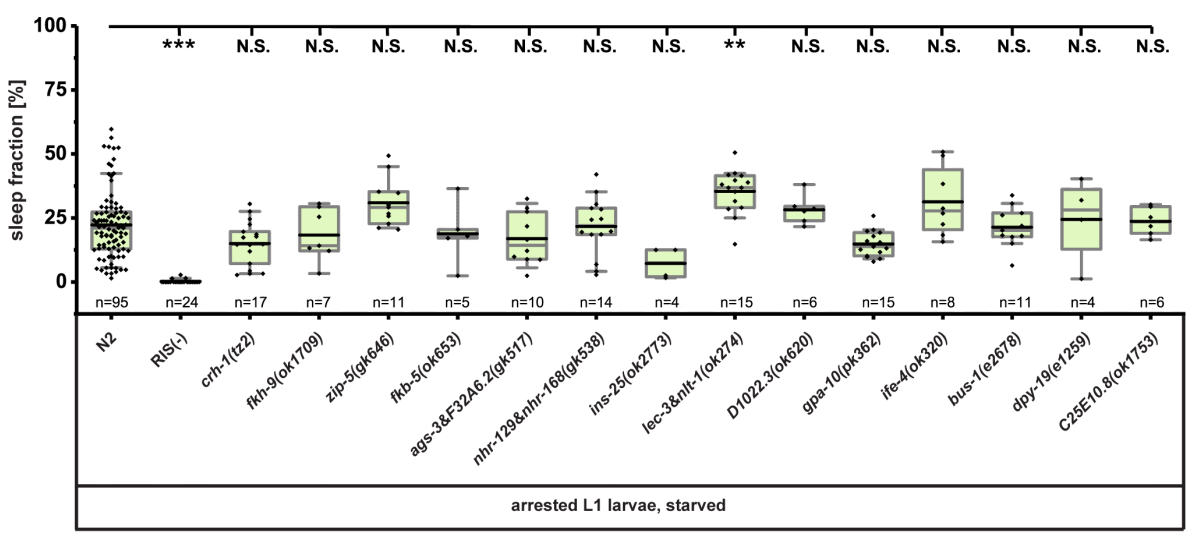

C

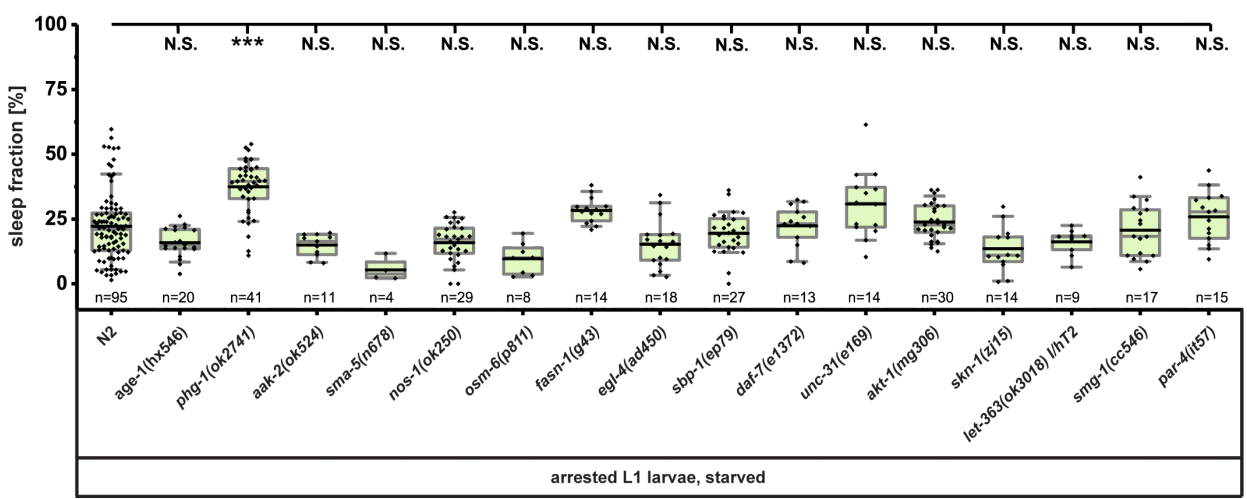

Figure S 2 Screen of mutation for sleep alteration in arrested L1 larvae during starvation.

(A) Sleep fraction of arrested L1 larvae. Individual worm was shown in the box plot as dots, significant median of sleep fraction: N2 (control): 21.1\%; daf-2(e1370)/aptf-1(gk794): 1.6\%, $* * * \mathrm{p}<0.001$; daf-2(e979)/aptf-1(gk794): 4\%, ***p<0.001; isp-1(qm150): 1.6\%, ***, <0.001; isp1(qm150)/aptf-1(gk794): $0.7 \%, * * * \mathrm{p}<0.001 ; \operatorname{sir}-2.2(\operatorname{tm} 2673): 33.2 \%,{ }^{*} \mathrm{p}=0.011 ; \operatorname{sir}-2.2(\operatorname{tm} 2648)$ : $30.3 \%, * p=0.017$. (B) Sleep fraction of arrested L1 larvae. Individual worm was shown in the box plot as dots, significant median of sleep fraction: N2 (control): $21.1 \%$; RIS ablated worms RIS(-): $0 \%, * * * \mathrm{p}<0.001$; lec-3\&nlt-1(ok274): 36.8\%, ***p<0.001. (C) Sleep fraction of arrested L1 larvae. Individual worm was shown in the box plot as dots, significant median of sleep fraction: N2 (control): $21.1 \%$; phg-1(ok2741): $39.6 \%, * * * p<0.001$. Details of sleep fraction of all the mutants 
are shown in table S6. Mann-Whitney U test was used to calculate the p-value and BenjaminiHochberg Procedure was used to confirm the significance by multiple comparisons.

Figure S 3

DAF-18 rescue of arrested L1 larvae, starved

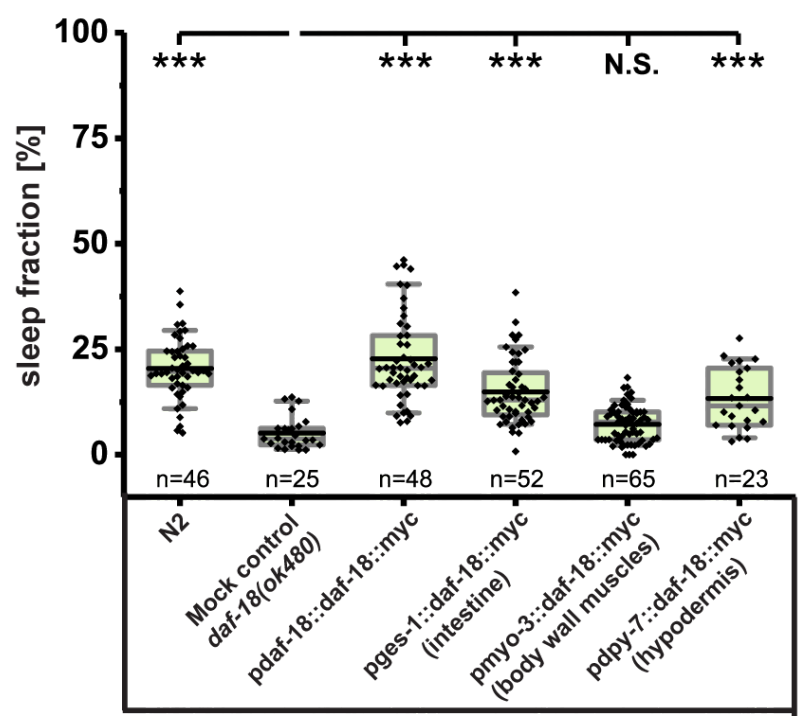

Figure S 3 DAF-18 rescue across different tissues of arrested L1 larvae during starvation.

The extrachromosomal transgenes of daf-18 were used in the rescue assay. DAF-18 was expressed from the endogenous promoter in the whole worm; in the intestine (pges-1); in the body wall muscles (pmyo-3) and the hypodermis (pdpy-7). Rescue strains carry a mutation of daf-18(ok480) and an extrachromosomal fluorescence (pstr-1::GFP) marker. A mock control was used for comparison, which only expresses the fluorescence (pstr$1: \because G F P)$ marker in daf-18(ok480) mutation background. Increased sleep fractions were shown in the strain of the whole worm-rescue (median of sleep fraction): $20.5 \%, * * * p<0.001$; the intestinerescue: $12.9 \%$. $* * * \mathrm{p}<0.001$ and the hypodermis rescue: $11.6 \%, * * * \mathrm{p}<0.001$. Individual worm was shown in the box plot as dots. Details of sleep fraction of DAF-18 rescue are shown in Table S6. Mann-Whitney U test was used to calculate the $\mathrm{p}$ value, and Benjamini-Hochberg Procedure was used to confirm the significance by multiple comparisons.

Figure S 4
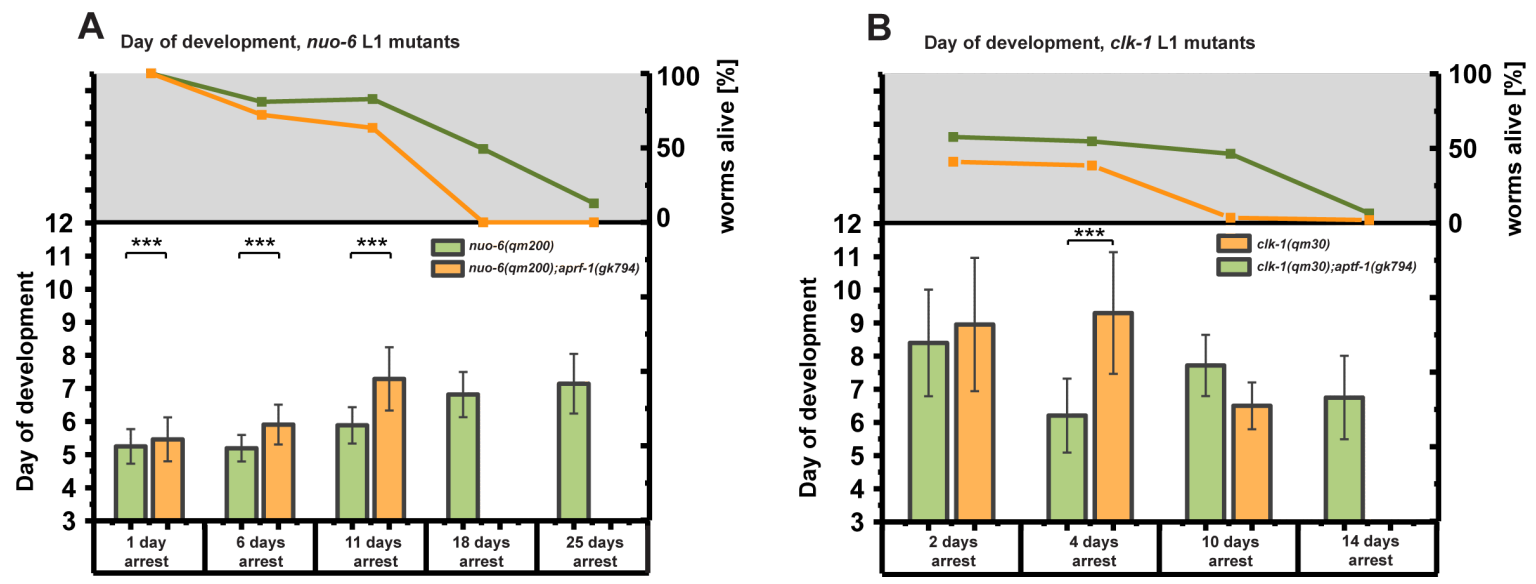

Figure S 4 Lack of sleep increases the development period of mutant larvae nuo-6(qm200) and clk1 (qm30) by re-entering the development after starvation.

(A) Upper part: percentage of the arrested L1 larvae that are able to re-enter development after starvation for $1,6,11,18,25$ days. Green line: nuo-6(qm200), $\mathrm{n}>60$; orange line: nuo-6(qm200)/aptf-1(gk794), $\mathrm{n}>60$. Lower part: days, which worms used to develop into the late-L4 stage from L1 larvae. L1 larval nuo-6(qm200)/aptf-1(gk794) double mutants after 1, 6, 11 days starvation showed a significant increase of developmental time compared to the nuo-6(qm200) mutants, $* * * p<0.001$. (B) Upper part: percentage of arrested L1 larvae that are able to re-enter development after starvation for 2, 4, 10, 14 days. Green line: clk-1(qm30), $\mathrm{n}>60$; orange line: clk-1(qm30)/aptf-1(gk794), $\mathrm{n}>60$. Lower part: days, which worms used to develop into the late-L4 stage from L1 larvae. L1 larval clk-1(qm30)/aptf-1(gk794) double mutants after 4 days starvation showed a significant increase of developmental time compared to the $c l k$ 1(qm30) single mutants, $* * * \mathrm{p}<0.001$. Mann-Whitney U test. 
Table S 1

\begin{tabular}{|c|c|c|c|c|c|c|c|}
\hline \multicolumn{8}{|c|}{ Lifespan of adult under different conditions $\left(20^{\circ} \mathrm{C}\right)$} \\
\hline Strain & $\begin{array}{l}\text { Experiment } \\
\text { batch } \\
\text { (in Figure) }\end{array}$ & Treated with & $\begin{array}{c}\text { \# scored/ } \\
\text { \# total }\end{array}$ & $\begin{array}{c}\text { Mean } \\
\text { of } \\
\text { lifespan } \\
\text { (days) }\end{array}$ & $\begin{array}{l}\text { Compared } \\
\text { to }\end{array}$ & $\begin{array}{c}\Delta \\
\text { Mean } \\
\text { of } \\
\text { lifespan } \\
\end{array}$ & $\begin{array}{c}p \text { value } \\
\text { (log-rank } \\
\text { test) }\end{array}$ \\
\hline \multicolumn{8}{|c|}{ Figure 1} \\
\hline $\mathrm{N} 2$ & (A) Batch 1 & $\begin{array}{l}\text { Incubated in } \\
\text { M9 buffer }\end{array}$ & $75 / 150$ & 16.05 & & & \\
\hline aptf-1 (gk794) & (A) Batch 1 & $\begin{array}{l}\text { Incubated in } \\
\text { M9 buffer }\end{array}$ & $124 / 150$ & 15.98 & $\begin{array}{l}\text { N2 (A) } \\
\text { Batch } 1\end{array}$ & & $\begin{array}{l}0.77 \\
\text { N.S. }\end{array}$ \\
\hline N2 & (A) Batch 2 & $\begin{array}{l}\text { Incubated in } \\
\text { M9 buffer }\end{array}$ & $257 / 309$ & 16.74 & & & \\
\hline aptf-1 (gk794) & (A) Batch 2 & $\begin{array}{l}\text { Incubated in } \\
\text { M9 buffer }\end{array}$ & $255 / 301$ & 17.15 & $\begin{array}{l}\text { N2 (A) } \\
\text { Batch } 2\end{array}$ & & $\begin{array}{l}0.22, \\
\text { N.S. }\end{array}$ \\
\hline RIS(-) & (A) Batch 2 & $\begin{array}{l}\text { Incubated in } \\
\text { M9 buffer }\end{array}$ & $271 / 308$ & 13.66 & $\begin{array}{l}\text { N2 (A) } \\
\text { Batch } 2\end{array}$ & $-18.4 \%$ & $\begin{array}{c}<0.001 \\
\quad * * *\end{array}$ \\
\hline N2 & (B) Batch 1 & $\begin{array}{l}\text { M9 buffer } \\
\text { starved }\end{array}$ & $78 / 185$ & 30.82 & & & \\
\hline aptf-1 (gk794) & (B) Batch 1 & $\begin{array}{l}\text { M9 buffer } \\
\text { starved }\end{array}$ & $43 / 111$ & 30.14 & $\begin{array}{l}\text { N2 (B) } \\
\text { Batch } 1\end{array}$ & & $\begin{array}{l}0.36 \\
\text { N.S. }\end{array}$ \\
\hline N2 & (B) Batch 2 & $\begin{array}{l}\text { M9 buffer } \\
\text { starved }\end{array}$ & $180 / 307$ & 34.35 & & & \\
\hline aptf-1 (gk794) & (B) Batch 2 & $\begin{array}{l}\text { M9 buffer } \\
\text { starved }\end{array}$ & $155 / 295$ & 35.95 & $\begin{array}{l}\text { N2 (B) } \\
\text { Batch } 2\end{array}$ & & $\begin{array}{l}0.23 \\
\text { N.S. }\end{array}$ \\
\hline$R I S(-)$ & (B) Batch 2 & $\begin{array}{l}\text { M9 buffer } \\
\text { starved }\end{array}$ & $134 / 300$ & 34.62 & $\begin{array}{l}\text { N2 (B) } \\
\text { Batch } 2\end{array}$ & & $\begin{array}{l}0.83 \\
\text { N.S. }\end{array}$ \\
\hline \multicolumn{8}{|c|}{ Lifespan on NGM plate $\left(20^{\circ} \mathrm{C}\right)$} \\
\hline N2 & Control & Feeding & $39 / 50$ & 19.75 & & & \\
\hline $\operatorname{aptf-1}(g k 794)$ & Control & Feeding & $42 / 50$ & 17.83 & & & \\
\hline N2 & (C) & $\begin{array}{l}\text { Intermittent } \\
\text { Fasting }\end{array}$ & $73 / 100$ & 23.69 & & & \\
\hline aptf-1(gk794) & (C) & $\begin{array}{l}\text { Intermittent } \\
\text { Fasting }\end{array}$ & $97 / 105$ & 22.71 & $\mathrm{~N} 2(\mathrm{C})$ & & 0.1 , N.S. \\
\hline $\mathrm{N} 2$ & (D) & $\begin{array}{l}10 \text { mM 2- } \\
\text { deoxy-D- } \\
\text { glucose }\end{array}$ & $89 / 100$ & 22.84 & N2 Control & $+15.7 \%$ & $\begin{array}{c}<0.001 \\
* * *\end{array}$ \\
\hline aptf-1 $(g k 794)$ & (D) & $\begin{array}{l}10 \mathrm{mM} 2- \\
\text { deoxy-D- } \\
\text { glucose }\end{array}$ & $84 / 100$ & 24.35 & N2 (D) & $+6.6 \%$ & $\begin{array}{l}0.029 \\
\quad *\end{array}$ \\
\hline $\mathrm{N} 2$ & (E) & $\begin{array}{l}100 \text { mM D- } \\
\text { Glucose }\end{array}$ & $55 / 100$ & 19.25 & N2 Control & & $\begin{array}{l}0.52 \\
\text { N.S. }\end{array}$ \\
\hline aptf-1 $(g k 794)$ & (E) & $\begin{array}{l}100 \mathrm{mM} \text { D- } \\
\text { Glucose }\end{array}$ & $58 / 102$ & 19.48 & N2 (E) & & $\begin{array}{l}0.95 \\
\text { N.S. }\end{array}$ \\
\hline
\end{tabular}




\begin{tabular}{|c|c|c|c|c|c|c|c|}
\hline $\mathrm{N} 2$ & (F) & $\begin{array}{c}0.1 \mathrm{mM} \\
\text { Sodium Azide }\end{array}$ & $113 / 120$ & 17.84 & N2 Control & $-9.7 \%$ & $\begin{array}{l}<0.001, \\
* * *\end{array}$ \\
\hline aptf-1 (gk794) & (F) & $\begin{array}{c}0.1 \mathrm{mM} \\
\text { Sodium Azide }\end{array}$ & $98 / 116$ & 17.94 & N2 (F) & & $\begin{array}{l}0.63, \\
\text { N.S. }\end{array}$ \\
\hline $\mathrm{N} 2$ & (G) & $\begin{array}{l}\text { GFP RNAi } \\
\text { (Control) }\end{array}$ & $83 / 110$ & 14.84 & & & \\
\hline aptf-1 (gk794) & (G) & $\begin{array}{c}\text { GFP RNAi } \\
\text { (Control) }\end{array}$ & $80 / 89$ & 11.26 & $\begin{array}{l}\mathrm{N} 2(\mathrm{G}) \\
\text { Control }\end{array}$ & $-24.1 \%$ & $\begin{array}{l}<0.001, \\
* * *\end{array}$ \\
\hline $\mathrm{N} 2$ & (G) & let-363 RNAi & $90 / 107$ & 13.94 & $\begin{array}{l}\mathrm{N} 2(\mathrm{G}) \\
\text { Control }\end{array}$ & & $\begin{array}{l}0.3 \text {, } \\
\text { N.S. }\end{array}$ \\
\hline aptf-1 (gk794) & (G) & let-363 RNAi & $99 / 109$ & 12.13 & $\begin{array}{l}\text { N2 (G) let- } \\
363 \text { RNAi }\end{array}$ & $-12.9 \%$ & $\begin{array}{l}<0.01 \\
*\end{array}$ \\
\hline $\mathrm{N} 2$ & (H), (I) & $\begin{array}{l}\text { GFP RNAi } \\
\text { (Control) }\end{array}$ & $45 / 67$ & 12.09 & & & \\
\hline aptf-1 (gk794) & (H), (I) & $\begin{array}{l}\text { GFP RNAi } \\
\text { (Control) }\end{array}$ & $54 / 59$ & 11.41 & $\begin{array}{c}\mathrm{N} 2(\mathrm{H}),(\mathrm{I}) \\
\text { Control }\end{array}$ & & $\begin{array}{l}0.18, \\
\text { N.S. }\end{array}$ \\
\hline $\mathrm{N} 2$ & $(\mathrm{H})$ & pha-4 RNAi & $76 / 81$ & 10.25 & $\begin{array}{c}\text { N2 }(\mathrm{H}),(\mathrm{I}) \\
\text { Control }\end{array}$ & $-15.3 \%$ & $\begin{array}{l}<0.001, \\
* * *\end{array}$ \\
\hline aptf-1 (gk794) & (H) & pha-4 RNAi & $73 / 80$ & 11.12 & $\begin{array}{l}\text { N2 (H) } \\
\text { pha-4 } \\
\text { RNAi }\end{array}$ & & $\begin{array}{l}0.09 \text {, } \\
\text { N.S. }\end{array}$ \\
\hline $\mathrm{N} 2$ & (I) & $s k n-1$ RNAi & $64 / 74$ & 10.99 & $\begin{array}{c}\mathrm{N} 2(\mathrm{H}),(\mathrm{I}) \\
\text { Control }\end{array}$ & $-9.1 \%$ & $0.02, *$ \\
\hline aptf-1 (gk794) & (I) & $s k n-1$ RNAi & $83 / 86$ & 11.72 & $\begin{array}{c}\mathrm{N} 2 \text { (I) } s k n- \\
1 \text { RNAi }\end{array}$ & & 0.2, N.S. \\
\hline $\mathrm{N} 2$ & $(\mathrm{~J}),(\mathrm{K})$ & $\begin{array}{l}\text { Feeding } \\
\text { Control }\end{array}$ & $39 / 50$ & 19.75 & & & \\
\hline aptf-1 (gk794) & $(\mathrm{J}),(\mathrm{K})$ & $\begin{array}{l}\text { Feeding } \\
\text { Control }\end{array}$ & $42 / 50$ & 17.83 & & & \\
\hline daf-2(e1370) & (J) Batch 1 & Feeding & $35 / 50$ & 44.29 & & & \\
\hline $\begin{array}{c}\text { daf- } \\
\text { 2(e1370);aptf- } \\
\text { 1(gk794) }\end{array}$ & (J) Batch 1 & Feeding & $33 / 50$ & 43.37 & $\begin{array}{c}d a f-2 \\
(e 1370)(\mathrm{J}) \\
\text { Batch } 1 \\
\end{array}$ & & $\begin{array}{l}0.57 \text {, } \\
\text { N.S. }\end{array}$ \\
\hline$d a f-2(e 1370)$ & (J) Batch 2 & Feeding & $26 / 50$ & 38.70 & & & \\
\hline $\begin{array}{c}d a f- \\
\text { 2(el370);aptf- } \\
\text { 1(gk794) }\end{array}$ & (J) Batch 2 & Feeding & $32 / 50$ & 37.82 & $\begin{array}{c}d a f-2 \\
\text { (el370) (J) } \\
\text { Batch 2 } \\
\end{array}$ & & $\begin{array}{l}0.59 \\
\text { N.S. }\end{array}$ \\
\hline$d a f-16(m g D f 50)$ & (K) Batch 1 & Feeding & $52 / 58$ & 13.12 & & & \\
\hline $\begin{array}{c}d a f- \\
16(m g D f 50), a p t f \\
-1(g k 794)\end{array}$ & (K) Batch 1 & Feeding & $45 / 58$ & 13.18 & $\begin{array}{c}\text { daf-16 } \\
\text { (mgDf50) } \\
\text { (K) Btach1 }\end{array}$ & & $\begin{array}{l}0.92 \\
\text { N.S. }\end{array}$ \\
\hline$d a f-16(m g D f 50)$ & (K) Batch 2 & Feeding & $102 / 120$ & 16.07 & & & \\
\hline $\begin{array}{c}d a f- \\
16(m g D f 50), a p t f \\
-1(g k 794)\end{array}$ & (K) Batch 2 & Feeding & $95 / 120$ & 14.82 & $\begin{array}{c}d a f-16 \\
\text { (mgDf50) } \\
\text { (K) Btach2 }\end{array}$ & $-7.78 \%$ & $\begin{array}{l}<0.001, \\
* * *\end{array}$ \\
\hline
\end{tabular}




\begin{tabular}{|c|c|c|c|c|c|c|c|}
\hline$d a f-16(m g D f 50)$ & (K) Batch 3 & Feeding & $49 / 60$ & 15.73 & & & \\
\hline $\begin{array}{c}d a f- \\
16(m g D f 50), a p t f \\
-1(g k 794)\end{array}$ & (K) Batch 3 & Feeding & $48 / 60$ & 15.02 & $\begin{array}{c}d a f-16 \\
\text { (mgDff50) } \\
\text { (K) Btach3 }\end{array}$ & $-4.51 \%$ & $\begin{array}{c}0.015, \\
*\end{array}$ \\
\hline $\mathrm{N} 2$ & (L) & $\begin{array}{l}\text { Starved } \\
\text { Control }\end{array}$ & $60 / 100$ & 29.81 & & & \\
\hline aptf-1 $(g k 794)$ & (L) & $\begin{array}{l}\text { Starved } \\
\text { Control }\end{array}$ & $63 / 100$ & 29.46 & $\begin{array}{l}\text { N2 (L) } \\
\text { Control }\end{array}$ & & $\begin{array}{l}0.65 \\
\text { N.S. }\end{array}$ \\
\hline daf-16(mgDf50) & (L) & Starved & $48 / 60$ & 21.19 & & & \\
\hline $\begin{array}{c}d a f- \\
16(m g D f 50), a p t f \\
-1(g k 794)\end{array}$ & (L) & Starved & $52 / 62$ & 20.99 & $\begin{array}{c}d a f-16 \\
(m g D f 50) \\
(\mathrm{L})\end{array}$ & & $\begin{array}{l}0.93 \\
\text { N.S. }\end{array}$ \\
\hline
\end{tabular}

Table S 1 Detail of adult lifespans with replicates are shown. Table is related to figure 1. Displayed are the strains used, the related figure sub number with replicated batches, the experimental treatments, the scored animals of the total animals number (the missing worms were censored), the mean lifespan is estimated from the log-rank test, against which group was tested, the difference with the compared group and the $p$ value of the log-rank test.

Table S 2

Survival span of arrested L1 larvae under different conditions, starved $\left(20^{\circ} \mathrm{C}\right)$

\begin{tabular}{|c|c|c|c|c|c|c|c|}
\hline Strain & $\begin{array}{c}\text { Experiment } \\
\text { (in Figure) }\end{array}$ & Treated with & $\begin{array}{c}\text { min. } \\
\text { counting }\end{array}$ & $\begin{array}{c}50 \% \\
\text { alive at } \\
\text { (days) }\end{array}$ & $\begin{array}{c}\text { Compare } \\
\mathrm{d} \text { to }\end{array}$ & $\begin{array}{c}\Delta \\
50 \% \\
\text { alive }\end{array}$ & $\begin{array}{c}p \text { value } \\
\text { in } 50 \% \\
\text { alive } \\
\text { (Fisher's } \\
\text { exact } \\
\text { test) }\end{array}$ \\
\hline
\end{tabular}

Figure 2

\begin{tabular}{|c|c|c|c|c|c|c|c|}
\hline $\mathrm{N} 2$ & (A) & $\begin{array}{c}\text { M9 } \\
\text { Control }\end{array}$ & $>50$ & 23.45 & & & \\
\hline aptf-1 (gk794) & (A) & $\begin{array}{c}\text { M9 } \\
\text { Control }\end{array}$ & $>50$ & 11.61 & & & \\
\hline$f l p-11(\operatorname{tm} 2706)$ & (A) & M9 & $>50$ & 21.87 & $\begin{array}{l}\mathrm{N} 2(\mathrm{~A}) \\
\text { Control }\end{array}$ & $-6.74 \%$ & $\begin{array}{l}0.006, * * \\
\text { in day } 21\end{array}$ \\
\hline N2 & (B) & NGM & $>100$ & 30.75 & & & \\
\hline aptf-1 (gk794) & (B) & NGM & $>100$ & 23.97 & N2 (B) & $-22.1 \%$ & $\begin{array}{l}<0.001, \\
* * * \text { in } \\
\text { day } 23\end{array}$ \\
\hline N2 & (C) & M9 Control & $>50$ & 22.89 & & & \\
\hline aptf-1 $(g k 794)$ & (C) & M9 Control & $>50$ & 10.62 & & & \\
\hline $\mathrm{N} 2$ & (C) & $\begin{array}{c}1 \mathrm{mM} \\
\text { Cycloheximide }\end{array}$ & $>50$ & 10.22 & $\begin{array}{l}\mathrm{N} 2(\mathrm{C}) \\
\text { Control }\end{array}$ & $-55.4 \%$ & $\begin{array}{l}<0.001, \\
* * * \text { in } \\
\text { day } 10 \\
\end{array}$ \\
\hline aptf-1 (gk794) & (C) & $\begin{array}{c}1 \mathrm{mM} \\
\text { Cycloheximide }\end{array}$ & $>50$ & 7.49 & $\begin{array}{c}\text { aptf-1 } \\
\text { (C) } \\
\text { Control }\end{array}$ & $-29.5 \%$ & $\begin{array}{c}<0.001, \\
* * * \text { in } \\
\text { day } 7 \\
\end{array}$ \\
\hline
\end{tabular}




\begin{tabular}{|c|c|c|c|c|c|c|c|}
\hline $\mathrm{N} 2$ & (C) & $\begin{array}{c}2 \mathrm{mM} \\
\text { Cycloheximide }\end{array}$ & $>50$ & 8.1 & $\begin{array}{l}\mathrm{N} 2(\mathrm{C}) \\
\text { Control }\end{array}$ & $-64.6 \%$ & $\begin{array}{c}<0.001, \\
* * * \text { in } \\
\text { day } 8\end{array}$ \\
\hline aptf-1 (gk794) & (C) & $\begin{array}{c}2 \mathrm{mM} \\
\text { Cycloheximide }\end{array}$ & $>50$ & 7.12 & $\begin{array}{c}\text { aptf-1 } \\
\text { (C) } \\
\text { Control }\end{array}$ & $-32.9 \%$ & $\begin{array}{c}<0.001 \\
* * * \text { in } \\
\text { day } 7\end{array}$ \\
\hline $\mathrm{N} 2$ & (D) & M9 Control & $>60$ & 17.97 & & & \\
\hline aptf-1 (gk794) & (D) & M9 Control & $>60$ & 9.54 & & & \\
\hline $\mathrm{N} 2$ & (D) & $\begin{array}{c}0.15 \mathrm{mM} \\
\text { Methionine }\end{array}$ & $>60$ & 17.20 & $\begin{array}{l}\mathrm{N} 2(\mathrm{D}) \\
\text { Control }\end{array}$ & $-4.3 \%$ & N.S. \\
\hline aptf-1 $(g k 794)$ & (D) & $\begin{array}{c}0.15 \mathrm{mM} \\
\text { Methionine }\end{array}$ & $>60$ & 10.06 & $\begin{array}{c}\text { aptf-1 } \\
\text { (D) } \\
\text { Control }\end{array}$ & $+5.5 \%$ & $\begin{array}{c}0.0069 \\
* * \text { in } 9 \\
\text { day }\end{array}$ \\
\hline $\mathrm{N} 2$ & (D) & $\begin{array}{c}1 \mathrm{mM} \\
\text { Methionine }\end{array}$ & $>60$ & 15.80 & $\begin{array}{l}\mathrm{N} 2(\mathrm{D}) \\
\text { Control }\end{array}$ & $-12.1 \%$ & $\begin{array}{l}0.013, * \\
\text { in day } 15\end{array}$ \\
\hline aptf-1 (gk794) & (D) & $\begin{array}{l}1 \mathrm{mM} \\
\text { Methionine }\end{array}$ & $>60$ & 9.90 & $\begin{array}{c}\text { aptf-1 } \\
\text { (D) } \\
\text { Control }\end{array}$ & $+3.8 \%$ & $\begin{array}{l}0.015, * \\
\text { in } 9 \text { day }\end{array}$ \\
\hline $\mathrm{N} 2$ & (E) & $\begin{array}{c}\text { M9 } \\
\text { Control }\end{array}$ & $>60$ & 26.9 & & & \\
\hline aptf-1 $(g k 794)$ & (E) & M9 Control & $>60$ & 10.05 & & & \\
\hline N2 & (E) & $\begin{array}{c}1 \mu \mathrm{M} \text { Sodium } \\
\text { azide }\end{array}$ & $>60$ & 20.8 & $\begin{array}{l}\mathrm{N} 2(\mathrm{E}) \\
\text { Control }\end{array}$ & $-22.7 \%$ & $\begin{array}{l}<0.001, \\
* * * \text { in } \\
\text { day } 21\end{array}$ \\
\hline aptf-1 $(g k 794)$ & (E) & $\begin{array}{c}1 \mu \mathrm{M} \text { Sodium } \\
\text { azide }\end{array}$ & $>60$ & 10.6 & $\begin{array}{c}\text { aptf-1 } \\
\text { (E) } \\
\text { Control }\end{array}$ & & $\begin{array}{l}\text { N.S. in } \\
\text { day } 10\end{array}$ \\
\hline \multicolumn{8}{|c|}{ Figure 3} \\
\hline $\mathrm{N} 2$ & $\begin{array}{l}\text { (A) } \\
\text { (B) Batch } 1\end{array}$ & $\begin{array}{l}0.1 \% \text { DMSO, } \\
0.1 \% \text { Ethanol }\end{array}$ & $>60$ & 26.63 & & & \\
\hline aptf-1 $(g k 794)$ & $\begin{array}{l}\text { (A) } \\
\text { (B) Batch } 1\end{array}$ & $\begin{array}{l}0.1 \% \text { DMSO, } \\
0.1 \% \text { Ethanol }\end{array}$ & $>60$ & 24.30 & & $+115 \%$ & $\begin{array}{c}<0.001, \\
* * * \text { in } \\
\text { day } 11\end{array}$ \\
\hline $\mathrm{N} 2$ & (A) & $\begin{array}{c}\text { 0.1\% DMSO, } \\
0.1 \% \text { Ethanol, } \\
50 \mu \mathrm{M} \\
\text { Rapamycin }\end{array}$ & $>60$ & 20.60 & N2 (A) & $-22.6 \%$ & $\begin{array}{l}<0.001, \\
* * * \text { in } \\
\text { day } 21\end{array}$ \\
\hline aptf-1 (gk794) & (A) & $\begin{array}{c}0.1 \% \text { DMSO, } \\
0.1 \% \text { Ethanol, } \\
50 \mu \mathrm{M} \\
\text { Rapamycin }\end{array}$ & $>60$ & 22.29 & $\begin{array}{l}\text { aptf-1 } \\
\text { (A) }\end{array}$ & $-8.2 \%$ & $\begin{array}{c}<0.01, \\
* * \text { in day } \\
25\end{array}$ \\
\hline $\mathrm{N} 2$ & (A) Batch 1 & $\begin{array}{c}0.1 \% \text { DMSO, } \\
0.1 \% \text { Ethanol, } \\
100 \mu \mathrm{M} \\
\text { Rapamycin }\end{array}$ & $>60$ & 20.36 & N2 (A) & $-23.4 \%$ & $\begin{array}{l}<0.001, \\
* * * \text { in } \\
\text { day } 21\end{array}$ \\
\hline aptf-1 (gk794) & (A) Batch 1 & $\begin{array}{c}0.1 \% \text { DMSO, } \\
0.1 \% \text { Ethanol, } \\
100 \mu \mathrm{M} \\
\text { Rapamycin }\end{array}$ & $>60$ & 21.00 & $\begin{array}{l}\text { aptf-1 } \\
\text { (A) }\end{array}$ & $-13.6 \%$ & $\begin{array}{l}<0.001, \\
* * * \text { in } \\
\text { day } 25\end{array}$ \\
\hline
\end{tabular}




\begin{tabular}{|c|c|c|c|c|c|c|c|}
\hline $\mathrm{N} 2$ & (A) Batch 2 & $\begin{array}{c}0.1 \% \text { DMSO, } \\
0.1 \% \text { Ethanol, } \\
100 \mu \mathrm{M} \\
\text { Rapamycin }\end{array}$ & $>60$ & 22.33 & $\begin{array}{l}\text { N2 (B) } \\
\text { Batch } 2\end{array}$ & $-11.2 \%$ & $\begin{array}{l}<0.001 \\
* * * \text { in } \\
\text { day } 23\end{array}$ \\
\hline $\operatorname{aptf-1}(g k 794)$ & (A) Batch 2 & $\begin{array}{c}0.1 \% \text { DMSO, } \\
0.1 \% \text { Ethanol, } \\
100 \mu \mathrm{M} \\
\text { Rapamycin }\end{array}$ & $>60$ & 20.62 & $\begin{array}{l}\text { N2 (B) } \\
\text { Batch } 2\end{array}$ & $-13.1 \%$ & $\begin{array}{c}<0.001 \\
* * * \text { in } \\
\text { day } 23\end{array}$ \\
\hline N2 & (B), (C) & M9 Control & $>60$ & 25.14 & & & \\
\hline $\operatorname{aptf-1}(g k 794)$ & (B), (C) & M9 Control & $>60$ & 11.28 & & & \\
\hline N2 & (B) Batch 1 & $\begin{array}{l}0.1 \% \text { DMSO, } \\
0.1 \% \text { Ethanol }\end{array}$ & $>60$ & 26.63 & & & \\
\hline $\operatorname{aptf-1}(g k 794)$ & (B) Batch 1 & $\begin{array}{l}0.1 \% \text { DMSO, } \\
0.1 \% \text { Ethanol }\end{array}$ & $>60$ & 24.30 & & $+115 \%$ & $\begin{array}{c}<0.001, \\
* * * \text { in } \\
\text { day } 11\end{array}$ \\
\hline $\mathrm{N} 2$ & (B) Batch 2 & $\begin{array}{l}0.1 \% \text { DMSO, } \\
0.1 \% \text { Ethanol }\end{array}$ & $>60$ & 25.15 & & & \\
\hline aptf-1 (gk794) & (B) Batch 2 & $\begin{array}{l}0.1 \% \text { DMSO, } \\
0.1 \% \text { Ethanol }\end{array}$ & $>60$ & 23.72 & & $+110 \%$ & $\begin{array}{c}<0.001 \\
* * * \text { in } \\
\text { day } 11 \\
\end{array}$ \\
\hline N2 & (C) & $0.1 \%$ DMSO & $>60$ & 26 & & & \\
\hline aptf-1 (gk794) & (C) & $0.1 \%$ DMSO & $>60$ & 10.54 & $\begin{array}{c}\text { aptf-1(C) } \\
\text { Control }\end{array}$ & & N.S. \\
\hline N2 & (D) & $\begin{array}{c}\text { M9 } \\
\text { Control }\end{array}$ & $>60$ & 26.9 & & & \\
\hline aptf-1 (gk794) & (D) & M9 Control & $>60$ & 10.05 & & & \\
\hline N2 & (D) & $0.1 \%$ Ethanol & $>60$ & 24.5 & $\begin{array}{l}\text { N2 (D) } \\
\text { Control }\end{array}$ & $-8.9 \%$ & $\begin{array}{c}<0.001 \\
* * * \text { in } \\
\text { day } 23\end{array}$ \\
\hline aptf-1 (gk794) & (D) & $0.1 \%$ Ethanol & $>60$ & 24.4 & $\begin{array}{l}\text { aptf-1(D) } \\
\text { Control }\end{array}$ & $\begin{array}{c}+142.8 \\
\%\end{array}$ & $\begin{array}{c}<0.001, \\
* * * \text { in } \\
\text { day } 23\end{array}$ \\
\hline
\end{tabular}

Figure 4

\begin{tabular}{|c|c|c|c|c|c|c|c|}
\hline N2 & (A) & $\begin{array}{l}\text { M9 buffer at } \\
20^{\circ} \mathrm{C} \text { Control }\end{array}$ & $>50$ & 22.89 & & & \\
\hline $\begin{array}{c}\text { gpIs1 [phsp- } \\
16.2:: \mathrm{GFP} \text { ] }\end{array}$ & (A) & $\begin{array}{c}\text { M9 buffer at } \\
20^{\circ} \mathrm{C}\end{array}$ & $>60$ & 13.12 & & & \\
\hline $\begin{array}{c}\text { goeIs } 240[\mathrm{phsp} 1 \\
6.2: \text { flp- } \\
11:: \text { SL2mKate2 } \\
\text { unc-54-3UTR, } \\
\text { unc-119(+)]. }\end{array}$ & (A) & $\begin{array}{c}\text { M9 buffer at } \\
20^{\circ} \mathrm{C}\end{array}$ & $>60$ & 8.36 & $\begin{array}{c}\text { gpIs1 } \\
\text { [phsp- } \\
16- \\
2:: \mathrm{GFP}] \\
(\mathrm{A}) \\
\left(20^{\circ} \mathrm{C}\right) \\
\end{array}$ & $-36.3 \%$ & $\begin{array}{c}<0.001 \\
* * * \text { in } \\
\text { day } 9\end{array}$ \\
\hline N2 & (A) & $\begin{array}{l}\text { M9 buffer at } \\
25^{\circ} \mathrm{C} \text { Control }\end{array}$ & $>60$ & 13.63 & & & \\
\hline $\begin{array}{c}\text { gpIs1 [phsp- } \\
16.2:: \mathrm{GFP}]\end{array}$ & (A) & $\begin{array}{c}\text { M9 buffer at } \\
25^{\circ} \mathrm{C}\end{array}$ & $>60$ & 10.64 & $\begin{array}{l}\text { gpIs1 } \\
\text { [phsp- } \\
16-\end{array}$ & $-18.9 \%$ & $\begin{array}{c}<0.001 \\
* * * \text { in } \\
\text { day } 11\end{array}$ \\
\hline
\end{tabular}




\begin{tabular}{|c|c|c|c|c|c|c|c|}
\hline & & & & & $\begin{array}{c}2:: \mathrm{GFP}] \\
(\mathrm{A}) \\
\left(20^{\circ} \mathrm{C}\right)\end{array}$ & & \\
\hline $\begin{array}{c}\text { goeIs240[phsp1 } \\
\text { 6.2::flp- } \\
\text { 11::SL2mKate2 } \\
\text { unc-54-3UTR, } \\
\text { unc-119(+)]. }\end{array}$ & (A) & $\begin{array}{c}\text { M9 buffer at } \\
25^{\circ} \mathrm{C}\end{array}$ & $>60$ & 9.49 & $\begin{array}{c}\text { goeIs240 } \\
\text { [phsp16. } \\
2:: \text { flp- } \\
11:: \text { SL2 } \\
\text { mKate2u } \\
\text { nc-54- } \\
3 \text { UTR, } \\
\text { unc- } \\
119(+)]( \\
\text { A) }\left(20^{\circ} \mathrm{C}\right)\end{array}$ & & N.S. \\
\hline $\begin{array}{c}\text { gpIs1 [phsp- } \\
16.2:: \mathrm{GFP} \text { ] }\end{array}$ & (B) & $\begin{array}{c}\text { M9-Chamber } \\
\text { at } 25^{\circ} \mathrm{C}\end{array}$ & 32 & 19.88 & & & \\
\hline $\begin{array}{l}\text { goeIs240[phsp1 } \\
\text { 6.2::flp- } \\
\text { 11::SL2mKate2 } \\
\text { unc-54-3UTR, } \\
\text { unc-119(+)]. }\end{array}$ & (B) & $\begin{array}{c}\text { M9-Chamber } \\
\text { at } 25^{\circ} \mathrm{C}\end{array}$ & 40 & 19.93 & $\begin{array}{c}\text { gpIs1 } \\
\text { [phsp- } \\
16.2:: \mathrm{GF} \\
\mathrm{P}] \\
(\mathrm{K})\end{array}$ & $+0.2 \%$ & $\begin{array}{l}0.61, \\
\text { N.S. } \\
\text { (log-rank } \\
\text { test) }\end{array}$ \\
\hline $\begin{array}{l}\text { gpIs1 [phsp- } \\
16.2 \mathrm{p}:: \mathrm{GFP} \text { ] }\end{array}$ & (C) & $\begin{array}{c}\text { S Basa- } \\
\text { Chamber at } \\
25^{\circ} \mathrm{C}\end{array}$ & 19 & 17.74 & & & \\
\hline $\begin{array}{l}\text { goeIs240[phsp1 } \\
\text { 6.2::flp- } \\
\text { 11::SL2mKate2 } \\
\text { unc-54-3UTR, } \\
\text { unc-119(+)]. }\end{array}$ & (C) & $\begin{array}{c}\text { S Basa- } \\
\text { Chamber at } \\
25^{\circ} \mathrm{C}\end{array}$ & 32 & 18.13 & $\begin{array}{c}\text { gpIs1 } \\
\text { [phsp- } \\
16.2:: \mathrm{GF} \\
\mathrm{P}] \\
\text { (L) } \\
\end{array}$ & $+2 \%$ & $\begin{array}{c}0.74, \\
\text { N.S. } \\
\text { (log-rank } \\
\text { test) }\end{array}$ \\
\hline
\end{tabular}

Table S 2 Detail of survival spans of arrested L1 larvae with replicates are shown. Table is related to the figures 2-4. Displayed are the strains used, the related figure sub number with replicated batches, the experimental treatments, the scored animals number, the median survival, against which group was tested for $p$ value, the difference with the compared group and the $p$ value with the test day is estimated with the Fisher's test.

Table S 3

\begin{tabular}{|c|c|c|c|c|c|c|}
\hline \multicolumn{7}{|c|}{ Survival span of arrested L1 larvae, starved $\left(20^{\circ} \mathrm{C}\right)$} \\
\hline Strain & $\begin{array}{l}\text { Experiment } \\
\text { batch } \\
\text { (in Figure) }\end{array}$ & $\begin{array}{l}\text { min. } \\
\text { counting }\end{array}$ & $\begin{array}{c}50 \% \\
\text { alive at } \\
\text { (days) }\end{array}$ & Compared to & $\begin{array}{c}\Delta \\
50 \% \\
\text { alive }\end{array}$ & $\begin{array}{l}p \text { value in } \\
50 \% \text { alive } \\
\text { (Fisher's } \\
\text { exact test) }\end{array}$ \\
\hline \multicolumn{7}{|c|}{ Figure 5} \\
\hline $\mathrm{N} 2$ & (A) & $>50$ & 23.81 & & & \\
\hline aptf-1 $(g k 794)$ & (A) & $>50$ & 11.61 & & & \\
\hline daf-16(mgDf50) & (A) Batch 1 & $>50$ & 6.81 & N2 (A) & $-71.4 \%$ & $\begin{array}{c}<0.001, * * * \\
\text { in day } 3\end{array}$ \\
\hline $\begin{array}{c}\text { daf-16,aptf- } \\
1(g k 794)\end{array}$ & (A) Batch 1 & $>50$ & 4.72 & $\begin{array}{l}\text { daf-16(mgDf50) } \\
\text { (A) Batch } 1\end{array}$ & $-30.7 \%$ & $\begin{array}{c}<0.001, * * * \\
\text { in day } 5\end{array}$ \\
\hline$d a f-16(m g D f 50)$ & (A) Batch 2 & $>50$ & 6.77 & & & \\
\hline $\begin{array}{c}\text { daf-16,aptf- } \\
1(g k 794)\end{array}$ & (A) Batch 2 & $>50$ & 5.11 & $\begin{array}{c}d a f-16(m g D f 50) \\
\text { (A) Batch } 2\end{array}$ & $-24.5 \%$ & $\begin{array}{c}<0.001, * * * \\
\text { in day } 6\end{array}$ \\
\hline
\end{tabular}




\begin{tabular}{|c|c|c|c|c|c|c|}
\hline$d a f-16(m g D f 50)$ & (A) Batch 3 & $>50$ & 8.56 & & & \\
\hline $\begin{array}{l}\text { daf-16, aptf- } \\
\quad 1(g k 794)\end{array}$ & (A) Batch 3 & $>50$ & 5.8 & $\begin{array}{l}d a f-16(m g D f 50) \\
\text { (A) Batch } 3\end{array}$ & $-32.2 \%$ & $\begin{aligned} &<0.001, * * * \\
& \text { in day } 6\end{aligned}$ \\
\hline $\mathrm{N} 2$ & $(\mathrm{C}),(\mathrm{D}),(\mathrm{E})$ & $>60$ & 22.69 & & & \\
\hline aptf-1 $(g k 794)$ & $(\mathrm{C}),(\mathrm{D}),(\mathrm{E})$ & $>60$ & 11.71 & & & \\
\hline daf-18(ok480) & (C) Batch 1 & $>60$ & 3.28 & & & \\
\hline $\begin{array}{c}d a f-18(o k 480), \text { aptf- } \\
\text { 1(gk794) }\end{array}$ & (C) Batch 1 & $>60$ & 3.14 & $\begin{array}{l}d a f-18(o k 480) \\
\text { (C) Batch } 1\end{array}$ & & N.S. \\
\hline daf-18(ok480) & (C) Batch 2 & $>60$ & 4.16 & & & \\
\hline $\begin{array}{c}\text { daf-18(ok480), aptf- } \\
\text { 1(gk794) }\end{array}$ & (C) Batch 2 & $>60$ & 4.08 & $\begin{array}{l}\text { daf-18(ok480) } \\
\text { (C) Batch } 2\end{array}$ & & N.S. \\
\hline aak-2(ok524) & (D) & $>60$ & 7.23 & & & \\
\hline $\begin{array}{c}a a k-2 \text { (ok524), aptf- } \\
1 \text { (gk794) }\end{array}$ & (D) & $>60$ & 6.65 & $\begin{array}{l}a a k-2(o k 524) \\
\text { (D) }\end{array}$ & & N.S. \\
\hline 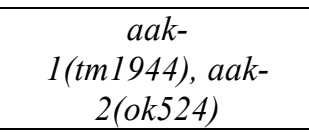 & (D) Batch 1 & $>60$ & 4.19 & & & \\
\hline $\begin{array}{c}\text { aak-1(tm1944), } \\
\text { aak-2 (ok524), aptf- } \\
1 \text { (gk794) }\end{array}$ & (D) Batch 1 & $>60$ & 5.19 & $\begin{array}{c}a a k- \\
\text { 1(tm1944), aak- } \\
\text { 2(ok524) } \\
\text { (D) Batch 1 }\end{array}$ & $+23.9 \%$ & $\begin{array}{l}0.01, * \text { in } \\
\text { day } 5\end{array}$ \\
\hline $\begin{array}{c}a a k- \\
1(\text { tm 1944), aak- } \\
\text { 2(ok524) }\end{array}$ & (D) Batch 2 & $>60$ & 3.81 & & & \\
\hline $\begin{array}{c}\text { aak-1(tm1944), } \\
\text { aak-2 (ok524), aptf- } \\
1 \text { (gk794) }\end{array}$ & (D) Batch 2 & $>60$ & 4 & $\begin{array}{c}a a k- \\
\text { 1(tm1944), aak- } \\
\text { 2(ok524) } \\
\text { (D) Batch } 2\end{array}$ & & N.S. \\
\hline sir-2.1(ok434) & (E) & $>60$ & 19.25 & & & \\
\hline $\begin{array}{l}\text { sir-2.1(ok434), } \\
\text { aptf-1(gk794) }\end{array}$ & (E) & $>60$ & 10.19 & $\begin{array}{c}\text { sir-2.1(ok434) } \\
\text { (E) }\end{array}$ & $-47.1 \%$ & $\begin{array}{l}<0.001, * * * \\
\text { in day } 11\end{array}$ \\
\hline $\mathrm{N} 2$ & (F) & $>60$ & 26.9 & & & \\
\hline $\operatorname{aptf}-1(g k 794)$ & (F) & $>60$ & 10.05 & & & \\
\hline tut-1(tm 1297) & (F) & $>60$ & 15.85 & N2 (F) & $-41.1 \%$ & $\begin{array}{c}<0.001, * * * \\
\text { in day } 19\end{array}$ \\
\hline $\begin{array}{c}\text { tut-1(tm1297), aptf- } \\
\text { 1(gk794) }\end{array}$ & (F) & $>60$ & 10.5 & $\begin{array}{l}\operatorname{aptf-1}(g k 794) \\
\text { (F) }\end{array}$ & & $\begin{array}{l}\text { N.S. in day } \\
12\end{array}$ \\
\hline
\end{tabular}

Table S 3 Details of survival spans of the arrested L1 larvae with replicates are shown. Table is related to the figure 5. Displayed are the strains used, the related figure sub number with replicated batches, the scored animals number, the median survival, against which group was tested for p-value, the difference with the compared group and the p-value with the test day is estimated with the Fisher's test 
Table S 4

\begin{tabular}{|c|c|c|c|c|c|c|}
\hline \multicolumn{7}{|c|}{ Ability to re-enter development of arrested L1 when fed $\left(20^{\circ} \mathrm{C}\right)$} \\
\hline Strain & $\begin{array}{c}\text { Experiment } \\
\text { batch } \\
\text { (in Figure) }\end{array}$ & $\begin{array}{l}\min . \\
\text { counting }\end{array}$ & $\begin{array}{l}50 \% \\
\text { alive at } \\
\text { (days) }\end{array}$ & Compared to & $\begin{array}{c}\Delta \\
50 \% \\
\text { alive }\end{array}$ & $\begin{array}{l}p \text { value in } \\
50 \% \text { alive } \\
\text { (Fisher's } \\
\text { exact test) }\end{array}$ \\
\hline \multicolumn{7}{|c|}{ Figure 5} \\
\hline N2 & (B) & $>50$ & 12.24 & & & \\
\hline daf-16(mgDf50) & (B) Batch 1 & $>50$ & 6.81 & $\begin{array}{c}\mathrm{N} 2 \\
\text { (B) Batch1 }\end{array}$ & $-44.4 \%$ & $\begin{array}{c}<0.001, * * * \\
\text { in day } 7\end{array}$ \\
\hline $\begin{array}{c}\text { daf-16,aptf- } \\
1(g k 794)\end{array}$ & (B) Batch 1 & $>50$ & 4.51 & $\begin{array}{l}d a f-16(m g D f 50) \\
\text { (B) Batch } 1\end{array}$ & $-33.8 \%$ & $\begin{array}{c}<0.001, * * * \\
\text { in day } 5\end{array}$ \\
\hline daf-16(mgDf50) & (B) Batch 2 & $>50$ & 5.06 & & & \\
\hline $\begin{array}{c}\text { daf-16,aptf- } \\
1(g k 794)\end{array}$ & (B) Batch 2 & $>50$ & 4.33 & $\begin{array}{l}d a f-16(m g D f 50) \\
\text { (B) Batch } 2\end{array}$ & $-14.4 \%$ & $\begin{aligned}< & 0.001, * * * \\
& \text { in day } 6\end{aligned}$ \\
\hline$d a f-16(m g D f 50)$ & (B) Batch 3 & $>50$ & 6.22 & & & \\
\hline $\begin{array}{c}\text { daf-16,aptf- } \\
1(g k 794)\end{array}$ & (B) Batch 3 & $>50$ & 4.35 & $\begin{array}{l}d a f-16(m g D f 50) \\
\text { (B) Batch } 3\end{array}$ & $-30.1 \%$ & $\begin{array}{c}<0.001, * * * \\
\text { in day } 5\end{array}$ \\
\hline
\end{tabular}

Table $S 4$ Detail of ability to recover from arrested L1 larvae to L4-stage with replicates are shown. Table is related to the figure 5. Displayed are the strains used, the related figure sub number with replicated batches, the scored animals number, the median recovery rate, against which group was tested for $p$ value, the difference with the compared group and the p-value with the test day is estimated with the Fisher's test.

Table S5

\begin{tabular}{|c|c|c|c|c|c|c|}
\hline \multicolumn{7}{|c|}{ Survival span of arrested L1 larvae, starved $\left(20^{\circ} \mathrm{C}\right)$} \\
\hline Strain & $\begin{array}{c}\text { Experiment } \\
\text { batch } \\
\text { (in Figure) }\end{array}$ & $\begin{array}{l}\text { min. } \\
\text { counting }\end{array}$ & $\begin{array}{c}50 \% \\
\text { alive at } \\
\text { (days) }\end{array}$ & Compared to & $\begin{array}{c}\Delta \\
50 \% \\
\text { alive }\end{array}$ & $\begin{array}{l}p \text { value in } \\
50 \% \text { alive } \\
\text { (Fisher's } \\
\text { exact test) }\end{array}$ \\
\hline \multicolumn{7}{|c|}{ Figure 6} \\
\hline $\mathrm{N} 2$ & $(\mathrm{~A}),(\mathrm{B})$ & $>60$ & 20.39 & & & \\
\hline $\operatorname{aptf}-1(g k 794)$ & $\begin{array}{l}\text { (A) Batch 1, } \\
\text { (B) }\end{array}$ & $>60$ & 9.57 & & & \\
\hline rict-1 $(m g 360)$ & (A) Batch 1 & $>60$ & 25 & $\mathrm{~N} 2(\mathrm{~A})$ & $+22.6 \%$ & $\begin{array}{c}<0.001, * * * \\
\text { in day } 25\end{array}$ \\
\hline $\begin{array}{c}\text { rict-1 (mg360), aptf- } \\
1 \text { (gk794) }\end{array}$ & (A) Batch 1 & $>60$ & 12 & $\begin{array}{l}\text { aptf-1(gk794) } \\
\text { (A) Batch } 1\end{array}$ & $+25.4 \%$ & $\begin{array}{c}<0.001, * * * \\
\text { in day } 12\end{array}$ \\
\hline aptf-1 $(g k 794)$ & (A) Batch 2 & $>60$ & 9.54 & & & \\
\hline rict-1 $(m g 360)$ & (A) Batch 2 & & 17.93 & $\mathrm{~N} 2(\mathrm{~A})$ & $-16 \%$ & $\begin{array}{l}0.018, * \text { in } \\
\text { day } 17\end{array}$ \\
\hline
\end{tabular}




\begin{tabular}{|c|c|c|c|c|c|c|}
\hline $\begin{array}{c}\text { rict-1 (mg360), aptf- } \\
\text { l(gk794) }\end{array}$ & (A) Batch 2 & $>60$ & 11.18 & $\begin{array}{l}\text { aptf-1 }(g k 794) \\
\text { (A) Batch } 2\end{array}$ & $+17.2 \%$ & $\begin{array}{c}<0.001, * * * \\
\text { in day } 11\end{array}$ \\
\hline raga-1(ok386) & (B) & $>60$ & 18.56 & N2 (B) & $-8.97 \%$ & $\begin{array}{l}0.039, * \text { in } \\
\text { day } 19\end{array}$ \\
\hline $\begin{array}{c}\text { raga-1 }(\text { ok386) } \\
\text { aptf-1 }(g k 794)\end{array}$ & (B) & & 10.29 & $\begin{array}{c}a p t f-1(g k 794) \\
\text { (B) }\end{array}$ & $+7.5 \%$ & $\begin{array}{l}0.012, * \text { in } \\
\text { day } 10\end{array}$ \\
\hline \multicolumn{7}{|c|}{ Figure 7} \\
\hline $\mathrm{N} 2$ & (A), (B) & $>60$ & 20.39 & & & \\
\hline aptf-1 (gk794) & (A), (B) & $>60$ & 9.57 & & & \\
\hline cep-1(gk138) & (A) & $>60$ & 26.14 & N2 (A) & $+28.2 \%$ & $\begin{array}{l}<0.001, * * * \\
\text { in day } 25\end{array}$ \\
\hline $\begin{array}{c}\text { cep-1(gk138), aptf- } \\
1(g k 794)\end{array}$ & (A) & $>60$ & 9.88 & $\begin{array}{c}\operatorname{aptf-1}(g k 794) \\
\text { (A) }\end{array}$ & & $\begin{array}{l}\text { N.S. in day } \\
10\end{array}$ \\
\hline efk-1(ok3609) & (B) & $>60$ & 16.25 & N2 (B) & $-20.3 \%$ & $\begin{array}{c}<0.001, * * * \\
\text { in day } 16\end{array}$ \\
\hline $\begin{array}{c}\text { efk-1(ok3609), aptf- } \\
1 \text { (gk794) }\end{array}$ & (B) & $>60$ & 8.55 & $\begin{array}{c}\text { aptf-1 }(g k 794) \\
\text { (B) }\end{array}$ & $-10.7 \%$ & $\begin{array}{c}<0.001, * * * \\
\text { in day } 8\end{array}$ \\
\hline N2 & (C), (D) & $>60$ & 21.35 & & & \\
\hline aptf-1 $(g k 794)$ & (C), (D) & $>60$ & 8.88 & & & \\
\hline atg-18(gk378) & (C) & $>60$ & 4.48 & N2 (C) & $-79 \%$ & $\begin{array}{l}<0.001, * * * \\
\quad \text { in day } 4\end{array}$ \\
\hline $\begin{array}{c}\text { atg-18(gk378), aptf- } \\
1 \text { (gk794) }\end{array}$ & (C) & $>60$ & 4.49 & $\begin{array}{c}\operatorname{aptf-1}(g k 794) \\
\text { (C) }\end{array}$ & $-49.4 \%$ & $\begin{array}{l}<0.001, * * * \\
\quad \text { in day } 4\end{array}$ \\
\hline metr-1(ok521) & (D) & $>60$ & 16.78 & N2 (D) & $-21.4 \%$ & $\begin{array}{c}<0.001, * * * \\
\text { in day } 17\end{array}$ \\
\hline \multicolumn{7}{|c|}{ Figure 8} \\
\hline $\mathrm{N} 2$ & (A) & $>60$ & 21.35 & & & \\
\hline aptf-1 (gk794) & (A) Batch 1 & $>60$ & 8.88 & & & \\
\hline isp-1(qm150) & (A) Batch 1 & $>60$ & 23.67 & N2 (A) & $+10.9 \%$ & $\begin{array}{l}0.0028, * * \\
\text { in day } 23\end{array}$ \\
\hline $\begin{array}{c}\text { isp-1(qm150), aptf- } \\
1(g k 794)\end{array}$ & (A) Batch 1 & $>60$ & 14.31 & $\begin{array}{l}\text { aptf-1 }(g k 794) \\
\text { (A) Batch } 1\end{array}$ & $+61.1 \%$ & $\begin{array}{l}<0.001, * * * \\
\text { in day } 14\end{array}$ \\
\hline aptf-1 (gk794) & (A) Batch 2 & $>60$ & 9.54 & & & \\
\hline isp-1(qm150) & (A) Batch 2 & $>60$ & 21.95 & N2 (A) & & $\begin{array}{l}\text { N.S. in day } \\
23\end{array}$ \\
\hline $\begin{array}{c}\text { isp-1(qm150), aptf- } \\
1(g k 794)\end{array}$ & (A) Batch 2 & $>60$ & 16.36 & $\begin{array}{l}\text { aptf-1 }(g k 794) \\
\text { (A) Batch } 2\end{array}$ & $+71.5 \%$ & $\begin{array}{l}<0.001, * * * \\
\text { in day } 14\end{array}$ \\
\hline
\end{tabular}




\begin{tabular}{|c|c|c|c|c|c|c|}
\hline $\mathrm{N} 2$ & $(\mathrm{~B}),(\mathrm{C}),(\mathrm{D})$ & $>60$ & 26.9 & & & \\
\hline aptf-1(gk794) & $(\mathrm{B}),(\mathrm{C}),(\mathrm{D})$ & $>60$ & 10.05 & & & \\
\hline nuо-6(qm200) & (B) & $>60$ & 19.9 & N2 (B) & $-26 \%$ & $\begin{array}{l}<0.001, * * * \\
\text { in day } 21\end{array}$ \\
\hline $\begin{array}{c}\text { nuo-6(qm200), aptf- } \\
1(g k 794)\end{array}$ & (B) & $>60$ & 13.8 & $\begin{array}{c}a p t f-1(g k 794) \\
\text { (B) }\end{array}$ & $+37.3 \%$ & $\begin{array}{c}<0.001, * * * \\
\text { in day } 13\end{array}$ \\
\hline$m e v-1(k n 1)$ & (C) & $>60$ & 31.4 & $\mathrm{~N} 2(\mathrm{C})$ & $+16.7 \%$ & $\begin{array}{c}<0.001, * * * \\
\text { in day } 31\end{array}$ \\
\hline $\begin{array}{c}\text { mev-1(kn1), aptf- } \\
1(g k 794)\end{array}$ & (C) & $>60$ & 17.5 & $\begin{array}{c}a p t f-1(g k 794) \\
\text { (C) }\end{array}$ & $+74.1 \%$ & $\begin{array}{c}<0.001, * * * \\
\text { in day } 17\end{array}$ \\
\hline clk-1(qm30) & (D) & $>60$ & 8.38 & $\mathrm{~N} 2$ (D) & $-68.8 \%$ & $\begin{array}{c}<0.001, * * * \\
\text { in day } 10\end{array}$ \\
\hline $\begin{array}{c}\text { clk-1(qm30), aptf- } \\
1(g k 794)\end{array}$ & (D) & $>60$ & 6.47 & $\begin{array}{c}a p t f-1(g k 794) \\
\text { (D) }\end{array}$ & $-35.6 \%$ & $\begin{array}{l}<0.001, * * * \\
\text { in day } 10\end{array}$ \\
\hline \multicolumn{7}{|c|}{ Figure 9} \\
\hline $\mathrm{N} 2$ & Batch 1 & $>60$ & 23.54 & & & \\
\hline egl-4(ad450) & Batch 1 & $>60$ & 18.73 & N2 Batch 1 & $-20.4 \%$ & $\begin{array}{c}<0.001, * * * \\
\text { in day } 19\end{array}$ \\
\hline $\mathrm{N} 2$ & Batch 2 & $>60$ & 22.38 & & & \\
\hline egl-4(ad450) & Batch 2 & $>60$ & 17.68 & N2 Batch 2 & $-21 \%$ & $\begin{array}{c}<0.001, * * * \\
\text { in day } 17\end{array}$ \\
\hline egl-4(ad450) & Batch 3 & $>60$ & 18.51 & N2 Batch 2 & $-17.3 \%$ & $\begin{array}{c}0.001, * * \text { in } \\
\text { day } 19\end{array}$ \\
\hline$e g l-4(k s 61)$ & Batch 3 & $>60$ & 10.08 & N2 Batch 2 & $-54.9 \%$ & $\begin{array}{c}<0.001, * * * \\
\text { in day } 11\end{array}$ \\
\hline
\end{tabular}

Table S 5 Detail of survival spans of arrested L1 larvae with replicates are shown. Table is related to the figures 6-9. Displayed are the strains used, the related figure sub number with replicated batches, the scored animals number, the median survival, against which group was tested for $p$ value, the difference with the compared group and the $p$ value with the test day is estimated with the Fisher's test.

Table S 6

\begin{tabular}{|c|c|c|c|c|}
\hline Strain & $\mathrm{N}$ total & $\begin{array}{l}\text { Median of sleep } \\
\text { fraction }\end{array}$ & SE of mean & $\begin{array}{c}p \text { value } \\
\text { (Mann-Whitney U } \\
\text { test with Benjamini- } \\
\text { Hochberg Procedure } \\
\text { test) }\end{array}$ \\
\hline \multicolumn{5}{|c|}{ Figure S 2} \\
\hline $\mathrm{N} 2$ & 95 & $21.1 \%$ & $1.4 \%$ & \\
\hline daf-2(e1370) & 17 & $12.3 \%$ & $2.8 \%$ & \\
\hline $\begin{array}{c}d a f-2(e 1370), \text { aptf- } \\
1(g k 794)\end{array}$ & 16 & $1.6 \%$ & $0.4 \%$ & $* * *,<0.001$ \\
\hline daf-2(e979), aptf-1 (gk794) & 22 & $4 \%$ & $1.2 \%$ & $* * *,<0.001$ \\
\hline cep-1(gk138) & 23 & $22.1 \%$ & $1.9 \%$ & \\
\hline
\end{tabular}




\begin{tabular}{|c|c|c|c|c|}
\hline$i s p-1(q m 150)$ & 41 & $1.6 \%$ & $1.1 \%$ & $* * *,<0.001$ \\
\hline $\begin{array}{c}\text { isp-1(qm150), aptf- } \\
1(g k 794)\end{array}$ & 16 & $0.7 \%$ & $0.3 \%$ & $* * *,<0.001$ \\
\hline sir-2.2(tm2673) & 17 & $33.2 \%$ & $1.5 \%$ & $*, 0.011$ \\
\hline $\operatorname{sir}-2.2(\mathrm{tm} 2648)$ & 29 & $30.3 \%$ & $2 \%$ & $*, 0.017$ \\
\hline sir-2.4(n5137) & 21 & $19.3 \%$ & $1.8 \%$ & \\
\hline RIS(-) & 24 & $0 \%$ & $0.13 \%$ & $* * *,<0.001$ \\
\hline$c r h-1(t z 2)$ & 17 & $14.8 \%$ & $2.1 \%$ & \\
\hline fkh-9(ok1709) & 7 & $14.2 \%$ & $3.9 \%$ & \\
\hline zip-5(gk646) & 11 & $29.2 \%$ & $2.8 \%$ & \\
\hline$f k b-5(o k 653)$ & 5 & $17.9 \%$ & $5.4 \%$ & \\
\hline ags-3\&F32A6.2(gk517) & 10 & $14.3 \%$ & $3.2 \%$ & \\
\hline$n h r-129 \& n h r-168(g k 538)$ & 14 & $22 \%$ & $3.1 \%$ & \\
\hline ins-25(ok2773) & 4 & $7.5 \%$ & $3 \%$ & \\
\hline lec-3\&nlt-1(ok274) & 15 & $36.8 \%$ & $2 \%$ & $* * *,<0.001$ \\
\hline D1022.3(ok620) & 6 & $28.1 \%$ & $2.3 \%$ & \\
\hline gpa-10(pk362) & 15 & $13.8 \%$ & $1.3 \%$ & \\
\hline ife-4(ok320) & 8 & $27.7 \%$ & $4.8 \%$ & \\
\hline bus-1(e2678) & 11 & $20.2 \%$ & $2.3 \%$ & \\
\hline$d p y-19(e 1259)$ & 4 & $28.2 \%$ & $8.4 \%$ & \\
\hline C25E10.8(ok1753) & 6 & $23.5 \%$ & $2.3 \%$ & \\
\hline age-1 $(h \times 546)$ & 20 & $14.9 \%$ & $1.3 \%$ & \\
\hline phg-1(ok2741) & 41 & $39.6 \%$ & $1.6 \%$ & $* * *,<0.001$ \\
\hline$a a k-2(o k 524)$ & 11 & $16.4 \%$ & $1.3 \%$ & \\
\hline sma-5(n678) & 4 & $3.8 \%$ & $2.2 \%$ & \\
\hline nos-1(ok250) & 29 & $17.2 \%$ & $1.4 \%$ & \\
\hline osm-6(p811) & 8 & $10.1 \%$ & $2.1 \%$ & \\
\hline fasn-1 (g43) & 14 & $28.3 \%$ & $1.3 \%$ & \\
\hline $\operatorname{egl}-4(\operatorname{ad} 450)$ & 18 & $15.5 \%$ & $2.1 \%$ & \\
\hline$s b p-1(e p 79)$ & 27 & $19.7 \%$ & $1.5 \%$ & \\
\hline daf-7(e1372) & 13 & $23.2 \%$ & $2.2 \%$ & \\
\hline unc-31(e169) & 14 & $31 \%$ & $3.5 \%$ & \\
\hline akt-1 (mg306) & 30 & $22.8 \%$ & $1.2 \%$ & \\
\hline skn-1(zj15) & 14 & $11 \%$ & $2.2 \%$ & \\
\hline let-363(ok3018)/hT2 & 9 & $18.2 \%$ & $1.7 \%$ & \\
\hline smg-1(cc546) & 17 & $18.3 \%$ & $2.5 \%$ & \\
\hline par-4(it57) & 15 & $27.8 \%$ & $2.5 \%$ & \\
\hline \multicolumn{5}{|c|}{ Figure S 3} \\
\hline N2 & 46 & $19.7 \%$ & $1.1 \%$ & \\
\hline $\begin{array}{c}\text { Mock control daf- } \\
18(o k 480)\end{array}$ & 25 & $3.8 \%$ & $0.7 \%$ & \\
\hline pdaf-18::daf-18::myc & 48 & $20.5 \%$ & $1.5 \%$ & $* * *,<0.001$ \\
\hline pges-1::daf-18::myc & 52 & $12.9 \%$ & $1.1 \%$ & $* * *,<0.001$ \\
\hline pmyo-3::daf-18::myc & 65 & $7.7 \%$ & $0.5 \%$ & \\
\hline$p d p y-7:: d a f-18:: m y c$ & 23 & $11.6 \%$ & $1.5 \%$ & $* * *,<0.001$ \\
\hline
\end{tabular}

Table $\mathbf{S} 6$ Detail of mutantion screen for sleep alteration in arrested L1 larvae. Table is related to the figures S2 and S3. Displayed are the strains used, animals numbers, median of sleep fractions, SE of mean, the mutant groups were compared with the $\mathrm{N} 2$ control group. The $p$ value estimated with the MannWhitney $U$ and the Benjamini-Hochberg Procedure that for confirming the significance by multiple comparisons. 


\section{Appendix}

\subsection{C. elegans strains}

N2: wild type

HBR507: flp-11(tm2706) $X$.

HBR1021: goeIs 240 [hsp16.2::flp-11::SL2mKate2unc-54-3UTR, unc-119(+)].

HBR1403: aptf-1 (gk794) II, daf-2(e1370) III.

HBR1754: aptf-1(gk794) II, daf-16(mgDf50) I.

HBR1756: aptf-1(gk794) II, daf-2(e979) III.

HBR1777: goeIs384 [pflp-11::egl-1::SL2-mkate2-flp-11-3'utr, unc-119(+)]. (2bc)

HBR1809: aptf-1(gk794) II, zcIs4 [hsp-4::GFP] V.

HBR1811: aptf-1(gk794) II, gpIs1 [hsp-16.2p::GFP].

HBR1996: PF25B3.3::cyt_roGFP+myo-2::mCherry.

HBR1997: Punc-54::cyt_roGFP+myo-2::mCherry.

HBR1998: aptf-1(gk794) II, PF25B3.3::cyt_roGFP+myo-2::mCherry. (2bc)

HBR1999: aptf-1(gk794) II, Punc-54::cyt_roGFP+myo-2::mCherry. (2bc)

HBR2029: aptf-1 (gk794) II, aak-2 (ok524) X.

HBR2030: aptf-1 (gk794) II, aak-2 (ok524) X; aak-1(tm1944) III.

HBR2098: daf-18(ok480) IV. (2bc)

HBR2099: aptf-1(gk794) II, daf-18(ok480) IV.

HBR2100: sir-2.1(ok434) IV. (2bc)

HBR2101: aptf-1(gk794) II, sir-2.1(ok434) IV.

HBR2102: aptf-1(gk794) II, bnIs1 [pie-1::GFP::pgl-1 + unc-119(+)]. (2bc)

HBR2131: raga-1(ok386) II. (2bc)

HBR2132: aptf-1(gk794) II, raga-1(ok386) II.

HBR2133: efk-1(ok3609) III. (2bc)

HBR2134: aptf-1(gk794) II, efk-1(ok3609) III.

HBR2135: rict-1 (mg360) II. (2bc)

HBR2136: aptf-1 (gk794) II, rict-1(mg360) II.

HBR2137: isp-1(qm150) IV. (2bc)

HBR2138: aptf-1(gk794) II, isp-1(qm150) IV.

HBR2139: cep-1 (gk138) I. (2bc) 
HBR2140: aptf-1(gk794) II, cep-1(gk138) I.

HBR2199: aptf-1(gk794) II, atg-18(gk378) V.

HBR2218: $c l k-1$ (qm30) III. (2bc)

HBR2219: aptf-1 (gk794) II, clk-1(qm30) III. (2bc)

HBR2200: $\operatorname{atg}-18(g k 378) V$. (2bc)

HBR2220: $m e v-1(k n 1) I I I$. (2bc)

HBR2221: aptf-1(gk794) II, mev-1(kn1) III.

HBR2222: aptf-1(gk794) II, nuo-6(qm200) I. (2bc)

HBR2233: aptf-1(gk794) II, tut-1(tm 1297) IV. (2bc)

AGD397: aak-1(tm1944) III, aak-2(ok524) X, uthEx202 [crtc-1p::crtc-1

cDNA::tdTomato::unc-54 3'UTR + rol-6(su1006)].

BQ1: akt-1(mg306) $V$.

CB169: unc-31(e169) IV.

CB1259: $d p y$-19(e1259) III.

CB1370: daf-2(e1370) III.

CB1372: daf-7(e1372) III.

CB5609: bus-1(e2678) $\mathrm{V}$.

CE541: $s b p-1($ (ep 79) III.

CF1038: daf-16(mu86) I.

DA521: $\operatorname{egl-4}(\operatorname{ad} 450) I V$.

FK229: egl-4(ks61) IV.

FK312: $s m a-5(n 678) X$.

GG43: fasn-1 (g43) I.

GR1307: daf-16(mgDf50) $I$.

KK300: par-4(it57) $V$.

KX17: ife-4(ok320) $X$.

MIR13: sir-2.1(ok434) IV, aak-2(ok524) X.

MQ1333: nuо-6(qm200) I.

MT18068: sir-2.4(n5137) I,

NL1147: $g p a-10(p k 362) \mathrm{V}$.

PD8120: smg-1(cc546) $I$.

PR811: osm-6(p811) $\mathrm{V}$

QV225: $s k n-1(z j 15) I V$. 
RB518: nos-1(ok250) II.

RB754: aak-2(ok524) X.

RB808: D1022.3(ok620) II.

RB1428: lec-3\&nlt-1(ok274) II.

RB2075: phg-1(ok2741) II.

RB2098: ins-25(ok2773) I.

RG1087: daf-18(ok480) IV, veEx346 [Pges-1::daf-18::myc+str-1::gfp].

RG1083: daf-18(ok480) IV, veEx353 [Pmyo-3::daf-18::myc+str-1::gfp].

RG1170: daf-18(ok480) IV, veEx383 [Pdpy-7::daf-18::myc+str-1::gfp].

RG1174: daf-18(ok480) IV, veEx387 [str-1::gfp].

SJ4005: $z c I s 4$ [hsp-4::GFP] $V$.

SS747: bnIs1 [pie-1::GFP::pgl-1+unc-119(+)].

TJ1: cep-1(gk138) I.

TJ375: gpIs 1 [hsp-16-2p::GFP]. (2bc)

TJ1052: age-1(hx546) II.

TM2648: sir-2.2(tm2648) $X$.

TM2673: sir-2.2(tm2673) $X$.

VB1876: tut-1(tm1297) IV.

VC467: $f k b-5$ (ok653) I.

VC1124: $a g s-3 \& F 32 A 6.2(g k 517)$.

VC1172: $n h r-129 \& n h r-168(g k 538) \mathrm{V}$.

VC1277: C25E10.8(ok1753) $\mathrm{V}$.

VC1392: $z i p-5(g k 646) V$.

VC1925: fkh-9(ok1709) $X$.

VC2312: let-363(ok3018) I/hT2 [bli-4(e937) let-?(q782) qIs48] (I;III).

YT17: $c r h-1(t z 2)$ III.

YB409: daf-18(ok480) IV, tdEx239 [Pdaf-18::daf-18::myc+str-1::gfp].

The deletion alleles were backcrossed against wild-type N2. Backcross numbers are shown behind the genotype. 


\subsection{List of used primers}

aptf-1(gk794)

48 CGACAATCTTCCCAAAGACC

49 CGGATCGATTGCTAGAGAGG

50 GCTTGGACGGCTTTAGTTGA

daf-18(ok480)

1090 AGCATGGACTTCAACGATACAA

1091 GAATGCCAGATTGCCGAACA

1092 CGGTGGTCCATTTGAGATACC

aak-2(ok524)

893ATCGCCAAATTATGCTGCCC

894 GGCAGGGTTCCACAAAGAAG

895 AAGGAGACACTCGGAGTTGG

aak-1(tm1944)

865 AAGAAGATTTTCGGGCTTCT

866 ATCGTGGAAGAATGAAAGGA

867 TATGAAAACCAGGGCAGAAT

daf-16(mgDf50)

636 AGGTCAGAGTTCTAGGCGTAAA

637 CGGTTCCAGCAATTCCAAGT

638 AGTGGATTCTGAGCACACGA

sir-2.1(ok434)

1107 GTTTGATCACTCCTTCGCAAC

1108 TTCCCAGGACAGTTCGTACC

1109 GCGGAAATGGCAGAAGTAGT 
raga-1(ok386)

1195 ACCAGTCTTGTCTCGAAGCT

1196 CATCGCCAGGGATTGTTCG

1197 AAGGATCGGAACTGAAGCGA

efk-1(ok3609)

1205 CCTCGAATTCCATTTTCCTG

1206 GCCAAATTATGGGCTGAAGA

1207 CCAAACCCATTAGGAAACAGA

1208 GCAGCTCGTCTTACACCACA

rict-1(mg360)

1198 GATCTCAAATCCACCATCTGTGCGTC

1199 CGAGCCTGCTGGCAGTATCAAGTATAGA

1200 GAGCATGTACTTCCAGGTGTTGCACC

1201 TTCCTGATACTTCTCTGGGAGTTCAGGC

cep-1(gk138)

1192 CGTGACCAATACTACTGCACA

1193 AACGGGACATGGGACTTGAC

1194 GTTTCAATCGGAGGAACGCA

isp-1(qm150)

1188 TCAGGAGAAAAAAGACTGCATACCAATGGG

1189 AGTGTGCACCCATCTTGGATGTGGCT

1190 TCGTAGTGAGATCCGTGACAAGGGCAGTA

1191 CGTGGAAAGCCAGTCTTCGTCAAACATC

$\operatorname{atg-18}(\mathrm{gk378})$

1202 CGATAAACAGAGCGCAACGA

1203 GTTTGTTCGTCGGGGTATCC

1204 TGCCACCAAGCTAAATAGACTT 
mev-1(kn1)

1312 TTGACCTGGATGCTCTCAGG

1313 ACAACCGCTGATTCTATGGACTT

1314 CATTCCAACTGCGATTCTGTG

1315 AATCCTAAGAAGCGAATTCCGTT

пио-6(qm200)

1308 GAAGAGAGCGAAAACGGGTC

1309 GCACCGTATTCCTGTACGTAGA

1310 TTATTTGACGAGGATAACGACACA

1311 TCAGATATCGTGTCAAGGAGATTTT

clk-1(qm30)

1293 CCGACGATCCTGAAACACAC

1294 CGTGTCAGGACCGAAATCAA

1295 TAGACGCTGCTATTCCCCAG

daf-16(mu86)

468 AAAGAGCTCGTGGTGGGTTA

469 TCGCGCCTTTGTCTCTCTAT

470 AGTGTCGAGTGAAGGGAGC

daf-2(e1370)

519 CGGGATGAGACTGTCAAGATTGGAGATTTCGG

520 CAACACCTCATCATTACTCAAACCAATCCATG 


\section{Acknowledgments}

It was a wonderful experience in my life to do the Ph.D. at the Max-Planck-Institute for biophysical chemistry in Göttingen for three years and three months. This study would not have been possible without the support and help I received from many people.

First, I would like to give the warmest thanks to my supervisor Prof. Henrik Bringmann for all the professional guidance and advice he provided throughout my Ph.D. He was always open for discussion and was very supportive for solving any difficulties concerning my project. Next, I would like to sincerely thank my thesis committee members, Prof. Ralf Heinrich, and Prof. Nils Brose for their inspiration and the valuable comments on my project.

Many sincere thanks to Florentin Masurat and Jasmin Preis for the excellent teamwork during the publication process, and for the wonderful insights on my research and thesis. I would like to give special thanks to Inka Busack for proofreading my thesis and providing good ideas.

I would also like to extend many warm thanks to our group Royal Worm Force. I feel so lucky for having been a member of the team. Thank Michal Turek, Juliane Schwarz and Judith Besseling for helping me at the beginning of the Ph.D. Thank Elisabeth Maluck, Jan Konietzka, Anastasios Koutsoumparis, Yang Hu and Marina Sinner for giving every help and fun in work and life. Also, thank Sabine König and Silvia Gremmler for the great technical support.

I especially appreciate my family and all my friends who believed in me and supported me during the three years.

Last, I gratefully acknowledge the GGNB and the Systems Neuroscience program of the University Göttingen for all the opportunities and training. 
